\title{
Formation and evolution of star clusters in interacting galaxies
}

\author{
Dissertation \\ zur Erlangung des Doktorgrades der \\ Mathematisch-Naturwissenschaftlichen \\ Fakultäten \\ der Georg-August-Universität zu Göttingen
}

\author{
Vorgelegt von \\ Peter Anders \\ aus \\ Berlin
}

Göttingen 2006

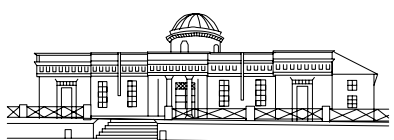


D 7

Referent: apl. Prof. Dr. U. Fritze - v. Alvensleben Korreferent: Dr. R. de Grijs

Tag der mündlichen Prüfung: 


\section{Contents}

1 Introduction \& Overview 1

1.1 Introduction . . . . . . . . . . . . . . . . 1

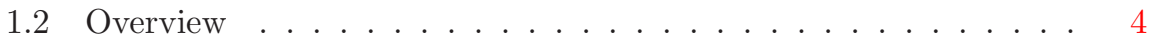

2 Evolutionary synthesis: Gaseous emission 9

2.1 Introduction . . . . . . . . . . . . . . . . . 9

2.2 Input Physics . . . . . . . . . . . . . . . . . . . . . . 10

2.2.1 SSP Models . . . . . . . . . . . . . . . . 10

2.2.2 Gaseous Emission . . . . . . . . . . . . . 10

2.2.3 The role of white dwarfs . . . . . . . . . . . . . 12

2.2.4 Filter Systems . . . . . . . . . . . . . . . . . 12

2.3 Time Evolution of SSP Spectra with Gaseous Emission . . . . . . 14

2.4 Impact of Gaseous Emission on Broad Band Luminosities and

Colors ...................... . . 14

2.4.1 Relative contributions of the gaseous emission - lines and continuum - to broad band fluxes . . . . . . . . 14

2.4.2 Time evolution of gaseous emission contributions . . . . . 16

2.4.3 Effects of gaseous emission on the color evolution of SSPs 17

2.5 Discussion . . . . . . . . . . . . . . . . 21

2.6 Structure and Description of Electronic Data-files . . . . . . . . . 22

2.7 Conclusions and Outlook . . . . . . . . . . . . . 22

2.8 Acknowledgements . . . . . . . . . . . . . . . 23

3 Systematic uncertainties in SED analysis 27

3.1 Introduction . . . . . . . . . . . . . . . . . . 27

3.2 Model description . . . . . . . . . . . . . . . . . . 28

3.2 .1 Input Models . . . . . . . . . . . . . . . . . 29

3.2.2 General description of the analysis algorithm . . . . . . . 29

3.2 .3 Artificial clusters . . . . . . . . . . . . . . . . . 32

3.3 Study of the accuracy of our analysis . . . . . . . . . . . . . . . 33

3.3.1 Passbands included in our analysis . . . . . . . . . . . 33

3.3.2 Choice of passband combination . . . . . . . . . . . 33

3.3.3 Varying the input parameters . . . . . . . . . . 37

3.3.4 Restricting the parameter space to the correct ranges . . 42

3.3.5 Restricting the parameter space to incorrect values . . . . 44

3.4 Conclusion . . . . . . . . . . . . . . . . . . 47

3.5 Acknowledgements . . . . . . . . . . . . . . . . . 49 
4 Star Cluster Formation in NGC 1569

4.1 Introduction . . . . . . . . . . . . . . . . . 53

4.2 Observations and data preparation . . . . . . . . . . . . . . 54

4.2.1 Source selection . . . . . . . . . . . . . 55

4.2.2 Photometric calibration . . . . . . . . . . . . . 56

4.2.3 Sample completeness . . . . . . . . . . . . . . 58

4.3 Parameters of the young clusters . . . . . . . . . . . . . . . . . 59

4.3.1 Cluster analysis algorithm . . . . . . . . . . . . 59

4.3.2 Parameter distributions . . . . . . . . . . . . . . 62

4.4 Cluster disruption vs. fading . . . . . . . . . . . . . . . . 69

4.5 The physical requirements for star cluster formation . . . . . . 73

4.6 Summary . . . . . . . . . . . . . . . . . . . . . . . . . . . . . . . 74

4.7 Acknowledgments . . . . . . . . . . . . . . 75

5 Photo press release on the NGC 1569 star cluster work $\quad 79$

6 Accurate photometry of extended sources 83

6.1 Introduction . . . . . . . . . . . . . . . . . . . 83

6.2 Determining accurate source sizes . . . . . . . . . . . . . 85

6.2.1 The parameters of the "standard" cluster . . . . . . . . . 86

6.2.2 Size determination as a function of input model . . . . . . 87

6.2.3 Effect of cluster brightness: Fits and fit errors . . . . . . . 95

6.2.4 Fitting radius variations . . . . . . . . . . . . . . . 95

6.2.5 Impact of the sky background . . . . . . . . . . . . . 100

6.2.6 Using the appropriate PSFs for fitting . . . . . . . . . . . 101

6.2.7 Other dependences . . . . . . . . . . . . . 102

6.2.8 Observing with ACS: chip, position, and filter dependence 104

6.2.9 Observing with NICMOS: filter-dependence . . . . . . . . 104

6.2.10 Further sources of uncertainties . . . . . . . . . . . 105

6.3 Determining accurate photometry: Aperture corrections . . . . . 112

6.3.1 Input parameters . . . . . . . . . . . . . . . . 112

6.3.2 The relation between aperture correction and input FWHM113

6.3.3 The relation between aperture correction and measured

FWHM ................... . . 113

6.3.4 Sky oversubtraction ................. . . 113

6.3.5 Filter dependence . . . . . . . . . . . . . 115

6.3.6 Subpixel shifts of clusters and the impact on the aperture

corrections . . . . . . . . . . . . . . 115

6.4 Cookbook for size-dependent aperture corrections . . . . . . . . . 117

6.5 Comparison of our method with the widely used DeltaMag method123

6.5.1 Size determination . . . . . . . . . . . . . . . . 123

6.5.2 Aperture corrections . . . . . . . . . . . . . 126

6.6 Summary . . . . . . . . . . . . . . . . . . . 127

6.7 Acknowledgements . . . . . . . . . . . . . . . . . . . 128

6.8 Appendices . . . . . . . . . . . . . . . . . . . 131

6.8.1 Parameters of cluster sizes fits . . . . . . . . . . . 131

6.8.2 Parameters of aperture correction fits: Intrinsic sizes . . . 145

6.8.3 Parameters of aperture correction fits: Measured sizes . . 173

6.8.4 Illustrative figures . . . . . . . . . . . . . . . . . 201 
7 Young star clusters in the Antennae galaxies 205

7.1 Introduction . . . . . . . . . . . . . . . 205

7.2 Cluster luminosity and mass functions . . . . . . . . . . . 206

7.3 Observational data and analysis method . . . . . . . . . . 207

7.3.1 Source selection and photometry . . . . . . . . . . 207

7.3.2 Cluster completeness . . . . . . . . . . . . . . 207

7.3.3 Statistical turnover determination . . . . . . . . . . 208

7.4 The cluster luminosity function in the Antennae system . . . . . 208

7.5 Summary and conclusions . . . . . . . . . . . . . . . . . 209

7.6 Acknowledgments . . . . . . . . . . . . . . . . . . . 210

8 Further publications

8.1 The photometric evolution of dissolving star clusters I: First pre-

dictions . . . . . . . . . . . . . . . . . . 213

8.2 How well do we know the age and mass distributions of the star cluster system in the Large Magellanic Cloud? . . . . . . . . . . 214

8.3 Systematic uncertainties in the analysis of star cluster parameters based on broad-band imaging observations . . . . . . . . . . . . . 215

8.4 CIRPASS near-infrared integral-field spectroscopy of massive star clusters in the starburst galaxy NGC 1140 . . . . . . . . . . . 215

8.5 Star Cluster Formation and Evolution in

Nearby Starburst Galaxies: II. Initial Conditions . . . . . . . . . 216

8.6 Star Cluster Formation and Evolution in

Nearby Starburst Galaxies: I. Systematic Uncertainties . . . . . 217

8.7 Stellar Populations and Star Cluster Formation in Interacting Galaxies with the Advanced Camera for Surveys . . . . . . . . 218

8.8 Conference proceedings . . . . . . . . . . . . 218

9 Summary \& Outlook 221

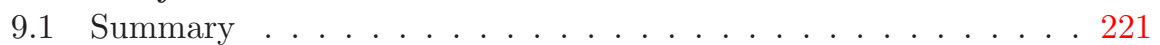

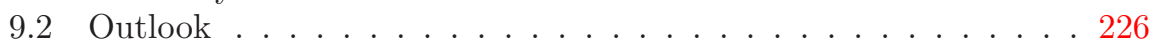

10 Acknowledgements $\quad 229$

11 Curriculum Vitae $\quad 231$ 


\section{List of Figures}

2.1 Spectrum in terms of flux $F_{\lambda}$ as a function of wavelength $\lambda$ at 3 different times for an SSP of solar metallicity (a) and metallicity $Z=0.0004$ (b), both with Salpeter IMF. . . . . . . . . . . . 15

2.2 Spectrum in terms of flux $F_{\lambda}$ as a function of wavelength $\lambda$ for an SSP with $Z=0.0004$ and Salpeter IMF at an age of 4 Myr, with and without gaseous emission, in log-log presentation for better visibility. . . . . . . . . . . . . . . . 16

2.3 Relative contributions of the gaseous emission - lines and continuum - to broad band fluxes $U$. . . $K$ at solar metallicity (upper panel) and low metallicity $Z=0.0004$ (lower panel).

2.4 Decomposition of the total gaseous emission in terms of lines and continuum contributions in various filters bands $U$. . $K$ at an age of $4 \mathrm{Myr}$ at solar metallicity (upper panel) and low metallicity $Z=0.0004$ (lower panel). . . . . . . . . . .

2.5 Time evolution of the gaseous emission contribution to broad band fluxes $U, B, V, I$, and $K$ at solar metallicity (a) and low metallicity $Z=0.0004$ (b)

2.6 Time evolution of the Johnson colors $V-I$ with and without gaseous emission at solar metallicity $Z=0.02$ and low metallicity

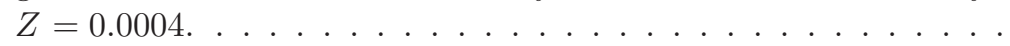

2.7 Time evolution of the magnitude in the Johnson $V$-band with and without gaseous emission at solar metallicity $Z=0.02$ and low metallicity $Z=0.0004$ for SSPs of the same mass. . . . . . .

2.8 Comparison of our models with STARBURST99 models with different mass-loss rates, for solar metallicity and the Johnson color $B-V \ldots \ldots \ldots \ldots \ldots \ldots \ldots$

3.1 Representative SEDs, for the 5 available metallicities and for 5 different, representative ages. The extinction is set to zero, and a Salpeter IMF is used. We plot the absolute magnitudes in the respective Hubble Space Telescope (HST) passbands for $M_{\text {cluster }}=M_{\text {model }}$ as a function of the effective wavelengths of the HST passbands (see section 3.3.1); the labels on the horizontal axis are the corresponding standard Johnson passbands. . . .

3.2 Dispersion of recovered properties of artificial clusters, assuming availability of UBVRIJH and passband combinations rejecting one of the UBVRIJH passbands, as indicated in the legend. Cluster parameters are standard. . . . . . . . . . . . 34 
3.3 Dispersion of recovered properties of artificial clusters, assuming availability of various optical passband combinations, as indicated in the legend. Cluster parameters are standard. . . . . . . . . . 35

3.4 Dispersion of recovered properties of artificial clusters, assuming availability of various optical+NIR passband combinations, as indicated in the legend. Cluster parameters are standard. . . . . 35

3.5 Dispersion of recovered properties of artificial clusters, comparing various wide and medium-band HST filters, as indicated in the legend. Cluster parameters are standard. . . . . . . . . . 36

3.6 Dispersion of recovered properties of artificial clusters, assuming availability of UBVRIJH magnitudes and varying observational errors, as indicated in the legend. Other parameters are standard. 38

3.7 Dispersion of recovered properties of artificial clusters, assuming availability of UBVRIJH magnitudes and varying internal extinction values, as indicated in the legend. Other parameters are standard. . . . . . . . . . . . . . . 3 38

3.8 Dispersion of recovered properties of artificial clusters, assuming availability of UBVRIJH magnitudes and varying metallicity values, as indicated in the legend. Other parameters are standard. 39

3.9 Dispersion of recovered properties of artificial clusters, assuming availability of $U B I H$ magnitudes and varying observational errors, as indicated in the legend. Other parameters are standard. . . . 40

3.10 Dispersion of recovered properties of artificial clusters, assuming availability of $U B I H$ magnitudes and varying internal extinction values, as indicated in the legend. Other parameters are standard. 40

3.11 Dispersion of recovered properties of artificial clusters, assuming availability of $U B I H$ magnitudes and varying metallicity values, as indicated in the legend. Other parameters are standard. . . .

3.12 Dispersion of recovered properties of artificial clusters, assuming availability of UBVRIJH and passband combinations without one of the UBVRIJH passbands, as indicated in the legend. Solutions were sought with metallicity fixed to the input value. Cluster parameters are standard. . . . . . . . . . . . .

3.13 Dispersion of recovered properties of artificial clusters, assuming availability of UBVRIJH and passband combinations without one of the UBVRIJH passbands, as indicated in the legend. Solutions were sought with extinction fixed to the input value. Cluster parameters are standard. . . . . . . . . . . . .

3.14 Dispersion of recovered properties of artificial clusters, assuming availability of UBVRIJH and passband combinations without one of the UBVRIJH passbands, as indicated in the legend. Solutions were sought with extinction and metallicity fixed to the input values. Cluster parameters are standard. . . . . . . . . . . .

3.15 Dispersion of recovered properties of artificial clusters, assuming availability of UBVRIJH and various input metallicities, as indicated in the legend. Solutions were sought with metallicity fixed to solar metallicity. Cluster parameters are standard. . . . . . . . 
3.16 Dispersion of recovered properties of artificial clusters, assuming availability of $U B I H$ and various input metallicities, as indicated in the legend. Solutions were sought with metallicity fixed to solar metallicity. Cluster parameters are standard. . . . . . . . . 45

3.17 Dispersion of recovered properties of artificial clusters, assuming availability of UBVRIJH and various input extinction values, as indicated in the legend. Solutions were sought with extinction fixed to $E(B-V)=0.1$. Cluster parameters are standard. . . . . . . 46

3.18 Dispersion of recovered properties of artificial clusters, assuming availability of $U B I H$ and various input extinction values, as indicated in the legend. Solutions were sought with extinction fixed to $E(B-V)=0.1$. Cluster parameters are standard. . . . . . . . . .

4.1 Distribution of the Gaussian $\sigma$ 's of the detected sources. Two Gaussians were fitted to this distribution, representing stars and cluster candidates, respectively. . . . . . . . . . . 56

4.2 Image of the inner part of NGC 1569 (our small FoV) with the positions of the clusters marked. Some clusters (and one apparent star) are labelled. North is marked by the arrow, east is indicated by the line perpendicular to the arrow. . . . . . . . . . 58

4.3 Comparison of the cluster photometry obtained from H00 and in this work. Displayed is the dependence of the magnitude difference on the difference of the source apertures used. Naming of 4 individual clusters is following H00. Lines are included to guide the eye. . . . . . . . . . . . . . . .

4.4 Completeness curves for NGC 1569. The different line styles refer to different passbands, as indicated in the legend. These completeness curves apply to the small FoV. The horizontal lines indicate 0, 50, 90 and 100 per cent completeness. Magnitudes are given in STMAG, the standard HST zero-point system, based on a flat reference spectrum. . . . . . . . . . . . . . 60

4.5 Parameter distributions of the clusters in the small FoV; open histograms: all ages, shaded histograms: only ages > 8 Myr. . . .

4.6 Model SEDs for $\mathrm{Z}=0.004$ and 5 different young ages. SEDs are shifted to coincide at the $H$ band. . . . . . . . . . . . .

4.7 Mass functions of NGC 1569 cluster candidates in three age bins (shaded histograms; ages as indicated in each panel) and the total mass function (open histograms). Vertical lines indicate completeness limits (see text for details). . . . . . . . . .

4.8 Mass functions of NGC 1569 cluster candidates in two age bins (as indicated), with age uncertainties entirely in this age bin. Left panels: best values. Right panels: Extreme cases allowed by uncertainty estimates (young ages: upper limits; older ages: lower limits). See text for details. . . . . . . . . . . . . . .

4.9 Parameter distributions of the two FoVs: open histograms = small FoV (including NICMOS coverage), shaded histograms = large FoV (without NICMOS coverage). . . . . . . . . . . . 
4.10 Comparison of parameters for certain metallicity restrictions (shaded histograms) for the small FoV. Upper panel: $\log ($ age) distributions, lower panel: $\log$ (mass) distributions. The allowed metallicity ranges are shown in the panels $(\mathrm{Z} 1 \equiv[\mathrm{Fe} / \mathrm{H}]=-1.7, \mathrm{Z} 2 \equiv$ $[\mathrm{Fe} / \mathrm{H}]=-0.7, \mathrm{Z} 3 \equiv[\mathrm{Fe} / \mathrm{H}]=-0.4, \mathrm{Z} 4 \equiv[\mathrm{Fe} / \mathrm{H}]=0, \mathrm{Z} 5 \equiv[\mathrm{Fe} / \mathrm{H}]=+0.4)$. The results for no metallicity restriction are shown as open histograms, for comparison. . . . . . . . . . . . . 70

4.11 Comparison of parameters, derived with all metallicities allowed (open histograms) and with metallicity fixed to solar (shaded histograms) . . . . . . . . . . . . . . . 71

4.12 Cluster age distribution, affected by fading and cluster disruption. Linear relations are indicated, and slopes given in the legend; see text for details. . . . . . . . . . . . . . . . 72

4.13 Cluster mass distribution for 3 different age bins, as indicated in the legends. Best-fitting slopes (and uncertainties) shown, as described in Section 4.4. . . . . . . . . . . . 72

5.1 Photo press release on the star clusters in NGC 1569 . . . . . . . 81

6.1 Fitted Gaussian FWHMs for input Gaussian profile convolved with WFPC2/WF3 F555W PSF, located on the central pixel. The diagonal solid line in the top panel represents a one-to-one relation. Top: Output FWHM. Bottom: Output - input FWHM. 89

6.2 Model light distributions. . . . . . . . . . . . . . . . . . 90

6.3 Fitted Gaussian FWHM for input King profiles with different concentrations: $*, c=5 ; \mathbf{\square}, c=30 ; \odot, c=100$. The diagonal solid line represents a one-to-one relation. . . . . . . . . . . 92

6.4 Fitted Gaussian FWHM for input EFF profiles with different power-law indices: $*, \gamma=1.5 ; \mathbf{\square}, \gamma=2.5$. The diagnonal solid line represents a one-to-one relation. . . . . . . . . . . . 93

6.5 Fitting standard artificial clusters with different input profiles, using the input profile shape as fitting profiles (instead of the Gaussian). The diagnonal solid line represents a one-to-one relation. Top: Different King profiles. Bottom: Different EFF profiles. 94

6.6 Fitted Gaussian FWHM for input King 30 profiles of different cluster magnitudes. Top: Output - input sizes, simulated data and fitted polynomials. Bottom: Comparison of fit functions, using the $V=10 \mathrm{mag}$ fit function as reference. . . . . . . . . . 96

6.7 Fitted Gaussian FWHM for input EFF 15 profiles of different cluster magnitudes. Top: Output - input sizes, simulated data and fitted polynomials. Bottom: Comparison of fit functions, using the $V=10 \mathrm{mag}$ fit function as reference. . . . . . . . . . 97

6.8 Comparison for $V=10$ mag King 30 (upper panel) and EFF 15 (lower panel) clusters. Horizontal lines indicate \pm 0.2 pixels and \pm 0.5 pixels. The solid curved lines indicate the $\pm 1 \sigma$ range of the scatter. Shown are the differences of the individual runs with respect to the average value. . . . . . . . . . . . . . . 98 
6.9 Comparison for $V=14$ mag King 30 (upper panel) and EFF 15 (lower panel) clusters. Horizontal lines indicate \pm 0.5 and \pm 1.0 pixels. The solid curved lines indicate the $\pm 1 \sigma$ range of the scatter. Shown are the differences of the individual runs with respect to the average value. . . . . . . . . . . .

6.10 Conversion relations for a standard cluster, using different fitting radii (given in pixel units in the legend). . . . . . . . . . . . . . . 101

6.11 Conversion relations for a standard cluster, using different fitting radii (given in pixel units in the legend). Displayed are the offsets of these relations from the relation for a fitting radius of 5 pixels. 102

6.12 Conversion relations for a standard cluster, using different fitting radii (given in pixel units in the legend) and taking the input light profile, PSF and diffusion kernel into account. . . . . . . . . 103

6.13 Conversion relations for a $V=10 \mathrm{mag}$ cluster, taking sky noise into account. As reference, the data for a standard cluster are taken. The straight lines are at \pm 0.2 pixel. . . . . . . . . . . 105

6.14 Conversion relations for a $V=14$ mag cluster, taking sky noise into account. As reference, the data for a standard cluster are taken. The straight lines are at \pm 0.5 pixel. . . . . . . . . . . 106

6.15 Scatter for $V=10$ mag EFF 15 clusters, with varying sky levels ("BG", in ADU). As reference, the average data are taken. The straight lines are at \pm 0.2 and \pm 0.5 pixels. The solid curved lines indicate the $\pm 1 \sigma$ range of the scatter. . . . . . . . . . . 107

6.16 Scatter for $V=14$ mag EFF 15 clusters, with varying sky level ("BG", in ADU). As reference, the average data are taken. The straight lines are at \pm 0.5 and \pm 1.0 pixels. The solid curved lines indicate the $\pm 1 \sigma$ range of the scatter. . . . . . . . . . . 108

6.17 Conversion relations for a standard cluster, using the appropriate PSFs for the fitting. The horizontal lines indicate \pm 0.2 pixel. . . 109

6.18 Conversion relations for a standard cluster, using different filters for the ACS/WFC (chip 1). . . . . . . . . . . . . . . 110

6.19 Conversion relations for a standard cluster (Upper panel: King 30 profile; Lower panel: EFF 15 profile), using different filters for NICMOS camera 2. . . . . . . . . . . . . . . . 111

6.20 Upper panel: Theoretical AC values (to infinite radius) for EFF 15 profiles with different FWHMs, and best fit results. The aperture sizes used are given. Lower panel: Deviations of data from fits. . . . . . . . . . . . . . . . . . . 114

6.21 Theoretical AC values for EFF 15 profiles with different FWHMs, and best fit results. . . . . . . . . . . . . . . . 115

6.22 Upper panel: Theoretical AC values (to infinite radius) for EFF 15 profiles with different measured FWHMs, and best fit results. The aperture sizes used are given. Lower panel: Deviations of data from fits, covering the whole range of fit accuracies. . . . . . 116

6.23 Correction for sky oversubtraction as a function of input FWHM, following Eq. (6.8), for a 3-pixel source annulus, 5/8-pixel inner/outer sky annulus, and an EFF 15 profile. . . . . . . . . 117

6.24 Filter dependence of the $\mathrm{AC}$ for a 3-pixel aperture, in $U B V I$ for the WF3 (upper panel) and the PC (lower panel) chip of the WFPC2, and assuming an EFF 15 profile. . . . . . . . . . . . 118 
6.25 ACs for clusters with subpixel shifts relative to the $[0 ; 0]$ point, assuming a standard cluster with an EFF 15 profile. Shown are the absolute deviations between differently centered clusters with respect to the fit for the cluster at $[0 ; 0]$. Top panel: AC 3 pixels $\rightarrow \infty$. Middle panel: AC 5 pixels $\rightarrow \infty$. Bottom panel: AC 8 pixels $\rightarrow \infty \ldots \ldots \ldots \ldots \ldots$. . . . . . . . . . . . . . . . . .

6.26 ACs for clusters with subpixel shifts relative to the $[0 ; 0]$ point, assuming a standard cluster with an EFF 15 profile. Shown are the relative deviations between differently centered clusters with respect to the fit for the cluster at $[0 ; 0]$. Top panel: AC 3 pixels $\rightarrow \infty$. Middle panel: AC 5 pixels $\rightarrow \infty$. Bottom panel: AC 8 pixels $\rightarrow \infty$. . . . . . . . . . . . . . . . . . . 120

6.27 ACs for clusters with annuli shifts relative to the $[0 ; 0]$ point, assuming a standard cluster with an EFF 15 profile. Shown are the absolute deviations between differently centered annuli with respect to the fit for the cluster at [0;0]. Top panel: AC 3 pixels $\rightarrow \infty$. Middle panel: AC 5 pixels $\rightarrow \infty$. Bottom panel: AC 8

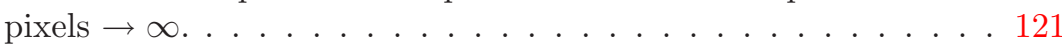

6.28 ACs for clusters with annuli shifts relative to the $[0 ; 0]$ point, assuming a standard cluster with an EFF 15 profile. Shown are the relative deviations between differently centered annuli with respect to the fit for the cluster at $[0 ; 0]$. Top panel: AC 3 pixels $\rightarrow \infty$. Middle panel: AC 5 pixels $\rightarrow \infty$. Bottom panel: AC 8 pixels $\rightarrow \infty$. . . . . . . . . . . . . . . . . . . . . . . . . . 122

6.29 Top panel: $\Delta$ mag between 0.5 and 3 pixels, for 3 different profiles. Middle panel: Comparison of $\Delta$ mag for off-centered aperture annuli for the EFF 15 profile. Bottom panel: Comparison of $\Delta$ mag for different centerings of clusters on a pixel, using an EFF 15 profile. . . . . . . . . . . . . . . . . . 124

6.30 Scatter in the size determination from the DeltaMag method (assuming a photometric accuracy of $\pm 0.1 \mathrm{mag}$ ), compared to the scatter introduced by our method for a standard cluster. Top panel: King 30 profile. Bottom panel: EFF 15 profile. . . . . . . 125

6.31 Scatter in the size determination from the DeltaMag method (assuming a photometric accuracy of $\pm 0.1 \mathrm{mag}$ ), compared to the scatter introduced by our method for a $V=14$ mag cluster. Top panel: King 30 profile. Bottom panel: EFF 15 profile. . . . . . . 126

6.32 Scatter in the AC calculation from the DeltaMag method (assuming a photometric accuracy of $\pm 0.1 \mathrm{mag}$ ), compared to the scatter introduced by our method for a standard cluster. Top panels: 3 pixel radius apertures for an EFF 15 profile (left) and a King 30 profile (right). Bottom panels: Assuming an EFF 15 profile, and using 5 pixel (left) and 8 pixel (right) radius apertures. 127

6.33 Various model light distributions. Left: Double-linear display. Right: Double-log display. . . . . . . . . . . . . . . . . . 201

6.34 Light profile residuals of King 30 clusters. Rows (from top to bottom): $\mathrm{FWHM}=0.5,5.0$ and 10.0 pixels. Columns (from left to right): fitting radius $=5,9,15$ pixels. Color scale is linear, with very dark/bright regions having the largest deviations. Gray-scales are identical within a row. . . . . . . . . . . 202 
6.35 Light profile residuals of EFF 15 clusters. Rows (from top to bottom): $\mathrm{FWHM}=0.5,5.0$ and 10.0 pixels. Columns (from left to right): fitting radius $=5,9,15$ pixels. Color scale is linear, with very dark/bright regions having the largest deviations. Gray-scales are identical within a row. . . . . . . . . . . . . 202

6.36 PSFs for the WF3 chip and the F555W filter, plotted using logarithmic color coding. The position in the image corresponds to the respective position on the chip. The PSFs were created subsampled by a factor of 10 , the displays show $200 \times 130$ pixels per PSF, corresponding to $2 \times 1.3$ arcsec for an observation. . . . . . 203

7.1 Comparing the different model fits with the observations: The best fits of the Gaussian (left panel) and the power-law (right panel) models for the absolute $V$-band LF, in arbitrary units. The grey line is a kernel density estimate of the observed data and the black line shows the Maximum Likelihood fit multiplied by the completeness function. The dashed line corresponds to the estimated LF without taking into account the varying completeness fractions (scaled arbitrarily w.r.t. the observations). . . 209 


\section{List of Tables}

2.1 Non-hydrogen emission lines and their line strengths, normalized to $\mathrm{H}_{\beta}$ line strength, as a function of metallicity $(Z 1=0.0004$, $\left.\mathrm{Z} 2=0.004, \mathrm{Z} 3=0.008, \mathrm{Z} 4=0.02=Z_{\odot}, Z 5=0.05\right) . . .$.

3.1 Overview of the most important filters and most/least preferable 4-passband combinations, if NIR data are available . . . . . . . 48

3.2 Overview of the most important filters and most/least preferable 4-passband combinations, if no NIR data are available . . . . . . 49

4.1 Overview of the observations of NGC 1569 . . . . . . . . . . 54

4.2 Comparison of parameters of clusters with literature values . . . 67

6.1 Conversion factors to calculate a model's $R_{\text {eff }}=R_{1 / 2}$ from its FWHM. . . . . . . . . . . . . . . . 88

6.2 Count rates [in ADU] in the peak pixel of selected clusters, to approximate S/N ratios for the background levels . . . . . . . . . 104

6.3 The sizes of the PSFs as such, measured using the same procedure as for the clusters. "Pre" and "Post" refer to either before or after the installation of the NICMOS cryocooler in the year 2002. . . . 131

6.4 Fit results of cluster sizes for a "standard" cluster. Upper panel: $\operatorname{Size}(x)$; Lower panel: $\operatorname{Size}^{\prime}(y) \ldots$. . . . . . . . . . . . . . . 132

6.5 Fit results of cluster sizes for a "standard" cluster, using various filters: $U=\mathrm{F} 336 \mathrm{~W}, B=\mathrm{F} 439 \mathrm{~W}, \mathrm{H} \alpha=\mathrm{F} 656 \mathrm{~N}, I=\mathrm{F} 814 \mathrm{~W}$. See

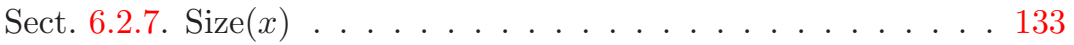

6.6 Fit results of cluster sizes for a "standard" cluster, using various filters: $U=\mathrm{F} 336 \mathrm{~W}, B=\mathrm{F} 439 \mathrm{~W}, \mathrm{H} \alpha=\mathrm{F} 656 \mathrm{~N}, I=\mathrm{F} 814 \mathrm{~W}$. See

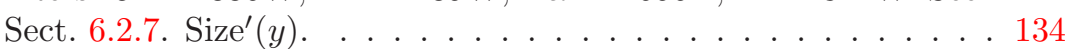

6.7 Fit results of cluster sizes for a "standard" cluster, using various WFPC2 chips. See Section 6.2.7. Size $(x)$. . . . . . . . . . . 135

6.8 Fit results of cluster sizes for a "standard" cluster, using various WFPC2 chips. See Section 6.2.7. Size'(y). . . . . . . . . . . 136

6.9 Fit results of cluster sizes for a "standard" cluster, using various filters, and using the appropriate PSF for the fitting: $U=$ F336W, $B=\mathrm{F} 439 \mathrm{~W}, V=\mathrm{F} 555 \mathrm{~W}, I=\mathrm{F} 814 \mathrm{~W}$. See Section 6.2.6. $\operatorname{Size}(x) \ldots \ldots \ldots \ldots \ldots \ldots \ldots$

6.10 Fit results of cluster sizes for a "standard" cluster, using various filters, and using the appropriate PSF for the fitting: $U=$ F336W, $B=\mathrm{F} 439 \mathrm{~W}, V=\mathrm{F} 555 \mathrm{~W}, I=\mathrm{F} 814 \mathrm{~W}$. See Section 6.2.6. $\operatorname{Size}^{\prime}(y) \ldots \ldots \ldots \ldots$. . . . . . . . . . . . . . 138 
6.11 Fit results of cluster sizes for a "standard" cluster, using various filters available for the ACS/WFC (chip 1, except V2): $B$ $=\mathrm{F} 435 \mathrm{~W}, V=\mathrm{F} 555 \mathrm{~W}, V 2=\mathrm{F} 555 \mathrm{~W}$ chip2, $I=\mathrm{F} 814 \mathrm{~W}$. See

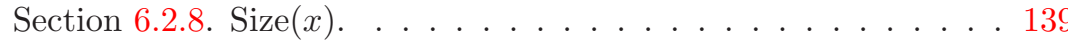

6.12 Fit results of cluster sizes for a "standard" cluster, using various filters available for the ACS/WFC (chip 1, except V2): $B$ $=\mathrm{F} 435 \mathrm{~W}, V=\mathrm{F} 555 \mathrm{~W}, V 2=\mathrm{F} 555 \mathrm{~W}$ chip2, $I=\mathrm{F} 814 \mathrm{~W}$. See

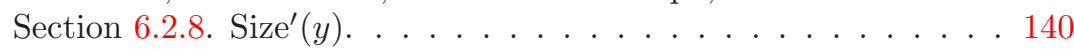

6.13 Fit results of cluster sizes for a "standard" cluster, using various filters available for the NICMOS camera NIC2 $(J=\mathrm{F} 110 \mathrm{~W}, H$ $=\mathrm{F} 160 \mathrm{~W}, K=\mathrm{F} 205 \mathrm{~W})$ and 2 different epochs of observation ("1st Feb 1998" shows severe blurring due to focus shifts in the pre-cooler phase, which is not present for all pre-service mission observations; "late 2002" is an in-focus observation in the postcooler phase.). See Section 6.2.9. Size $(x)$

6.14 Fit results of cluster sizes for a "standard" cluster, using various filters available for the NICMOS camera NIC2 $(J=\mathrm{F} 110 \mathrm{~W}, H$ $=\mathrm{F} 160 \mathrm{~W}, K=\mathrm{F} 205 \mathrm{~W})$ and 2 different epochs of observation ("1st Feb 1998" shows severe blurring due to focus shifts in the pre-cooler phase, which is not present for all pre-service mission observations; "late 2002" is an in-focus observation in the postcooler phase.). See Section 6.2.9. Size $(x)$. . . . . . . . . . . .

6.15 Fit results of cluster sizes for a "standard" cluster, using various filters available for the NICMOS camera NIC2 $(J=\mathrm{F} 110 \mathrm{~W}, H=\mathrm{F} 160 \mathrm{~W}$, $K=\mathrm{F} 205 \mathrm{~W}$ ) and 2 different epochs of observation ("1st Feb 1998" shows severe blurring due to focus shifts in the pre-cooler phase, which is not present for all pre-service mission observations; "late 2002" is an in-focus observation in the post-cooler phase.). See Section 6.2.9.

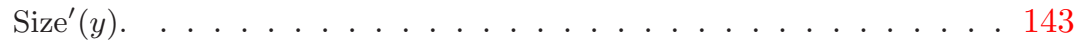

6.16 Fit results of cluster sizes for a "standard" cluster, using various filters available for the NICMOS camera NIC2 $(J=\mathrm{F} 110 \mathrm{~W}, H=\mathrm{F} 160 \mathrm{~W}$, $K=\mathrm{F} 205 \mathrm{~W}$ ) and 2 different epochs of observation ("1st Feb 1998" shows severe blurring due to focus shifts in the pre-cooler phase, which is not present for all pre-service mission observations; "late 2002" is an in-focus observation in the post-cooler phase.). See Section 6.2.9. $\operatorname{Size}^{\prime}(y) \ldots \ldots \ldots$. . . . . . . . . . . . . . 144

6.17 Fit result of Eq. (6.7) to different apertures and models, for the correction to infinite aperture, as a function of the intrinsic size of a cluster. PC chip, F555W filter . . . . . . . . . . . . . 146

6.18 Fit result of Eq. (6.7) to different apertures and models, for the correction to infinite aperture, as a function of the intrinsic size of a cluster. PC chip, F555W filter . . . . . . . . . . . . 147

6.19 Fit result of Eq. (6.7) to different apertures and models, for the correction to infinite aperture, as a function of the intrinsic size of a cluster. PC chip, F555W filter . . . . . . . . . . . . . . . 148

6.20 Fit result of Eq. (6.7) to different apertures and models, for the correction to infinite aperture, as a function of the intrinsic size of a cluster. WF3 chip, F555W filter . . . . . . . . . . . . . . 149 
6.21 Fit result of Eq. (6.7) to different apertures and models, for the correction to infinite aperture, as a function of the intrinsic size of a cluster. WF3 chip, F555W filter . . . . . . . . . . . . 150

6.22 Fit result of Eq. (6.7) to different apertures and models, for the correction to infinite aperture, as a function of the intrinsic size of a cluster. WF3 chip, F555W filter . . . . . . . . . . . . . . 151

6.23 Fit result of Eq. (6.7) to different apertures and models, for the correction to infinite aperture, as a function of the intrinsic size of a cluster. ACS/WFC1 chip, F555W filter (chip 2 is equivalent) 152

6.24 Fit result of Eq. (6.7) to different apertures and models, for the correction to infinite aperture, as a function of the intrinsic size of a cluster. ACS/WFC1 chip, F555W filter (chip 2 is equivalent) 153

6.25 Fit result of Eq. (6.7) to different apertures and models, for the correction to infinite aperture, as a function of the intrinsic size of a cluster. ACS/WFC1 chip, F555W filter (chip 2 is equivalent) 154

6.26 Fit result of Eq. (6.7) to different apertures and models, for the correction to infinite aperture, as a function of the intrinsic size of a cluster. NICMOS/NIC2 chip, F110W filter, pre-cooler . . . . 155

6.27 Fit result of Eq. (6.7) to different apertures and models, for the correction to infinite aperture, as a function of the intrinsic size of a cluster. NICMOS/NIC2 chip, F110W filter, pre-cooler . . . . 156

6.28 Fit result of Eq. (6.7) to different apertures and models, for the correction to infinite aperture, as a function of the intrinsic size of a cluster. NICMOS/NIC2 chip, F110W filter, pre-cooler . . . . 157

6.29 Fit result of Eq. (6.7) to different apertures and models, for the correction to infinite aperture, as a function of the intrinsic size of a cluster. NICMOS/NIC2 chip, F110W filter, post-cooler . . . 158

6.30 Fit result of Eq. (6.7) to different apertures and models, for the correction to infinite aperture, as a function of the intrinsic size of a cluster. NICMOS/NIC2 chip, F110W filter, post-cooler . . . 159

6.31 Fit result of Eq. (6.7) to different apertures and models, for the correction to infinite aperture, as a function of the intrinsic size of a cluster. NICMOS/NIC2 chip, F110W filter, post-cooler . . . 160

6.32 Fit result of Eq. (6.7) to different apertures and models, for the correction to infinite aperture, as a function of the intrinsic size of a given cluster. NICMOS/NIC2 chip, F160W filter, pre-cooler

6.33 Fit result of Eq. (6.7) to different apertures and models, for the correction to infinite aperture, as a function of the intrinsic size of a given cluster. NICMOS/NIC2 chip, F160W filter, pre-cooler

6.34 Fit result of Eq. (6.7) to different apertures and models, for the correction to infinite aperture, as a function of the intrinsic size of a given cluster. NICMOS/NIC2 chip, F160W filter, pre-cooler

6.35 Fit result of Eq. (6.7) to different apertures and models, for the correction to infinite aperture, as a function of the intrinsic size of a given cluster. NICMOS/NIC2 chip, F160W filter, post-cooler 164

6.36 Fit result of Eq. (6.7) to different apertures and models, for the correction to infinite aperture, as a function of the intrinsic size of a given cluster. NICMOS/NIC2 chip, F160W filter, post-cooler 165 
6.37 Fit result of Eq. (6.7) to different apertures and models, for the correction to infinite aperture, as a function of the intrinsic size of a given cluster. NICMOS/NIC2 chip, F160W filter, post-cooler 166

6.38 Fit result of Eq. (6.7) to different apertures and models, for the correction to infinite aperture, as a function of the intrinsic size of a given cluster. NICMOS/NIC2 chip, F205W filter, pre-cooler 167

6.39 Fit result of Eq. (6.7) to different apertures and models, for the correction to infinite aperture, as a function of the intrinsic size of a given cluster. NICMOS/NIC2 chip, F205W filter, pre-cooler 168

6.40 Fit result of Eq. (6.7) to different apertures and models, for the correction to infinite aperture, as a function of the intrinsic size of a given cluster. NICMOS/NIC2 chip, F205W filter, pre-cooler

6.41 Fit result of Eq. (6.7) to different apertures and models, for the correction to infinite aperture, as a function of the intrinsic size of a given cluster. NICMOS/NIC2 chip, F205W filter, post-cooler 170

6.42 Fit result of Eq. (6.7) to different apertures and models, for the correction to infinite aperture, as a function of the intrinsic size of a given cluster. NICMOS/NIC2 chip, F205W filter, post-cooler 171

6.43 Fit result of Eq. (6.7) to different apertures and models, for the correction to infinite aperture, as a function of the intrinsic size of a given cluster. NICMOS/NIC2 chip, F205W filter, post-cooler 172

6.44 Fit result of Eq. (6.7) to different apertures and models, for the correction to infinite aperture, as a function of the measured size of a cluster. PC chip, F555W filter . . . . . . . . . . . . . 174

6.45 Fit result of Eq. (6.7) to different apertures and models, for the correction to infinite aperture, as a function of the measured size of a cluster. PC chip, F555W filter . . . . . . . . . . . . . . 175

6.46 Fit result of Eq. (6.7) to different apertures and models, for the correction to infinite aperture, as a function of the measured size of a cluster. PC chip, F555W filter . . . . . . . . . . . . . 176

6.47 Fit result of Eq. (6.7) to different apertures and models, for the correction to infinite aperture, as a function of the measured size of a cluster. WF3 chip, F555W filter . . . . . . . . . . . 177

6.48 Fit result of Eq. (6.7) to different apertures and models, for the correction to infinite aperture, as a function of the measured size of a cluster. WF3 chip, F555W filter . . . . . . . . . . . 178

6.49 Fit result of Eq. (6.7) to different apertures and models, for the correction to infinite aperture, as a function of the measured size of a cluster. WF3 chip, F555W filter . . . . . . . . . . . . 179

6.50 Fit result of Eq. (6.7) to different apertures and models, for the correction to infinite aperture, as a function of the measured size of a cluster. ACS/WFC1 chip, F555W filter (chip 2 is equivalent) 180

6.51 Fit result of Eq. (6.7) to different apertures and models, for the correction to infinite aperture, as a function of the measured size of a cluster. ACS/WFC1 chip, F555W filter (chip 2 is equivalent) 181

6.52 Fit result of Eq. (6.7) to different apertures and models, for the correction to infinite aperture, as a function of the measured size of a cluster. ACS/WFC1 chip, F555W filter (chip 2 is equivalent) 182 
6.53 Fit result of Eq. (6.7) to different apertures and models, for the correction to infinite aperture, as a function of the measured size of a cluster. NICMOS/NIC2 chip, F110W filter, pre-cooler . . . . 183

6.54 Fit result of Eq. (6.7) to different apertures and models, for the correction to infinite aperture, as a function of the measured size of a cluster. NICMOS/NIC2 chip, F110W filter, pre-cooler . . . . 184

6.55 Fit result of Eq. (6.7) to different apertures and models, for the correction to infinite aperture, as a function of the measured size of a cluster. NICMOS/NIC2 chip, F110W filter, pre-cooler . . . . 185

6.56 Fit result of Eq. (6.7) to different apertures and models, for the correction to infinite aperture, as a function of the measured size of a cluster. NICMOS/NIC2 chip, F110W filter, post-cooler . . . 186

6.57 Fit result of Eq. (6.7) to different apertures and models, for the correction to infinite aperture, as a function of the measured size of a cluster. NICMOS/NIC2 chip, F110W filter, post-cooler . . . 187

6.58 Fit result of Eq. (6.7) to different apertures and models, for the correction to infinite aperture, as a function of the measured size of a cluster. NICMOS/NIC2 chip, F110W filter, post-cooler . . . 188

6.59 Fit result of Eq. (6.7) to different apertures and models, for the correction to infinite aperture, as a function of the measured size of a given cluster. NICMOS/NIC2 chip, F160W filter, pre-cooler

6.60 Fit result of Eq. (6.7) to different apertures and models, for the correction to infinite aperture, as a function of the measured size of a given cluster. NICMOS/NIC2 chip, F160W filter, pre-cooler

6.61 Fit result of Eq. (6.7) to different apertures and models, for the correction to infinite aperture, as a function of the measured size of a given cluster. NICMOS/NIC2 chip, F160W filter, pre-cooler

6.62 Fit result of Eq. (6.7) to different apertures and models, for the correction to infinite aperture, as a function of the measured size of a given cluster. NICMOS/NIC2 chip, F160W filter, post-cooler 192

6.63 Fit result of Eq. (6.7) to different apertures and models, for the correction to infinite aperture, as a function of the measured size of a given cluster. NICMOS/NIC2 chip, F160W filter, post-cooler 193

6.64 Fit result of Eq. (6.7) to different apertures and models, for the correction to infinite aperture, as a function of the measured size of a given cluster. NICMOS/NIC2 chip, F160W filter, post-cooler 194

6.65 Fit result of Eq. (6.7) to different apertures and models, for the correction to infinite aperture, as a function of the measured size of a given cluster. NICMOS/NIC2 chip, F205W filter, pre-cooler

6.66 Fit result of Eq. (6.7) to different apertures and models, for the correction to infinite aperture, as a function of the measured size of a given cluster. NICMOS/NIC2 chip, F205W filter, pre-cooler

6.67 Fit result of Eq. (6.7) to different apertures and models, for the correction to infinite aperture, as a function of the measured size of a given cluster. NICMOS/NIC2 chip, F205W filter, pre-cooler

6.68 Fit result of Eq. (6.7) to different apertures and models, for the correction to infinite aperture, as a function of the measured size of a given cluster. NICMOS/NIC2 chip, F205W filter, post-cooler 198 
6.69 Fit result of Eq. (6.7) to different apertures and models, for the correction to infinite aperture, as a function of the measured size of a given cluster. NICMOS/NIC2 chip, F205W filter, post-cooler 199

6.70 Fit result of Eq. (6.7) to different apertures and models, for the correction to infinite aperture, as a function of the measured size of a given cluster. NICMOS/NIC2 chip, F205W filter, post-cooler 200 


\section{Chapter 1}

\section{Introduction \& Overview}

\section{$1.1 \quad$ Introduction}

Humans have always been fascinated by the sky, the planets, the stars and other objects like "nebulae" (some of them later found to be galaxies, sometimes very similar to our own Milky Way). Apart from the practical side (such as navigation), this fascination is caused by prime philosophical questions such as Where do we come from? What happened before our existence? Where are we going to?, embedding astronomy into many ancient religions. The recently found sky disc of Nebra (made around 3600 years ago) illustrates this milleniaold interest, as do Stonehenge, the ancient Latin-American observatories or the sun cult of the Egyptian god Ra, to name a few.

In the late middle ages, scientific astronomical research began to emerge. At first, it primarily concerned the solar system (related to the work by Copernicus, Brahe, Kepler, Galilei, Newton). The first systematic investigation of "nebulae" was conducted by Messier in the years 1758-1782. Now many of these "nebulae" are known to be either galaxies or clusters of stars in our own Galaxy (the Milky Way).

The nature of these "nebulae" was discovered in the $20^{\text {th }}$ century, based on the ground-breaking work on the cosmological distance scale by Edwin Hubble (and favoured by new observatories and observational techniques). Now it is basic knowledge that the sun is part of a galaxy we call the "Milky Way", and that there is a large number of other galaxies distributed all over the sky, from nearby galaxies of the so-called "Local Group" to just-forming galaxies at the largest distances we can observe. These galaxies can be classified as different types. This classification can be based, e.g., on morphology (disky spiral galaxies just like our Milky Way, more spherical elliptical galaxies, and amorphous types), mass/brightness (from low-mass, low-luminosity dwarf galaxies like the Galaxy's satellite galaxies [the Magellanic Clouds] to bright and massive galaxies), environment (isolated galaxies, galaxy pairs presently in the process of merging with each other, gravitationally bound groups or clusters of galaxies) et cetera.

To understand the principles of galaxy formation and evolution one would ideally observe galaxies in the process of their formation at young ages of the Universe (and therefore at large distances from us), and follow them all along 
their evolutionary path to their present ages. Using state-of-the-art observatories like the Hubble Space Telescope (HST) or ESO's Very Large Telescope $(V L T)$, the detection of such just-forming galaxies is possible. However, even if we use such advanced technology, the lack of spatial resolution restricts observations to integrated properties of the galaxies as a whole.

On the other hand, nearby galaxies are more complex systems, containing populations of stars of different ages and varying degrees of chemical enrichment from different episodes of star formation during their life time. In addition, they have large reservoirs of gas, dust, black holes, the exotic "dark matter" component, et cetera. In principle, this mixture of different stellar populations provides us with information about the formation of these galaxies and about various processes within the galaxies during their life time (like galaxy merger events, accretion of intergalactic gas onto the galaxies, quiescent periods of star formation et cetera). In reality, the various effects are difficult to distinguish. Several methods can be employed to disentangle the multitude of stellar populations: (i) Integrated galaxy light contains contributions from different populations. The main complication arising from this complex mixture is that populations of different ages and metallicities contribute very different amounts of light in different wavelength regions. Its analysis requires either many ad hoc assumptions or a wealth of multi-wavelength observational data, or both. (ii) Resolved stellar population studies are possible only for the few very closest systems - and only the least dense regions - where we can distinguish individual stars, thanks to the high spatial resolution of the Hubble Space Telescope (HST). (iii) Star clusters (SCs), on the other hand, are very useful in this respect, as star cluster formation is a direct tracer of violent single star formation episodes. They are brighter than most single stars and their integrated properties can easily be studied to much greater distances (up to $\sim 300$ million light years $=$ $100 \mathrm{Mpc}$ ). Fortunately, crowding of SCs is usually not a serious issue. Hence, it is generally possible to study each cluster individually. Moreover, SCs are simple systems, and modelling them requires fewer assumptions than the case of integrated galaxy light: Each star cluster is formed almost instantaneously by the gravitational collapse of a single chemically well-mixed giant molecular cloud (GMC). Thus, all stars in a given SC have (almost) the same age and chemical composition.

In the Galaxy, star clusters are traditionally categorised into two distinct groups: Globular clusters (GCs), on the one hand, are thought to be old and formed at the onset of their host galaxy's formation (roughly the age of the Universe, approximately $12-13$ billion years $[=\mathrm{Gyr}])$ and of high mass $\left(10^{4}\right.$ - $10^{6}$ solar masses $\left.=M_{\odot}\right)$. They can be subdivided into metal-poor clusters associated with the Galactic halo and more metal-rich clusters associated with the bulge of the Galaxy. Open clusters, on the other hand, are thought to be:

- of low mass: $10^{2}-10^{4} M_{\odot}$

- young: typically younger than 1 billion years. Due to their lower masses dynamical disruption acts more strongly and is significant on shorter time scales than for GC-type objects.

- primarily associated with star formation processes in the now metal enriched Galactic spiral arms. 
However, one long-standing question still remains unanswered: Whether this distinction is grounded on fundamental differences of the two "classes" or originates from dynamical effects and/or different environmental conditions during cluster formation. For instance, the latter assumption could be based on the scenario that low-mass clusters produced during GC formation are already dynamically disrupted, while at present the conditions (e.g. gas density/pressure, overall star formation rate) inhibit/disfavour the formation of clusters with masses comparable to typical GC masses. The discovery of old open clusters (located in the outer, less dense regions of the Galactic halo, e.g. the clusters Berkeley 17, Melotte 66, NGC 188) seems to support this scenario, yet the final solution is still to be found.

In interacting and merging galaxies, bursts of intense star and star cluster formation are triggered, provided that sufficient gas reservoirs are available. These secondary SCs form from gas pre-enriched in the merging galaxies. SC formation is found to be an important, if not the dominant mode of star formation in gas-rich galaxy mergers. At least some fraction of the newly formed SCs will survive for as long as several billion years and evolve into GC counterparts, as evidenced by $1-3$ Gyr old clusters in merger remnant galaxies. The relation between these so-called Young Massive Clusters (YMCs) on the one hand and globular and open clusters on the other has remained unclear so far.

The survival of a star cluster does not only depend on its environment, mass and size, but most crucially on its stellar content: As high-mass stars evolve faster and more energetically than low-mass stars, a star cluster deficient in low-mass stars will be destroyed by the energy input of the evolving and dying high-mass stars. Only a sufficient number of low-mass stars can prevent a star cluster from disruption. SCs, and GCs in particular, represent a fossil record of the conditions in their host galaxies at the time of their formation. They are modified by stellar evolutionary processes which nowadays are generally well understood, as well as by internal and external disruption and evaporation processes. The age and metallicity distributions of SC systems are therefore key clues of the evolutionary history of their parent galaxies.

One way to study star clusters from their spatially integrated properties is to use evolutionary synthesis modelling. For such models the composition of a stellar population, i.e. the number of stars of a given mass, age, and metallicity, is computed at each time step. The corresponding spectra of all stars are summed up, to give a model grid of integrated spectra as a function of the age of the stellar population and its metallicity. By convolving the integrated spectra with filter response functions, artificial magnitudes and colours are synthesised, which can be compared with observed magnitudes and colours. For galaxies or other composite stellar populations, their star formation history has to be taken into account whereas star clusters are characterised by the formation all their stars in a single short $\left(\lesssim 10^{5}\right.$ years) burst (i.e. in one time step of the evolutionary synthesis models $=4$ million years $[=\mathrm{Myr}])$.

The study of young star clusters in interacting and merging galaxies has only recently emerged, owing to the exquisite observing conditions of the HST. Similar to the studies of genuinely old GCs, the study of young SCs has its own caveats. To name a few:

- Young, especially still-forming SCs are surrounded by clouds of ionised gas (remnants of their parental gas clouds), leading to contributions from 
emission lines and continuum emission to the integrated cluster photometry. The inclusion of this gas emission is not yet standard in evolutionary synthesis models, since its handling is non-trivial and various approaches are followed at present.

- To determine physical parameters of the clusters (age, mass, metallicity, extinction within the host galaxy) from their integrated photometry, one usually compares certain observed colours (magnitude differences as observed in different filters of the same cluster) with predictions from evolutionary synthesis models. As to young SCs, the time evolution of these colours is much faster and contains certain jumps (due to the occurrence of certain features in stellar evolution at certain times, e.g. the thermallypulsing AGB phase for ages of 0.1 - 1 Gyr, the first occurrence of RGB stars, et cetera). An objective and well-tested algorithm to determine the cluster parameters and their uncertainties, taking into account all the available information, is therefore of prime significance.

- Young star clusters (especially YMCs) are preferentially found in highlyactively star-forming galaxies like starburst galaxies and interacting / merging galaxies. The high level of ongoing star formation causes problems with

- stellar contamination: Distinguishing single stars from star clusters can be difficult as a single bright $\mathrm{O}$ star or supergiant can be as bright as a whole star cluster of low mass and/or older age. Depending on the depth of the exposures and the age of the observed starburst (hence the luminosity of the brightest still living star), this is relevant up to distances $\lesssim 20 \mathrm{Mpc}$.

- background contamination: Because of the fast evolution of cluster colours and the finite length of a starburst, young SCs span a wide range of colours, making the removal of background galaxies more difficult. However, in general, young SCs are concentrated towards the centres of their host galaxies where those galaxies are mostly opaque and background sources are unlikely to be discovered.

- variable galaxy background: In such highly-actively star-forming galaxies, the galaxy's unresolved background light as well as the distribution of dust within the host galaxy are often very inhomogeneous, hampering cluster selection, accurate photometry and reliable observational completeness determinations.

\subsection{Overview}

To tackle these above-mentioned problems, U. Fritze - v. Alvensleben and R. de Grijs initiated the project leading to this $\mathrm{PhD}$ thesis.

After having made myself familiar with the evolutionary synthesis code GALEV (developed by U. Fritze - v. Alvensleben and regularly updated with the best available input physics), I started my work by including the effects of gaseous emission into the GALEV code. The results of this project include 
1. the relative contribution of the gaseous emission (emission lines, continuum emission and total emission) to the total flux

2. the resulting changes in cluster magnitudes and colours

3. the extension of the models towards younger ages (starting now at $4 \mathrm{Myr}$ while previous models started at $140 \mathrm{Myr}$ )

4. the calculation of cluster magnitudes in numerous additional filter systems, especially HST filter sets, to reduce the inaccuracies inevitably inherent in any transformations between different filter sets.

The results are presented in Chapter 2 and published in Anders \& Fritze - v. Alvensleben, 2003, A\&A, 401, 1063. Since the publication of the results, minor changes in the input physics and the programme code have led to minor changes of the results. These changes are already included in Chapter 2 and are presently prepared to be published as an erratum.

In order to utilise the new models to interpret observed cluster photometry, a robust tool to compare models with observations was necessary. Therefore I started a collaboration with N. Bissantz, a statistician with astrophysical background from the Institute of Mathematical Stochastics at the University of Göttingen, to put this tool onto solid statistical ground. With his help I developed an algorithm (we named it the "ANALYSED tool") to determine the best-matching set of physical parameters for each cluster including the $1 \sigma$ uncertainty ranges of each model parameter. Both observational and model-inherent errors are taken into account by the algorithm. After having developed this algorithm, I carefully evaluated the reliability and reproducibility of the new method, using a vast number of artificial cluster tests. These tests cover the entire parameter space of the models and include the following questions:

- Which filters/filter combinations are particularly well/poorly suited to provide reliable cluster parameter estimates?

- How do certain (justified or wrong) a priori restrictions of the parameter space used during the analysis affect the results?

- Which effects arise from finite observational uncertainties?

To our best knowledge, the ANALYSED tool is by far the most advanced algorithm of its kind, and no comparable systematic study on parameter determination uncertainties was ever published before. The results are presented in Chapter 3 and published in Anders, Bissantz, Fritze - v. Alvensleben \& de Grijs, 2004, MNRAS, 347, 196.

Besides this paper, I got involved in further studies aimed at quantifying the reliability of utilising evolutionary synthesis models to derive cluster parameters from observations. In this context the "cluster fitting challenge" is of relevance. This international project provided different groups with the same data sets. The groups were asked to analyse these data sets by using their own methods and models. Subsequently, we compared the results of the different groups (see Sect. 8.3). In another project we compared results obtained from integrated 
photometry with those obtained from isochrone fitting of colour-magnitude diagrams (CMDs). We made use of two independent homogeneous data sets of clusters in the Large Magellanic Cloud (LMC; see Sect. 8.2).

By having established the theoretical background, I applied previous theoretical lessons to observations. I chose the nearby dwarf starburst galaxy NGC 1569 for three reasons:

1. It is nearby, and therefore clusters are clearly extended (though not resolved into single stars; this eases the distinction between single stars and clusters)

2. The galaxy is presently undergoing (or just finishing) a strong starburst. Hence, clusters formed in this event are young and a fair number of such clusters is expected.

3. It contains two young massive star clusters with spectroscopically confirmed high masses (with masses larger than average GCs in the Milky Way), although the remaining cluster system is almost unstudied.

Studying a cluster sample roughly four times as large as any previous sample provides new insights into the star cluster formation processes in this interesting galaxy. The results are presented in Chapter 4 and published in Anders, de Grijs, Fritze - v. Alvensleben \& Bissantz, 2004, MNRAS, 347, 17. The work on the cluster system of NGC 1569 was chosen to accompany a joint ESA/NASA photo press release. The image was kindly produced by L. Lindbergh Christensen at ESO, and reproduced ((together with the original press release text) in Sect. 5, under the permission of ESA and NASA.

I applied the new methods to cluster samples in further galaxies, strengthening present collaborations. The abstracts of the resulting papers are presented in Chapters 8.4-8.7.

After having finished the work on the cluster system of NGC 1569 an idea on how to improve the photometry of extended sources (especially spherical sources like star clusters) emerged during discussions.

Due to diffraction and charge diffusion effects (and seeing effects caused by the Earth's atmosphere, in the case of ground-based observations), the image of a point source appears extended. This artificially extended image is called the "Point Spread Function", or PSF, of an instrument. The total flux (or magnitude) of a point source can be measured by fitting the observed light profile with the appropriate PSF. This method is not applicable to extended objects such as nearby star clusters! Star cluster photometry is usually done by "aperture photometry": around the source a circle is defined, containing (presumably) all the flux originating from the source. In addition, an outer annulus is defined to estimate the local value of the sky background. The sky background is the flux contribution from the surrounding galaxy light rather than from the source itself. There are two ways of defining these apertures: 1) using the same apertures for all clusters of a given cluster sample and set of observations, 2) by visually inspecting each cluster and assigning individual apertures to each cluster, depending on the apparent size and surrounding region of the cluster. Ideally, the source aperture should be as large as possible to contain the complete source flux.

In reality, both approaches have their advantages and drawbacks: 
- The generic apertures' approach

+ is fast and easily applicable

+ gives well-defined, easily reproducible results and allows reliable estimates of observational completeness

- underestimates the flux of larger sources with respect to the flux of smaller sources: in the former case more source flux resides outside the source aperture, and is erroneously assigned as background flux

- is more affected by strongly variable background fields and neighbouring sources contaminating source and/or sky fluxes. This contamination by neighbouring sources is usually referred to as "crowding".

- The individual apertures' approach

+ can assign apertures in a way to avoid contaminating sources and to contain the largest possible fraction of source flux, regardless of source size

- gives more subjective, not easily reproducible results, especially not well suited to determine the observational completeness easily (if at all)

To provide advances in this field of aperture photometry, R. de Grijs, M. Gieles (University of Utrecht, NL) and I started studying cluster size measurements. Subsequently, I employed this size information to calculate the cluster's flux fraction missed by using finite apertures (so-called "aperture corrections"). In Chapter 6 (and in P. Anders, M. Gieles \& R.de Grijs, A\&A, in press) I provide an easily applicable "cookbook" for observers to improve their photometry of extended sources and to measure the sizes of their objects. As this issue is most important for the high spatial resolution of space-based cluster observations, I focus my work on facilities on-board the HST. Using artificial cluster tests, I quantify the impact of

- cluster brightness

- different cluster light profiles

- choice of camera/chip/position on the chip

- choice of filter

- sky noise

- fitting radius

on the size determination. Considering the obtained size information, I calculate aperture corrections and the fraction of cluster light wrongly assigned as sky flux (both as a function of cluster size) and compare the limitations of my method to those of a more widely used method to measure source sizes.

I first applied the new methods for size determinations and aperture corrections to the young star cluster system of the nearest major galaxy merger, the "Antennae" galaxies (NGC 4038/39). 
In section 7 I present first results related to the distribution of cluster luminosities (the luminosity function) of these YMCs. Previous studies found a power-law luminosity function for these YMCs, for the Antennae system as well as for other, less actively star-forming environments. In contrast to this power-law luminosity function for YMCs the luminosity function of old globular clusters is known to be Gaussian-shaped. The parameters of this Gaussian seem to be universal when globular cluster systems of different galaxies are compared. The detection of the turnover of the Gaussian in an actively star-forming galaxy is severely complicated by strongly variable sky background and completeness functions.

We put great effort into objective and reproducible source selection, photometry and especially the observational completeness determination. Furthermore, in collaboration with N. Bissantz und L. Boysen from the Institute of Mathematical Stochastics at the University of Göttingen, we built a statistically robust tool to investigate the shape of luminosity functions, taking reliably into account the distribution of observational uncertainties and the observational completeness.

The thesis finishes with a summary of the presented research results and an outlook of work in progress and/or in preparation. 


\section{Chapter 2}

\section{Spectral and Photometric Evolution of Young Stellar Populations: the Impact of Gaseous Emission at Various Metallicities ${ }^{1}$}

\section{$2.1 \quad$ Introduction}

In Schulz et al. (2002) we presented spectral evolutionary synthesis models for SSPs of a wide range of metallicities $0.02 \leq Z / Z_{\odot} \leq 2.5$. Our models are based on isochrones from the Padova group that include the TP-AGB phase and on the spectral model atmosphere libraries from Lejeune et al. (1997, 1998), rectified to yield agreement with observed colors from $U$ through $K$ of stars with effective temperatures in the range $2000 \mathrm{~K}$ through $50000 \mathrm{~K}$. Stars with higher temperatures are treated as black body radiators. These models give the time evolution of spectra as well as of luminosities and colors in a large set of filter systems. As they did not include gaseous emission important during the lifetime of massive ionising stars, they cover a range of ages from $140 \mathrm{Myr}$ through 14 Gyr.

SSP model results are useful not only for analyses of star clusters, the genuine SSPs, but are also readily superposed to describe the evolution of composite stellar populations like galaxies with star formation histories extended in time and with various chemical enrichment histories. They can also directly be combined with cosmological structure formation scenarios that include a star formation criterium.

Systems with active ongoing star formation like very young star clusters, actively star-forming or star-bursting galaxies in the local as well as in the

\footnotetext{
${ }^{1}$ This chapter was published as Anders \& Fritze - v. Alvensleben, 2003, A\&A, 401, 1063. With respect to the published version there are some changes due to changes in the input physics in the mean time.
} 
high-redshift Universe, protogalaxies and subgalactic fragments in particular, require the inclusion of gaseous emission. This is not only important for the characteristic emission lines in the spectra but gaseous emission, both in terms of lines and continuum, give important contributions to broad band luminosities and colors (cf. Krüger et al. 1995, Zackrisson et al. 2001).

The wealth of HST ACS data currently becoming available - on many very young star cluster systems in particular - prompted us to not only include the gaseous emission in an updated way into our spectral evolutionary synthesis models for SSPs of various metallicities but also to provide versatile tables for the luminosity and color evolution of these SSPs in ACS filter bands.

Results of these models have already been proven very useful in the interpretation of the ACS Early Release Observations of the very young star cluster systems in the Mice and Tadpole galaxies (de Grijs et al. 2002).

\subsection{Input Physics}

\subsubsection{SSP Models}

Except for the gaseous emission that was not yet included in our SSP models before, we use the same input physics as presented in detail in Schulz et al. (2002). This includes isochrones from the Padova group containing the TP-AGB phase and model atmosphere spectra from Lejeune et al. (1997, 1998), extending from $90 \AA$ through $160 \mu \mathrm{m}$, for five different metallicities $Z=0.0004,0.004$, $0.008,0.02=\mathrm{Z}_{\odot}$ and 0.05 or $[\mathrm{Fe} / \mathrm{H}]=-1.7,-0.7,-0.4,0$ and +0.4 . Inclusion of the TP-AGB phase has been shown to be very important for colors like $V-I$ and $V-K$. As we have shown in Schulz et al. (2002), age-dating of star clusters on the basis of their $V-I$ colors, as often done for young cluster systems in interacting galaxies or merger remnants, can go wrong by a factor 2 (at fixed metallicity and extinction) if the TP-AGB phase is neglected in the models.

We present our results for a Salpeter and a Scalo IMF as in Schulz et al. (2002).

\subsubsection{Gaseous Emission}

Gaseous emission is primarily related to very hot stars, i.e. to massive stars in early evolutionary phases of an SSP. However, hot white dwarfs (WDs) can also contribute in later stages (but see next subsection). Gaseous emission depends on metallicity in a two-fold way. First of all, stars get brighter and hotter on average in stellar populations at lower metallicity. The lifetimes of low mass stars get shorter, those of high mass stars get longer at lower metallicities as compared to solar. This affects the output rates in terms of hydrogen ionising photons, $N_{\text {Lyc }}$, of a stellar population as well as its absorption line spectrum and, hence both the strengths of all gaseous emission lines in a uniform way and the gas continuum emission. Second, the chemical composition and the physical properties of the gas exposed to the same ionising radiation field determine the relative strengths of different emission lines.

The physical properties and the chemical composition of the gas ionised by a bunch of stars of given metallicity are not known a priori. They determine, however, the flux ratios of non-hydrogen element lines relative to $\mathrm{H}_{\beta}$. We therefore 
chose to assume that the gas has the same solar-scaled abundances as our single burst single metallicity stellar population and to use observationally determined emission line ratios for all non-hydrogen lines. In this respect our approach is similar to that of Pérez-González et al. (2002) who also use observed line ratios for their sample of strong $\mathrm{H}_{\alpha}$ emitting UCM survey galaxies. It differs from those of Charlot \& Longhetti (2001), Moy et al. (2001), or Zackrisson et al. (2001), who assume electron densities and temperatures for the gas at low metallicities and couple a photoionisation code to their evolutionary synthesis model.

On the basis of effective temperature and bolometric luminosity (and hence radius and surface gravity) of every star in a given isochrone, its flux of hydrogen ionising photons $\left(N_{\text {Lyc }}\right)$ is calculated from up-to-date non-LTE expanding model atmospheres that take into account line-blanketing as well as stellar winds and recent temperature and gravity calibrations (Schaerer \& de Koter 1997, Vacca et al. 1996, Smith et al. 2002). Summing up the $N_{\text {Lyc }}$ of all stars present in one isochrone gives the total $N_{\text {Lyc }}$ of the stellar population at a given time. Depending on the metallicity a fraction of this flux is absorbed by dust immediately ( $30 \%$ is assumed to be absorbed for metallicities $\geq 0.008$, no absorption is applied for lower-metallicity environments, following Mezger 1978, see also Weilbacher et al. 2000). From the remaining $N_{\text {Lyc }}$ flux of an isochrone we calculate the gaseous continuum emission and the hydrogen line fluxes as described in Krüger et al. (1995) and Weilbacher et al. (2000) assuming ionisation-recombination equilibrium (Osterbrock case B). The detailed formulae were already presented in Krüger et al. (1995). For instance, the $N_{\text {Lyc }}$ is converted into an $\mathrm{H}_{\beta}$ flux using

$$
\mathrm{F}\left(\mathrm{H}_{\beta}\right)=4.757 \times 10^{-13} \cdot \mathrm{N}_{\mathrm{Lyc}} .
$$

Emission line fluxes for elements other than $\mathrm{H}$ are calculated from the line ratios relative to $\mathrm{H}_{\beta}$, given in Table 1 .

For low metallicity gas, line ratios for an exhaustive set of strong forbidden and allowed transitions from UV through NIR are obtained from the extensive observational database of Izotov et al. (1994, 1997) and Izotov \& Thuan (1998), subdivided into the metallicity bins covered by our SSP model grid, as given in the Z1- and Z2-columns in Table 1 , for $Z=0.0004$ and $Z=0.004$ respectively.

Line ratios in reasonably metal-rich gas $\left(Z=0.008, Z_{\odot}\right.$, and $\left.Z=0.05\right)$ are taken from Stasińska (1984) as they have been shown to be in good agreement with Galactic HII region data (Sivan et al. 1986). No further distinction is made between the 3 metallicities $Z=0.008, Z_{\odot}$, and $Z=0.05$, as galactic HII regions do show this full range of metallicities and their line ratios at fixed metallicity show considerable scatter.

The line ratios, of course, do vary with electron temperature, number density and/or the filling factor of the ionized gas. However, we aim to describe typical environments at the different metallicities. We have investigated the dispersion of line ratios within each of our metallicity bins $Z 1, Z 2$ and $Z 3-Z 5$ and the impact on integrated magnitudes in the broad band filters we consider. We find that, on average, the scatter in the line ratios is $\lesssim 30 \%$ (with few exceptions for weak lines). This scatter translates into a scatter in magnitude of at most $0.2 \mathrm{mag}$ for solar metallicity, and up to $0.4 \mathrm{mag}$ for the lowest metallicity. Compared with an error of $0.4 \mathrm{mag}$ and $1.0 \mathrm{mag}$, respectively, by not taking gaseous emission into account at all, we consider this an improvement. However, 
we would like again to emphasize, that our models are meant to improve upon models neglecting the gaseous emission in early evolutionary stages in terms of broad band luminosities and colors. For star clusters etc. that have detailed spectral information to determine all their ionising parameters, photoionisation codes will allow for a more precise individual description of their line strengths and their impact on colors.

In including the gaseous line emission our models go beyond Leitherer et al. 's STARBURST99 models which do include the gaseous continuum but no emission lines. Smith et al. (2002) will soon provide an updated version of the STARBURST99 models that also include emission lines with particular focus on WR star diagnostics. We recall that while STARBURST99 is based on stellar evolution input physics from the Geneva group, our models use Padova isochrones.

\subsubsection{The role of white dwarfs}

The inclusion of white dwarfs (WD)s changes the magnitudes of passbands redwards of $U$ by up to few times $0.001 \mathrm{mag}$. UV- and FUV-passbands show changes of generally $0.01 \mathrm{mag}$. However, even these values are likely to be upper limits - at least in the description of star clusters - since their derivation is based on the assumption of the presence of a sufficiently large amount of interstellar matter (ISM) to be ionized.

In the case of WDs in star clusters, two aspects are of importance: First, WDs only appear after a delay of roughly $500 \mathrm{Myr}$, when SNe had more than enough time to blow away the interstellar material from a star cluster region. Second, the phase of planetary nebulae (PNe), when the region surrounding the WD is again refilled with gaseous material from the ejected envelopes until the ejected material is dispersed into the ISM, is fairly short in comparison with the time steps available $(\sim 25,000$ yrs vs. $4,000,000 \mathrm{yrs})$. AS a result, the filling factor (and hence the average density) of the ISM during phases when WDs could contribute to the emission line flux is expected to be well below 1 , resulting in a negligible contribution of gaseous emission due to WDs to broad band magnitudes. In addition, PNe in globular clusters seems to be surprisingly rare (Jacoby et al. 1997).

In the case of galaxies, two scenarios might occur: Either the galaxy is gaspoor, hence not much matter is available that could be ionized, and the situation is similar to that of star clusters with low or negligible contributions of WDs. Or the galaxy is gas-rich, in which case the emission is probably dominated by star formation anyway, as described with our models presented here.

\subsubsection{Filter Systems}

In addition to the Standard Johnson $U B V R I J H K L$, Thuan \& Gunn $g r$, Kron \& Koo $U^{+} J^{+} F^{+} N^{+}$, Washington $C M T 1 T 2$, Strömgren $u v$ b y, HST WFPC2 F160BW ... F F14W filters already included in Schulz et al. (2002), we here additionally include the HST WFPC2 medium band filters F410M, F467M and F547M, the NICMOS filters F110W, F160W, F205W and F222M, and the ACS/WFC broad band filters F435W . . . F850LP.

Calibrations for the ACS filters have been performed according to Gilliland 2002 (priv. comm.). 
Table 2.1: Non-hydrogen emission lines and their line strengths, normalized to $\mathrm{H}_{\beta}$ line strength, as a function of metallicity $(Z 1=0.0004, \mathrm{Z} 2=0.004$, $\left.\mathrm{Z} 3=0.008, \mathrm{Z} 4=0.02=Z_{\odot}, Z 5=0.05\right)$

\begin{tabular}{|c|c|c|c|c|}
\hline Line & $\lambda[\AA]$ & $\begin{array}{l}\frac{F_{L}}{F_{\mathrm{H}_{\beta}}} \\
Z 1\end{array}$ & $\begin{array}{l}\frac{F_{L}}{F_{\mathrm{H}_{\beta}}} \\
Z 2\end{array}$ & $\begin{array}{c}\frac{F_{L}}{F_{\mathrm{H}_{\beta}}} \\
Z 3-Z 5\end{array}$ \\
\hline$[\mathrm{CII}]$ & 1335.00 & 0.000 & 0.000 & 0.110 \\
\hline [OIII] & 1663.00 & 0.000 & 0.058 & 0.010 \\
\hline$[\mathrm{CIII}]$ & 1909.00 & 0.000 & 0.000 & 0.180 \\
\hline$[\mathrm{NII}]$ & 2141.00 & 0.000 & 0.000 & 0.010 \\
\hline [CII] & 2326.00 & 0.000 & 0.000 & 0.290 \\
\hline [MgII] & 2798.00 & 0.000 & 0.310 & 0.070 \\
\hline$[\mathrm{OII}]$ & 3727.00 & 0.489 & 1.791 & 3.010 \\
\hline [NeIII] & 3869.00 & 0.295 & 0.416 & 0.300 \\
\hline $\mathrm{H}_{\zeta}+\mathrm{HeI}$ & 3889.00 & 0.203 & 0.192 & 0.107 \\
\hline $\mathrm{H}_{\epsilon}+[\mathrm{NeIII}]$ & 3970.00 & 0.270 & 0.283 & 0.159 \\
\hline $\mathrm{HeI}$ & 4026.00 & 0.015 & 0.015 & 0.015 \\
\hline [SII] & 4068.60 & 0.005 & 0.017 & 0.029 \\
\hline$[\mathrm{SII}]$ & 4076.35 & 0.002 & 0.007 & 0.011 \\
\hline [OIII $]$ & 4363.00 & 0.109 & 0.066 & 0.010 \\
\hline $\mathrm{HeI}$ & 4471.00 & 0.036 & 0.036 & 0.050 \\
\hline$[\mathrm{ArIV}]+\mathrm{HeI}$ & 4711.00 & 0.010 & 0.014 & 0.000 \\
\hline [OIII $]$ & 4958.91 & 1.097 & 1.617 & 1.399 \\
\hline [OIII $]$ & 5006.84 & 3.159 & 4.752 & 4.081 \\
\hline$[\mathrm{NI}]$ & 5199.00 & 0.003 & 0.010 & 0.030 \\
\hline$[\mathrm{NII}]$ & 5755.00 & 0.000 & 0.000 & 0.010 \\
\hline $\mathrm{HeI}$ & 5876.00 & 0.096 & 0.108 & 0.140 \\
\hline$[\mathrm{OI}]$ & 6300.00 & 0.008 & 0.041 & 0.130 \\
\hline [SIII] & 6312.00 & 0.009 & 0.017 & 0.030 \\
\hline$[\mathrm{NII}]$ & 6548.05 & 0.005 & 0.059 & 0.136 \\
\hline [NII] & 6583.45 & 0.015 & 0.175 & 0.404 \\
\hline $\mathrm{HeI}$ & 6678.00 & 0.026 & 0.030 & 0.030 \\
\hline [SII] & 6716.00 & 0.037 & 0.188 & 0.300 \\
\hline [SII] & 6730.00 & 0.029 & 0.138 & 0.210 \\
\hline $\mathrm{HeI}$ & 7065.00 & 0.028 & 0.023 & 0.040 \\
\hline [ArIII] & 7135.79 & 0.027 & 0.071 & 0.035 \\
\hline$[\mathrm{OII}]$ & 7319.99 & 0.012 & 0.027 & 0.026 \\
\hline$[\mathrm{OII}]$ & 7330.73 & 0.007 & 0.014 & 0.014 \\
\hline [ArIII] & 7751.11 & 0.067 & 0.176 & 0.086 \\
\hline$[\mathrm{SIII}]$ & 9068.60 & 0.000 & 0.510 & 0.945 \\
\hline$[\mathrm{SIII}]$ & 9530.85 & 0.000 & 0.000 & 0.365 \\
\hline$[\mathrm{SII}]$ & 10286.73 & 0.000 & 0.000 & 0.048 \\
\hline [SII] & 10320.49 & 0.000 & 0.000 & 0.058 \\
\hline [SII] & 10336.41 & 0.000 & 0.000 & 0.054 \\
\hline
\end{tabular}




\subsection{Time Evolution of SSP Spectra with Gaseous Emission}

The new SSP models presented here extend to younger ages and have a better time resolution as compared to Schulz et al. (2002). Time resolution now is 4 Myr up to an age of $2.35 \mathrm{Gyr}$, and $20 \mathrm{Myr}$ for larger ages. The youngest age available now is $4 \mathrm{Myr}$ as compared to $140 \mathrm{Myr}$ in Schulz et al. . In Fig. 2.1 we present the early evolution of the UV - optical parts of SSP spectra of solar and low metallicity, respectively, for comparison. The much stronger emission lines in the low metallicity spectra are clearly seen as well as some differences in line ratios, as e.g. that of [OII]3727 relative to the adjacent Balmer lines $\mathrm{H}_{\delta}$ and $\mathrm{H}_{\gamma}$. The time evolution and metallicity dependence of the line strengths will further be discussed in Section 2.4. Fig. 2.2 shows spectra of a young low metallicity $(Z=0.0004)$ SSP over a longer wavelength range.

\subsection{Impact of Gaseous Emission on Broad Band Luminosities and Colors}

\subsubsection{Relative contributions of the gaseous emission - lines and continuum - to broad band fluxes}

In Fig. 2.3, the relative contributions of the gaseous emission to broad band fluxes in the Johnson passbands $U$. . . K are shown for some young ages, again for solar and low metallicity. The metallicity dependence visible in the $B$-band is due to strong oxygen and neon lines. $\mathrm{H}_{\beta}, \mathrm{H}_{\gamma}$ and $\mathrm{H}_{\delta}$ contribute significantly as well. The $V$-band contribution is dominated by strong oxygen lines (and to a lesser extent helium, nitrogen and sulphur), the strengths of which are metallicity dependent. The strong contribution in the $R$-band is caused by $\mathrm{H}_{\alpha}$, which is located close to the throughput maximum of the Johnson $R$ filter. The $I$-band is dominated by sulphur lines for high metallicities (predominantly the [SIII] $\lambda 9068.60$ and [SIII]9530.85 lines, both being moderately strong and close to the I-band throughput maximum), which are absent in the case of $Z=0.0004$. The remaining lines are weak or far from the maximum of the filter response function, resulting in a prominent dip in Figures 2.3/2.4 (lower panels). Only lines from the Paschen- and Brackett-series with small relative flux contributions are present in the NIR bands $J, H$ and $K$. Here, the contribution from the continuum emission becomes important.

To interpret the differences between Scalo and Salpeter IMF, as seen in Fig. 2.3 , two points are of importance: the contributions of the stars to the stellar continuum and the contributions to the ionising photons. The stellar continuum is dominated by stars around $9 M_{\odot}$, more or less independent of IMF and metallicity. However, the stars responsible for the ionising photons span a larger mass range in the low metallicity case as compared to solar metallicity, providing more ionising photons at low metallicity. In combination with the lower overall stellar luminosity for the Scalo IMF in comparison to the Salpeter IMF, this leads to an enhanced relative emission contribution for the low metallicity Scalo IMF, and a reduced contribution for the solar metallicity case, both compared to the respective Salpeter values. 

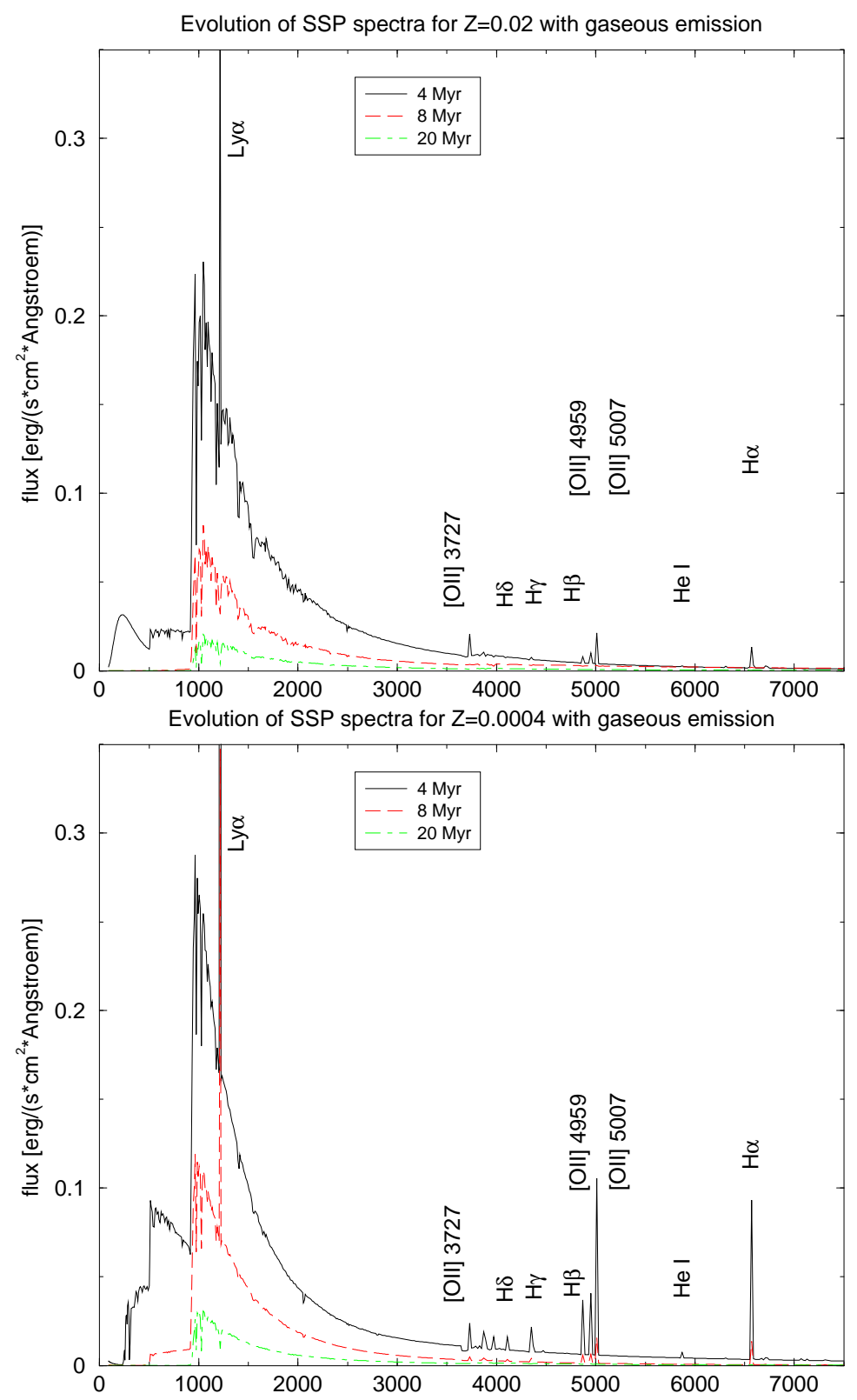

Figure 2.1: Spectrum in terms of flux $F_{\lambda}$ as a function of wavelength $\lambda$ at 3 different times for an SSP of solar metallicity (a) and metallicity $Z=0.0004$ (b), both with Salpeter IMF.

While Fig. 2.3 shows the overall emission contribution of the gas to broad band fluxes $U \ldots K$, this is decomposed into its line and continuum components in Fig. 2.4. This decomposition of the relative flux contribution of the gas to the fluxes in various filters shows that - for all metallicities - lines dominate in optical filters while continuum emission comes into play in the NIR bands. 


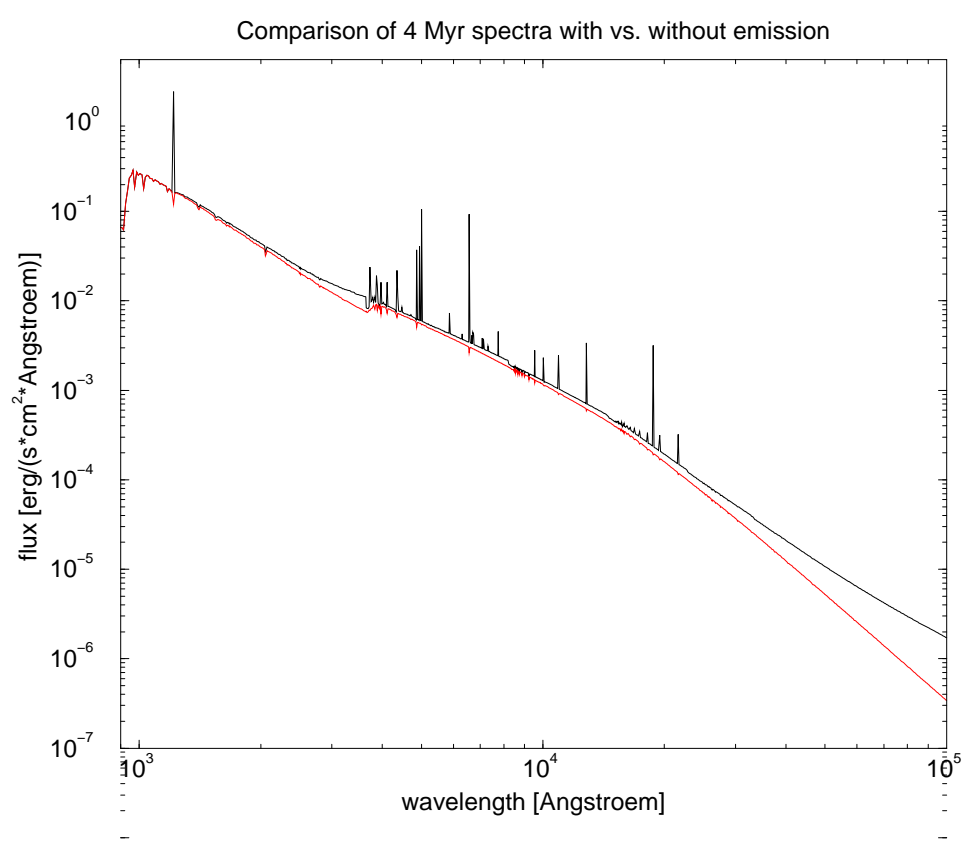

Figure 2.2: Spectrum in terms of flux $F_{\lambda}$ as a function of wavelength $\lambda$ for an SSP with $Z=0.0004$ and Salpeter IMF at an age of $4 \mathrm{Myr}$, with and without gaseous emission, in log-log presentation for better visibility.

A similar result was already reported by Krüger et al. (1995) in the context of evolutionary synthesis models for BCD galaxies. Like the hydrogen lines, the continuum emission is not directly metallicity-dependent, but indirectly via the higher ionising flux of lower metallicity SSPs and the dust absorption rate $f(Z)$ for ionising photons.

\subsubsection{Time evolution of gaseous emission contributions}

As can be seen from Fig. 2.5, the gaseous emission contribution is much stronger as well as longer-lasting at lower metallicity. In the Padova (as well as in Geneva) isochrones, the most massive stars have longer lifetimes at lower metallicities, as compared with solar. As discussed in Sect. 2.2.2, the population of massive stars gets hotter and more luminous, on average, at lower metallicities as compared to solar resulting in a higher output rate of ionising photons. In addition, the fraction of ionising photons directly absorbed by dust gets lower. Hence the relative contribution of gaseous emission e.g. to the $R$-band (dominated by $\mathrm{H}_{\alpha}$ ) starts from $15 \%$ at $Z_{\odot}$ and from $45 \%$ at $Z=0.0004$ at $4 \mathrm{Myr}$ and decreases by a factor 3 within roughly 6 and $11 \mathrm{Myr}$, respectively. At solar metallicity, the overall relative flux contribution of the ionised gas falls below $5 \%$ in all bands at ages $\leq 8 \mathrm{Myr}$. At our lowest metallicity, $Z=0.0004$, it falls below 5 $\%$ between roughly $11 \mathrm{Myr}$ and $14 \mathrm{Myr}$. 

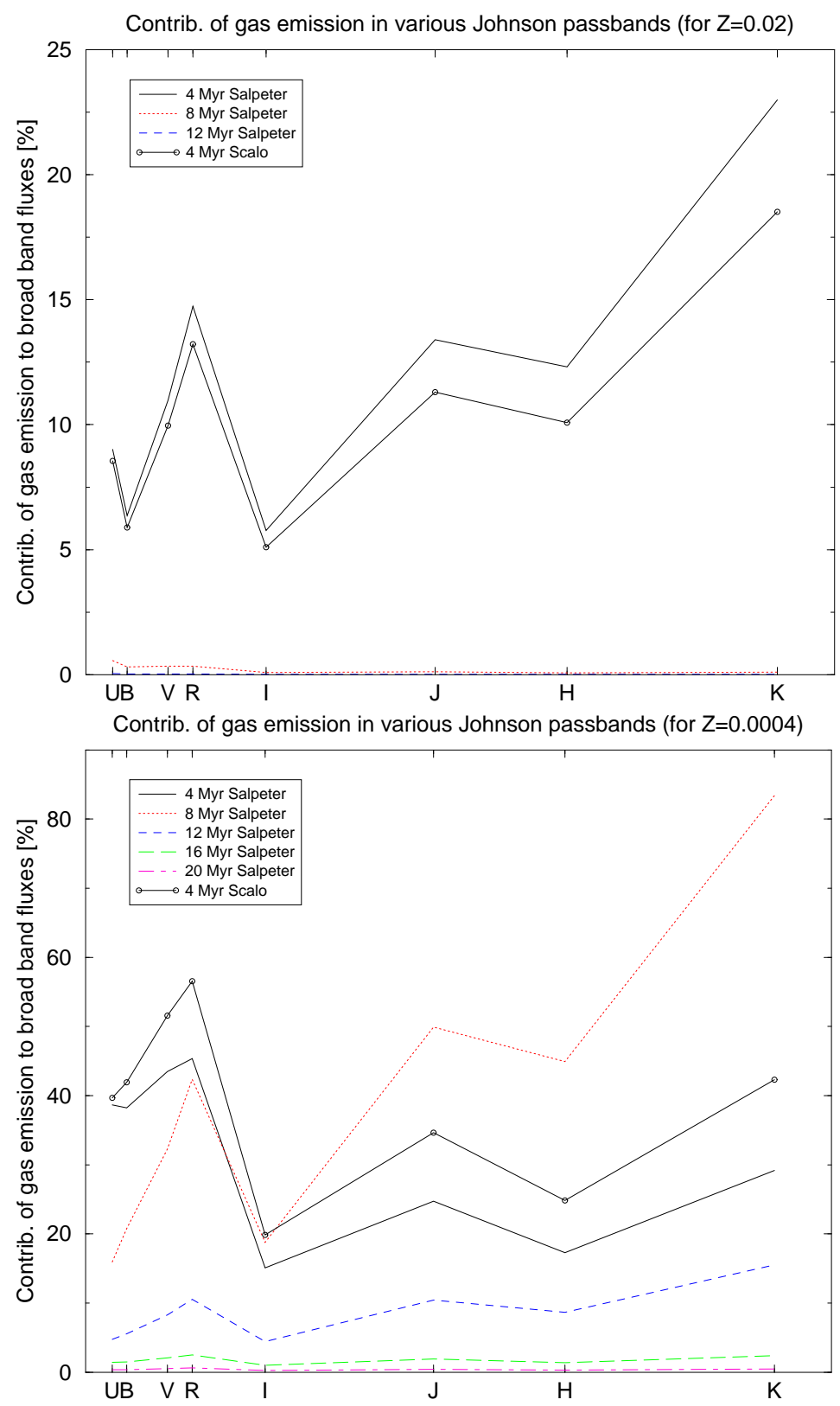

Figure 2.3: Relative contributions of the gaseous emission - lines and continuum - to broad band fluxes $U$. . . K at solar metallicity (upper panel) and low metallicity $Z=0.0004$ (lower panel).

\subsubsection{Effects of gaseous emission on the color evolution of SSPs}

As seen in Fig. 2.6 on the example of $V-I$ the inclusion of gaseous emission significantly affects the color evolution of SSPs at early stages, depending on 

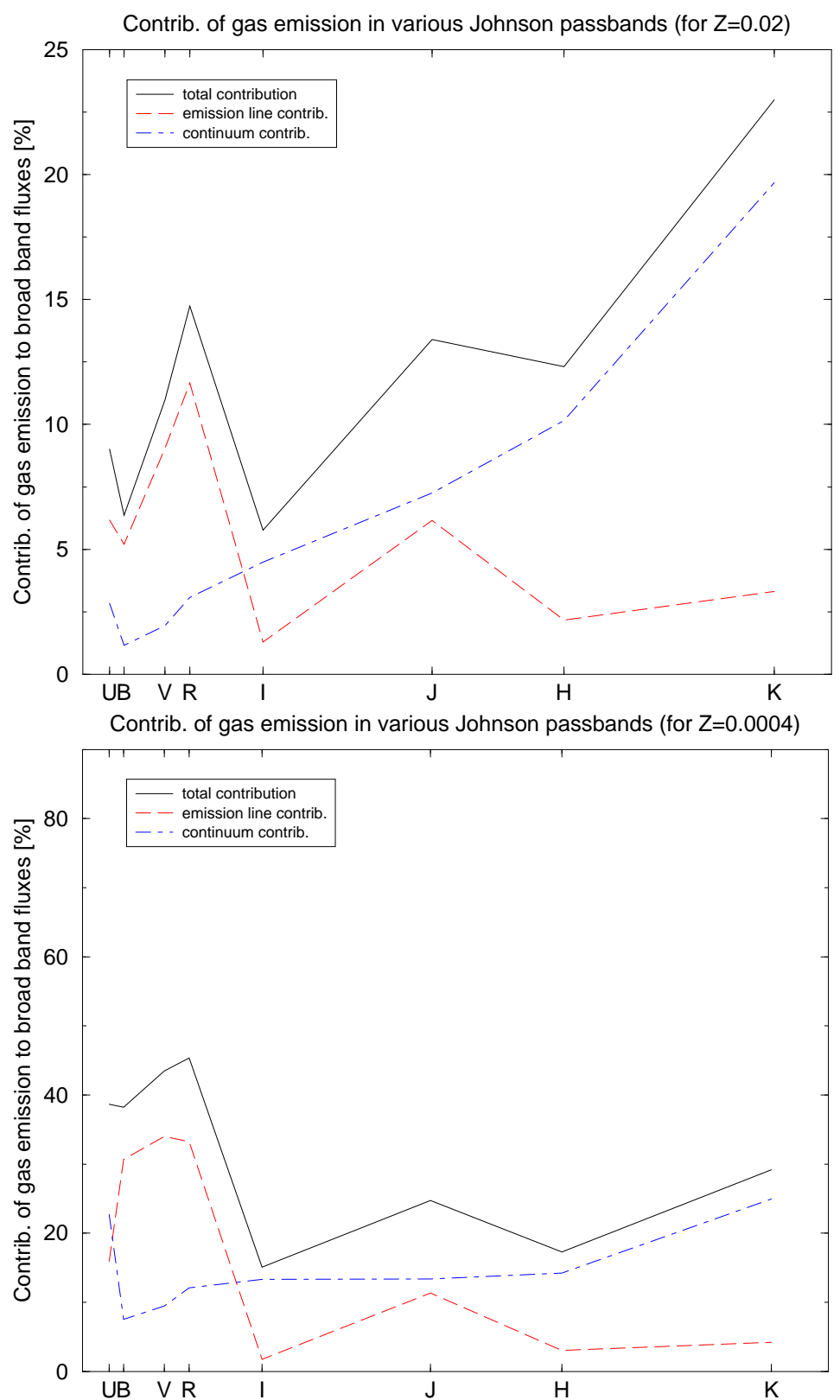

Figure 2.4: Decomposition of the total gaseous emission in terms of lines and continuum contributions in various filters bands $U$. . $K$ at an age of 4 Myr at solar metallicity (upper panel) and low metallicity $Z=0.0004$ (lower panel).

the metallicity, and the luminosity of a cluster of given mass, as can be seen in Fig. 2.7. This also, of course, affects the age and mass determinations from observed colors and luminosities.

From an observed $V-I=-0.2$ of a very young cluster, e.g., ages of 4 and 

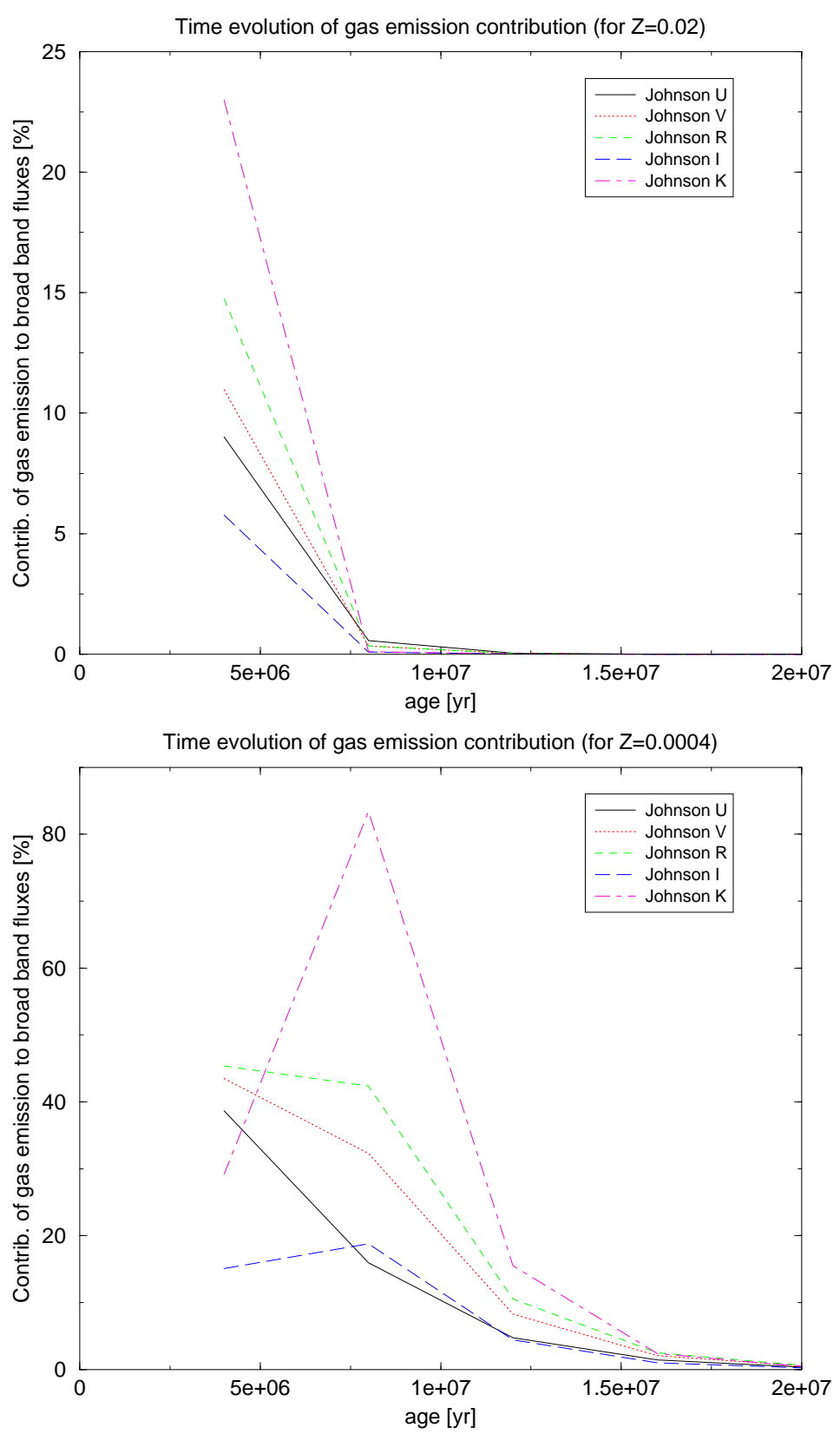

Figure 2.5: Time evolution of the gaseous emission contribution to broad band fluxes $U, B, V, I$, and $K$ at solar metallicity (a) and low metallicity $Z=0.0004$ (b).

$8 \mathrm{Myr}$ would be derived in case of solar and metallicity $Z=0.004$, respectively, if the effects of gaseous emission were not taken into account. Including them appropriately, the same observed color $V-I=-0.2$ would result in ages 4 and 7 or $12 \mathrm{Myr}$, for the two metallicities. This shows that ages estimated on the basis of $V-I$, without taking gaseous emission into account, for low 


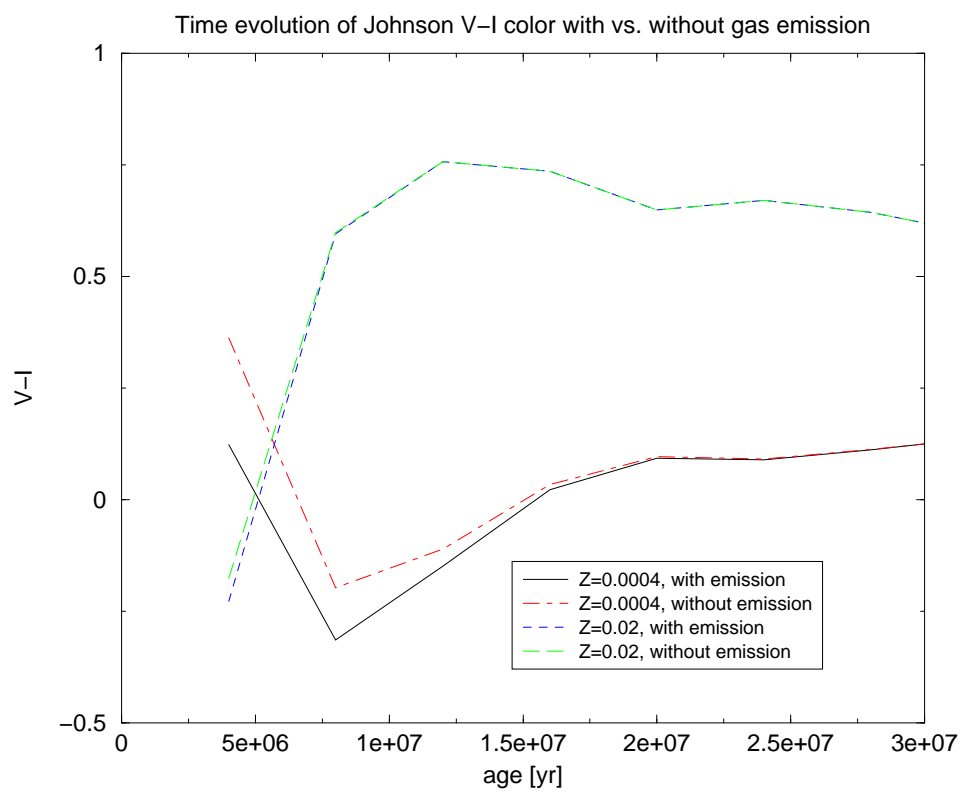

Figure 2.6: Time evolution of the Johnson colors $V-I$ with and without gaseous emission at solar metallicity $Z=0.02$ and low metallicity $Z=0.0004$.

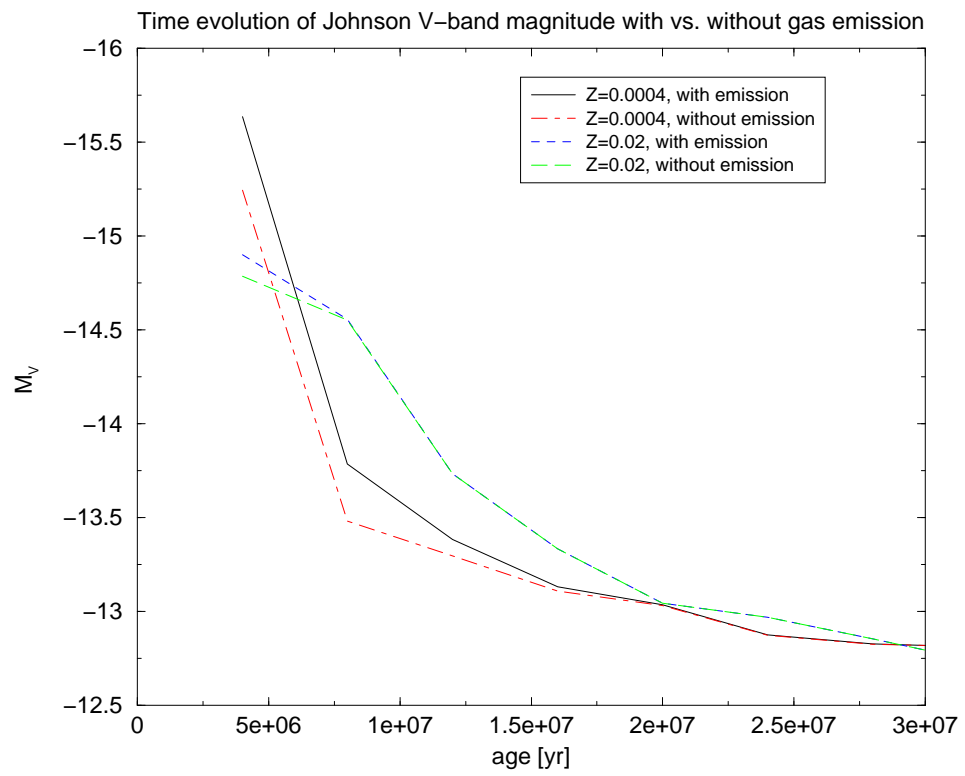

Figure 2.7: Time evolution of the magnitude in the Johnson $V$-band with and without gaseous emission at solar metallicity $Z=0.02$ and low metallicity $Z=0.0004$ for SSPs of the same mass.

metallicity clusters younger than $\sim 15$ Myr are underestimated by up to a factor $\sim$ 1.5. Differences decrease with increasing metallicity, and if gas emission is neglected at solar metallicity it has become negligible. In a similar way, masses 
estimated from the observed cluster $M_{V}$ with model mass-to-light ratios that do not account for gaseous emission contributions can be overestimated by a factor 1.5 at low metallicity.

\subsection{Discussion}

The aim of our models as presented here is to provide the spectral and photometric evolution of SSPs of various metallicities that also allow to describe the earliest stages of evolution when the ionising flux of a star cluster, a stellar (sub-)population etc. is strong enough to cause the surrounding gas to emit line and continuum radiation. Our motivation was that many HST observations of young star cluster systems in interacting and merging galaxies indeed do show such very young clusters with broad band colors that cannot be interpreted without accounting for their gaseous emission. Spectroscopy of some of those bright clusters has explicitly revealed their emission lines, e.g. Schweizer \& Seitzer (1998) in NGC 7252, Colina et al. (2002) in NGC 4303, Maoz et al. (2001) in NGC 1512 and NGC 5248, ...

We used most recent compilations of stellar output rates in ionising photons. Note that, while finding considerable differences in the output rates of Heionising photons, the latest and most sophisticated calculations by Smith et al. (2002) confirm the rates from Schaerer \& de Koter (1997) that we use for $\mathrm{H}$-ionising photons.

Our focus is on the impact of gaseous emission on the early luminosity and color evolution. For low metallicities $(Z \leq 0.004)$, we chose to use line ratios for the different metallicities of our SSP models as observed in HII regions of the respective metallicities. The line ratios for higher metallicities were taken from the theoretical models presented by Stasińska (1984). Hence, our models should not be used for any analyses of line ratios. For this purpose we recommend the use of models that couple to photoionization codes like those of Charlot \& Longhetti (2001) or Moy et al. (2001).

In Schulz et al. (2002) we gave evolutionary and cosmological corrections for our SSP models without gas for convenient use in cosmological structure formation models or any kind of models that build up galaxies with extended star formation (and possibly also chemical enrichment) histories. As the changes caused by the inclusion of gaseous emission are constrained to the earliest evolutionary stages $(t \lesssim 15 \mathrm{Myr})$ their effects on the cosmological and evolutionary corrections are only visible at redshifts extremely close to the redshift of the very onset of star formation. We therefore do not present cosmological and evolutionary corrections here and advise potential users to stay with the tables in Schulz et al. (2002).

Comparison of our GALEV model using Padova stellar input physics with results from Leitherer et al. 's (1999) STARBURST99 code, using Geneva stellar tracks, during the first Gyr of evolution covered by the latter shows fairly good agreement. As an example we show in Fig. 2.8 the time evolution of $B-V$. The differences are minor $(<0.1 \mathrm{mag})$, and mainly due to the better time resolution of the STARBURST99 code. Comparison of colors including longer wavelengths, e.g. $V-I$ or $V-K$, show larger deviations that are due to different input physics, especially a different treatment of red supergiants and the omission of the TP-AGB phase in STARBURST99. 


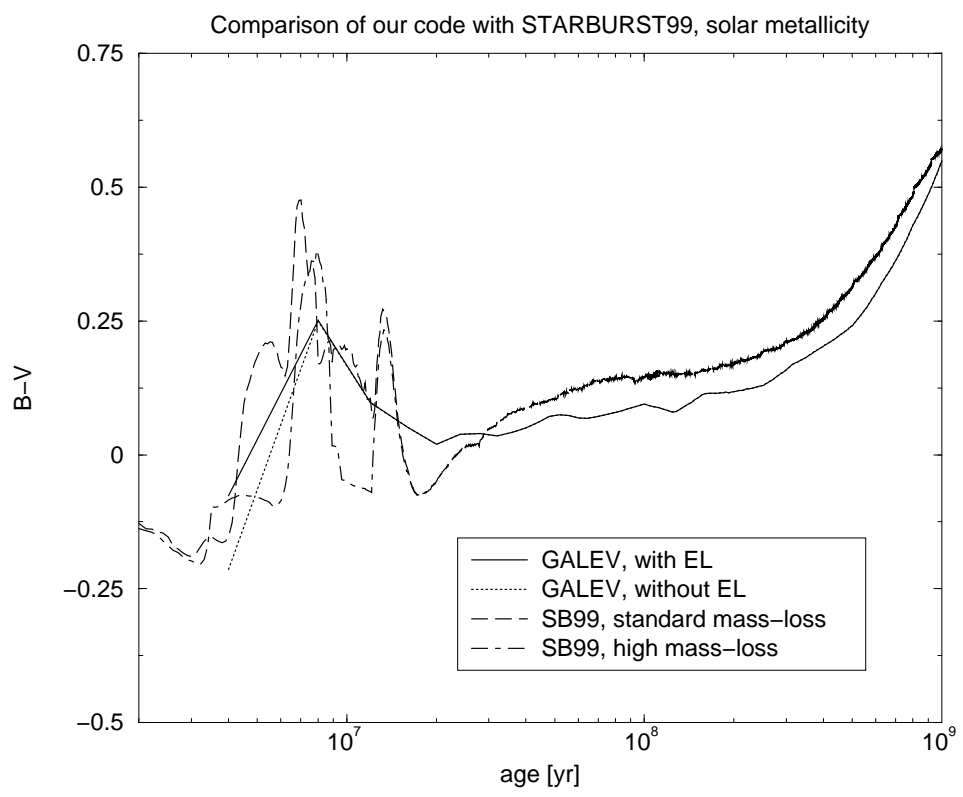

Figure 2.8: Comparison of our models with STARBURST99 models with different mass-loss rates, for solar metallicity and the Johnson color $B-V$.

\subsection{Structure and Description of Electronic Data- files}

Our model results are accessible at CDS and at http://www.astro.physik.unigoettingen.de/ galev/panders/SSPModels/index.html. The files have the following naming conventions:

- Each directory is labelled ssp_model_zx_y. Here $z x(x=1 \ldots 5)$ indicates the metallicity $(\mathrm{z} 1 \Leftrightarrow Z=0.0004, \mathrm{z} 2 \Leftrightarrow Z=0.004, \mathrm{z} 3 \Leftrightarrow Z=0.008, \mathrm{z} 4 \Leftrightarrow$ $Z=0.02, \mathrm{z} 5 \Leftrightarrow Z=0.05$ ), and $y$ the assumed IMF $\Phi(m) \sim m^{\alpha}$ ('salpeter' $\Leftrightarrow \alpha=-2.35$ for all masses; 'scalo' $\Leftrightarrow \alpha=-1.25$ for $m \leq 1 M_{\odot}, \alpha=-2.35$ for $1 M_{\odot}<m \leq 2 M_{\odot}$ and $\alpha=-3.00$ for $\left.2 M_{\odot}<m\right)$.

- Each directory contains a file named 'plotsgal.dat' containing the cluster mass as a function of age, a file named 'galspec.dat' containing the integrated cluster spectra as a function of age, and a file named 'magnitudes.dat' containing the integrated magnitudes of a cluster as a function of age in various passbands.

- Further information is included in the README file.

\subsection{Conclusions and Outlook}

We present an update of the evolutionary spectral synthesis models for SSPs of various metallicities presented in Schulz et al. (2002). Using the same stellar 
isochrones provided by the Padova group, including the TP-AGB phase important for $V-I$ and $V-K$ colors of SSPs in the age range $10^{8}$. . . $10^{9} \mathrm{yr}$, we add the gaseous emission contributions, both lines and continuum. They are very important not only for the spectral evolution but also for the photometric evolution in terms of broad band colors in early evolutionary stages, as long as ionising stars are alive. We use the best available output rates for $\mathrm{H}-$ ionising photons and observationally supported emission line ratios appropriate for the different metallicities. This allows us to extend our SSP models towards younger ages than accessible before. We provide an extensive set of electronic data for the time evolution of UV through NIR spectra of SSPs of metallicities $Z=0.0004,0.004,0.008,0.02=Z_{\odot}$, and 0.05 , for Salpeter and Scalo IMFs, from ages as young as 4 Myr all through 14 Gyr in timesteps of 4 Myr until an age of $2.35 \mathrm{Gyr}$ and of $20 \mathrm{Myr}$ thereafter. We also provide the luminosity and color evolutions in a large number of ground- and space-based filter systems including Johnson, Washington, HST WFPC2, NICMOS and ACS/WFC.

Our results also allow for easy superposition of SSPs of various ages - and possibly metallicities - to describe galaxies with all possible star formation and metal enrichment histories. They also are readily included into dynamical and cosmological galaxy or structure formation scenarios that contain a star formation criterium.

The evolutionary and cosmological corrections we gave in Schulz et al. (2002) are not affected by gaseous emission as it is constrained to stages earlier than $15 \mathrm{Myr}$, i.e. to redshifts extremely close to the redshift at which star formation sets in.

\subsection{Acknowledgements}

PA is partially supported by DFG grant Fr 916/11-1. We like to thank the anonymous referee for his or her suggestions that helped to improve the presentation of our results. 


\section{Bibliography}

Charlot S., Longhetti M., 2001, MNRAS, 323, 887

Colina L., Gonzalez Delgado R., Mas-Hesse J. M., Leitherer C., 2002, ApJ, 579, 545

de Grijs R., Lee J. T., Clemencia Mora Herrera C., Fritze - v. Alvensleben U., Anders P., 2003, New Astron., 8155

Gilliland R., STScI ACS Working Group, August-October 2002, priv. comm.

Izotov Y. I., Thuan T. X., Lipovetsky V. A., 1994, ApJ, 435, 647

Izotov Y. I., Thuan T. X., Lipovetsky V. A., 1997, ApJS, 108, 1

Izotov Y. I., Thuan T. X., 1998, ApJ, 500, 188

Jacoby G. H., Morse J. A., Fullton L. K., Kwitter K. B., Henry R. B. C., 1997, AJ, 114, 2611

Krüger H., Fritze - v. Alvensleben U., Fricke K. J., 1995, A\&A, 303, 41

Leitherer C., Heckman T. M., 1995, ApJS, 96, 9

Lejeune T., Cuisinier F., Buser R., 1997, A\&AS, 125, 229

Lejeune T., Cuisinier F., Buser R., 1998, A\&AS, 130, 65

Maoz D., Barth A. J., Ho L. C., Sternberg A., Filippenko A. V., 2001, AJ, 121, 3048

Mezger P. O., 1978, A\&A, 70, 565

Moy E., Rocca-Volmerange B., Fioc M., 2001, A\&A, 365, 347

Perez-Gonzalez P.G., Gil de Paz A., Zamorano J., Gallego J., Alonso-Herrero A., Aragon-Salamanca A., 2003, MNRAS, 338, 508

Perez-Gonzalez P.G., Gil de Paz A., Zamorano J., Gallego J., Alonso-Herrero A., Aragon-Salamanca A., 2003, MNRAS, 338, 525

Salpeter E. E., 1955, ApJ, 121, 161

Schaerer D., de Koter A., 1997, A\&A, 322, 598

Schulz J., Fritze - v. Alvensleben U., Fricke K. J., 2002, A\&A, 392, 1 
Schweizer F., Seitzer P., 1998, AJ, 116, 2206

Sivan J.-P., Stasinska G., Lequeux J., 1986, A\&A, 158, 279

Smith L. J., Norris R. P. F., Crowther P. A., 2002, MNRAS, 337, 1309

Stasińska G., 1984, A\&AS, 55, 15

Vacca W. D., Garmany C. D., Shull J. M., 1996, ApJ, 460, 914

Weilbacher P. M., Duc P.-A., Fritze - v. Alvensleben U., Martin P., Fricke K. J., 2000, A\&A, 358, 819

Zackrisson E., Bergvall N., Olofsson K., Siebert A., 2001, A\&A, 375, 814 



\section{Chapter 3}

\section{Analysing observed star cluster SEDs with evolutionary synthesis models: Systematic uncertainties $^{1}$}

\subsection{Introduction}

Since the seminal work by Tinsley (1968), evolutionary synthesis has become a powerful tool for the interpretation of integrated spectrophotometric observations of galaxies and galactic subcomponents, such as star clusters. Several groups introduced their evolutionary synthesis codes, e.g., Bruzual \& Charlot (1993) [B\&C], Leitherer et al. (1999) [STARBurst99], Fioc \& Rocca Volmerange (1997) [PEGASE], Fritze - v. Alvensleben \& Gerhard (1994) [GALEv] (all with regular updates), with various input physics (evolutionary tracks vs. isochrones from various groups, different sets of stellar spectral libraries, extinction laws ...). The codes do not only vary in terms of input physics but also regarding computational implementation, interpolation routines etc. A number of publications deals with the intercomparison of various evolutionary synthesis codes (e.g. Worthey 1994, Charlot et al. 1996). The impact of uncertainties in the various model parameters (such as in the descriptions of overshooting and mass loss, stellar spectral libraries etc.) on the resulting colours is challenged by Yi (2003). These publications find a good general agreement among the various models, and assign acceptable uncertainties to the model results. Yi (2003) points out the importance of a proper choice of filters for observing objects characterised by different age ranges. This is justified by the light being dominated by stars in different evolutionary stages at different times. The age-metallicity degeneracy is a major drawback for accurate age determinations, especially for

\footnotetext{
${ }^{1}$ This chapter was published as Anders, Bissantz, Fritze - v. Alvensleben \& de Grijs, 2004,
} MNRAS, 347, 196 
young ages $\leq 200 \mathrm{Myr}$.

In addition to the choice of the specific evolutionary synthesis model used another important caveat merits discussion here. A common assumption in dealing with evolutionary synthesis is a well-populated stellar initial mass function (IMF), up to the model's upper mass limit. While this is probably a justifiable assumption for galaxy-sized systems (although uncertainties regarding the IMF slope persist), it certainly breaks down at levels of small (open) star clusters and $\mathrm{OB}$ associations, where stars are formed purely stochastically (by consumption of the available amount of gas), and these statistics dominate the observed dispersion in cluster luminosities. A great deal of progress has been achieved already on this topic, in particular by Cerviño and collaborators (e.g. Cerviño et al. 2002, Cerviño \& Valls-Gabaud 2003). The main conclusion is that for systems more massive than $\approx 10^{5} M_{\odot}$ the impact of the stochasticity of the IMF on the results is - in general - low, and the UV continuum is least affected by stochastic dispersions.

The studies referred to before concentrated on the models themselves. When comparing the model results with observations, in order to constrain the cluster parameters age, metallicity, internal extinction, and mass, one does not only need to take into account the model uncertainties, however. The final parameter uncertainties also depend on the observational errors, the choice of passbands used, their number, spectral coverage and individual filter properties, and the analysis algorithm applied to one's data. The most common way of model-observation comparison for astrophysical purposes is the chi-squared minimisation technique, used e.g. for parameter determination of star clusters (e.g. Maoz et al. 2001, de Grijs et al. 2003a,b), determination of star formation histories of galaxies (e.g. Gavazzi et al. 2002), and photometric redshift determination (e.g. Massarotti et al. 2001). Slightly different, but comparable algorithms, like the least-squares method (e.g. Ma et al. 2002) or maximumlikelihood estimation (e.g. Gil de Paz \& Madore 2002, Bik et al. 2003), are used as well. However, see Bissantz \& Munk (2001) for a critical discussion about the applicability of chi-squared versus least-squares criteria.

The aim of the present paper is a systematic evaluation of inherent uncertainties in the analysis of observed star cluster spectral energy distributions (SEDs) using evolutionary synthesis models. We define an SED as an ensemble of (absolute) magnitudes in a given set of (broad-band) passbands. We pay special attention to the most appropriate choice of passbands to improve future observation strategies. We will point out severe pitfalls, such as trends caused by finite observational errors and unjustified a priori assumptions.

\subsection{Model description}

In section 3.2.1 we present the basic properties of our evolutionary synthesis models. Section 3.2.2 is a general description of our cluster SED analysis algorithm, regardless of whether it is used to study the parameters of observed star clusters or of simulated artificial clusters. In section 3.2.3 we present the specific properties of the artificial clusters (clusters for which SEDs are taken directly from our models) used to simulate observed clusters and study the performance of our analysis tool. From section 3.3 onwards only these artificial clusters are used. 


\subsubsection{Input Models}

We use the single stellar population (SSP) models presented in Schulz et al. (2002), with important improvements regarding the treatment of gaseous emission in the early stages of the cluster evolution, as presented in Anders \& Fritze - v. Alvensleben (2003). These models include isochrones from the Padova group including the TP-AGB phase, and model atmosphere spectra from Lejeune et al. (1997; 1998). These extend from $90 \AA$ through $160 \mu \mathrm{m}$ for five different metallicities, $Z=0.0004,0.004,0.008,0.02=Z_{\odot}$ and 0.05 or $[\mathrm{Fe} / \mathrm{H}]=-1.7,-0.7,-0.4,0$ and +0.4 (i.e., matching the metallicities of the Padova isochrones), and gaseous emission (both lines and continuum) due to the ionising flux from young massive stars. The models can be retrieved from http://www.astro.physik.uni-goettingen.de/ galev/panders/SSPModels/

index.html. For a general description of the stellar models see Bertelli et al. (1994) and Girardi et al. (2000), for details about the specific isochrones in our models see Schulz et al. (2002).

All calculations presented here are based on a Salpeter IMF in the mass range of 0.15 to approximately $70 \mathrm{M}_{\odot}\left(0.15\right.$ to approx. $50 \mathrm{M}_{\odot}$ for super-solar metallicity; following from the Padova isochrones). Stellar synthesis models for a Scalo IMF are presented in Schulz et al. (2002) and Anders \& Fritze - v. Alvensleben (2003), and are available from the aforementioned WWW address.

\subsubsection{General description of the analysis algorithm}

In order to analyse observed SEDs of star clusters in terms of the individual cluster's age, metallicity, extinction, and mass we calculate a grid of models for a large range of values for each of these parameters (except mass, which is a simple scaling of the model mass $\left[M_{\text {model }}=1.6 \times 10^{9} \mathrm{M}_{\odot}\right]$ to the absolute observed cluster magnitudes). Input parameters for the analysis are the time evolution of the spectra of the SSP models, and the derived magnitude evolution in the various passbands.

The individual uncertainties contributing to the overall photometric uncertainties are: the observational uncertainties, an estimated model uncertainty of $0.1 \mathrm{mag}$, and an uncertainty of an additional $0.1 \mathrm{mag}$ for passbands bluewards of the $B$ band due to known calibration and model problems in the UV. The total uncertainty is the square-root of the quadratic sum of these individual errors. The observational and model uncertainties are expected to be independent.

Galactic extinction is taken into account by dereddening the observations using the Galactic extinction values from Schlegel et al. (1998).

First, we calculated dust-reddened spectra, using the starburst galaxy extinction law by Calzetti et al. (2000), assuming a foreground screen geometry,

$$
\begin{gathered}
k^{\prime}(\lambda)=2.659 \times(-1.857+1.040 / \lambda)+4.05 \\
\quad \text { for } 0.63 \mu \mathrm{m} \leq \lambda \leq 2.20 \mu \mathrm{m}, \\
k^{\prime}(\lambda)=2.659 \times\left(-2.156+1.509 / \lambda-0.198 / \lambda^{2}+0.011 / \lambda^{3}\right)+4.05 \\
\quad \text { for } 0.09 \mu \mathrm{m} \leq \lambda<0.63 \mu \mathrm{m}
\end{gathered}
$$

with a reddened flux

$$
F_{\text {red }}(\lambda)=F_{0}(\lambda) \times 10^{0.4 \times \mathrm{E}_{s}(B-V) \times k^{\prime}(\lambda)}
$$


and a range of values for the colour excess of the stellar continuum $\mathrm{E}_{s}(B-V)$. Since the gaseous emission is relevant only for a short time and even then not the dominating term, the difference between the colour excess of the stellar continuum and that from nebular gas emission lines (e.g. Calzetti et al. 2000), is neglected.

We emphasise that the Calzetti law is valid only for starburst galaxies, while for "normal" galaxies (i.e., undisturbed and quiescent spiral and elliptical galaxies) it is probably at least marginally incorrect (due to the lower dust content in such galaxies). However, for our systematic uncertainty analysis, the specific shape of the extinction law assumed is of minor importance.

We construct SEDs from these models by folding the spectra with a large number of filter response functions to obtain absolute magnitudes. The parameter resolutions are:

- Age: 4 Myr resolution for ages from $4 \mathrm{Myr}$ - 2.36 Gyr, 20 Myr resolution for ages from 2.36 Gyr - 14 Gyr;

- Extinction: the resolution is $\Delta \mathrm{E}(B-V)=0.05 \mathrm{mag}$, for $\mathrm{E}(B-V)=$ $0.0-1.0$ mag;

- Metallicities: $[\mathrm{Fe} / \mathrm{H}]=-1.7,-0.7,-0.4,0$ and +0.4 , as given by the Padova isochrones;

- Mass: an arbitrary model mass of $M_{\text {model }}=1.6 \times 10^{9} \mathrm{M}_{\odot}$ is used.

When comparing our observed SEDs with the model SEDs we first determine the mass of the cluster by shifting the model SED onto the observed SED.

A number of these model SEDs (for $M_{\text {cluster }}=M_{\text {model }}$ ) are shown in Fig. 3.1 , for the 5 available metallicities and for 5 representative ages used for the artificial clusters considered in this paper (see Section 3.2.3).

Each of the models in our grid is now assigned a certain probability to be the most appropriate one, given by a likelihood estimator of the form $\mathrm{p} \sim \exp \left(-\chi^{2}\right)$, where $\chi^{2}=\sum \frac{\left(m_{\text {obs }}-m_{\text {model }}\right)^{2}}{\sigma^{2}}$, where $m_{\text {obs }}$ and $m_{\text {model }}$ are the observed and the model magnitudes in each band, respectively, and $\sigma_{\text {obs }}$ are the observational uncertainties. The summation is over all filters. Clusters with unusually large "best" $\chi^{2}$ are rejected, since this is an indication of calibration errors, features not included in the models (such as Wolf-Rayet star dominated spectra, objects younger than $4 \mathrm{Myr}$, etc.) or problems due to the limited resolution of the parameters. The cut-off level is set to a total probability $\leq 10^{-20}$, corresponding to a $\chi_{\text {best }}^{2} \geq 46$. The total probability per cluster is then normalised.

Subsequently, the model with the highest probability is chosen as the "bestfit model". Models with decreasing probabilities are summed up until reaching 68.26 per cent total probability ( $=1 \sigma$ confidence interval) to estimate the uncertainties in the best-fitting model. These uncertainties are in fact upper limits, since their determination does not take into account effects like the existence of several solution "islands" for one cluster (such as e.g. the age-metallicity degeneracy, see below), and discretisation in parameter space.

For real observations, several passband combinations (containing at least 4 passbands) were used for the analysis, to minimise the impact of calibration errors and statistical effects. A minimum of 4 passbands is required to determine the 4 free parameters age, metallicity, extinction and mass independently (see also Anders et al. 2003, de Grijs et al. 2003a,b). 

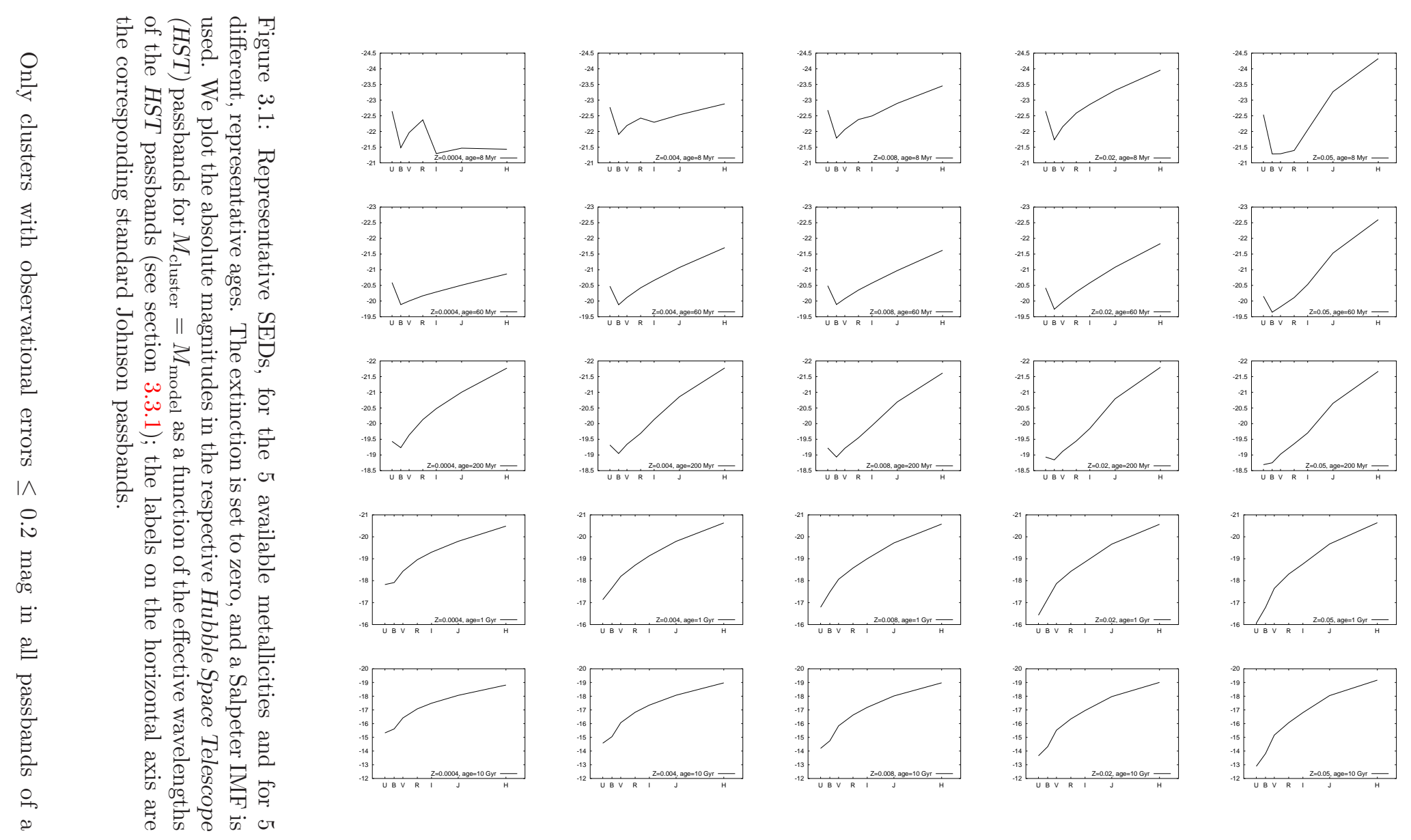
particular combination are included to minimise the uncertainties in the results (except for some artificial clusters considered in this paper, for which we adopt errors $=0.3 \mathrm{mag})$. For each combination, the best-fitting models and their associated parameter uncertainties are determined. For a given cluster all bestfitting models (and the associated uncertainties) originating from the different passband combinations are compared. For each of these best-fitting models the product $\mathrm{P}$ of the relative uncertainties

$$
\mathrm{P}=\frac{\text { age }^{+}}{\text {age }^{-}} \times \frac{\text { mass }^{+}}{\text {mass }^{-}} \times \frac{\text { metallicity } \mathrm{Z}^{+}}{\text {metallicity } \mathrm{Z}^{-}}
$$

is calculated (the superscripts indicate the $1 \sigma$ upper $\left(^{+}\right)$and lower $\left(^{-}\right)$limits, respectively). The relative uncertainty in the extinction is not taken into account, since the lower extinction limit is often zero. The data set with the lowest value of this product is adopted as the most representative set of parameters (with its corresponding parameter uncertainties) for the particular cluster being analysed. In cases where the algorithm converges to a single model, a generic uncertainty of 30 per cent for all parameters is assumed, in linear space, corresponding to an uncertainty of ${ }_{-0.15}^{+0.1}$ dex in logarithmic parameter space. See also Anders et al. (2003) for an application to the star clusters in the dwarf starburst galaxy NGC 1569, and de Grijs et al. (2003a,b) for applications of this algorithm to clusters in the interacting starburst galaxies NGC 3310 and NGC 6745 .

\subsubsection{Artificial clusters}

In this study we will use artificial clusters to investigate the uncertainties related to our analysis on the basis of a comparison with the model grid. The SED magnitudes of the "ideal" artificial clusters are taken directly from the models. Standard parameters of these clusters are: metallicity $[\mathrm{Fe} / \mathrm{H}]=0.0=$ $[\mathrm{Fe} / \mathrm{H}]_{\odot}$, internal extinction $\mathrm{E}(B-V)=0.1$, and ages of 8 Myr ("cluster 1"), 60 Myr ("cluster 2"), 200 Myr ("cluster 3"), 1 Gyr ("cluster 4"), and 10 Gyr ("cluster 5 "). In this standard set only a age variations, and neither metallicity nor extinction variations are considered initially, for reasons of clarity. The impact of varying the metallicity and extinction values is treated separately, see especially Sect. 3.3.3. The cluster mass is the model's mass $1.6 \times 10^{9} \mathrm{M}_{\odot}$, the "observational" errors are set to be $0.1 \mathrm{mag}$ in each filter. Unless otherwise indicated, the clusters in this paper will have these standard parameters.

For each of these 5 sets of artificial cluster parameters 10,000 cluster SEDs were generated by adding statistical noise to the magnitudes of the "ideal" cluster. The errors are drawn from a Gaussian distribution with the Gaussian $\sigma$ corresponding to the "observational" uncertainty ( $=0.1 \mathrm{mag}$ as standard value).

All clusters are analysed separately with our algorithm in order to assess under which conditions and to what accuracy their input parameters are recovered by our method. Subsequently, all clusters originating from a given "ideal" cluster are used to calculate median parameters and their associated uncertainties. The uncertainties are centred around the median solution; they serve as equivalents to the $1 \sigma$ standard deviation around the average values. However, for our analysis we chose to use the median instead of the average of the distribution, since we believe the median to be physically more relevant. We are 
interested in finding the most likely result when comparing our model grid with observations.

Free parameters are the metallicity $[\mathrm{Fe} / \mathrm{H}]$, the extinction $\mathrm{E}(B-V), \log ($ age $)$ and $\log ($ mass $) .[\mathrm{Fe} / \mathrm{H}]$ and $\log ($ age $)$ are used instead of $\mathrm{Z}$ and age because the evolution of magnitudes is approximately linear in $[\mathrm{Fe} / \mathrm{H}]$ and $\log ($ age $)$.

\subsection{Study of the accuracy of our analysis}

\subsubsection{Passbands included in our analysis}

We consider the following filters (the impact of only slightly different filter response curves is small). All filters are taken from the set of available filters for observations of the Hubble Space Telescope (HST)/WFPC2, ACS, and NICMOS cameras.

The standard set of filters is: HST WFPC2 (and ACS) filters F336W (" $U$ "), F439W ("B"), F555W ("V"), F675W ("R"), F814W (" $\Gamma$ "), NICMOS (NIC2 camera) F110W ("J"), F160W (" $H$ "). This standard set will be referred to as "UBVRIJH". In addition the following filters are included in our study as well: the HST WFPC2 (and ACS where appropriate) wide filters F300W ("wide $U$ "), F450W ("wide B"), F606W ("wide V"), F702W ("wide R") and the HST Strömgren filters F336W ("u" 三 "U"), F410M ("v"), F467M ("b"), F547M (" $y$ ").

In this paper we will use the term "UV passband" essentially for the U band, and the term "NIR passbands" for the $\mathrm{J}$ and $\mathrm{H}$ bands.

In the relevant figures, the horizontal lines mark the input values, and the symbols represent the median of the recovered values with the associated uncertainties. The clusters with "cluster number" $=1 \leq \mathrm{x}<2$ are clusters with the youngest input age of $8 \mathrm{Myr}$, clusters with "cluster number" $=2 \leq \mathrm{x}<3$ are clusters with an input age of $60 \mathrm{Myr}$, and so on (this offset is chosen for reasons of clarity).

\subsubsection{Choice of passband combination}

First, we investigate which passbands contain the maximum amount of information, and hence which passbands are preferred for observations, if one can obtain observations in only a limited number of passbands. This aims at improving future observing strategies.

\section{Importance of individual passbands}

In Fig. 3.2 we present the dispersions in our recovered parameters using the standard input parameters, and SEDs covering the full wavelength range UBVRIJH, compared with passband combinations where one of the UBVRIJH passbands is left out.

This figure provides direct evidence of the importance of the $U$ band (and to a lesser degree also of the $B$ band) for all stages of cluster evolution, while for ages $>1$ Gyr also a lack of the $V$ band results in problems to recover the age. The systematic deviations from the input values for the combinations without the $U$ or $B$ bands are caused by an insufficiently accurate determination of the cluster metallicity. The resulting SED changes are therefore balanced by the analysis 

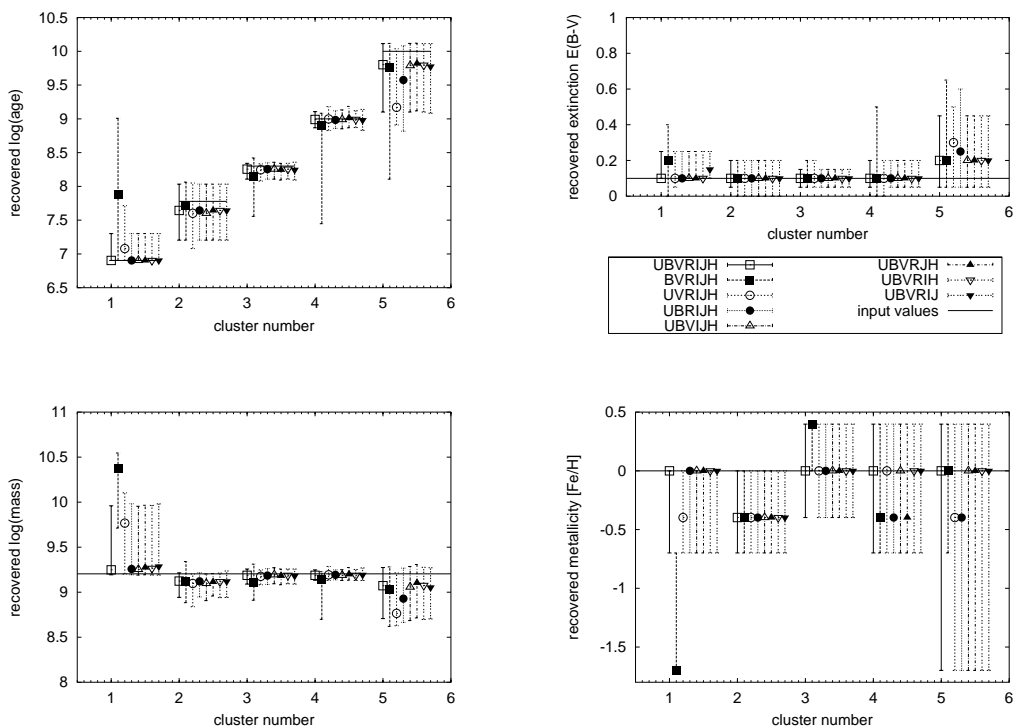

Figure 3.2: Dispersion of recovered properties of artificial clusters, assuming availability of $U B V R I J H$ and passband combinations rejecting one of the $U B$ VRIJH passbands, as indicated in the legend. Cluster parameters are standard.

algorithm by adjusting the extinction and/or age, and are also accompanied by higher-than-input median masses in our fit results.

Systematic biases are only apparent in the age determination of the oldest artificial cluster (with a slight bias towards younger recovered ages), balanced by an overestimate of the internal extinction (which is a sign of the age-extinction degeneracy) and a minor bias towards smaller median masses. For the $60 \mathrm{Myr}-$ old artificial cluster, the metallicity determination leads to an underestimate (presumably due to the criss-crossing of the models and/or the non-negligible impact of the age-metallicity degeneracy at these ages) for all passband combinations, while for the oldest cluster the uncertainty in the metallicity determination encompasses almost the entire available range.

In general, the median values recovered by our code agree fairly well with the input parameters, with the exceptions mentioned above. The parameter dispersions are largest for the young (ages $\leq 60 \mathrm{Myr}$ ) and the oldest (age $=10$ Gyr) clusters. This is caused by the criss-crossing of the models for young ages and the flat magnitude evolution for old ages.

The importance of the $U$ and $B$ band is immediately apparent from the overview of artificial SEDs presented in Fig. 3.1. $U$ and $B$ are important for tracing the hook-like structure for young ages, while there appears to be a kink in the SEDs at the $V$ band for older ages.

\section{Combinations of 4 passbands}

The minimum number of passbands required to determine the 4 free parameters age, metallicity, extinction and mass independently is four.

In Figs. 3.3 and 3.4 we present the recovered parameters for UBVRIJH compared with various passband combinations consisting of 4 passbands, for optical filters only and including one near-infrared (NIR) band, respectively. 

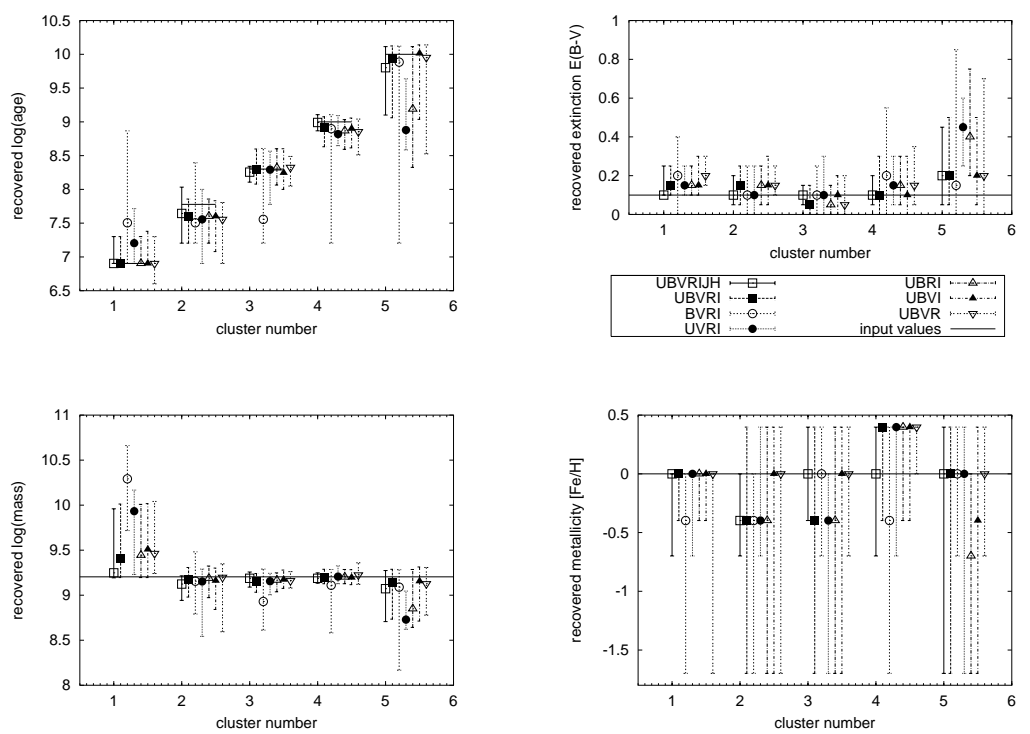

Figure 3.3: Dispersion of recovered properties of artificial clusters, assuming availability of various optical passband combinations, as indicated in the legend. Cluster parameters are standard.
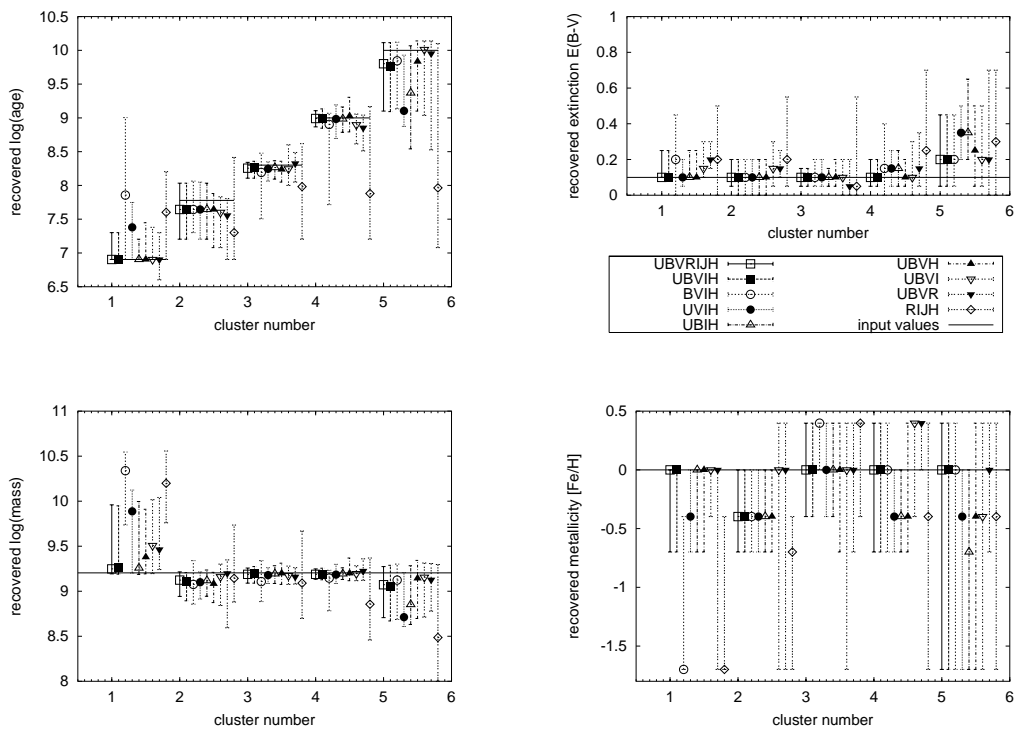

Figure 3.4: Dispersion of recovered properties of artificial clusters, assuming availability of various optical+NIR passband combinations, as indicated in the legend. Cluster parameters are standard.

For optical passbands only, the $U$ band plays a major role once more, especially in determining the metallicity. Missing $U$-band information leads to underestimates of the metallicity, thereby causing extinction values and ages to be adjusted improperly, and hence this also leads to incorrect mass estimates. 

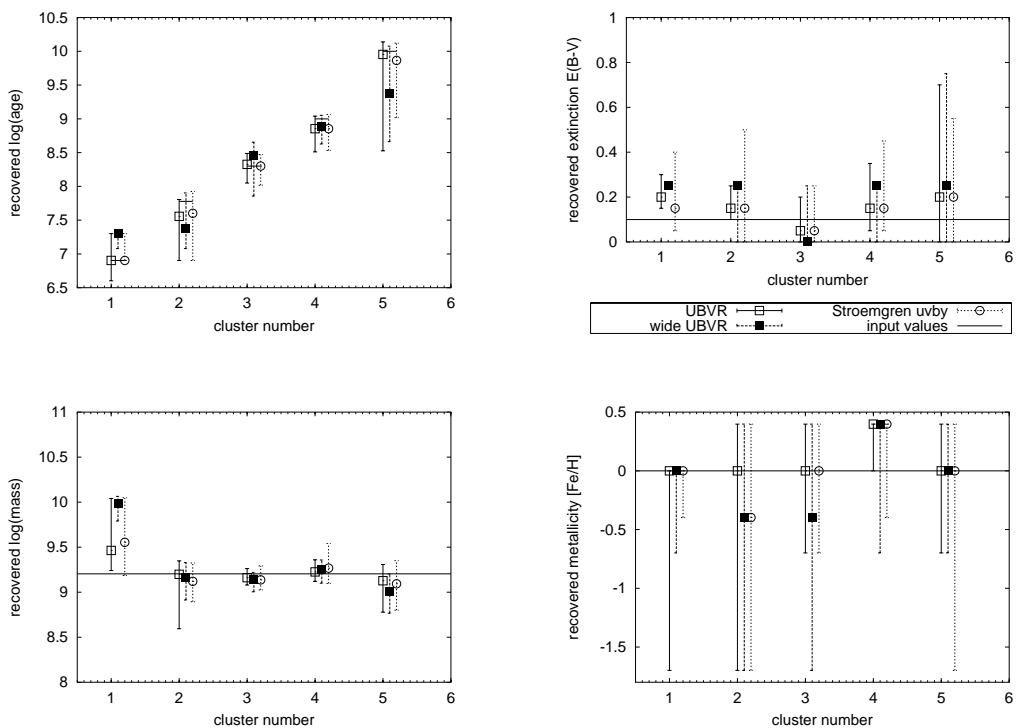

Figure 3.5: Dispersion of recovered properties of artificial clusters, comparing various wide and medium-band HST filters, as indicated in the legend. Cluster parameters are standard.

Even in cases where the median is recovered correctly, such clusters show the largest uncertainties. In some cases, missing $B$-band information has similar effects, especially for the youngest cluster, while for the oldest cluster the $B$ band is vital to break the age-extinction degeneracy. Only for the oldest cluster, the $V$ band contains vital information, which is in accordance with our results in Sect. 3.3.2.

For optical+NIR passbands, the situation is similar: The $U$ band (and to a lesser degree also the $B$ band) is essential. Generally, the offsets from the input values and the uncertainty ranges are smaller than for optical passbands only, thus proving the importance of NIR data. Choosing a NIR band closely resembling the $K$ band instead of $J$ or $H$ would give similar results, possibly restricting the values slightly better. However, we concentrated on the $H$ band since there are more observations available in $H$ in the HST data archive than for filters with longer central wavelengths.

In Fig. 3.4 we also see the effect of a limited wavelength coverage: in all parameters, the RIJH combination gives the worst results (see also de Grijs et al. 2003a). Similar, but less pronounced is the effect for the UBVR combination.

Fig. 3.5 compares the normal WFPC2 UBVR system with the corresponding passband combination using the WFPC2 wide filters. In addition, results based on the medium band Strömgren filter system of WFPC2 are shown.

In most cases the wide filter system gives slightly worse results than the standard system. However, driven by the wider filter response curves and the associated smaller observational errors thanks to the larger flux throughput, the wide system might be preferable, e.g., for faint objects.

Using the WFPC2 Strömgren medium-band system does not result in significant improvements compared to wide-band systems. In conjunction with the lower flux throughput (caused by the narrower bandwidth) this system seems 
less preferable for our purpose. We emphasise that this only holds for our SED analysis.

In de Grijs et al. (2003a) we investigated the impact of the choice of passbands for the young cluster system (with ages of few $\times 10-100 \mathrm{Myr}$ ) in NGC 3310 with HST data from the UV through to the NIR. Starting with the full set of available passbands, we studied the changes in accuracy of the recovered parameters if we repeated the analysis using only a subset of our passbands. By comparing the results from our analyses using all passbands with those from smaller subsets we found severe biases in the age distributions originating from different passband combinations, in particular for combinations biased towards longer wavelengths (VIJH), but also for $U V-U B V$ (covering shorter wavelengths only) and BVIJH, consistent with the results presented here.

\section{Conclusions on the choice of passbands}

From these comparisons we conclude that the passband combinations for the most reliable parameter determination must include the $U$ band, the $B$ band, and use the maximum available wavelength range, preferably including at least one NIR band. If only observations in 4 passbands can be obtained, the best combinations are $U B I H$ or $U B V H$, especially for genuinely old objects, and UBVI, if NIR data cannot be acquired. We emphasise once again that tracing the kink around the $B / V$ band in the SEDs (see Fig. 3.1) is vital. For improved metallicity determinations, and consequently for improved determinations of the other parameters as well, NIR data seem to be crucial (for young clusters the $U / B$ bands are also important, in order to determine the metallicity correctly). However, due to the limited metallicity resolution (and the numerous effects the metallicity has on the synthetic magnitudes), the metallicity determination remains the weakest point in our cluster analysis algorithm, and presumably in any routine using synthetic magnitudes from stellar isochrones or tracks.

\subsubsection{Varying the input parameters}

In this section we investigate to which extent the input parameters can be recovered as a function of their respective values and observational errors.

\section{Using all 7 filters}

Fig. 3.6 shows, for a range of observational uncertainties, the reliability of our recovered parameters if the standard set of filters (UBVRIJH) is available. We caution that we still apply the model uncertainty of 0.1 mag (and an additional uncertainty of 0.1 mag for UV passbands).

A slight trend towards an underestimate of the ages, balanced by a slight overestimate of the internal extinction and an occasional underestimate of the metallicity, is seen. However, even for the largest observational errors of 0.3 mag that we tested for, all recovered parameters are consistent with the input parameters, within the uncertainties.

With increasing observational errors, there seems to be a trend to underestimate the ages for the oldest cluster, balanced by an increasing overestimate of the internal extinction. For genuinely old cluster systems, this degeneracy can be broken by restricting the extinction range. This is generally justified, since such systems are usually dust-poor, if not dust-free, and show fairly homogeneous extinction distributions. 

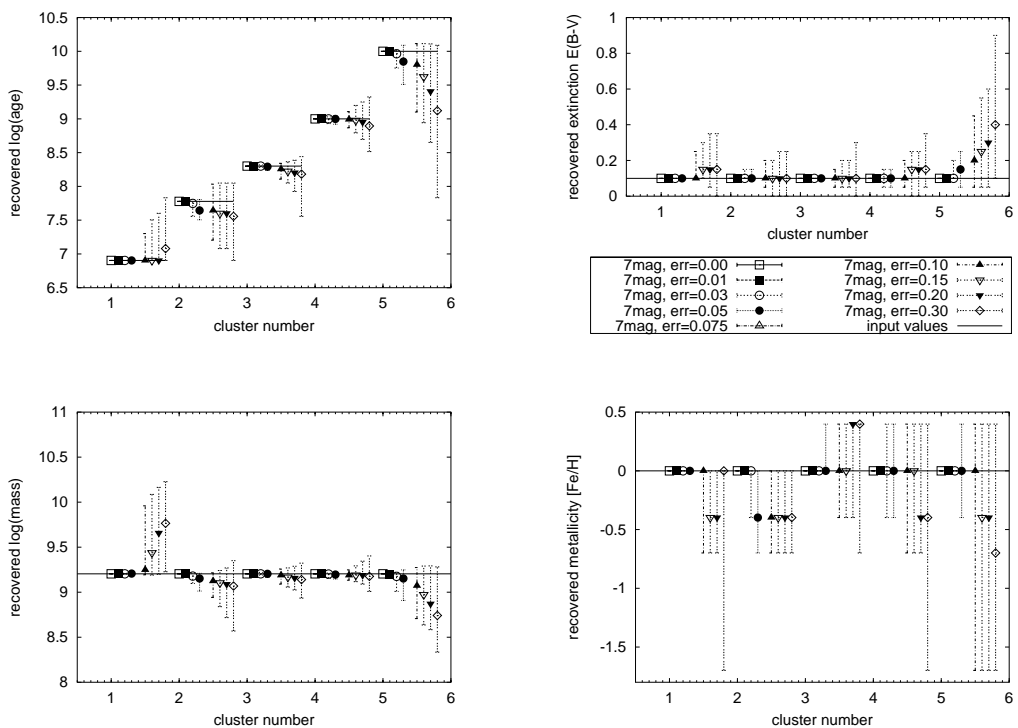

Figure 3.6: Dispersion of recovered properties of artificial clusters, assuming availability of UBVRIJH magnitudes and varying observational errors, as indicated in the legend. Other parameters are standard.
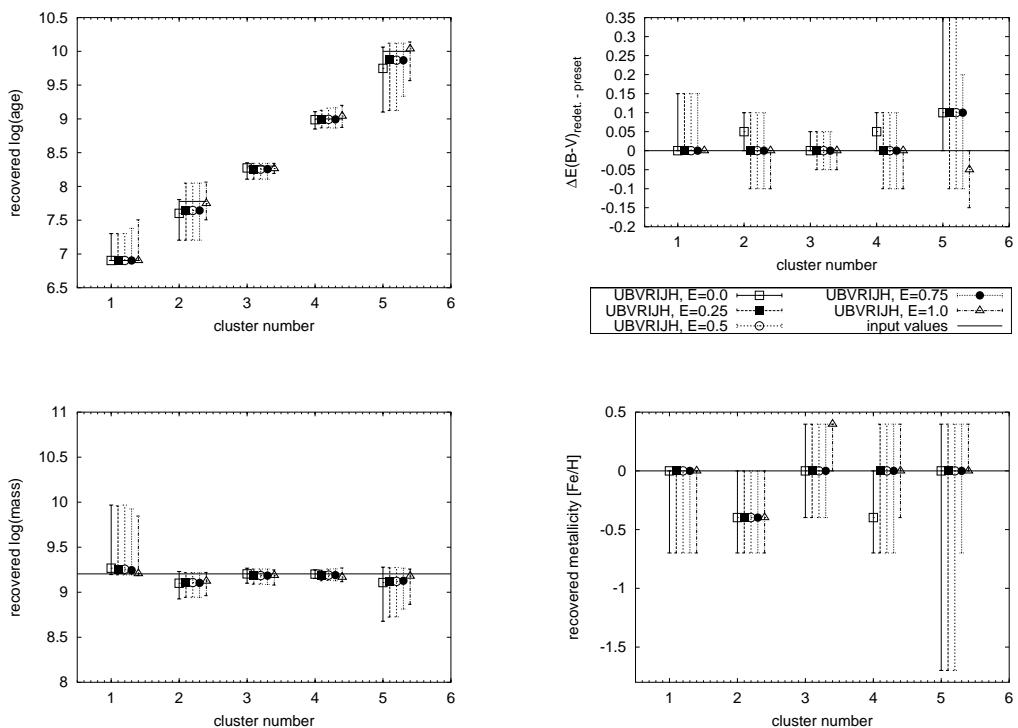

Figure 3.7: Dispersion of recovered properties of artificial clusters, assuming availability of UBVRIJH magnitudes and varying internal extinction values, as indicated in the legend. Other parameters are standard.

Fig. 3.7 shows that the degree to which our code recovers the input parameters is largely independent of the input extinction value, with the exception of the ages recovered for the oldest artificial clusters (in this latter case clear signs of the age-extinction degeneracy are apparent). The remaining deviations of 

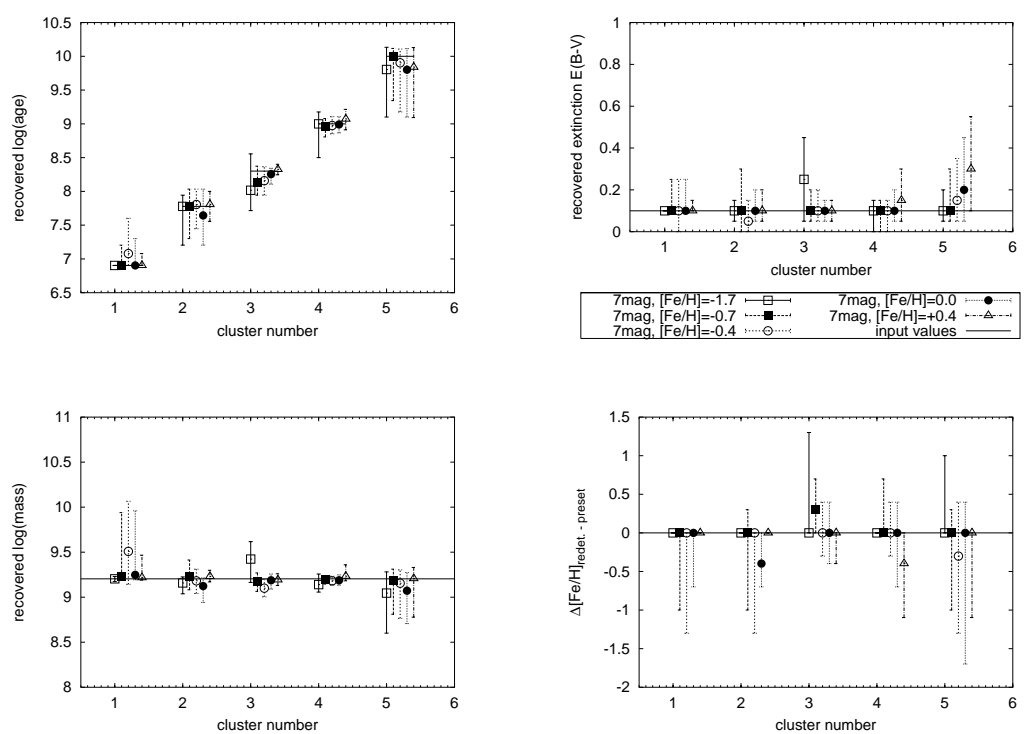

Figure 3.8: Dispersion of recovered properties of artificial clusters, assuming availability of UBVRIJH magnitudes and varying metallicity values, as indicated in the legend. Other parameters are standard.

the median recovered values from the input values are always less than 0.2 dex, and in most cases even smaller. The deviations in metallicity and extinction are one step in resolution (except for the extinction of the oldest cluster, which is, in most cases, 2 steps off). Small trends for increasing age underestimates with lower input extinction are discernible.

Fig. 3.8 indicates good agreement between the input parameters and their recovered values for all 5 metallicities. Median extinction values and metallicities match the input values very well. The age determination is correct to $\Delta \log$ (age) $\lesssim 0.25$ dex. The mass is recovered very well, as is the extinction. The various metallicity input values are in general correctly recovered, only in few cases a difference of one resolution step is seen.

\section{Using the minimum of 4 filters}

The following figures show the accuracy if observations in only the minimum of 4 passbands are available (i.e., a more realistic case). We discuss the bestsuited 4-passband-combination identified in Sect. 3.3.2, including the $H$ band, i.e. the combination $U B I H$.

Fig. 3.9 shows significant trends caused by increasing observational errors, especially for the oldest clusters. For the other clusters, the trends are less severe, with deviations of less than a factor of 2 , or one step in the metallicity resolution. For the oldest cluster, the deviations are up to 1 dex in age, 2 steps in metallicity, 0.35 mag in $\mathrm{E}(B-V)$ and 0.5 dex in mass, for the largest observational errors, i.e. 0.3 mag.

Fig. 3.10 shows the recovered values for $U B I H$ and various input extinction values. For all but the oldest cluster, the recovered parameters reproduce the input values very well. The offsets and uncertainties are slightly larger, but comparable to the corresponding values for UBVRIJH. 

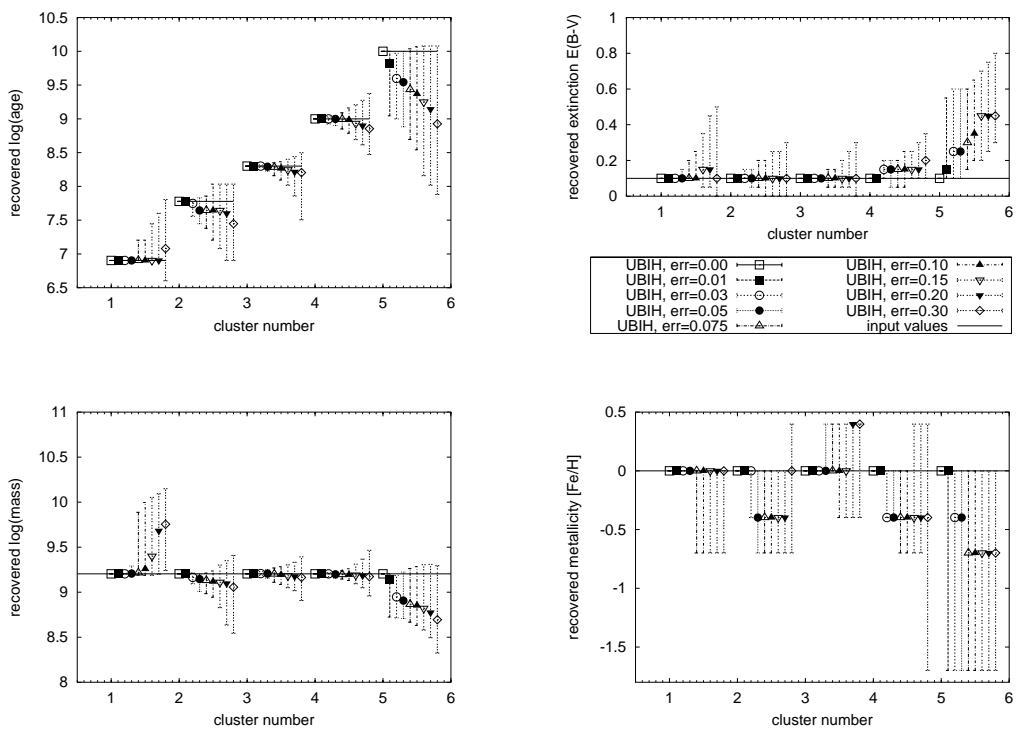

Figure 3.9: Dispersion of recovered properties of artificial clusters, assuming availability of $U B I H$ magnitudes and varying observational errors, as indicated in the legend. Other parameters are standard.
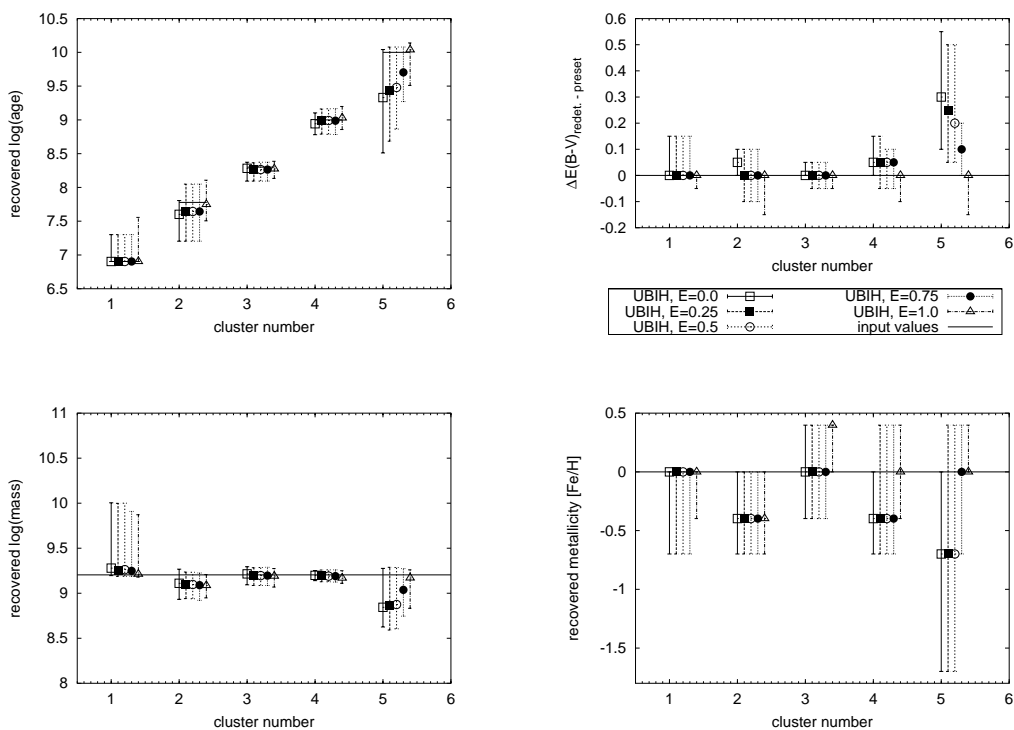

Figure 3.10: Dispersion of recovered properties of artificial clusters, assuming availability of $U B I H$ magnitudes and varying internal extinction values, as indicated in the legend. Other parameters are standard.

For the oldest cluster there are pronounced trends: with increasing input extinction, the recovered age, metallicity and mass estimates increase, while the offsets of the recovered extinction values from their input values decrease. We find that the higher the input extinction, the better all input parameters are 

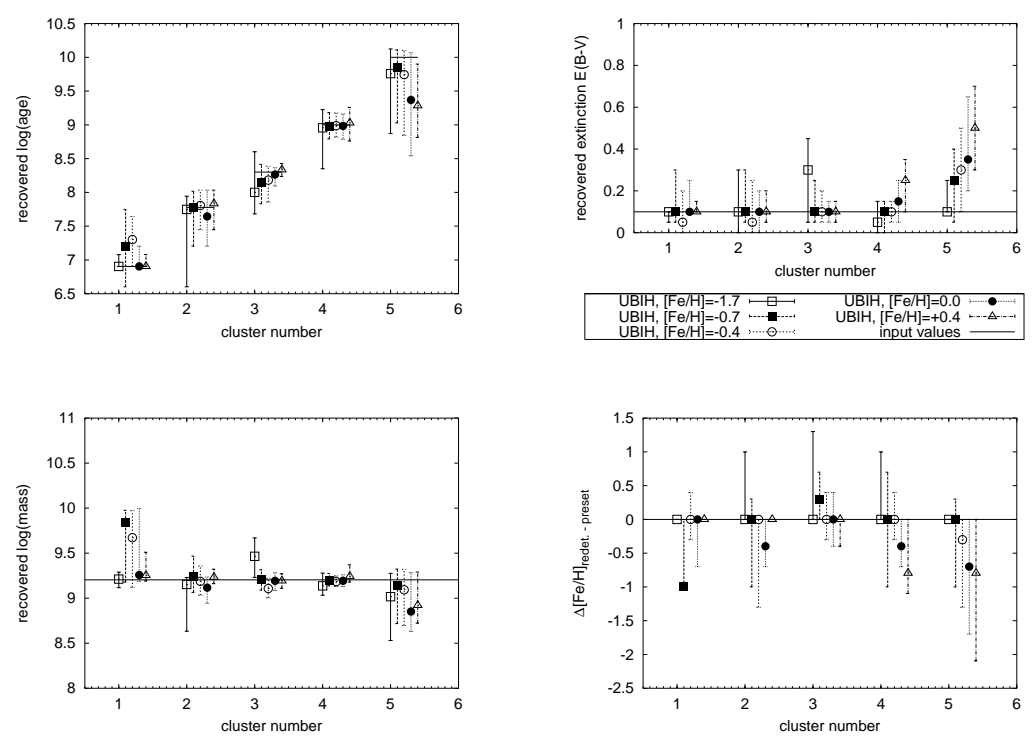

Figure 3.11: Dispersion of recovered properties of artificial clusters, assuming availability of $U B I H$ magnitudes and varying metallicity values, as indicated in the legend. Other parameters are standard.

recovered.

Fig. 3.11 shows the recovered values for $U B I H$ and various input metallicities. The trends with increasing input metallicity are less obvious, except again for the oldest cluster (more significant metallicity underestimates and extinction overestimates with increasing input metallicity). This behaviour is also present, but less pronounced for the second oldest (=1 Gyr old) cluster. For the other, younger clusters, the behaviour appears almost random. This is caused by the slow and steady evolution of magnitudes at large ages: with increasing metallicity the magnitudes become fainter and the colours redder. For younger ages the evolution of the magnitudes is less linear, and they criss-cross several times.

\section{Conclusions on the impact of varying the input parameters}

We have investigated the impact of varying the input parameters on the accuracy of our parameter recovery. We find very good agreement, with generally small deviations, by either varying the input extinction or the input metallicity. Only for the oldest artificial clusters there are significant trends in the recovered values with increasing input extinction and metallicity. This can be attributed to a number of degeneracies. We remind the reader that the upper age limit of the evolutionary synthesis models is 14 Gyr.

For increasing observational uncertainties there are clear trends of increasing recovered extinction and decreasing ages (with a small increase for the youngest cluster only), mass (an increase for the youngest cluster only) and metallicity. The uncertainties increase as well, as expected.

The results using either UBVRIJH or $U B I H$ are fairly similar. Using 4 passbands only slightly increases the offsets of the median recovered values from the input values, as well as the uncertainties. Some trends, especially for the oldest cluster, become more significant. 

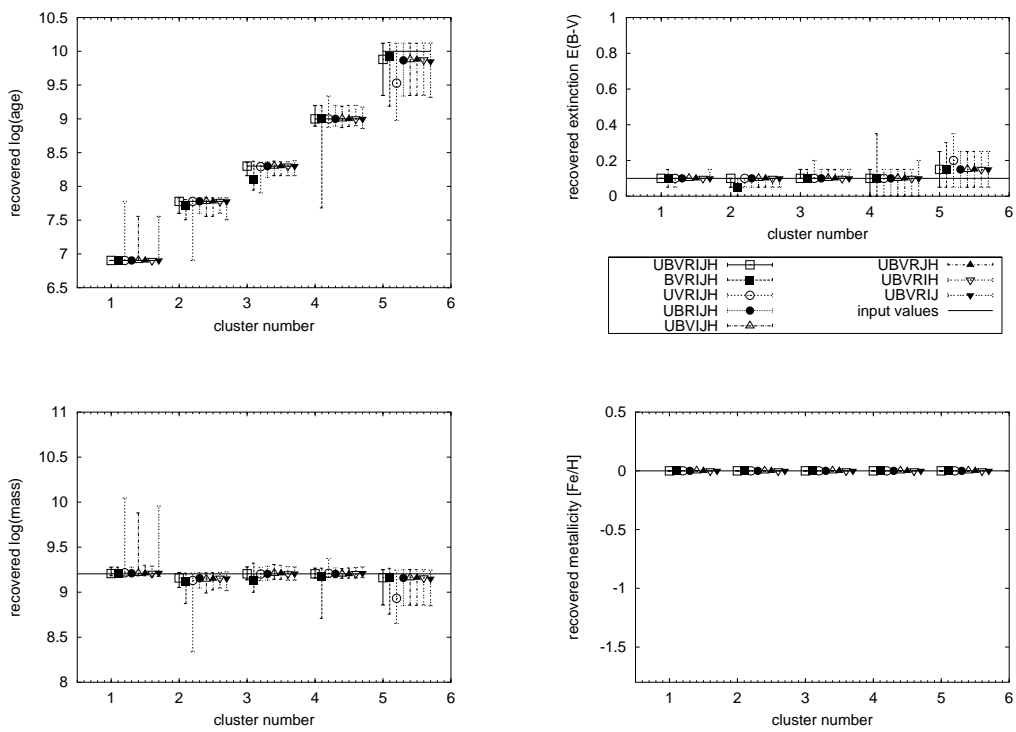

Figure 3.12: Dispersion of recovered properties of artificial clusters, assuming availability of UBVRIJH and passband combinations without one of the UBVRIJH passbands, as indicated in the legend. Solutions were sought with metallicity fixed to the input value. Cluster parameters are standard.

\subsubsection{Restricting the parameter space to the correct ranges}

In this section we investigate the consequences of a priori restrictions of the parameter space. This might make sense in cases where, e.g., large observational errors may inhibit reasonable parameter constraints or where additional information is available, such as spectroscopic abundances, dynamical age estimates for the starburst event that induced the cluster formation, a low-metallicity dwarf galaxy environment, etc. Here, we explore various cases where we restrict some of our free parameters to the (correct) range of input values, and use passband combinations UBVRIJH and combinations lacking one of the UBVRIJH passbands to recover the input parameters.

Fig. 3.12 shows the results when we restrict the metallicity to the (input) solar metallicity. Apart from the oldest cluster (which shows a slight underestimate of the age, balanced by a slight overestimate of the extinction) the recovered values agree almost perfectly with the input values, in any case much better than without metallicity restriction (cf. Fig. 3.2). The importance of the $U$ and $B$ bands, and of the largest possible wavelength coverage are still reflected by the larger uncertainties for observations lacking those filters. The deviations for the oldest cluster are a result of the age-extinction degeneracy.

In Fig. 3.13 we investigate the consequences of restricting the analysis to the input extinction, allowing variations only in metallicity and age. The deviations of the median solutions from the input values, and the uncertainty ranges are significantly reduced compared to the unrestricted case shown in Fig. 3.2. Especially for the oldest cluster, some degeneracies are removed and the recovered values agree much better with the input values than in the unrestricted case. For genuinely old cluster systems the assumption of a generic low extinction 

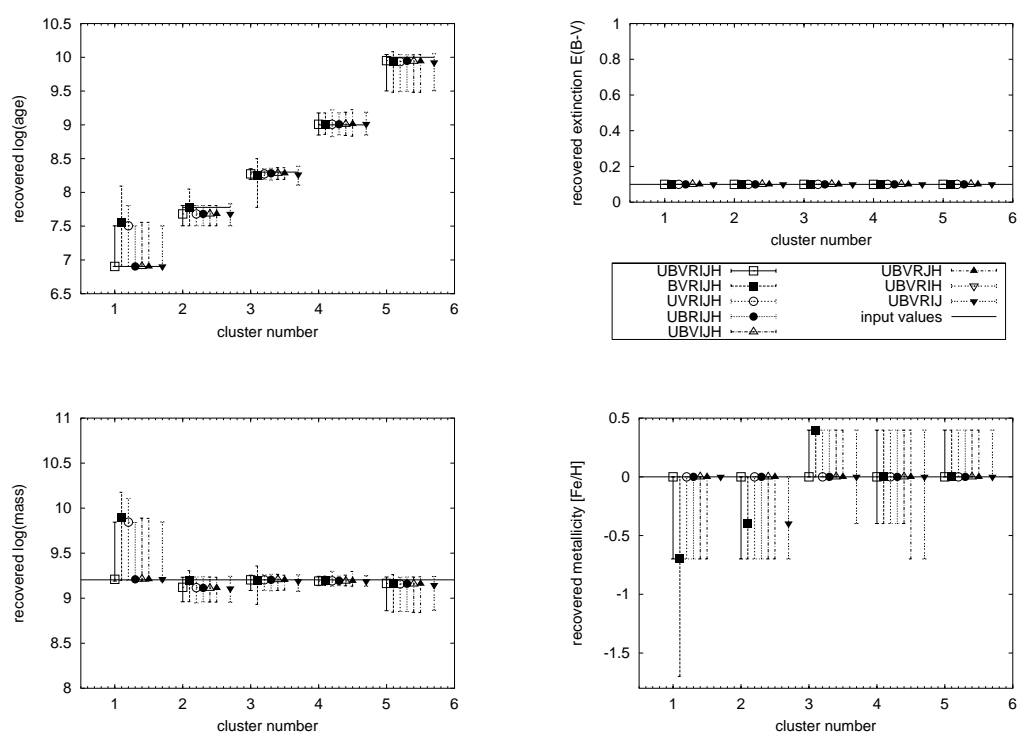

Figure 3.13: Dispersion of recovered properties of artificial clusters, assuming availability of UBVRIJH and passband combinations without one of the UBVRIJH passbands, as indicated in the legend. Solutions were sought with extinction fixed to the input value. Cluster parameters are standard.

may be justified, since such systems are common in old relaxed galaxies with generally low (and uniform) dust content.

Nevertheless, the importance of including the $U$ and $B$ bands is still apparent, especially in the age determination for the youngest cluster. By comparison with Fig. 3.12 we can attribute this behaviour to the age-metallicity degeneracy.

The results from the restriction of both extinction and metallicity to their input values is presented in Fig. 3.14. Clearly, all median values agree perfectly with the values of the remaining input parameters. The few large uncertainty ranges indicate passband combinations that still do not allow to determine the solutions unambiguously, because important information (passbands) are missing. Combinations without these passbands do not allow for a reasonable analysis. This includes combinations without the $U$ or $B$ band (and thus insufficient tracing of the kink in the SEDs) and without the $U$ or $H$ band (thereby restricting the wavelength coverage). The $R / I$ bands seem to be of some importance in the early evolutionary stages to trace the curvature of the SEDs.

Restricting the parameter space of our analysis to the input values for some parameters clearly reproduces the input values of the others, and hence can be used as a sanity check for the reliability of the algorithm. We find the age-extinction degeneracy to be most important for old clusters; for such systems a restriction in the allowed extinction range is usually possible. The agemetallicity degeneracy is responsible for some deviations for clusters younger than 200 Myr. 

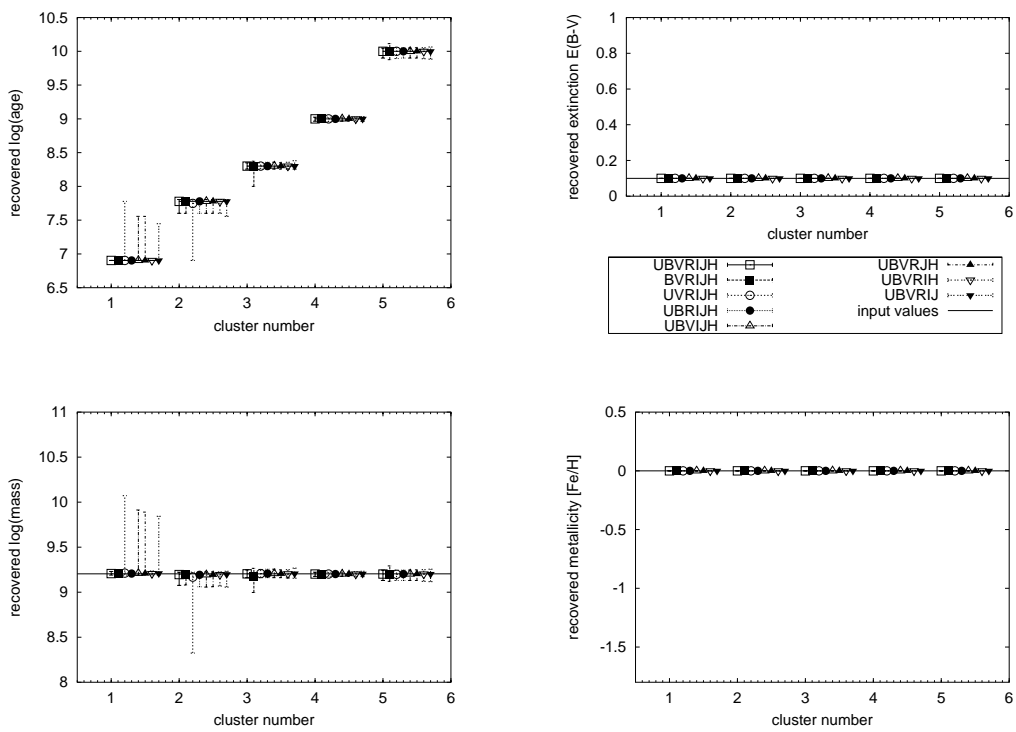

Figure 3.14: Dispersion of recovered properties of artificial clusters, assuming availability of UBVRIJH and passband combinations without one of the UBVRIJH passbands, as indicated in the legend. Solutions were sought with extinction and metallicity fixed to the input values. Cluster parameters are standard.

\subsubsection{Restricting the parameter space to incorrect values}

In this section we investigate the consequences of a priori assuming fixed, but incorrect generic values for the parameters.

First, we investigate the results for various input metallicities, but using solar metallicity to recover the other input parameters (as often done in the literature in studies of interacting and/or merging galaxies). The results are shown in Figs. 3.15 and 3.16. There are significant trends in the age determination in the sense of decreasing ages with decreasing input metallicity. These trends are in some cases accompanied by decreasing extinction. If the actual input metallicity is lower than the fixed metallicity assumed for the analysis, the cluster colours will be too blue for the combined input age and extinction, and for the fixed incorrect metallicity. Hence, either the recovered extinction is driven to lower values and/or the solution to younger ages than their respective input values, since both adjustments result in bluer colours for ages $\geq 200 \mathrm{Myr}$. The results for an actual super-solar input metallicity can be understood the other way around. The youngest clusters show rather randomly distributed recovered values, thereby reflecting the complex behaviour of the magnitudes at such young ages. Applying solar metallicity models (as often seen in the literature) for clusters that intrinsically have sub-solar metallicity results in ages that are too low by up to 60 per cent, masses too low by up to 56 per cent and similarly incorrect extinction values if observations in 7 passbands are available, and even more if the observations were obtained in only 4 passbands.

An equivalent test was done for our cluster sample in NGC 3310, where we compared the results from assuming a fixed, solar metallicity to leaving it as a free parameter (see de Grijs et al. 2003a). We found significantly different age 

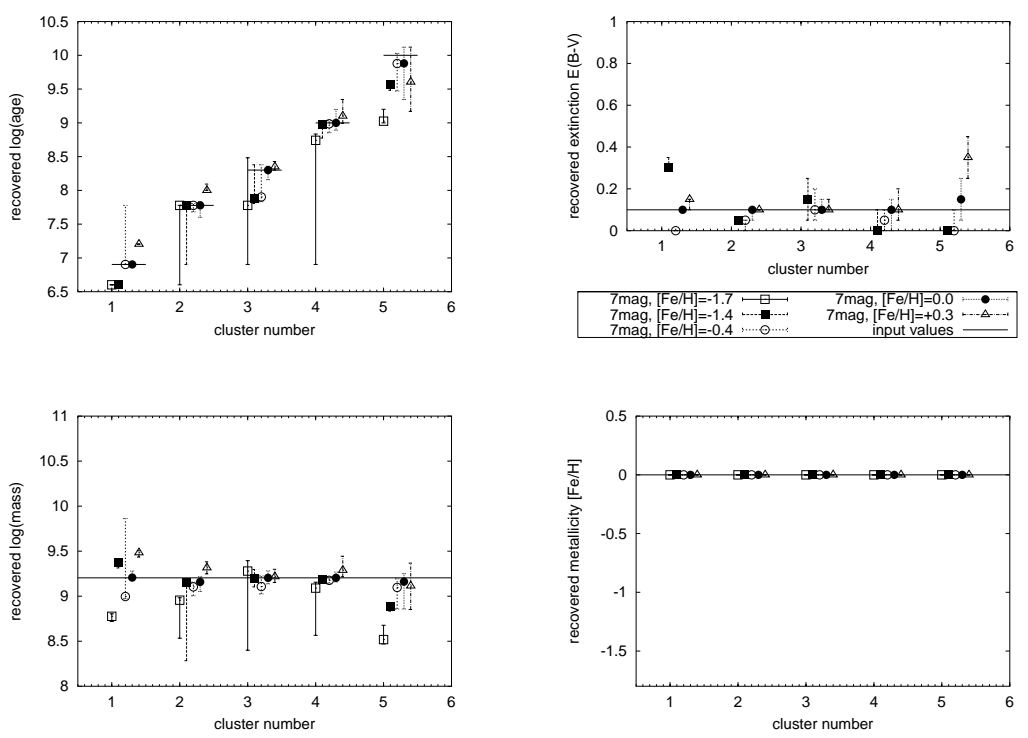

Figure 3.15: Dispersion of recovered properties of artificial clusters, assuming availability of UBVRIJH and various input metallicities, as indicated in the legend. Solutions were sought with metallicity fixed to solar metallicity. Cluster parameters are standard.
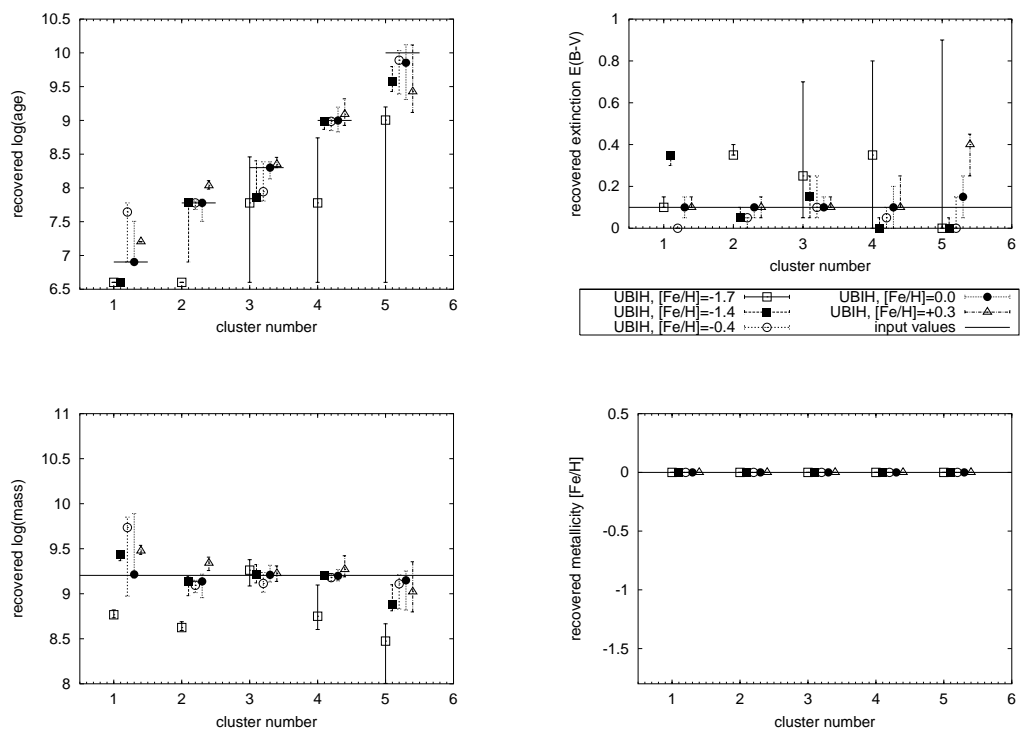

Figure 3.16: Dispersion of recovered properties of artificial clusters, assuming availability of $U B I H$ and various input metallicities, as indicated in the legend. Solutions were sought with metallicity fixed to solar metallicity. Cluster parameters are standard.

distributions in either case, with the analysis in which the metallicity was left as a free parameter resulting in more realistic results in the context of what is 

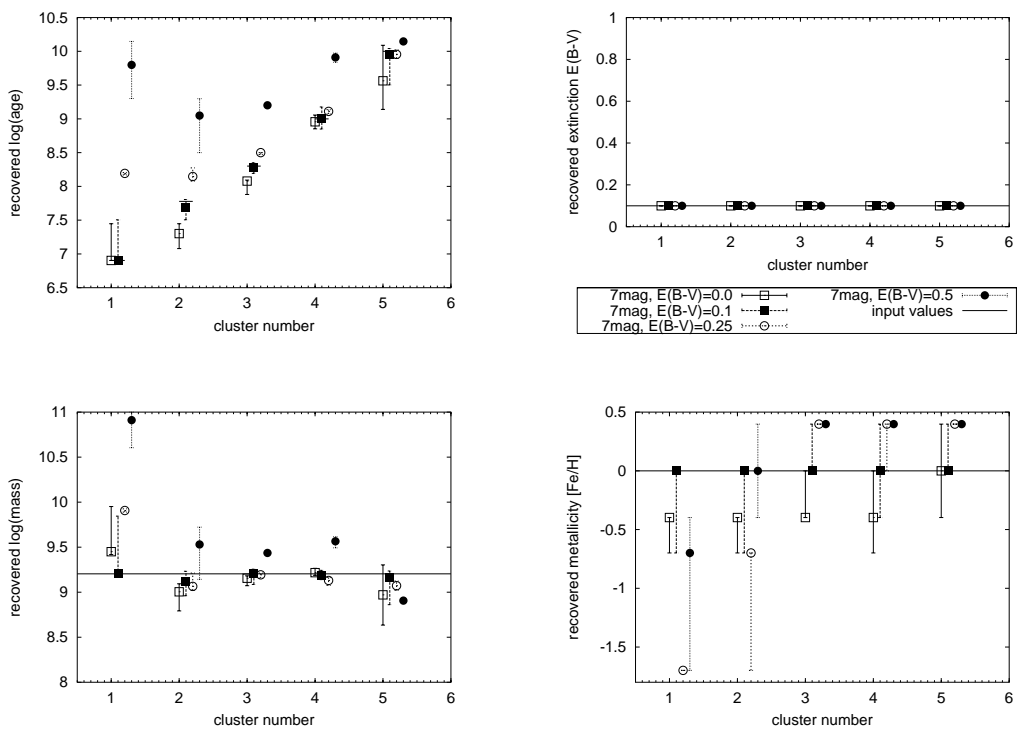

Figure 3.17: Dispersion of recovered properties of artificial clusters, assuming availability of $U B V R I J H$ and various input extinction values, as indicated in the legend. Solutions were sought with extinction fixed to $E(B-V)=0.1$. Cluster parameters are standard.

known about the starburst in NGC 3310 in general. However, since all clusters are young in this cluster system (with ages of a few $\times 10-100 \mathrm{Myr}$ ), this test was limited to young ages.

In de Grijs et al. (2003b) we concentrated on the impact of restricting the allowed metallicity range for the analysis. By assuming a generic, fixed subsolar metallicity (confirmed by spectroscopy), we found that the derived age distribution is fairly robust compared to the case where the metallicity is left as a free parameter, but the peak of the age distribution is significantly broadened. Hence, there is a larger dispersion for individual clusters. This is presumably caused by clusters that do not have the generic metallicity value.

In Figs. 3.17 and 3.18 we show the results for UBVRIJH and UBIH, respectively, if clusters with various extinction values are analysed using a fixed value of $\mathrm{E}(B-V)$. Shown are the results for clusters with input values $\mathrm{E}(B-V)=$ $0.0,0.1,0.25$, and 0.5 , but analysed assuming a fixed extinction $\mathrm{E}(B-V)_{\text {fixed }}=$ 0.1. Considerable changes are observed in the resulting metallicities and ages. In many cases, the deviations from the input values are much larger than the derived uncertainties. For clusters with ages $\gtrsim 200$ Myr there are significant trends of increasing recovered ages and metallicities with increasing input extinction. If the actual input extinction is smaller than the extinction fixed for the analysis, the cluster will be too blue for the combination of input age and metallicity, and for the fixed, incorrect extinction. Hence, either the recovered metallicity is driven to lower values and/or the age must be younger than the corresponding input values, since both result in bluer colours. The results for an actual input extinction higher than the fixed value can be understood the other way around. The youngest clusters show less obvious trends in the distributed recovered values with increasing input extinction, showing the complex 

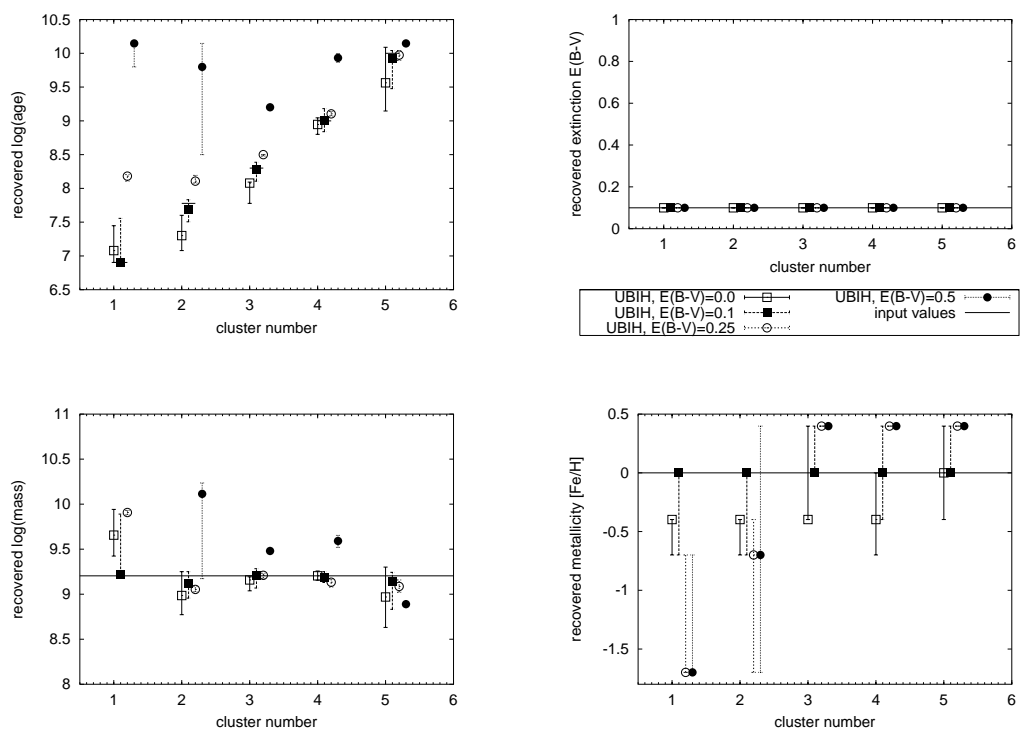

Figure 3.18: Dispersion of recovered properties of artificial clusters, assuming availability of $U B I H$ and various input extinction values, as indicated in the legend. Solutions were sought with extinction fixed to $E(B-V)=0.1$. Cluster parameters are standard.

magnitude behaviour for young ages.

The results from this section again prove the importance of determining the physical parameters of the clusters, such as age, metallicity, and internal extinction (and mass as a derived value), independently, and avoiding any generic assumptions, which might not be justified for all clusters within a given star cluster system. This is true in particular for systems where the existence of two distinct cluster populations is already known or suspected, such as in merging galaxies and galaxies with known colour bimodality in their cluster system.

\subsection{Conclusion}

We have presented a detailed study of the reliability and limitations of our new algorithm to analyse observed SEDs of star clusters, based on broad-band imaging observations, by comparing these to a grid of model SEDs from our evolutionary synthesis code GALEV.

We have computed a large grid of star cluster SEDs on the basis of our GALEV models for simple stellar populations including all relevant stellar evolutionary phases for ages $\geq 4 \mathrm{Myr}$. The models also include metallicity-dependent gaseous line and continuum emission shown to be an important contributor to broadband fluxes in early evolutionary stages. Our grid covers ranges in metallicity of $-1.7 \leq[\mathrm{Fe} / \mathrm{H}] \leq+0.4$, in extinction of $0 \leq \mathrm{E}(B-V) \leq 1$, and ages of $4 \mathrm{Myr}$ $\leq$ age $\leq 14$ Gyr. The models produce spectra from which we derive absolute magnitudes, and hence broad-band SEDs, for any given filter system. Here, we present results for HST broad-band filters widely used for observations and 
Table 3.1: Overview of the most important filters and most/least preferable 4 -passband combinations, if NIR data are available

\begin{tabular}{|c|c|c|c|}
\hline Age & $\begin{array}{c}\text { important } \\
\text { filters }\end{array}$ & $\begin{array}{c}\text { preferable } \\
\text { combinations }\end{array}$ & $\begin{array}{c}\text { combinations } \\
\text { to be avoided }\end{array}$ \\
\hline $\begin{array}{c}\leq \text { few Gyr } \\
\geq \text { few Gyr }\end{array}$ & $\begin{array}{c}U, B \\
B, V, U\end{array}$ & $\begin{array}{c}\text { UVIH, UBVH } \\
\text { BVIH, UBVI }\end{array}$ & $\begin{array}{l}\text { BVIH, RIJH } \\
\text { UVIH, UBIH }\end{array}$ \\
\hline
\end{tabular}

analyses of star cluster systems in external galaxies.

Our parameter analysis algorithm compares a given cluster SED (either observed or theoretical, as done in this study) with the model SEDs from our input parameter grid. Each parameter set is assigned a certain probability, based on an "observation-model" comparison using a chi-squared algorithm. The parameter set with the highest probability is adopted as the best model; the range of parameters from sets with the highest probabilities (up to a total probability of 68.26 per cent) determines the $1 \sigma$ uncertainties in the parameters.

We constructed numerous artificial cluster SEDs, and varied each of the input parameters in turn to assess their effects on the robustness of our parameter recovery. For each clean model artificial cluster SED we calculated 10,000 additional clusters, with errors distributed around the input magnitudes in a Gaussian fashion.

We identified useful and less suitable passband combinations, with the aim to aid in the planning of observational campaigns. Although a large number of passbands is always preferable, any realistic programme will more likely be limited to observations in the minimum number of required passbands to successfully reach its goals. In order to successfully disentangle the three free parameters age, metallicity, and internal extinction based on the shape of a broad-band SED, and to determine the mass of a star cluster by simple scaling of the model magnitudes to the observed level, a minimum of four passbands are required. The most/least preferable passband combinations are summarised in Tables 1 and 2 as a function of the expected age of the cluster population. In all cases, tracing the kink (or hook) in the SEDs around the $B$ band (see Fig. 3.1) is of the highest importance. The inclusion of at least one NIR passband significantly improves the results, since NIR wavelengths allow to efficiently restrict the metallicity range. For the youngest clusters, metallicity estimates are determined by the $U$ and/or $B$ bands. The poorest results are obtained if neither UV information nor $B$ band data are available, or if the available wavelength coverage is very short or biased towards blue or red wavelengths (like RIJH).

By analysing artificial clusters, using a variety of input parameters (specifically age, metallicity, and internal extinction) with our new code, we find in general good agreement between the recovered and the input parameters. Only the oldest, 10 Gyr-old artificial clusters show significant signs of the well-known age-metallicity-extinction degeneracy. 
Table 3.2: Overview of the most important filters and most/least preferable 4-passband combinations, if no NIR data are available

\begin{tabular}{|c|c|c|c|}
\hline Age & $\begin{array}{c}\text { important } \\
\text { filters }\end{array}$ & $\begin{array}{c}\text { preferable } \\
\text { combinations }\end{array}$ & $\begin{array}{c}\text { combinations } \\
\text { to be avoided }\end{array}$ \\
\hline$\leq$ few Gyr & $U, B$ & $U B R I, U B V I$ & $B V R I, U V R I$ \\
$\geq$ few Gyr & $B, V, U$ & $U B V I$ & $U V R I, U B R I$ \\
\hline
\end{tabular}

We have considered several a priori restrictions of the parameter space, both to the (correct) input values and to some commonly assumed values. We easily recover all remaining input values correctly if one of them is restricted, a priori to its correct input value; this also provides a sanity check for the reliability of our code. We find the age-extinction degeneracy to be most important for old clusters. For such systems, an a priori restriction of the allowed extinction range is often possible and shown to be very useful. The age-metallicity degeneracy is responsible for some misinterpretations of clusters younger than $200 \mathrm{Myr}$.

If we, however, restrict one or more of our input parameters a priori to incorrect values (such as using, e.g., only solar metallicity, as often found in the literature), large uncertainties result in the remaining parameters. While certain restrictions might be justified in specific cases, we strongly advice caution in more complex cases, such as in interacting galaxies or in galaxies with known colour bimodality in their cluster systems.

Finally, we conclude that reliable determination of physical star cluster parameters is possible on the basis of broad-band imaging, provided the availability of a useful set of observational passbands, containing at least four filters, a sufficiently long wavelength base line, and reasonable photometric accuracy. We show that a small, but suitably chosen filter set with deep observations (and the correspondingly small uncertainties) gives more reliable results than a larger number of shallow exposures in inappropriate or redundant filters.

The method we have developed is a versatile and useful tool for the interpretation of large multi-colour data sets for star clusters of different ages and in a large variety of environments, such as provided by, e.g., our ST-ECF/ESO ASTROVIRTEL ${ }^{2}$ project "The Evolution and Environmental Dependence of Star Cluster Luminosity Functions" (PI R. de Grijs).

\subsection{Acknowledgements}

PA is partially supported by DFG grant Fr 916/11-1.

${ }^{2}$ ASTROVIRTEL is a project funded by the European Commission under $5 \mathrm{FP}$ contract HPRI-CT-1999-00081. 


\section{Bibliography}

Anders P., Fritze - v. Alvensleben U., 2003, A\&A, 401, 1063

Anders P., de Grijs R., Fritze - v. Alvensleben U., Bissantz N., 2004, MNRAS, 347,17

Bertelli G., Bressan A., Chiosi C., Fagotto F., Nasi E., 1994, A\&AS, 106, 275

Bik A., Lamers H. J. G. L. M., Bastian N., Panagia N., Romaniello M., 2003, A\&A, 397, 473

Bissantz N., Munk A., 2001, A\&A, 376, 735

Bruzual G. A., Charlot S., 1993, ApJ, 405, 538

Calzetti D., Armus L., Bohlin R. C., Kinney A. L., Koornneef J., StorchiBergmann T., 2000, ApJ 533, 682

Cerviño M., Valls-Gabaud D., 2003, MNRAS, 338, 481

Cerviño M., Valls-Gabaud D., Luridiana V., Mas-Hesse J. M., 2002, A\&A, 381, 51

Cerviño M., Luridiana V., Castander F. J., 2000, A\&A, 360L, 5

Charlot S., Longhetti M., 2001, MNRAS, 323, 887

Charlot S., Worthey G., Bressan A., 1996, ApJ, 457, 625

Fioc M., Rocca - Volmerange B., 1997, A\&A, 326, 950

Fritze - v. Alvensleben U., Gerhard O. E., 1994, A\&A, 285, 751

Gavazzi G., Bonfanti C., Sanvito G., Boselli A., Scodeggio M., 2002, ApJ, 576, 135

Gil de Paz A., Madore B. F., 2002, AJ, 123, 1864

Girardi L., Bressan A., Bertelli G., Chiosi C., 2000, A\&AS, 141, 371

de Grijs R., Fritze - v. Alvensleben U., Anders P., Gallagher III J. S., Bastian N., Taylor V. A., Windhorst R. A., 2003a, MNRAS, 342, 259

de Grijs R., Anders P., Bastian N., Lynds R., Lamers H.J.G.L.M., O’Neil Jr. E.J., 2003b, MNRAS, 343, 1285 
Leitherer C., Schaerer D., Goldader J. D., Delgado R. M. G., Robert C., Kune D. F., de Mello D. F., Devost D., Heckman T. M., 1999, ApJS, 123, 3

Lejeune T., Cuisinier F., Buser R., 1997, A\&AS, 125, 229

Lejeune T., Cuisinier F., Buser R., 1998, A\&AS, 130, 65

Ma J., Zhou X., Chen J., Wu H., Jiang Z., Xue S., Zhu J., 2002, A\&A, 385, 404

Maoz D., Barth A. J., Ho L. C., Sternberg A., Filippenko A. V., 2001, AJ, 121, 3048

Massarotti M., Iovino A., Buzzoni A., Valls-Gabaud D., 2001, A\&A, 380, 425

Moy E., Rocca - Volmerange B., Fioc M., 2001, A\&A, 365, 347

Schlegel D. J., Finkbeiner D. P., Davis M., 1998, ApJ, 500, 525

Schulz J., Fritze - v. Alvensleben U., Möller C. S., Fricke K. J., 2002, A\&A, 392, 1

Tinsley B. M., 1968, ApJ, 151, 547

Worthey G., 1994, ApJS, 95, 107

Yi S., 2003, ApJ, 582, 202 



\section{Chapter 4}

\section{Star Cluster Formation and Evolution in the Dwarf Starburst Galaxy NGC $1569^{1}$}

\subsection{Introduction}

The dwarf starburst galaxy NGC 1569 (Arp 210, VII Zw 16, UGC 03056) has attracted attention for almost 30 years, starting with the observations by Hodge (1974) and de Vaucouleurs et al. (1974). Huge filamentary features are seen in the outskirts of the galaxy, like the so-called " $\mathrm{H} \alpha$ arm", as well as bubbles and super-bubbles in all parts of the galaxy's main body (e.g. Waller 1991, Heckman et al. 1995). This bubble structure has a complicated velocity structure (Tomita et al. 1994) and is accompanied by signs of galactic superwinds and outflows (Heckman et al. 1995, Della Ceca 1996), caused by the massive energy input from collective supernova (SN) explosions associated with the starburst. Whether the superwinds are strong enough to remove a significant amount of material from the gravitational potential of NGC 1569 is still being debated (this would predominantly remove the high-metallicity SN ejecta, see e.g. Della Ceca 1996, Martin et al. 2002). Signs of recent star formation are seen along the bubble walls, which is thought to be strong evidence for stochastic self-propagating star formation (e.g., Gerola \& Seiden 1978, Seiden et al. 1984).

The properties of the two "super star clusters" (SSCs), usually called "A" and "B" (nomenclature from Arp \& Sandage 1985), have been studied in great detail. First described by Arp \& Sandage (1985), a significant effort was spent on characterising the properties of these clusters. Spectroscopic mass estimates $\left((2.3-8.3) \times 10^{5} M_{\odot}\right)$ were derived by Ho \& Filippenko (1996) and Gilbert \& Graham (2001). Cluster "A" was resolved into a double cluster with different stellar content in each of the components (de Marchi et al. 1997, Buckalew et al. 2000 and Maoz et al. 2001, but see González Delgado et al. 1997 and Hunter et

\footnotetext{
${ }^{1}$ This chapter was published as Anders, de Grijs, Fritze - v. Alvensleben \& Bissantz, 2004, MNRAS, 347, 17
} 
Table 4.1: Overview of the observations of NGC 1569

\begin{tabular}{crccr}
\hline \hline Filter & $\begin{array}{r}\text { Exposure time } \\
(\mathrm{sec})\end{array}$ & Centre $^{a}$ & PID $^{b}$ & $\begin{array}{r}\text { ORIENT }^{c} \\
\left({ }^{\circ}\right)\end{array}$ \\
\hline F336W & $2 \times 400$ & PC & 6423 & -86.172 \\
F380W & $2 \times 60$ & PC & 6111 & 170.066 \\
& $16 \times 600$ & PC & 6111 & 170.066 \\
F439W & $2 \times 40$ & PC & 6111 & 169.883 \\
& $16 \times 700$ & PC & 6111 & 169.883 \\
F555W & $2 \times 20$ & PC & 6111 & 169.714 \\
& $16 \times 500$ & PC & 6111 & 169.714 \\
& $50,2 \times 140$ & PC & 6423 & -86.172 \\
F814W & $2 \times 300$ & PC & 6423 & -86.172 \\
& $50,2 \times 100$ & PC & 6423 & -86.172 \\
F110W & 300 & PC & 6423 & -86.172 \\
F160W & $10 \times 511.95$ & NIC2 & 7881 & -139.691 \\
& $10 \times 511.95$ & NIC2 & 7881 & -139.691 \\
\hline
\end{tabular}

Notes: ${ }^{a}$ - Location of the galactic centre; ${ }^{b}-$ HST programme identifier; ${ }^{c}$ - Orientation of the images (taken from the image header), measured North through East with respect to the V3 axis (i.e., the $\mathrm{X}=\mathrm{Y}$ diagonal of the $\mathrm{WF} 3 \mathrm{CCD}=+180^{\circ}$ ).

al. 2000 ["H00"]). To date the age estimates of various groups agree fairly well, suggesting an age of cluster "A" of around $7 \mathrm{Myr}$ (with probably a small age difference between the two subclusters) and of around 10-20 Myr for cluster "B" (H00, Origlia et al. 2001, Maoz et al. 2001).

However, our knowledge of the remaining clusters is very limited. Only H00 have investigated a larger sample of star clusters in NGC 1569, but concentrate their parameter studies on the SSCs. Hence only comparisons for photometric performance can be made for the other clusters. Only the age of cluster "no. 30 " (age $\approx 30 \mathrm{Myr}$ ) is presented elsewhere (Origlia et al. 2001, nomenclature from $\mathrm{H} 00$ ).

This paper is part of an ongoing study, in which we will evaluate the impact of the environment on the star cluster populations of galaxies with ongoing or recent star cluster formation. While NGC 1569 is a gas-rich starburst dwarf galaxy (Israel 1988), other environments, such as interacting galaxies of various types and at various stages of interaction, will be studied with the same methods in a homogeneous way.

\subsection{Observations and data preparation}

The data were retrieved from the Hubble Space Telescope (HST) data archives, using the ESO/ST-ECF "ASTROVIRTEL" interface, and automatically calibrated using the standard OPUS pipeline (On-the-fly Reprocessing), using the most up-to-date calibration files available. A list of the retrieved WFPC2 and NICMOS data is provided in Table 4.1. The original observations were taken in January 1996, October 1998 and February 1998 for PID 6111, 6423 and 7881, respectively.

Since the retrieved images are from three different proposals, the centerings on the chip, and the orientations vary.

First, we divided the images into groups of the same passband/programme combination. The images were checked for saturation effects, and the groups were subdivided into subgroups with saturation and without saturation of the 
brightest sources.

The images of each subgroup were combined, using the IMALIGN and CRREJECT/COSMICRAYS tasks in IRAF ${ }^{2}$. The subgroups were then rotated, their pixel sizes matched, aligned and trimmed using the appropriate IRAF routines.

The final fields-of-view (FoVs) are: $577 \times 577 \mathrm{PC}$ pixels $\left(26^{\prime \prime} 54 \times 26^{\prime \prime} 54\right)$ for the small FoV (which includes NICMOS coverage), and $777 \times 787 \mathrm{PC}$ pixels $\left(35^{\prime \prime} 74 \times 36^{\prime \prime} 20\right)$ for the larger FoV common only to the WFPC2 observations. These FoVs correspond to areas of $283 \times 283 \mathrm{pc}$ and $381 \times 386 \mathrm{pc}$, respectively, at an adopted distance for NGC 1569 of $2.2 \mathrm{Mpc}$ (see e.g. Israel 1988)

\subsubsection{Source selection}

Objects were selected using a version of DAOFIND (Stetson 1987), running under IDL. Subsequently, the source sizes were estimated by fitting Gaussian profiles to the sources' intensity distributions. "Point-like sources" may contain bright stars in NGC 1569 as well as foreground stars in the Galaxy. At least one obvious foreground star is visible in the lower right-hand corner of Fig. 4.2. Sources with $\sigma \leq 1.7$ pixels (equivalent to $0.83 \mathrm{pc}$ at the distance of NGC 1569) were rejected as being point-like sources. This limit was chosen after an analysis of the distribution of Gaussian $\sigma$ 's, shown in Fig. 4.1. A sum of two Gaussians was fitted to this distribution, one representing point-like sources and the other cluster-like (hence significantly extended) sources. The rejection limit at $\sigma=1.7$ pixels was chosen conservatively to get a clean cluster sample. As pointed out by Whitmore et al. (1999) a broadening of the (intrinsic) point-spread function (PSF) might occur at deep exposures due to jitter and breathing effects. Additional broadening might be caused by crowding of star clusters, although this seems to be a minor issue in NGC 1569 where the star clusters are usually well separated (see Fig. 4.2), and during the alignment and combination of large numbers of single images. These effects are most likely responsible for the pronounced peak around $\sigma \simeq 1.2$ pixels, hence $\approx 0.4$ pixel larger than the corresponding value for a pure WFPC2 PSF. This procedure also removes blends of bright stars and clusters from our sample, for which accurate cluster photometry would be impossible.

We believe that this method gives reliable results, since we are only interested in relative size estimates and we measure the sizes of the clusters in a homogeneous way.

An initial list of cluster candidates was created by cross-correlating the source lists from "saturated" F555W and F814W (comparable to V-band and Iband) images. Subsequently, additional bright sources from the other passbands were added.

Finally, all candidate clusters were examined visually. Spurious detections, remaining cosmic rays covering more than a single pixel and obvious remaining single bright stars or blends of single stars and clusters were rejected in this step.

\footnotetext{
${ }^{2}$ The Image Reduction and Analysis Facility (IRAF) is distributed by the National Optical Astronomy Observatories, which is operated by the Association of Universities for Research in Astronomy, Inc., under cooperative agreement with the (U.S.) National Science Foundation. StsDAs, the Space Telescope Science Data Analysis System, contains tasks complementary to the existing IRAF tasks. We used Version 2.3 (June 2001) for the data reduction performed in this paper.
} 


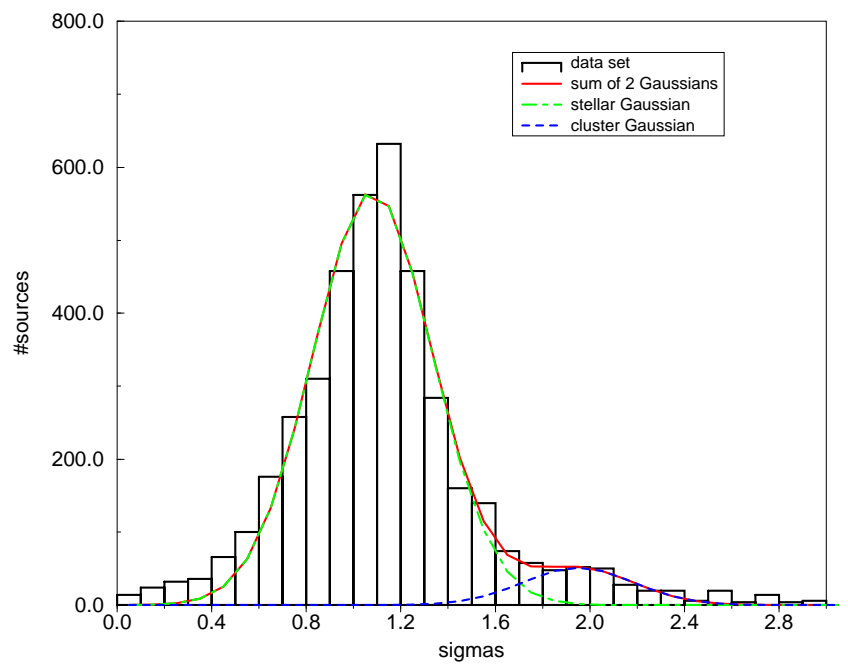

Figure 4.1: Distribution of the Gaussian $\sigma$ 's of the detected sources. Two Gaussians were fitted to this distribution, representing stars and cluster candidates, respectively.

The number of star clusters resulting from this procedure was 168 in the small FoV, and 179 in the larger FoV.

\subsubsection{Photometric calibration}

The coordinates from the source lists obtained in the previous section were used as the centres for aperture photometry, based on DAOPHOT routines adapted for IDL, in all passbands. Standard apertures of 5 pixel source aperture and 5-8 pixel sky annuli were adopted, corresponding to $2.45 \mathrm{pc}$ and $2.45-3.9 \mathrm{pc}$ at the distance to NGC 1569 of $2.2 \mathrm{Mpc}$. Visual inspection allowed us to adjust these standard apertures where necessary (for almost half of the clusters, the apertures and sky annuli needed to be increased), to include a maximum fraction of the source flux and to avoid unrelated features in both the source aperture and the sky annulus. After performing the aperture photometry, magnitudes from saturated sources were replaced by the correct values from the unsaturated images.

The full data tables containing the integrated photometry and analysis results of all clusters are available, at http://www.astro.physik.uni-goettingen.de/ $\sim$ galev/panders/NGC1569/NGC1569_cluster_data.dat .

Note that, in order to determine model magnitudes from our model spectra (see Sect. 4.3.1), we adopt the full filter response functions, including the red leak in F336W. Therefore, we need not attempt to correct for the red leak of the F336W filter. In general, the impact of the red leak is negligible for spectra dominated by early-type stars such as a starburst spectrum (e.g. Eskridge et al. 2003, de Grijs et al. 2003a). In addition, any ambiguity originating from 
the red leak is resolved by our spectral energy distribution (SED) analysis tool, which takes into account the whole SED.

From the 47 clusters of H00, only 17 are matched in our source list. Roughly a dozen of the H00 clusters lie outside of our final FoVs from the combined HST data, and hence are not included in this work. Another $9 \mathrm{H} 00$ clusters are in the close vicinities of larger clusters, and are therefore rejected by our selection criteria; the sizes of most of these clusters, as estimated by $\mathrm{H} 00$, are close to our lower size limit of 1.7 pixel. In addition, the source selection criteria are not described in detail by H00, and hence comparison is difficult or impossible. By comparing the photometry of the clusters in common, after correctionfor the different distance moduli and extinction values considered, we find the H00 values to be brighter, in general, than ours, which can be attributed to different apertures used for the photometry (mostly larger in the case of H00), see Fig. 4.3. H00 attempted to extract the contribution of nebular emission from their science images, in particular of $\mathrm{H} \alpha$ emission, using appropriate narrow-band filters to estimate the approximate $\mathrm{H} \alpha$ line intensity and its continuum level. However, further details are not given in their paper. This makes comparison difficult, but since we account for nebular emission in our models the parameter analysis is not affected. The error bars in Fig. 4.3 are calculated from the photometric errors in $\mathrm{H} 00$ and from our work. In general, the median photometric errors for our clusters are of the order of 0.05 mag except for the F336W filter where they are around $0.1 \mathrm{mag}$. The photometric errors considered in this paper include Poissonian noise in the background and in the source flux itself, and variations in the background level.

Only in a few cases the correlation of brighter clusters with larger source annuli in the H00 sample does not hold. The SSCs are surrounded by smaller substructures and superimposed on generally high background levels, where reliable aperture photometry is difficult, in terms of both source and background fluxes. In such cases, the results are very sensitive to the exact positions and sizes of the source and background annuli.

We will now discuss the few true "outliers" in Fig. 4.3 in more detail. H00 cluster "no. 9" lies close to another bright source (probably a bright star), hence contamination of the source flux of the cluster by the other source is likely.

Cluster "no. 6" is located in a region with very strong gas emission (and is a strong emitter itself because of its young age; we determine an age of $4 \mathrm{Myr}$ ), according to the false-colour image including $\mathrm{H} \alpha$ emission of $\mathrm{H} 00$ (their Fig. 2). This gas emission might lead to misassignment of flux to either the source or the background flux.

These differences in the integrated cluster photometry between the H00 values and ours reflect the advantages of a visual inspection of each cluster in the complicated environments associated with ongoing starbursts, compared to automated cluster photometry. Non-cluster features, such as crowding or extended gas emission, are taken into account more reliably.

In Anders et al. (2003) we show that SEDs of young clusters exhibit a "hook" near the B band (see also Fig. 4.6). Tracing this hook is of the highest importance for the determination of accurate cluster parameters. The deep observations in F380W and the additional F336W observations are vital for tracing this hook. In addition, having near-infrared (NIR) data available further improves the analysis. For the majority of our clusters we have NIR data at hand. Hence, the observations available to us for NGC 1569 are ideally suited 


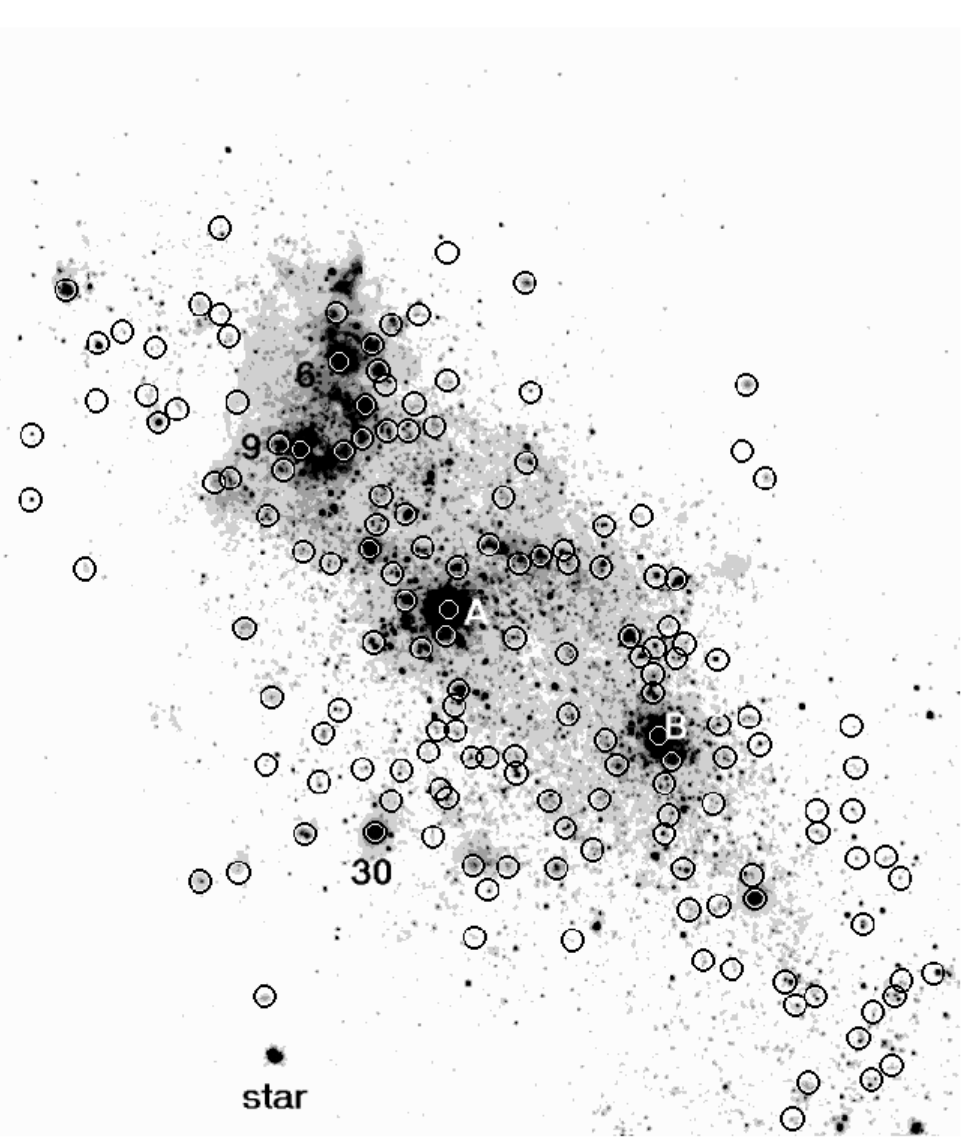

Figure 4.2: Image of the inner part of NGC 1569 (our small FoV) with the positions of the clusters marked. Some clusters (and one apparent star) are labelled. North is marked by the arrow, east is indicated by the line perpendicular to the arrow.

to produce accurate cluster parameters.

\subsubsection{Sample completeness}

The completeness of the exposures was determined by adding artificial sources of known brightness to the saturated science images, and subsequent source identification. This analysis was performed using the appropriate IRAF and IDL tasks in a similar way as for the source selection of the real clusters. By cross-correlating the list of retrieved objects with the input artificial sources, the fraction of recovered artificial sources was determined. The results for the small FoV are shown in Fig. 4.4. The completeness limits for the large FoV are similar.

These completeness curves were corrected for the effects of blending or superposition of multiple randomly placed artificial PSFs as well as for the superposition of artificial PSFs on top of genuine objects. For the analysis in this 


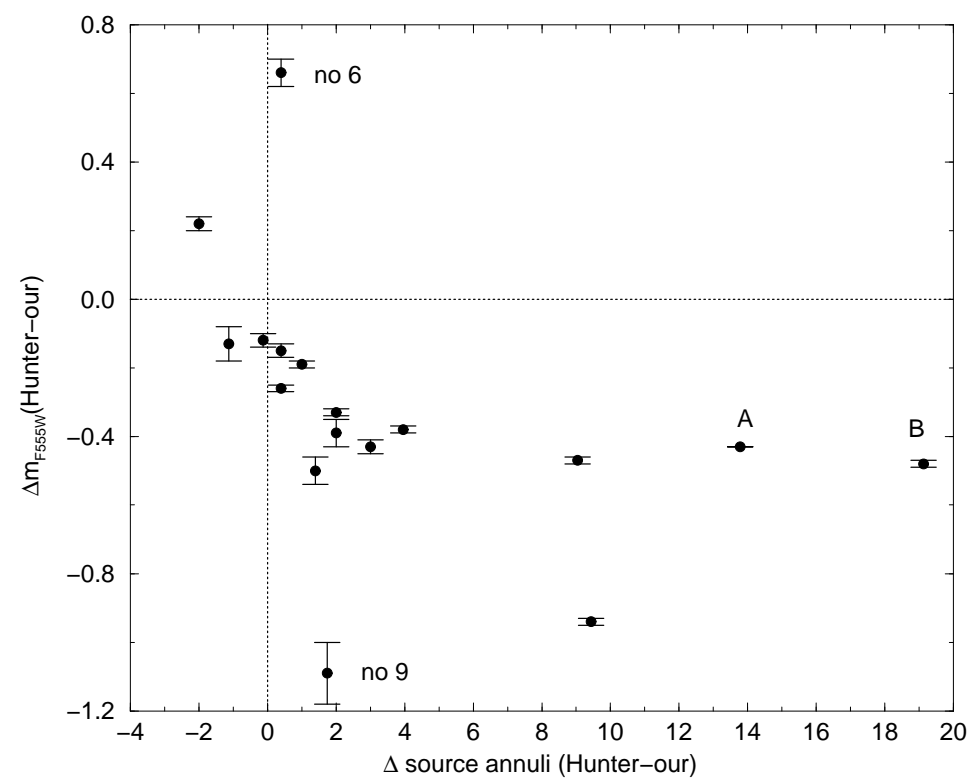

Figure 4.3: Comparison of the cluster photometry obtained from H00 and in this work. Displayed is the dependence of the magnitude difference on the difference of the source apertures used. Naming of 4 individual clusters is following H00. Lines are included to guide the eye.

paper, completeness limits at 90 per cent will be considered.

\subsection{Parameters of the young clusters}

\subsubsection{Cluster analysis algorithm}

We applied our maximum-likelihood algorithm to the magnitudes of the cluster candidates found in Section 4.2.2. This algorithm and several tests of it are described in Anders et al. (2003). Only a summary of the method is given here.

We use the evolutionary synthesis models of our GALEV code, described in detail in Schulz et al. (2002), with important additions regarding the treatment of gaseous emission in the early stages of cluster evolution presented in Anders \& Fritze - v. Alvensleben (2003). We also calculate model magnitudes with internal dust extinction, by adopting the starburst extinction law of Calzetti et al. (2000), assuming a foreground screen geometry. Our extinction estimates are therefore, strictly speaking, lower limits. Galactic extinction is taken into account by dereddening the observations using the appropriate Galactic extinction values from Schlegel et al. (1998).

Our models are based on stellar isochrones from the Padova group which include the thermally-pulsing AGB-phase shown to be vital to correctly predict the colours of clusters with ages between $200 \mathrm{Myr}$ and 1 Gyr (see Schulz et al. 2002). Throughout the paper we adopt a Salpeter initial mass function (IMF) with lower mass limit $M_{\text {low }}=0.15 M_{\odot}$ and upper mass limit $M_{\text {up }} \approx 50-70 M_{\odot}$, 


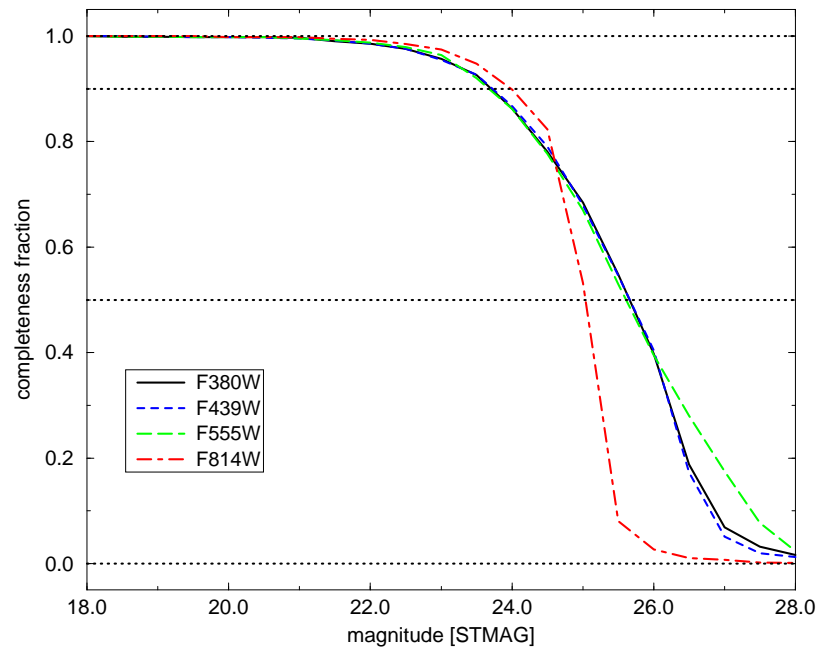

Figure 4.4: Completeness curves for NGC 1569. The different line styles refer to different passbands, as indicated in the legend. These completeness curves apply to the small FoV. The horizontal lines indicate 0, 50, 90 and 100 per cent completeness. Magnitudes are given in STMAG, the standard HST zero-point system, based on a flat reference spectrum.

determined by the upper mass limit of the Padova isochrones $\left(M_{\mathrm{up}}=50 M_{\odot}\right.$ for the highest metallicity $\mathrm{Z}=0.05, M_{\mathrm{up}} \approx 70 M_{\odot}$ for the lower metallicities). Adopting a different IMF, however, affects the derived (absolute) masses of the clusters, but the effect on the other parameters, and on the relative mass distribution, is negligible. The mass offset by assuming a different IMF can easily be derived analytically. The spectral library used is given in Lejeune et al. $(1997,1998)$. The emission line coefficients for low metallicities are taken from Izotov et al. (1994, 1997, 1998), and from Stasińska (1984) for metallicities $\geq 0.008$. The Lyman-continuum photon output was calculated by Schaerer \& de Koter (1997), and recently confirmed by Smith et al. (2002), see Anders \& Fritze - v. Alvensleben (2003) for details.

Our models assume a well-populated IMF, which is an over-simplified assumption for systems with small numbers of bright stars, as shown e.g. by Cerviño et al. (2002) and Cerviño \& Valls-Gabaud (2003). Small-number statistics and stochastic effects for bright stars, such as Wolf-Rayet stars or supergiants, introduce additional model magnitude dispersions, which scale inversely with the mass of the cluster. However, no complete study for all magnitudes and input parameters has yet been performed.

We construct SEDs from these models with an age resolution of 4 Myr for ages from 4 Myr up to 2.36 Gyr, and with 20 Myr resolution for older ages (up to a maximum age of $14 \mathrm{Gyr})$. The extinction resolution is $\Delta \mathrm{E}(\mathrm{B}-\mathrm{V})=0.05$ $\mathrm{mag}$, for $\mathrm{E}(\mathrm{B}-\mathrm{V})$ in the range of $0.0-1.0 \mathrm{mag}$. The adopted metallicities are $[\mathrm{Fe} / \mathrm{H}]=-1.7,-0.7,-0.4,0.0,0.4$, as given by the Padova isochrones used (for 
a general description of the stellar models see Bertelli et al. 1994 and Girardi et al. 2000; for details about the isochrones in our models see Schulz et al. 2002).

When comparing our model SEDs with the observed SEDs we first determine the mass of the cluster by shifting the model SED onto the observed SED. This shift is equivalent to scaling the model's mass to the cluster mass.

Each of the models is now assigned a certain probability to be the most appropriate one, determined by a likelihood estimator of the form $p \sim \exp \left(-\chi^{2}\right)$, where $\chi^{2}=\sum \frac{\left(m_{\text {obs }}-m_{\text {model }}\right)^{2}}{\sigma_{\text {obs }}^{2}}$. Clusters with unusually large "best" $\chi^{2}$ are rejected, since this is an indication of calibration errors, features not included in the models (such as Wolf-Rayet star dominated spectra, objects younger than $4 \mathrm{Myr}$ etc.) or problems due to the limited parameter resolutions. The lower cut-off is set to a total probability $=10^{-20}$, corresponding to a $\chi_{\text {best }}^{2} \geq 46$. The total probability per cluster is then normalised.

Subsequently, the model with the highest probability is chosen as the "bestfitting model". Models with decreasing probabilities are summed up until reaching 68.26 per cent total probability ( $=1 \sigma$ confidence interval) to estimate the uncertainties of the best-fitting model. These uncertainties are in fact upper limits, since their determination does not take into account effects like the existence of several "solution islands" for one cluster (such as e.g. the age-metallicity degeneracy, see Section 4.3.2), and discretisation in parameter space.

Several passband combinations (containing at least 4 passbands) were used for the analysis, to minimise the impact of statistical effects on the errors and calibration errors. A minimum of 4 passbands is required to determine the 4 free parameters age, metallicity, extinction and mass independently (see also Anders et al. 2003, de Grijs et al. 2003a). We caution that these passband combinations must not be biased to contain mainly short-wavelength filters or mainly longwavelength filters. Coverage of the entire optical wavelength range, if possible with the addition of ultra-violet (UV) and NIR data, is most preferable (de Grijs et al. 2003a). We select the passband combinations starting with all available filters, and then rejecting passbands starting with the shallowest exposures and exposures not covering the entire combined FoV.

Only clusters with observational errors $\leq 0.2 \mathrm{mag}$ in all passbands of a particular combination were included to minimise the uncertainties in the results. For each combination, the best-fitting models and the associated parameter uncertainties were determined. For a certain cluster all best-fitting models (and the associated uncertainties), originating from the different passband combinations, were compared. For each of these best-fitting models the product of the relative uncertainties $\left(\frac{\text { age }^{+}}{\text {age }^{-}} \times \frac{\text { mass }^{+}}{\text {mass }^{-}} \times \frac{\mathrm{Z}^{+}}{\mathrm{Z}^{-}}\right)$was calculated (the superscripts indicate the upper limits $\left(^{+}\right)$and the lower limits $\left(^{-}\right)$, respectively) The relative uncertainty of the extinction was not taken into account, since the lower extinction limit is often zero. For each cluster, the data set with the lowest value of this product was adopted as the most representative set of parameters (and parameter uncertainties). In cases where the analysis converged to a single model, a generic uncertainty of 30 per cent was assumed for all parameters in linear space, corresponding to an uncertainty of ${ }_{-0.15}^{+0.1}$ dex in logarithmic parameter space. See also de Grijs et al. (2003a,b) for an application of this algorithm to NGC 3310 and NGC 6745, and Anders et al. (2003) for a theoretical analysis of its reliability.

The figures presented in this paper are based on the overall best values. 


\subsubsection{Parameter distributions}

In total, we identified and analysed 144 clusters in the small FoV, and 157 clusters in the large FoV. All of these clusters meet the minimum error criterion in at least one passband combination, while 24/22 additional clusters (small FoV/large FoV) were rejected by this criterion. Every accepted cluster was matched with representative single stellar population models by our algorithm.

We mainly used 3 passband combinations for the analysis: all 7 passbands ("7mag"), the combination of F380W, F439W, F555W, F814W, and F160W ("UBVIH") and the most restricted combination of F380W, F439W, F555W, and F814W ("UBVI"). In the small FoV we managed to match 62 clusters using 7mag; 24 additional clusters were matched using UBVIH (since either their F336W or their F110W magnitudes had errors larger than $0.2 \mathrm{mag}$ ). The remaining 58 clusters in the final sample for the small FoV could only be analysed without NIR information. These latter clusters are mostly concentrated towards the corners of this FoV, where due to the rotation applied to the NICMOS images there is no NIR information available. To summarise, if NIR information is available, passband combinations including the NIR filter NICMOS F160W give the best results in almost all cases, based on their relative uncertainty product.

For the small FoV we checked the uncertainties inherent to the analysis routine. We find median uncertainties of 1 step in metallicity (as a reminder, the metallicities used are $[\mathrm{Fe} / \mathrm{H}]=-1.7,-0.7,-0.4,0.0,0.4), 0.1$ mag in extinction $\mathrm{E}(\mathrm{B}-\mathrm{V})$, a factor of 3 in age (corresponding to a logarithmic uncertainty of 0.5 ), and a mass uncertainty of a factor of 2.3 (corresponding to a logarithmic uncertainty of 0.35 ). This is in good agreement with theoretical tests of our algorithm (see Anders et al. 2003 for more details). We have chosen the bin sizes in the following figures based on these uncertainty estimates.

\section{Parameter distributions of the entire sample in the small FoV}

In Fig. 4.5 the derived parameter distributions of the clusters in the small FoV are shown (open histograms).

The metallicity distribution (Fig. 4.5, $[\mathrm{Fe} / \mathrm{H}]$ ) is dominated by significantly subsolar-abundance clusters. The high-metallicity part is dominated by the youngest clusters (ages $\leq 8 \mathrm{Myr}$ ), as can be seen from a comparison with the shaded histograms. We attribute this to the age-metallicity degeneracy. To further quantify the associated effects we analysed the fractions of clusters without NIR data (and hence less reliable parameters, especially the metallicity is fixed most effectively using NIR data) in certain age and metallicity bins. We found the youngest ages (at $4 \mathrm{Myr} \approx 63$ per cent, at $8 \mathrm{Myr} \approx 50$ per cent) and the highest metallicities (for $\mathrm{Z}=0.02=\mathrm{Z}_{\odot} \approx 56$ per cent, for $\mathrm{Z}=0.05 \approx 68$ per cent) to be dominated by clusters for which no NIR photometry with uncertainties $\leq 0.2$ mag was available. In the other bins, the average fraction of clusters without NIR data is around 30 per cent.

Almost all clusters were produced in the last $25 \mathrm{Myr}$ in a very intense burst. Only 21 clusters $(\approx 15$ per cent) are older. This is consistent with the existing evidence regarding the ages of the SSCs, and the dynamical age of certain morphological features like arcs and superbubbles (de Vaucouleurs et al. 1974, Waller 1991, Tomita et al. 1994, Heckman et al. 1995). While these morphological features are thought to be evidence for self-propagating star-formation, our age determination does not provide further proof of this scenario, since no spatial concentration of clusters at any given age is observed. 

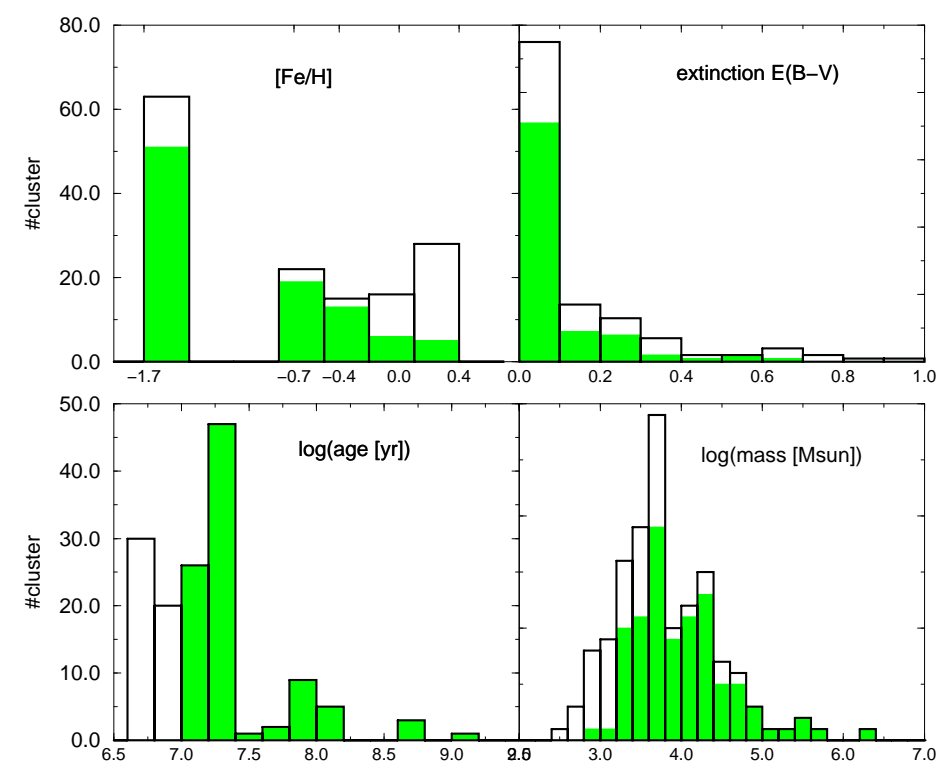

Figure 4.5: Parameter distributions of the clusters in the small FoV; open histograms: all ages, shaded histograms: only ages $>8$ Myr.

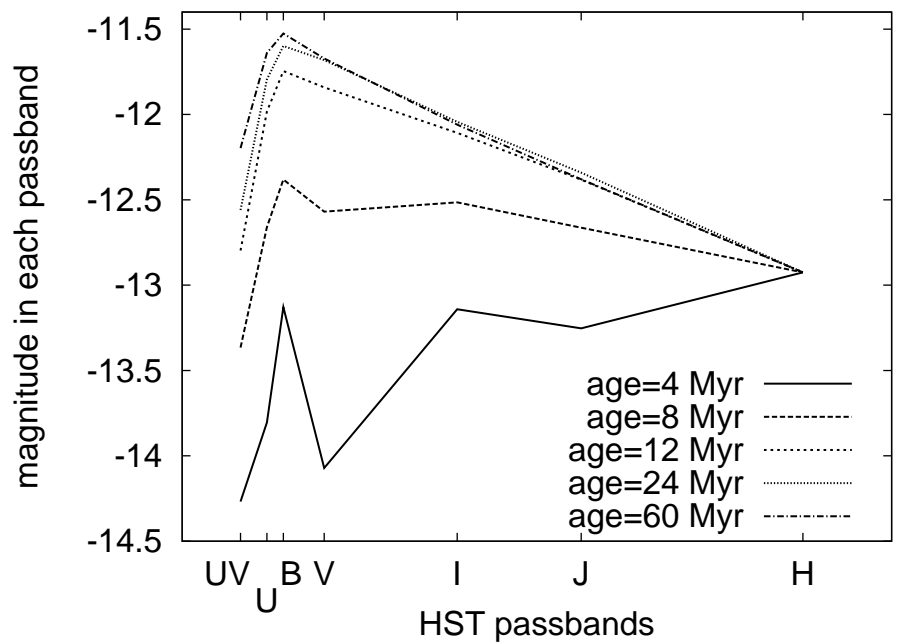

Figure 4.6: Model SEDs for $\mathrm{Z}=0.004$ and 5 different young ages. SEDs are shifted to coincide at the $H$ band.

In Figure 4.6 we present a number of model SEDs for the low-metallicity environment of NGC 1569 and the young ages typical for its star clusters. With typical observational errors of 0.05-0.1 mag these SEDs are clearly distinguishable. The UV range is crucial for this distinction, in agreement with our theoretical analysis in Anders et al. (2003). The deep observations of NGC 1569 
in the F380W filter (approximately equivalent to the $U$ band) are vital for the accuracy of our results.

Colour-magnitude diagram analyses yield more extended starburst histories for NGC 1569 starting up to $150 \mathrm{Myr}$ ago, and ending around 5-10 Myr ago, with ongoing low-level star formation for the last 1.5 Gyr (Vallenari et al. 1996, Greggio et al. 1998, Aloisi et al. 2001). Since such extended burst scenarios are not supported by our determinations of the cluster ages, we rather associate this with the low-level star cluster formation for clusters with $\log$ (age) $\geq 7.4$ and the secondary peak of star cluster formation at around $100 \mathrm{Myr}$ ago.

The extinction towards the NGC 1569 clusters is low: 73 per cent of the clusters have $\mathrm{E}(\mathrm{B}-\mathrm{V}) \leq 0.1$. Only 10 clusters $(\approx 7$ per cent) have $\mathrm{E}(\mathrm{B}-\mathrm{V}) \geq$ 0.5 , and all of these are young (ages $\leq 16 \mathrm{Myr}$, with most of them as young as $4 \mathrm{Myr})$.

The masses of the cluster candidates are low compared to Galactic globular clusters, which have a Gaussian shaped mass distribution with

$$
\left\langle\log \left(M_{\mathrm{GC}}\left[\mathrm{M}_{\odot}\right]\right)\right\rangle_{\mathrm{MW}} \simeq 5.5
$$

and

$$
\sigma\left(\log \left(M_{\mathrm{GC}}\left[\mathrm{M}_{\odot}\right]\right)_{\mathrm{MW}}\right) \simeq 0.5
$$

(Ashman et al. 1995). In the case of the star cluster sample in NGC 1569 we are likely observing a system of open cluster-type objects rather than globular cluster progenitors. Only 4 objects have masses in excess of $\log \left(\operatorname{mass}\left[M_{\odot}\right]\right)=5.47$.

An even more remarkable result is shown in Fig. 4.7. There is significant evidence that the clusters formed at the onset of the burst are, on average, more massive than the clusters formed more recently. The vertical solid lines in the top two panels indicate the completeness limits for ages of $4 \mathrm{Myr}$ and $24 \mathrm{Myr}$, respectively. The dashed lines indicate completeness if one assumes an additional drop in completeness of a yet another $1.0 \mathrm{mag}$ due to the visual examination, because of a possible bias to reject preferentially fainter clusters. We would require an additional drop of 1 mag to explain the decrease at log(mass) $<3.2$ in the second age bin's shaded histogram. Alternatively, this drop might be caused by disruption of the lowest-mass clusters on time-scales as short as $25 \mathrm{Myr}$.

To investigate the significance of this change in mass function we display, in Fig. 4.8, a subset of clusters with age estimates that are entirely within the respective age bins. The right-hand panels show the most extreme configuration allowed by the $1 \sigma$ uncertainties of our mass estimates, where we assume that all clusters in the younger age bin have masses at the upper limit of the $1 \sigma$ mass uncertainty, and all clusters in the older age bin have masses at the lower limit of the $1 \sigma$ mass uncertainty range. An excess of clusters with $\log$ (mass) $\geq 3.8$ is still clearly visible. Quantitatively, this excess is significant at roughly the $10 \sigma$ level and cannot be explained even by worst-case Poisson-noise scenarios.

While the behaviour at the low-mass end remains debatable due to incompleteness effects, the excess of clusters with $\log ($ mass $) \geq 3.8$ in the intermediate age bin is significant. We will discuss a number of possible explanations for this excess in Section 4.5.

The "well-known" clusters 


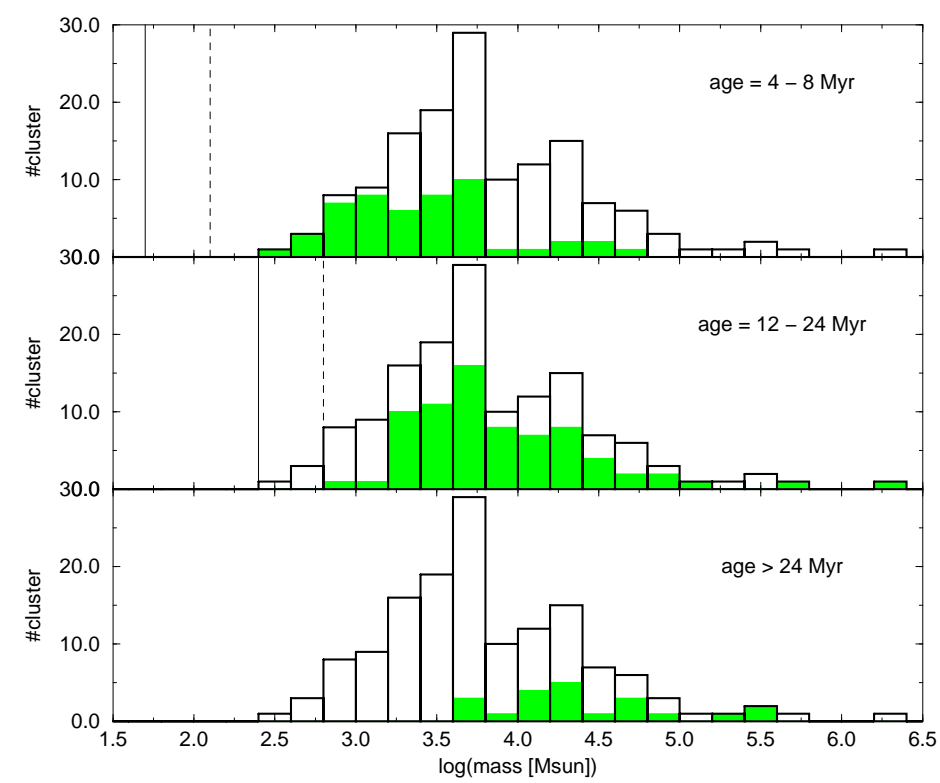

Figure 4.7: Mass functions of NGC 1569 cluster candidates in three age bins (shaded histograms; ages as indicated in each panel) and the total mass function (open histograms). Vertical lines indicate completeness limits (see text for details).

Since most information in the literature is available for the clusters "A", "B" and "no. 30", we compare our determinations for these clusters to those found in the literature in Table 2. The masses are taken from Ho \& Filippenko (1996) ("HF96") and Gilbert \& Graham (2001) ("GG01"). The ages are from H00 and Origlia et al. (2001).

The ages of the two SSCs determined in this work agree very well with those from the literature. The agreement is not as good for cluster "no. 30" (our determination indicates a significantly older age than the literature value), but since no uncertainties are given in the literature, a direct comparison is difficult. However, since cluster "no. 30" is located at some distance from the bar of the galaxy, where the vast majority of star clusters is concentrated, a formation earlier than the major burst is not unlikely.

We determine higher masses than inferred from kinematic studies. Three reasons are possible:

1. We systematically determine ages that are too old, and due to the rapid changes in the mass-to-light $(\mathrm{M} / \mathrm{L})$ ratio at these early stages the resulting masses are too high. This scenario is not supported by our age uncertainties.

2. We adopted an incorrect stellar IMF. If the low-mass slope of the IMF is shallower than Salpeter (see e.g. Kroupa et al. 1993), our mass estimates need to be reduced by a factor of roughly 2 (see de Grijs et al. 2003b). 


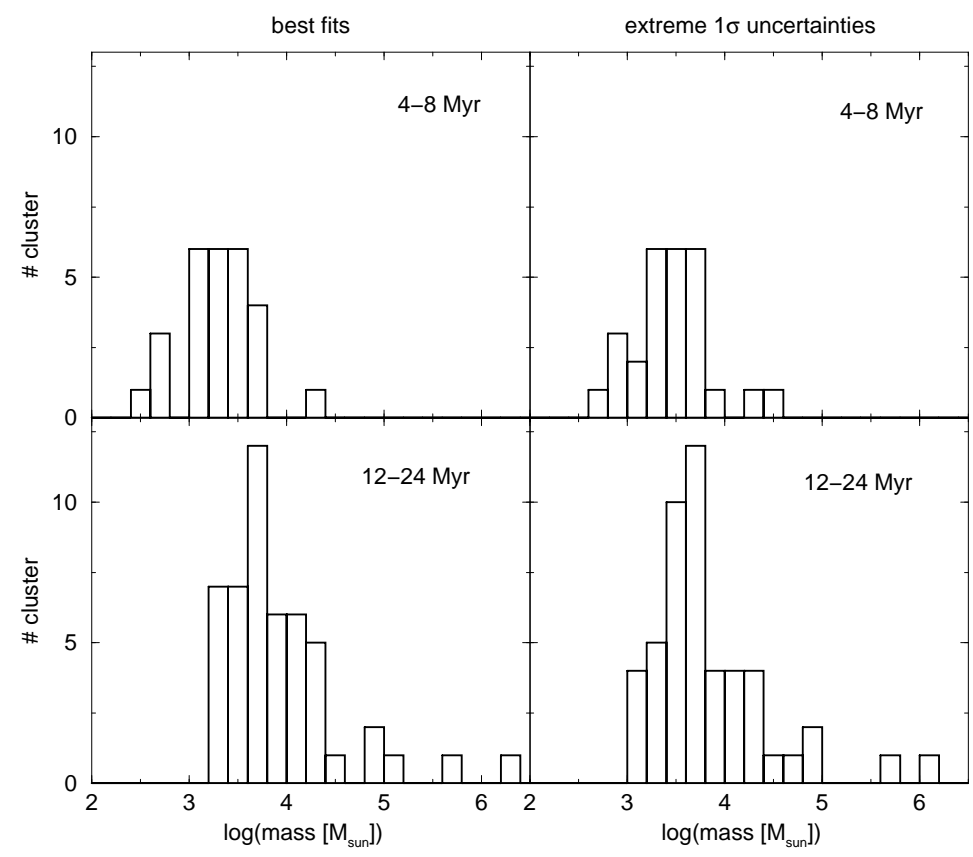

Figure 4.8: Mass functions of NGC 1569 cluster candidates in two age bins (as indicated), with age uncertainties entirely in this age bin. Left panels: best values. Right panels: Extreme cases allowed by uncertainty estimates (young ages: upper limits; older ages: lower limits). See text for details.

3. The masses calculated from the measured velocity dispersions are underestimated.

The kinematic masses of GG01 are about a factor of 2 smaller than ours, which is most likely within the uncertainties inherent to both methods (e.g. including the uncertainty in the IMF, evolutionary synthesis uncertainties, parameter uncertainties originating from the observation-model comparison, and uncertainties in the kinematic masses). A mass of $3.3 \times 10^{5} \mathrm{M}_{\odot}$ was derived by HF96 for SSC A. However, they did not take into account the substructure of this cluster, nor its impact on the velocity dispersion. They also assumed a different distance modulus, and a fixed sigma to correct for the impact of red supergiants, rather than measuring it from the autocorrelation function, as done by GG01 (although HF96 claim to give a lower mass limit only). Nevertheless, both studies assume complete virialisation of the clusters, which is likely not the case for clusters as massive and as young as these two SSCs (hence the dynamical mass estimate is likely an underestimate). A cluster's half-mass relaxation time is given by $T[\mathrm{yr}]=8 \times 10^{5} \frac{n^{1 / 2} \cdot R^{3 / 2}}{\langle m\rangle^{1 / 2} \cdot(\ln (n)-1)}$, with $n$ the number of stars in the cluster, $R$ its radius in pc and $\langle m\rangle$ the median mass of a star in the cluster. The radii are taken from de Marchi et al. (1997): 1.6 pc for SSC A1, $1.8 \mathrm{pc}$ for $\mathrm{SSC} \mathrm{A} 2 / \mathrm{B}$. We assume that the half-light radius approximates the half-mass radius for young clusters (cf. de Grijs et al. 2002a), a median mass of a star in the cluster is $\approx 0.3 \mathrm{M}_{\odot}$ (for a well-populated IMF ranging from 0.15 
Table 4.2: Comparison of parameters of clusters with literature values

\begin{tabular}{|c|c|c|c|c|}
\hline cluster & $\begin{array}{c}\text { Average } \\
\text { Literature }\end{array}$ & $\begin{array}{c}\text { Uncertainties } \\
\text { Literature }\end{array}$ & $\begin{array}{c}\text { Best values } \\
\text { This work }\end{array}$ & $\begin{array}{c}\text { Uncertainties } \\
\text { This work }\end{array}$ \\
\hline \multicolumn{5}{|l|}{ age $[\mathrm{Myr}]$} \\
\hline $\mathrm{SSC} \mathrm{A}^{a}$ & 7 & $4-20$ & 12 & $8-16$ \\
\hline SSC B & 15 & $10-30$ & 12 & $12-28$ \\
\hline No. 30 & 30 & - & 92 & $28-112$ \\
\hline cluster & "HF96 & "GG01 & $\begin{array}{l}\text { Best values } \\
\text { This work }\end{array}$ & $\begin{array}{l}\text { Uncertainties } \\
\text { This work }\end{array}$ \\
\hline \multicolumn{5}{|l|}{$\operatorname{mass}\left[M_{\odot}\right]$} \\
\hline $\mathrm{SSC} \mathrm{A}^{a}$ & $3.3 \times 10^{5}$ & $8.3 \times 10^{5 b}$ & $1.6 \times 10^{6}$ & $(1.1-2.1) \times 10^{6}$ \\
\hline SSC B & - & $2.3 \times 10^{5}$ & $5.6 \times 10^{5}$ & $(5.6-8.8) \times 10^{5}$ \\
\hline No. 30 & - & - & $3.6 \times 10^{5}$ & $(2.8-6) \times 10^{5}$ \\
\hline
\end{tabular}

Notes: ${ }^{a}$ Analysis in this work converged to 1 model only; 30 per cent uncertainties are assumed.

${ }^{b}$ Sum of both subcomponents.

$\mathrm{M}_{\odot}$ to $70 \mathrm{M}_{\odot}$ [upper mass limit given by Padova isochrones for low metallicity]), masses are adopted from GG01 $\left(3.9 \times 10^{5} \mathrm{M}_{\odot}\right.$ for $\mathrm{A} 1,4.4 \times 10^{5} \mathrm{M}_{\odot}$ for A2 and $2.3 \times 10^{5} \mathrm{M}_{\odot}$ for $\mathrm{B}$ ), numbers of stars in the clusters are calculated from the total cluster mass and the characteristic stellar mass. These values result in half-mass relaxation times of 250-400 Myr, well in excess of the expected ages of these clusters by more than a factor of 20. However, this relaxation model does not account for effects of mass segregation (for observational evidence of significant $a b$ initio mass segregation in a sample of young LMC clusters see de Grijs et al. 2002b), or radial dependences of the relaxation time-scales. Hence uncertainties are large. Our data do not allow to discriminate between these sources of uncertainties.

The best-fitting metallicity for NGC 1569 derived from CMD analyses is $[\mathrm{Fe} / \mathrm{H}]=-0.7$ (Greggio 1998, Aloisi 2001, both using Padova tracks). This agrees well with spectroscopic abundance measurements by Kobulnicky \& Skillman (1997) and Devost et al. (1997). Both teams measure abundances of around $12+[\mathrm{O} / \mathrm{H}]=8.2$, corresponding to $[\mathrm{Fe} / \mathrm{H}]=-0.7$. We find both SSC B and no. 30 best matched by models with $[\mathrm{Fe} / \mathrm{H}]=-0.4$, and hence comparable to the literature values cited within the uncertainties associated with the methods. SSC A is best matched by a model with the lowest metallicity available $([\mathrm{Fe} / \mathrm{H}]=-1.7)$. This might reflect the uncertain character of this star cluster, which may consist of two subclusters (de Marchi 1997), but certainly contains two very distinct populations (González Delgado et al. 1997, de Marchi et al. 1997, H00, Maoz 2001): Wolf-Rayet stars and red supergiants. Hence a simple single stellar population model is probably not appropriate for this cluster. In addition, Wolf-Rayet stars are not specifically marked in the Padova isochrones, and the treatment of red supergiants by the Padova group differs from that of e.g. the Geneva group. The treatment of these stars is not yet beyond debate.

Comparison with other subsamples and the age-metallicity degeneracy

Figure 4.9 compares the parameter distributions of clusters in the small FoV with the ones from the large FoV. The major difference between these two FoVs is not just the spatial coverage, but - more importantly - for all clusters in the large FoV the NIR information is either unavailable or omitted.

While the extinction and $\log$ (mass) distributions are fairly similar, the metal- 

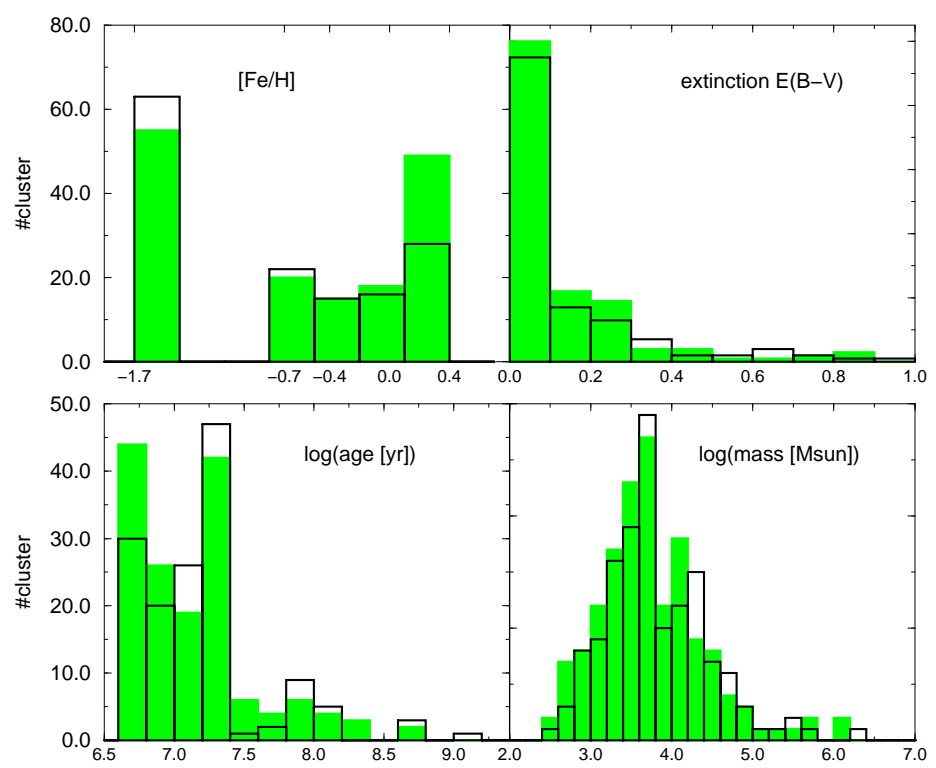

Figure 4.9: Parameter distributions of the two FoVs: open histograms = small FoV (including NICMOS coverage), shaded histograms = large FoV (without NICMOS coverage).

licity and age distributions show a significant shift. The clusters in the large FoV seem to have, on average, higher metallicity and younger ages. While these parameters are expected to correlate, a closer inspection of the data reveals a different situation. Comparing the same clusters in both the small and the large FoVs shows that of the clusters classified as having super-solar metallicity in the large FoV only 48 per cent are classified as solar/super-solar in the small FoV. Instead, some 33 per cent of them were originally classified as having the lowest possible metallicity $[\mathrm{Fe} / \mathrm{H}]=-1.7$. In addition, of the 28 clusters in the small FoV's super-solar metallicity bin, 19 (68 per cent) have no NIR information available (due to the rotated NICMOS FoV). Single stellar population models run close (and partly intersect each other) in colour evolution for different metallicities at early phases. As widespread super-solar abundances seem fairly implausible in this kind of dwarf galaxy, we strongly suspect these results to be a clear sign of the age-metallicity degeneracy. This degeneracy can only be broken by using NIR observations, in addition to UV-optical data, in particular to constrain the metallicity of the clusters properly. From this comparison we strongly recommend the use of NIR facilities for multi-band photometry to determine reliable cluster parameters from broad-band photometry, at least for clusters as young as in our sample.

\section{Investigating certain metallicity range restrictions}

To evaluate the robustness of our determinations, we performed cluster analysis with restricted metallicity ranges. The results for the log(age) and $\log$ (mass) distributions are shown in Fig. 4.10. While the rejection of the super-solar/solar metallicity range is justified physically (since large numbers of high-abundance 
clusters are not expected in a dwarf galaxy environment, such as in NGC 1569), the rejection of the lowest metallicity is a more theoretical exercise (since subsolar metallicity ranges cannot be omitted a priori).

These tests confirm the general properties obtained without restrictions in metallicity space, proving the robustness of our analysis method. The extinction (not shown) and $\log$ (mass) distributions are very similar, with only minor changes. The $\log$ (age) distributions confirm the onset of the major burst 24 Myr ago, and a minor burst around 100 Myr ago. The detailed structure of the major burst, however, depends on the metallicities allowed. Rejection of high metallicities leads to a depopulation of the youngest age bins. This is related to the study of the impact of the availability of NIR data on the derived parameters (Section 4.3.2). Rejection of the lowest metallicity leads to a distribution skewed to younger ages, to balance the mean higher metallicity.

The importance of robust, independent metallicity determinations can also be seen from Fig. 4.11, which shows the parameter distributions for the clusters in the small FoV, assuming solar metallicity (shaded histograms). The open histograms are the distributions from the same sample without such restrictions, for comparison. Major changes are visible in the log(age) (a clear shift towards younger ages for the metallicity-restricted values) and $\log$ (mass) distributions (towards lower masses, which is a consequence of the younger ages, in conjunction with the associated rapidly changing $\mathrm{M} / \mathrm{L}$ ratios).

To summarise, we have analysed a large sample of star clusters in NGC 1569 by comparing the observed cluster SEDs with an extensive grid of model SEDs to determine the cluster ages, metallicities, internal extinction values and masses in a robust and homogeneous way. We have presented the best-fitting values for the clusters, and compared them to the results with restricted parameter spaces or wavelength coverage. We conclude that we can determine ages (and hence the star cluster formation history; bursty, with a major peak starting 25 Myr ago, and a minor peak around 100 Myr ago), masses (similar to open cluster-type objects), extinction values (the average internal extinction is found to be low) and metallicities (significantly sub-solar; however, the impact of the age-metallicity degeneracy is clearly seen, especially for clusters without NIR data) robustly, with well-understood uncertainties.

\subsection{Cluster disruption vs. fading}

We applied the method of Boutloukos \& Lamers (2003) regarding cluster detectability limited by fading due to stellar evolution and cluster disruption to our cluster sample from the small FoV. The results are shown in Figs. 4.12 and 4.13. Since the total number of clusters is small, statistics are relatively poor, but consistent.

Figure 4.12 shows the number of clusters formed per year, as a function of $\log$ (age). The fading lines are based on the slope given by Boutloukos \& Lamers (2003) for the V-band (slope $\alpha_{\text {fade }}=-0.648$ ) and are shifted vertically to best match the data. The disruption line is a fit to the data points with $\log ($ age $) \geq$ 7.2 (with slope $\alpha_{\text {disr }}=-2 \pm 0.2$ ).

Since the Boutloukos \& Lamers (2003) method is based on the assumption of a constant cluster formation rate, which is not valid in our case, the interpretation of this figure is ambiguous: 

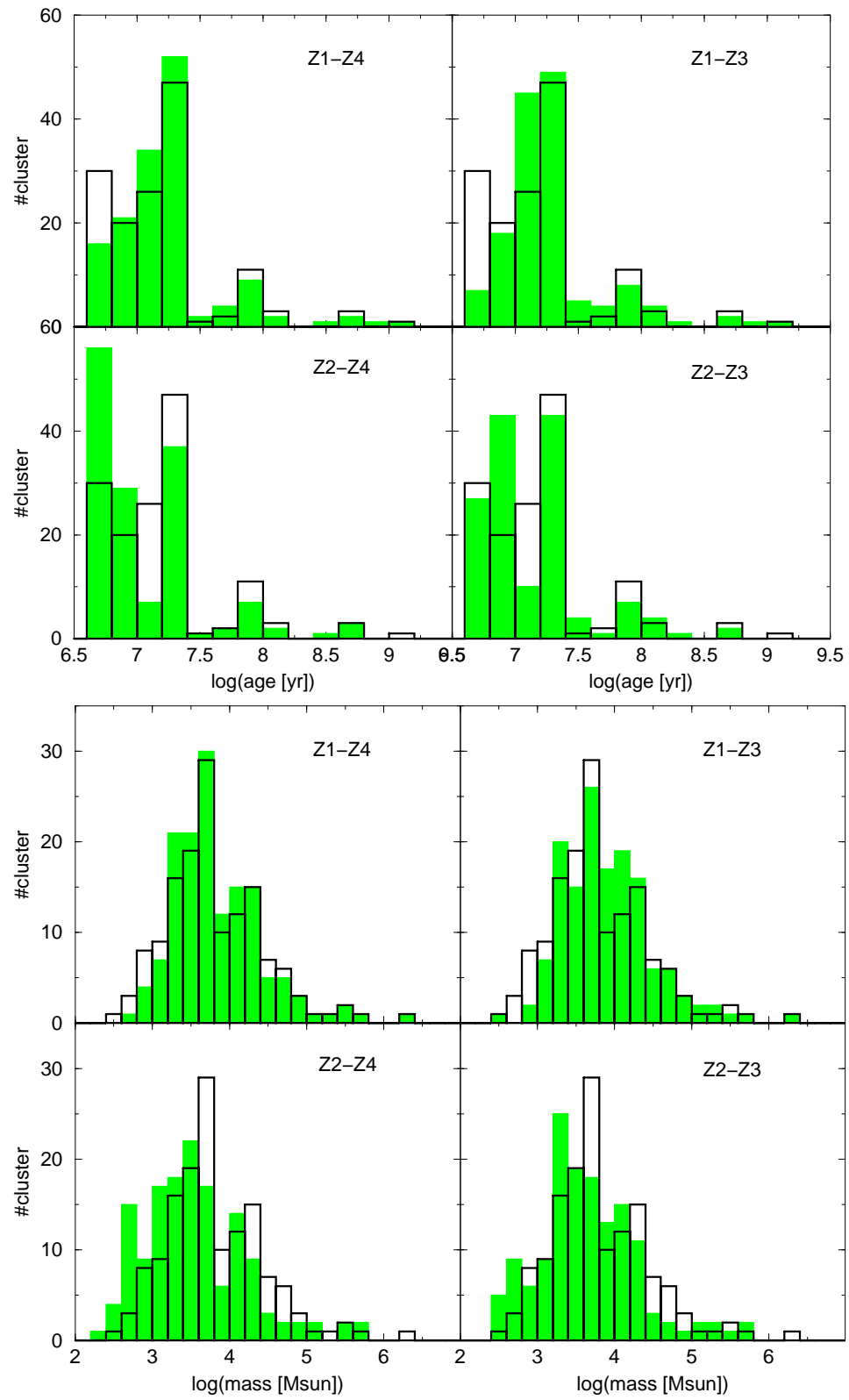

Figure 4.10: Comparison of parameters for certain metallicity restrictions (shaded histograms) for the small FoV. Upper panel: log(age) distributions, lower panel: $\log$ (mass) distributions. The allowed metallicity ranges are shown in the panels $(\mathrm{Z} 1 \equiv[\mathrm{Fe} / \mathrm{H}]=-1.7, \mathrm{Z} 2 \equiv[\mathrm{Fe} / \mathrm{H}]=-0.7, \mathrm{Z} 3 \equiv[\mathrm{Fe} / \mathrm{H}]=-0.4, \mathrm{Z} 4$ $\equiv[\mathrm{Fe} / \mathrm{H}]=0, \mathrm{Z} 5 \equiv[\mathrm{Fe} / \mathrm{H}]=+0.4)$. The results for no metallicity restriction are shown as open histograms, for comparison.

1. As a fading part (short-dashed line, for $\log ($ age $) \leq 7.2)$ and a disruption part (solid line), without any burst (which is unrealistic, compared to Fig. 

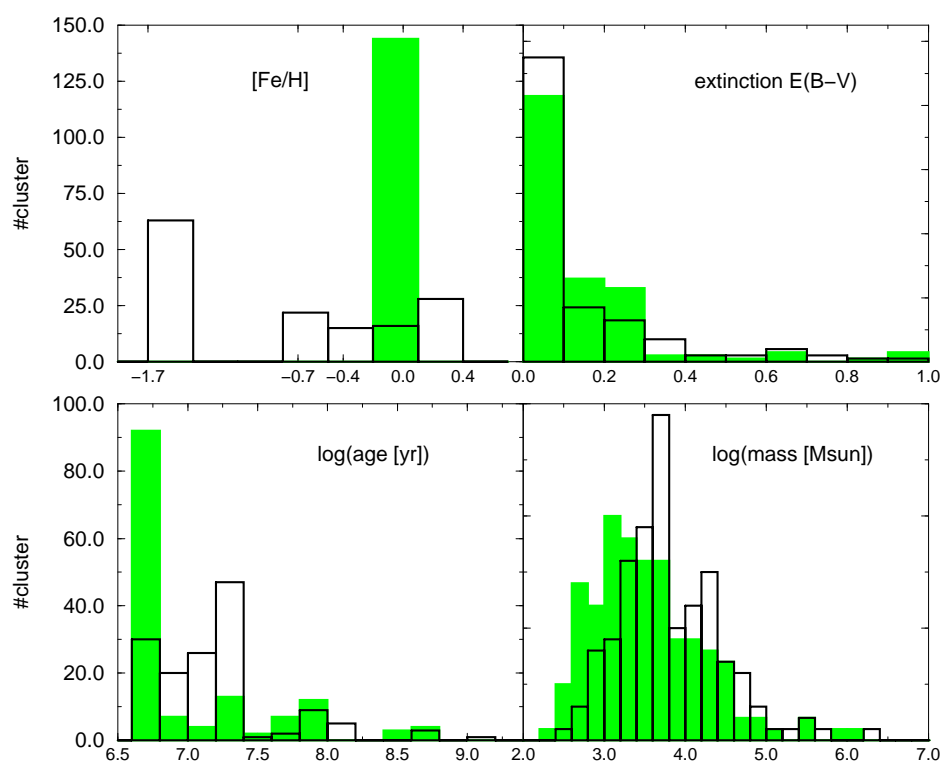

Figure 4.11: Comparison of parameters, derived with all metallicities allowed (open histograms) and with metallicity fixed to solar (shaded histograms)

$4.5)$.

2. With enhanced cluster formation in the recent past, which is only affected by fading (upper dot-dashed line) and offset from the low-level cluster formation fading line (middle dot-dashed line, for $\log ($ age) $\geq 7.5$ ), with subsequent cluster disruption (solid line).

3. With fading only, but with 2 bursts (upper dot-dashed line [log(age) $\leq$ $7.2]$ and middle dot-dashed line $[\log ($ age $) \simeq 7.8])$ and low-level cluster formation (lower dot-dashed line).

We treat the burst as a temporarily constant cluster formation rate (Boutloukos \& Lamers 2003; de Grijs, Bastian \& Lamers 2003), shifting the theoretical fading lines to fit our data in the respective age intervals.

Figure 4.13 shows the number of clusters formed per $M_{\odot}$ for 3 age bins. Due to small-number statistics the slope determinations are fairly uncertain, but comparable. The average slope, however, is shallower than expected (we find $\alpha_{M} \simeq-1.6$, other studies find slopes around -2 , see de Grijs et al. 2003b for a comparative compilation), but this might again be caused by the smallnumber statistics. Since the slopes for all age bins are comparable, we conclude that cluster disruption cannot yet have played a significant role, not even for the oldest clusters, which seems plausible in a low-density environment such as in NGC 1569. The lowest masses in each bin are clearly affected by incompleteness effects. The errors included in Fig. 4.13 are derived from Poissonian statistics in the respective mass bins. 


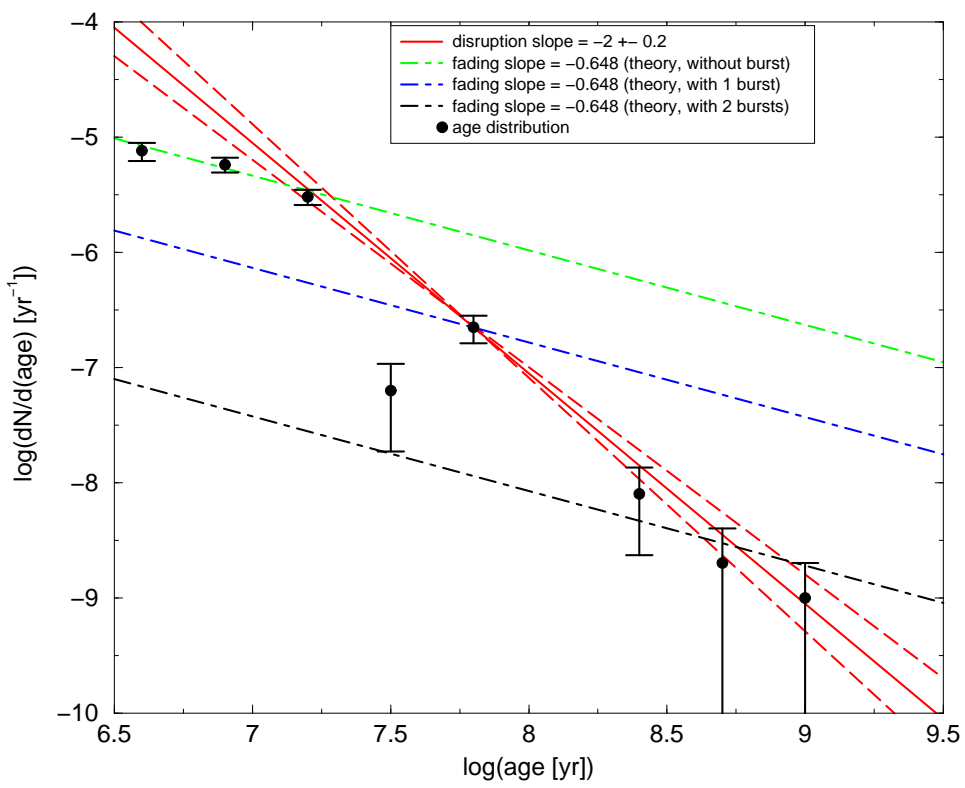

Figure 4.12: Cluster age distribution, affected by fading and cluster disruption. Linear relations are indicated, and slopes given in the legend; see text for details.

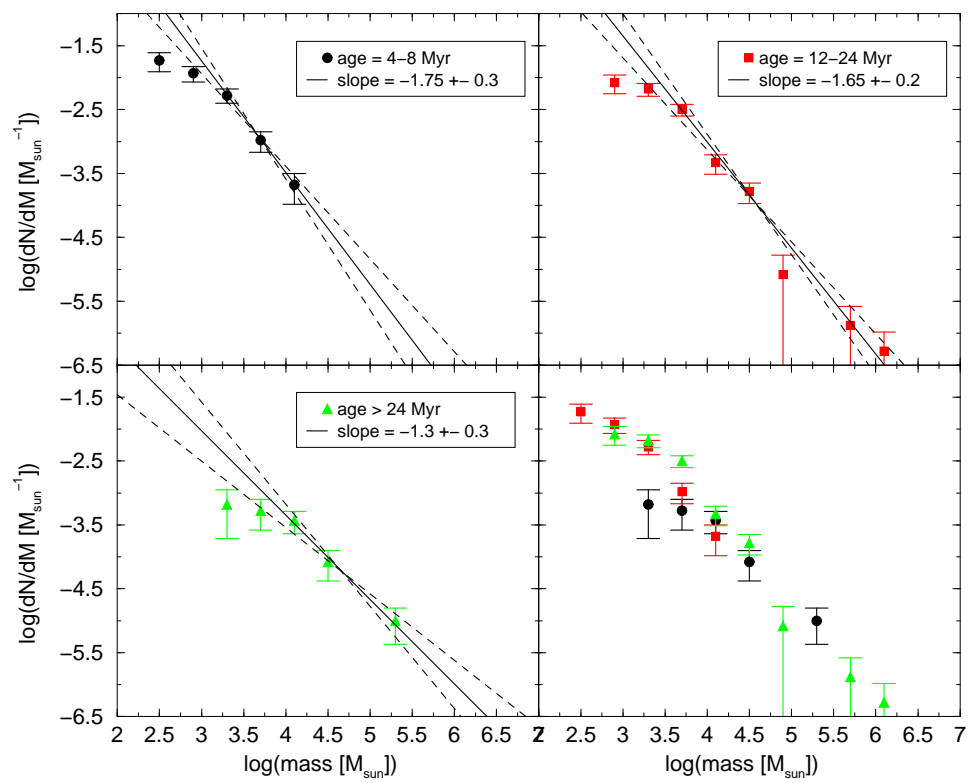

Figure 4.13: Cluster mass distribution for 3 different age bins, as indicated in the legends. Best-fitting slopes (and uncertainties) shown, as described in Section 4.4 . 


\subsection{The physical requirements for star cluster formation}

In Section 4.3.2 we observed a change in the mass function towards lower masses as the burst of star cluster formation proceeded. We performed a number of tests, to check whether this result (as shown in Fig. 4.7) is physically true or whether it might be only due to small-number statistics. First we counted the clusters in the mass bins $\log$ (mass $)=3.2-3.8$ and $\log$ (mass $)>3.8$, subdivided into the age bins age $=4-8 \mathrm{Myr}$ and age $=12-24 \mathrm{Myr}$ (as a reminder, we only have an age resolution of $4 \mathrm{Myr}$ ). We find 24 clusters in the younger+less massive bin, 7 clusters in the younger+more massive bin, 37 clusters in the older+less massive bin, and 34 clusters in the older+more massive bin. If we assume the younger bins to be correct, we can estimate from the older + more massive bin what we expect for the older+less massive bin: we would expect $\approx 117$ clusters to be in the older+less massive bin, which is roughly $13 \sigma$ away from the observed value, if one assumes purely Poissonian statistics. Secondly, we performed a KS-test to estimate the probability that the mass functions in both age bins are drawn from the same distribution. We find a probability of only 10 per cent if we adopt a lower mass cut-off at $\log$ (mass) $=3.2$ (to account for completeness effects), and a probability of only 14 per cent if we adopt a lower mass cut-off at $\log ($ mass $)=3.5$ (further reducing the completeness limit), hence both distributions are significantly different. Both tests show the significance of our results. There are several possible reasons for this change in the mass function with time.

One reason might be a relation between cluster masses and the available gas reservoir and the possible exhaustion of the gas reservoir by the first generation of star clusters in the burst, leaving only a small amount of gas available for the star clusters formed more recently. While this seems plausible, there is still a large amount of gas available. Israel (1988) estimated the amount of gas left in the galaxy to be mass $(\mathrm{HI})=1.1 \times 10^{8} \mathrm{M}_{\odot}$ and $\operatorname{mass}\left(\mathrm{H}_{2}\right)=2 \times 10^{7} \mathrm{M}_{\odot}$, while the total mass of the galaxy is estimated to be mass (total) $=3.3 \times 10^{8} \mathrm{M}_{\odot}$. Hence NGC 1569 is not gas-poor. It might still be a matter of the distribution of gas, and of the gas density. Taylor et al. (1999) studied the distribution of $\mathrm{CO}$ (and hence $\mathrm{H}_{2}$ ) in the centre of NGC 1569. They concluded that while there is a sufficient amount of $\mathrm{H}_{2}\left(\operatorname{mass}\left(\mathrm{H}_{2}\right) \simeq 7.7 \times 10^{6} \mathrm{M}_{\odot}\right)$, this mass is distributed over an area $\approx 200 \mathrm{pc}$ across, and hence not dense enough to form new clusters. The observed giant molecular clouds (GMCs), on the other hand, are not massive enough to form new SSCs assuming any reasonable overall star formation efficiency. It is noteworthy that the observed GMCs are not in the vicinity of the SSCs, but close to observed Hir regions, and hence near the most actively star-forming regions. The Hi maps of Stil \& Israel (2002) also indicate a depression of (neutral) gas near the SSCs (see also Greve et al. 2002), and a clumpy higher-density ridge (with intensity peaks East and West of the SSCs, $\approx 15^{\prime \prime}$ away from the SSCs, and thus just outside our FoV) along the galaxy's major axis, together with extended diffuse emission. The total amount of HI gas is estimated to be $\approx 1.3 \times 10^{8} \mathrm{M}_{\odot}$, but spread across a large area.

As suggested by the observations of Stil \& Israel (1998), the starburst in NGC 1569 might be triggered by the passage of a nearby Hi cloud (projected distance $\simeq 5 \mathrm{kpc}$ ) with mass $=7 \times 10^{6} \mathrm{M}_{\odot}$. There seems to be an Hi ridge 
connecting NGC 1569 to this companion (Stil \& Israel 1998, their fig. 3), supporting a scenario in which the HI cloud passed close to NGC 1569 in the recent past, on time-scales equivalent to the burst duration, i.e. of the order of few tens of Myr. The ram pressure compression of the interstellar medium (ISM) during the approach of the companion, and the absence of this compression during the time after perigalacticum, is another possible explanation for the change in the mass functions with time. This explanation is supported by observational (e.g. de Grijs et al. 2001, 2003b) and theoretical evidence (Ashman \& Zepf 2001, Elmegreen 2002) of enhanced average star cluster masses and star formation efficiencies caused by interaction-induced ram pressure.

A final possible, and in our opinion most likely, origin might be the strong radiation field caused by the large number of newly formed massive stars in the beginning of the burst and the follow-up energy input into the ISM by SNe. Waller (1991) and Origlia et al. (1998) estimate the number of SNe during the burst to be of order 2000-25,000 SNe/Myr. This might not only cause an unusually high dust temperature (34 K; e.g. Hunter et al. 1989, Lisenfeld et al. 2002) and the powering of the galaxy's strong thermal X-ray halo and bipolar outflow (Heckman et al. 1995, Della Ceca et al. 1996, Martin et al. 2002), but it might also prevent the assembly of larger molecular clouds due to heating of the ISM and pressure by UV photons and SN ejecta, since the collapse time-scale of a Jeans-instable cloud increases with its mass. Observed GMCs are likely to have collapsed while shock fronts, caused by the outflow of material, passed through high-density warm material (shock-cooling, see Taylor et al. 1999), and therefore driven by conditions not available in all regions of the galaxy.

\subsection{Summary}

We interpret multi-colour HST data of star clusters in the dwarf (post-)starburst galaxy NGC 1569 with dedicated evolutionary synthesis models and a robust analysis method to determine ages, metallicities, internal extinction values and masses of individual clusters independently. We conclude that we are observing a mainly young, recently formed, perhaps partially still forming, star cluster system, which mainly consists of objects considerably less massive than average globular clusters in the Milky Way. The extinction within NGC 1569 towards the clusters is found to be low, the metallicity distribution wide-spread, which is thought to be at least partially due to the age-metallicity degeneracy. These results are consistent with previous studies, but enlarge the sample of star clusters analysed in NGC 1569 by a factor of 4 .

We confirm the bursty character of star cluster formation in NGC 1569, with a major burst starting some $25 \mathrm{Myr}$ ago, possibly triggered by a passing $\mathrm{HI}$ cloud, and approximately continuous, low-level star cluster formation at earlier times. We detect a significant lack of high-mass clusters formed at the end of the burst, compared to clusters formed earlier in the burst. While the reason for this is still unclear, we consider three possibilities (or any interplay of them):

1. the absence of a sufficient amount of gas, or gas density, to form new (massive) star clusters;

2. the ram pressure caused by the companion Hi cloud is apparent predominantly during the companion's approach, and not during its recession; 
3. the heating of the ISM due to a strong radiation field and SN ejecta originating from the clusters formed in the beginning of the burst, preventing the assembly of massive molecular clouds by photo-ionisation and turbulence.

We believe all three scenarios to be of relevance, but attribute the highest importance to the latter one.

From a technical point of view we conclude that:

1. The commonly used procedure to assume a generic metallicity (and extinction) for all clusters is dangerous, since this affects the resulting age (and mass) distributions significantly.

2. With only optical passbands available the age-metallicity degeneracy largely precludes the determination of reliable ages and masses. While it is partially possible to correct for this, it introduces additional uncertainties. A more reliable way is by using additional NIR observations.

In the near future we will apply our methods to different star-forming environments, such as interacting galaxies of various types and in various stages of interaction. This will improve our understanding of the impact of the environment on the formation, evolution and disruption processes of recently formed star clusters, and their relation with old star clusters, like the well-studied globular clusters in the Milky Way.

\subsection{Acknowledgments}

This paper is based on archival observations with the NASA/ESA Hubble Space Telescope, obtained at the Space Telescope Science Institute, which is operated by the Association of Universities for Research in Astronomy (AURA), Inc., under NASA contract NAS 5-26555. This paper is also partially based on ASTROVIRTEL research support, a project funded by the European Commission under 5FP Contract HPRI-CT-1999-00081. This research has made use of NASA's Astrophysics Data System Abstract Service. This research has also made use of the NASA/IPAC Extragalactic Database (NED) which is operated by the Jet Propulsion Laboratory, California Institute of Technology, under contract with the National Aeronautics and Space Administration. PA is partially funded by DFG grant Fr 911/11-1. PA also acknowledges partial funding from the Marie Curie Fellowship programme EARASTARGAL "The Evolution of Stars and Galaxies", funded by the European Commission under 5FP contract HPMT-CT-2000-00132. We would like to thank Henny Lamers for many fruitful discussions and the hospitality of the University of Utrecht, as well as the anonymous referee for many useful suggestions. 


\section{Bibliography}

Aloisi A., Clampin M., Diolaiti E., Greggio L., Leitherer C., Nota A., Origlia L., Parmeggiani G., Tosi M., 2001, AJ, 121, 1425

Anders P., Fritze - v. Alvensleben U., 2003, A\&A, 401, 1063

Anders P., Bissantz N., Fritze - v. Alvensleben U., de Grijs R., 2004, MNRAS, 347,196

Arp H., Sandage A., 1985, AJ, 90, 1163

Ashman K. M., Conti A., Zepf S. E., 1995, AJ, 110, 1164

Ashman K. M., Zepf S. E., 2001, AJ, 122, 1888

Bertelli G., Bressan A., Chiosi C., Fagotto F., Nasi E., 1994, A\&AS, 106, 275

Boutloukos S. G., Lamers H. J. G. L. M., 2003, MNRAS, 338, 717

Buckalew B. A., Dufour R. J., Shopbell P. L., Walter D. K., 2000, AJ, 120, 2402

Calzetti D., Armus L., Bohlin R. C., Kinney A. L., Koornneef J., StorchiBergmann T., 2000, ApJ, 533, 682

Cerviño M., Valls-Gabaud D., Luridiana V., Mas-Hesse J. M., 2002, A\&A, 381, 51

Cerviño M., Valls-Gabaud D., 2003, MNRAS, 338, 481

Della Ceca R., Griffiths R. E., Heckman T. M., MacKenty J. W., 1996, ApJ, 469,662

Devost D., Roy J.-R., Drissen L., 1997, ApJ, 482, 765

Elmegreen B. G., 2002, ApJ, 577, 206

Eskridge P. B., Frogel, J. A., Taylor, V. A. et al., 2003, ApJ, 586, 923

Gerola H., Seiden P. E., 1978, ApJ, 223, 129

Gilbert A. M., Graham J. R., D., 2001, in Geisler D., Grebel E.K., Minniti D., eds, Proc. IAU Symp. 207, Extragalactic Star Clusters. Astron. Soc. Pac., San Francisco, p. 471 (GG01)

Girardi L., Bressan A., Bertelli G., Chiosi C., 2000, A\&AS, 141, 371 
González Delgado R. M., Leitherer C., Heckman T., Cerviño M., 1997, ApJ, 483, 705

Greggio L., Tosi M., Clampin M., de Marchi G., Leitherer C., Nota A., Sirianni M., 1998, ApJ, 504, 725

de Grijs R., Fritze - v. Alvensleben U., Anders P., Gallagher III J. S., Bastian N., Taylor V. A., Windhorst R. A., 2003a, MNRAS, 342, 259

de Grijs R., Anders P., Bastian N., Lynds R., Lamers H.J.G.L.M., O’Neil Jr. E.J., 2003b, MNRAS, 343, 1285

de Grijs R., Bastian N., Lamers H.J.G.L.M., 2003c, MNRAS, 340, 197

de Grijs R., Gilmore G. F., Johnson R. A., Mackey A. D., 2002a, MNRAS, 331, 245

de Grijs R., Gilmore G.F., Mackey A.D., Wilkinson M.I., Beaulieu S.F., Johnson R.A., Santiago B.X., 2002b, MNRAS, 337, 597

de Grijs R., O’Connell R. W., Gallagher III J. S., 2001, AJ, 121, 768

Greve A., Tarchi A., Hüttemeister S., de Grijs R., van der Hulst J.M., Garrington S.T., Neininger N., 2002, A\&A, 381, 825

Heckman T. M., Dahlem M., Lehnert M. D., Fabbiano G., Gilmore D., Waller W. H., 1995, ApJ, 448, 98

Ho L. C., Filippenko A. V., 1996, ApJ, 466, L83 (HF96)

Hodge P. W., 1974, ApJ, 191, L21

Hunter D. A., Thronson Jr. H. A., Casey S., Harper D. A., 1989, ApJ, 341, 697

Hunter D. A., O'Connell R. W., Gallagher III J. S., Smecker-Hane T. A., 2000, AJ, 120, 2383 (H00)

Israel F. P., 1988, A\&A, 194, 24

Izotov Y. I., Thuan T. X., Lipovetsky V. A., 1994, ApJ, 435, 647

Izotov Y. I., Thuan T. X., Lipovetsky V. A., 1997, ApJS, 108, 1

Izotov Y. I., Thuan T. X., 1998, ApJ, 500, 188

Kobulnicky H. A., Skillman E. D., 1997, ApJ, 489, 636

Kroupa P., Tout C. A., Gilmore G., 1993, MNRAS, 262, 545

Lejeune T., Cuisinier F., Buser R., 1997, A\&AS, 125, 229

Lejeune T., Cuisinier F., Buser R., 1998, A\&AS, 130, 65

Lisenfeld U., Israel F. P., Stil J. M., Sievers A., 2002, A\&A, 382, 860

Maoz D., Ho L. C., Sternberg A., 2001, ApJ, 554, L139 
de Marchi G., Clampin M., Greggio L., Leitherer C., Nota A., Tosi M., 1997, ApJ, 479, L27

Martin C. L., Kobulnicky H. A., Heckman T. M., 2002, ApJ, 574, 663

Origlia L., Leitherer C., Aloisi A., Greggio L., Tosi M., 2001, AJ, 122, 815

Schaerer D., de Koter A., 1997, A\&A, 322, 598

Schlegel D. J., Finkbeiner D. P., Davis M., 1998, ApJ, 500, 525

Schulz J., Fritze - v. Alvensleben U., Mller C. S., Fricke K. J., 2002, A\&A, 392, 1

Seiden P.E., Schulman L.S., Elmegreen B.G., 1984, ApJ, 282, 95

Smith L. J., Norris R. P. F., Crowther P. A., 2002, MNRAS, 337, 1309

Stasińska G., 1984, A\&AS, 55, 15

Stetson P. B., 1987, PASP, 99, 191

Stil J. M., Israel F. P., 1998, A\&A, 337, 64

Stil J. M., Israel F. P., 2002, A\&A, 392, 473

Taylor C. L., Hüttemeister S., Klein U., Greve A., 1999, A\&A, 349, 424

Tomita A., Ohta K., Saito M., 1994, PASJ, 46, 335

Vallenari A., Bomans D. J., 1996, A\&A, 313, 713

de Vaucouleurs G., de Vaucouleurs A., Pence W., 1974, ApJ, 194, L119

Waller W. H., 1991, ApJ, 370, 144

Whitmore B. C., Zhang Q., Leitherer C., Fall S. M., Schweizer F., Miller B. W., 1999, AJ, 118, 1551 


\section{Chapter 5}

\section{Photo press release on the NGC 1569 star cluster work}

The following release was prepared in close interaction with Lars Lindberg Christensen at ESO. It was released $3^{\text {rd }}$ February 2004. It can be found e.g. at http://www. spacetelescope.org/news/html/heic0402.html .

\section{Supernova blast bonanza in nearby galaxy}

The nearby dwarf galaxy NGC 1569 is a hotbed of vigorous star birth activity which blows huge bubbles and super-bubbles that riddle the main body of the galaxy. The galaxy's vigorous 'star factories' are also manufacturing brilliant blue star clusters. This galaxy had a sudden and relatively recent onset of star birth 25 million years ago, which subsided about the time the very earliest human ancestors appeared on Earth.

In this new image, taken with the NASA/ESA Hubble Space Telescope, the bubble structure is sculpted by the galactic super-winds and outflows caused by a colossal input of energy from collective supernova explosions that are linked with a massive episode of star birth.

Many open questions remain in astronomy as to how and when galaxies formed and how they evolved. Most of today's galaxies seem to have been already fully formed very early on in the history of the Universe (now corresponding to a large distance away from us), their formation involving one or more galaxy collisions and/or episodes of strongly enhanced star formation activity (so-called starbursts).

While most galaxies that are actually forming are too far away for detailed studies of their stellar populations even with Hubble, their local counterparts, nearby starburst and colliding galaxies, are far easier targets.

NGC 1569 is a particularly suitable example, being one of the closest starburst galaxies. It harbours two very prominent young, massive clusters plus a large number of smaller star clusters. The two young massive clusters match the globular star clusters we find in our own Milky Way galaxy, while the smaller ones are comparable with the less massive open clusters around us. 
NGC 1569 was recently investigated in great detail by a group of European astronomers who published their results in the January 2004 issue of the British journal, Monthly Notices of the Royal Astronomical Society. The group used several of Hubble's high-resolution instruments, with deep observations spanning a wide wavelength range to determine the parameters of the clusters more precisely than is currently possible from the ground.

The team found that the majority of clusters in NGC 1569 seem to have been produced in an energetic starburst that started around 25 million years ago and lasted for about 20 million years. First author Peter Anders from the Go"ttingen University Galaxy Evolution Group, Germany says "We are looking straight into the very creation processes of the stars and star clusters in this galaxy. The clusters themselves present us with a fossil record of NGC 1569's intense star formation history."

The bubble-like structures seen in this image are made of hydrogen gas that glows when hit by the fierce winds and radiation from hot young stars and is racked by supernovae shocks. The first supernovae blew up when the most massive stars reached the end of their lifetimes roughly 20-25 million years ago. The environment in NGC 1569 is still turbulent and the supernovae may not only deliver the gaseous raw material needed for the formation of further stars and star clusters, but also actually trigger their birth in the tortured swirls of gas.

The colour image is composed of 4 different exposures with Hubble's Wide Field and Planetary Camera 2 through the following filters: shown in blue a wide ultraviolet filter ( 800 seconds), in green a green filter (930 seconds), in red a wide red filter (550 seconds) and also in red a Hydrogen alpha filter (1600 seconds).

\section{Notes for editors:}

The team is composed of Peter Anders (Go"ttingen University Galaxy Evolution Group, Germany), Richard de Grijs (University of Sheffield, UK), and Uta Fritze - v. Alvensleben (Go"ttingen University Galaxy Evolution Group, Germany).

This composite image was constructed with data from the ESO/ST-ECF Science Archive. The original Hubble exposures were obtained by Hunter (Proposal 6423).

Animations of the discovery and general Hubble Space Telescope background footage are available from:

http://www.spacetelescope.org/bin/ videos.pl?searchtype=news\&string=heic0402

Image credit: European Space Agency, NASA \& Peter Anders (Göttingen University Galaxy Evolution Group, Germany)

The Hubble Space Telescope is a project of international cooperation between ESA and NASA. 


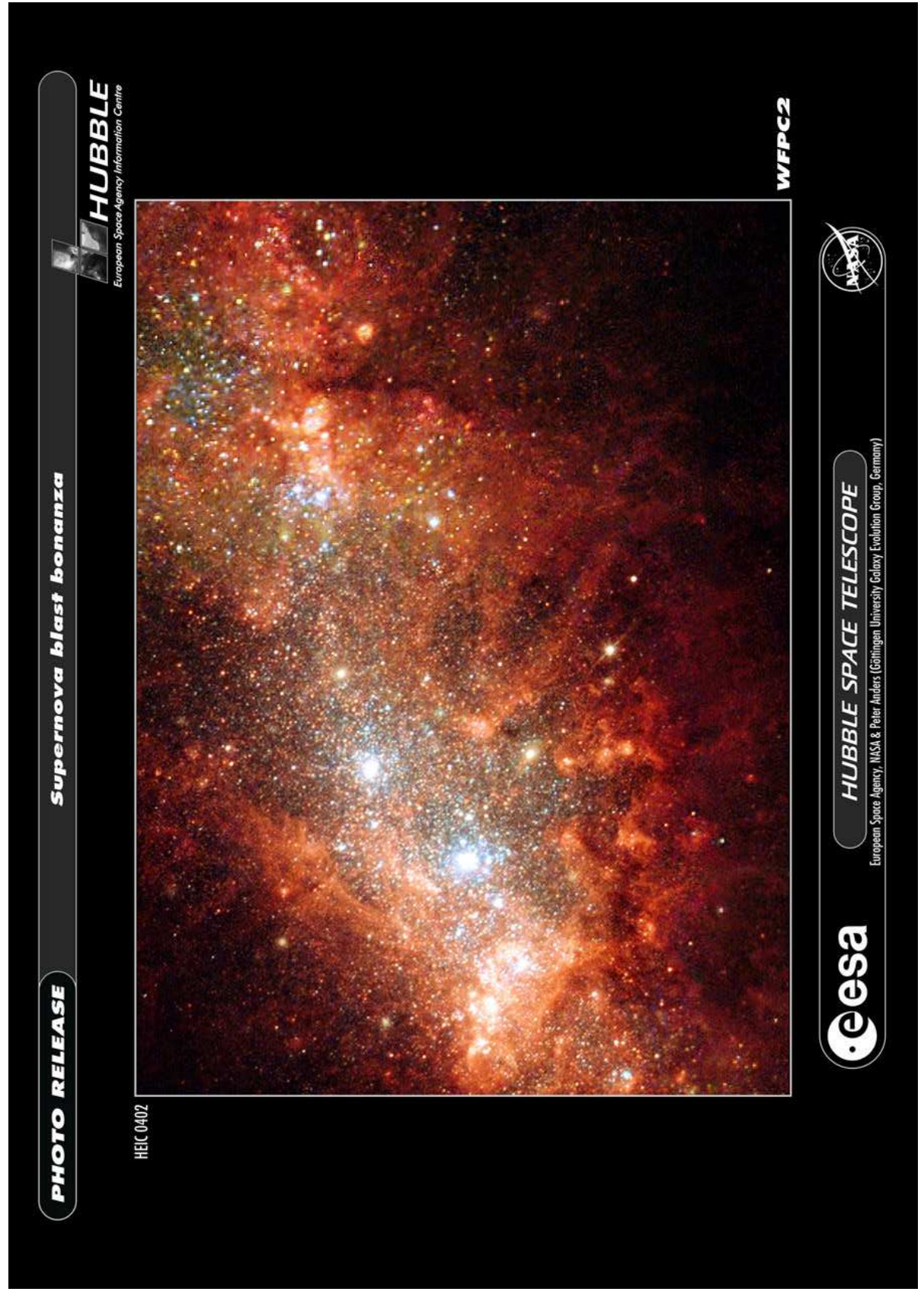

Figure 5.1: Photo press release on the star clusters in NGC 1569 



\section{Chapter 6}

\section{Accurate photometry of extended spherically symmetric sources ${ }^{1}$}

\subsection{Introduction}

We present a new method to determine photometric properties of extended spherically symmetric sources in Hubble Space Telescope (HST) data obtained with the Wide Field and Planetary Camera 2 (WFPC2), the Advanced Camera for Surveys / Wide Field Camera (ACS/WFC), and camera 2 of the Near Infrared Camera and Multi Object Spectrometer (NICMOS).

When studying extragalactic star clusters (SCs) at high spatial resolution, such as with the HST, the accuracy of "classical" photometric methods becomes insufficient. Ideally, fitting the point-spread functions (PSFs) is desirable for sources in crowded fields and with variable background fluxes. However, this is difficult since SCs at distances of $\lesssim 20 \mathrm{Mpc}$ appear extended on the HST images and, as a consequence, PSF fitting techniques will underestimate their true fluxes.

With the best spatial resolution possible to achieve today $(\sim 0.05$ arcsec with the HST, namely using WFPC2/PC, ACS/WFC and ACS/HRC) many nearby clusters are clearly resolved. We define a "clearly resolved" cluster conservatively as having $1.2 \times$ the PSF size, and hence an observed cluster FWHM roughly of the order of 2.3 pixels (see Table 6.3 ). As will be shown below, these 2.3 pixel correspond to an intrinsic cluster FWHM on the order of 0.5 pixel.

In addition, the high spatial resolution of the WFPC2 and ACS cameras undersample the PSF. For marginally extended sources, a satisfactory solution to this undersampling problem has recently been included in the HSTPHOT PSF fitting software package custom-written to handle HST photometry (Dolphin 2000).

Measuring the light in a fixed annulus around the central source coordinates, as commonly done in aperture photometry, can in principle correct for both the undersampled PSF and source size. However, when studying a population of

\footnotetext{
${ }^{1}$ This chapter has been accepted for publication in A\&A as Anders, Gieles \& de Grijs 2006
} 
sources with variable sizes, as for extragalactic SC systems in general, using a fixed aperture will underestimate the flux of the larger sources with respect to that of the point-like sources.

Many extragalactic SC studies have tried to estimate the size of the sources based on the magnitude difference in different apertures (we will refer to this as the "DeltaMag method"), and compare these to either model clusters (usually assuming Gaussian light profiles; e.g. Whitmore et al. 1993; Whitmore \& Schweizer 1995; Zepf et al. 1999) and/or observed star profiles (e.g. Zepf et al. 1999). Sometimes, multiple apertures and cumulative light distributions are used, thus enhancing reliability (e.g. Puzia et al. 1999). However, as shown in de Grijs et al. (2001), the presence of a variable, structured background strongly compromises the results from the DeltaMag method.

In the same studies, estimates of aperture corrections (ACs) needed to account for the finite size of the objects are given, again on the basis of either model clusters (e.g. Whitmore \& Schweizer 1995) or isolated clusters in the science images of interest (e.g. Miller et al. 1997; Carlson et al. 1998), mostly determined for a subset of clusters and applied to the whole sample - independent of object size. Some authors do attempt to use size-dependent ACs (e.g. Zepf et al. 1999), although generally not well defined, and mostly based on the rough size estimates resulting from the magnitude difference method. This method is vulnerable to centering problems (the use of 0.5 pixel radius apertures is seen regularly), and the sizes (and derived size-dependent ACs, as a consequence) are only rough estimates.

Other studies are based on more subjective methods, such as those that determine the source and sky annuli for each cluster individually, to encircle the dominant cluster light contribution and to avoid background contamination (see e.g. de Grijs et al. 2001; Anders et al. 2004). While this method avoids ACs (since it is already supposed to measure the dominant light contribution), it is hampered by subjectivity, and does not provide reliable size estimates.

There exist, as yet, no large-scale theoretical studies of the reliability, reproducibility and comparability of the results for any of these methods. All are subject to subjectivity in one aspect or another (e.g. the choice of apertures for size estimates/photometry, cluster light profile, selection of a few single clusters to derive "average" ACs).

To date, only two sophisticated systematic studies have been done to determine accurate SC sizes:

- Carlson \& Holtzman (2001), but limited to marginally resolved, high S/N sources, without studying the accompanying ACs

- Dolphin \& Kennicutt (2002) related to the above-mentioned program package HSTphot and its application to (again) marginally resolved sources in NGC 3627. This study is based on a PSF-fitting strategy for extended sources, while our work is based on aperture photometry.

The present study complements, expands upon and enhances those of Carlson \& Holtzman (2001) and Dolphin \& Kennicutt (2002). This study also fully complements structural studies of resolved clusters, e.g. in the Large and Small Magellanic Clouds (LMC, SMC) and nearby dwarf galaxies (see e.g. Mackey \& Gilmore 2003a,b). However, such studies are only possible for the very nearest galaxies and their clusters. Where ACs are concerned, this study extends the 
widely-used work of Holtzman et al. (1995) for point sources to the studies of extended spherically symmetric sources.

In this paper, we present a new method to perform more accurate aperture photometry of extended spherically symmetric sources using a simple extension to the basic principle of aperture photometry. After measuring the flux of each source using a fixed aperture size, a variable AC (based on the actual size of the object) is applied. This method greatly enhances reproducibility and comparability of the results obtained. With the large range of parameter space explored and numerous related effects taken into account, we also present for the first time a method to estimate uncertainties in the sizes and ACs for a given observation.

In Section 6.2 we propose a general definition of "size", as a function of a large number of intrinsic and observational parameters. In Section 6.3 the relation between source FWHM and the appropriate AC is determined as a function of aperture size. In Section 6.4 we provide a detailed "cookbook", ready for immediate application to extragalactic SC systems. The reader who is only interested in applying our ACs could skip directly to Section 6.4. In Section 6.5 we provide an example error analysis for our new method, including a comparison of the method presented here to the DeltaMag method.

\subsection{Determining accurate source sizes}

Conventionally, the stellar density distributions of old globular clusters (GCs) are well described by King profiles (King 1962) with a range of concentration parameters. Intermediate-age and young star clusters (YSCs), e.g., in the LMC, are better described by Elson, Fall \& Freeman (1987; EFF) profiles. Such clusters, similar to YSCs in, e.g., the Antennae galaxies or NGC 7252, do not (yet) show evidence of tidal truncations, in contrast to King profiles. We set out to analyse SC systems containing SCs spanning a large range of ages, masses and sizes, and compare radii and compactnesses of SCs in different galaxies. We therefore need a reliable method to estimate, to high accuracy, the radii of a large variety of SCs.

Thus, we first have to find a general definition of "size". To this end, we created artificial SCs based on a variety of profiles using the BAOLAB package of Larsen (1999) (for recent applications see Larsen 2004a; Boeker et al. 2004). BAOLAB creates artificial clusters of a given magnitude by randomly drawing the position of each recorded photon from the input light profile. It thus simulates the stochastic nature of real observations very effectively, indeed more effectively than any other program available. These profiles were convolved with pre-calculated PSFs, generated with the TINY TIM package (Krist $\&$ Hook 2004), and (for WFPC2 and ACS/WFC) the appropriate diffusion kernels supplied by TinY Tim. Although some caveats still exist, TinY TIM is the best suited package to obtain realistic HST PSFs to date. First, it extensively covers the parameter space of interest (cameras, filters, chips, position on the chips, object spectra, focus, PSF sizes etc.), well beyond anything that can be realisticly done with observed PSFs. And secondly, and even more crucial, the subsampling of TINY TIM PSFs allows one to study the real distribution of counts onto adjacent pixels, depending on the exact PSF peak position on subpixel basis. This subsampling is fully implemented and used in the BAOLAB 
package.

In order to measure the size of these objects we fit Gaussian profiles to them. Many extragalactic SC systems observed to date display a wide range of cluster sizes, so that we need to have a consistent and robust size determination to compare SC sizes and compactnesses. Therefore, we decided to apply a blanket fitting approach of Gaussian profiles to the SC light distributions. We realise that this is a simplification, but fitting more complicated profiles (such as King or EFF profiles) requires high signal-to-noise $(\mathrm{S} / \mathrm{N})$ ratios and the knowledge of whether or not the clusters are tidally truncated. In practice, this will be difficult for a large number of sources in realistic SC samples. We point out that the actual, underlying cluster profile is only of minor importance for the relative size determinations of SCs in a given SC system; the key prerogative is that one applies a consistent approach to one's size determinations. Since we also base our ACs on such Gaussian fits our approach is fully internally consistent, and we have, in effect, taken the detailed profile shape out of the equation. In Sections 6.2.1 to 6.2.7 we will perform a detailed analysis for the WFPC2 camera. In Section 6.2.8 and 6.2.9 we will expand this to the ACS/WFC and NICMOS/NIC2 cameras.

Finally, we note that fitting more realistic light profiles results in less stable fit results, since either King or EFF profiles have one additional free parameter (concentration and power-law slope, respectively). They are also more sensitive to features at the periphery of the cluster, i.e., in the low-S/N regions.

Throughout this paper, we will use the FWHM of the input profile and the measured FWHM of the fitted Gaussian profile as measures for the size. In Table 6.1 we present the (constant) conversion factors from FWHM to the more widely used half-light radius, $R_{1 / 2}$, for the different models (e.g., Larsen 2004b).

\subsubsection{The parameters of the "standard" cluster}

In the following subsections we will investigate the behaviour of the measured cluster sizes (using the FWHM of a Gaussian profile, as justified in the previous section) as a function of the input FWHM, assuming various parameters for the artificial clusters and a range of observational conditions. In Section 6.8.1 we provide conversion relations between input and measured FWHM (and vice versa), by fitting fifth-order polynomials to these conversion relations, of the form

$$
\operatorname{size}(x)=a+b * x+c * x^{2}+d * x^{3}+e * x^{4}+f * x^{5}
$$

and

$$
\operatorname{size}^{\prime}(y)=a^{\prime}+b^{\prime} * y+c^{\prime} * y^{2}+d^{\prime} * y^{3}+e^{\prime} * y^{4}+f^{\prime} * y^{5}
$$

where $x$ and $\operatorname{size}^{\prime}(y)$ are the intrinsic FWHM in pixels, and size $(x)$ and $y$ the measured FWHM. We decided to use fifth-order polynomials after a visual inspection of the data and the fit results, as a compromise between fitting details in the shape, wiggles in the fits, and usability. We note that this choice is purely based on mathematical convenience, and not on any physical properties of the SCs. 
We first define our "standard" cluster:

- "Observations": cluster of $\operatorname{mag}(\mathrm{WFPC} 2 / \mathrm{F} 555 \mathrm{~W} \approx V)=10 \mathrm{mag}$, observed in a 1s exposure;

- Tiny Tim PSF properties: HST WFPC2/WF3 chip; central position on the chip, i.e., $(x, y)=(400,400)$; F555W filter; using standard WFPC2 diffusion kernel;

- BAOLAB parameters: no noise, profile fitting radius $=5$ pixels; used for cluster generation and cluster fitting;

- Input cluster light profiles: Gaussian; King (1962) models with concentrations $c=5,30,100^{2}$; Elson et al. (1987) models with power-law index $\gamma=1.5,2.5$ (cf. Section 6.2.2);

- Fit model: Two-dimensional (2D) Gaussian profile, without taking into account any TINY TIM HST PSF or HST diffusion kernel (effectively using a delta function-type PSF).

Although in the following sections we will plot the conversion relations for a larger range of input FWHMs (in order to illustrate that we understand their behaviour across the entire range of realistic sizes), we strongly advise to use these relations only in the range of $0.5 \leq$ input FWHM (pixels) $\leq 10$. For smaller input FWHM, the data are not well approximated by the fitted polynomials. For larger input FWHMs, the $\mathrm{S} / \mathrm{N}$ ratio per pixel decreases, and as a consequence the noise increases, so that the fit will not be sufficiently accurate. In other words, after converting measured radii to "intrinsic" radii, it is advisable to treat clusters with "intrinsic" radii outside the 0.5 - 10 pixel range with caution.

\subsubsection{Size determination as a function of input model}

In this Section we use the "standard" clusters defined in Section 6.2.1. The models used are a Gaussian model, King (1962) models with $c=5,30$ and 100 (King 5, King 30 and King 100, respectively), and EFF profiles (Elson et al. 1987) with power-law indices $\gamma=1.5$ and 2.5 (EFF 15 and EFF 25, respectively). We point out that the power-law index $\gamma$ used in BAOLAB differs by a factor of 2 from the definition used by Elson et al. (1987), with $\gamma_{\mathrm{EFF}}=2 \times \gamma_{\mathrm{BAOlab}}$.

The light profiles are represented by the following equations, where $r$ is the (dimensionless) radius (in units of FWHM), and $w$ is a (dimensionless) normalisation constant:

- Gaussian:

$$
f(r)=\exp \left(-(r \cdot w)^{2}\right)
$$

$$
\text { with } w=2 \sqrt{\ln (2)} \approx 1.66
$$

\footnotetext{
${ }^{2}$ The concentration parameter, $c$, is the ratio of tidal to core radius, $c \equiv r_{t} / r_{c}$; note that the concentration is more often given as $\log (c)$
} 
Table 6.1: Conversion factors to calculate a model's $R_{\mathrm{eff}}=R_{1 / 2}$ from its FWHM.

\begin{tabular}{|c|c|}
\hline model & $R_{1 / 2} /$ FWHM \\
\hline GAUSS & 0.5 \\
King 5 & 0.71 \\
King 30 & 1.48 \\
King 100 & 2.56 \\
EFF 15 & 1.13 \\
EFF 25 & 0.68 \\
\hline
\end{tabular}

- King models:

$$
f(r, c)=\left(\frac{1}{\sqrt{1+(r \cdot w)^{2}}}-\frac{1}{\sqrt{1+c^{2}}}\right)^{2},
$$

with $w=2 \sqrt{\left(\sqrt{0.5}+\frac{1-\sqrt{0.5}}{\sqrt{1+c^{2}}}\right)^{-2}-1}$, so that $w \approx 1.69,1.95$ and 1.98 for King 5, King 30 and King 100, respectively.

- EFF models:

$$
f(r, \gamma)=\left(1+(r \cdot w)^{2}\right)^{-\gamma},
$$

with $w=2 \sqrt{2^{1 / \gamma}-1}$, i.e., $w \approx 1.53$ and 1.13 for EFF 15 and EFF 25 , respectively.

\section{Effect of the PSF on a Gaussian profile}

The first step is to assess what the effect of PSF "blurring" is on Gaussian profiles. Standard clusters (see Section 6.2.1) were created using Gaussian input profiles with different FWHMs. Subsequently, 2D Gaussian profiles were fit to the resulting images. A Gaussian fit results in either a Gaussian width, $\sigma$, where FWHM $=2 \sqrt{2 \ln (2)} \sigma$, or directly in the FWHM, in most commonly used Gaussian fitting routines.

Results for a range of input FWHM values from 0.1 to 15 WF3 pixels are shown in Fig. 6.1. The offset caused by the convolution of the input profile with the instrumental PSF decreases with increasing input cluster size, since the PSF and diffusion kernel broadening become less and less important. For clusters with input FWHMs greater than $\sim 3$ pixels, the relation between input FWHM and recovered FWHM of the Gaussian fit is approximately linear, and the derived ("measured") cluster sizes are of the order of $0.3-0.6$ pixels (3-20 per cent) larger than the input ("intrinsic") values.

For clusters with input FWHMs greater than $\sim 10$ pixels, the scatter increases because of the low $\mathrm{S} / \mathrm{N}$ ratio per pixel.

To conclude, we understand the general behaviour of this data set very well. However, since the Gaussian cluster light profile is the least realistic input 

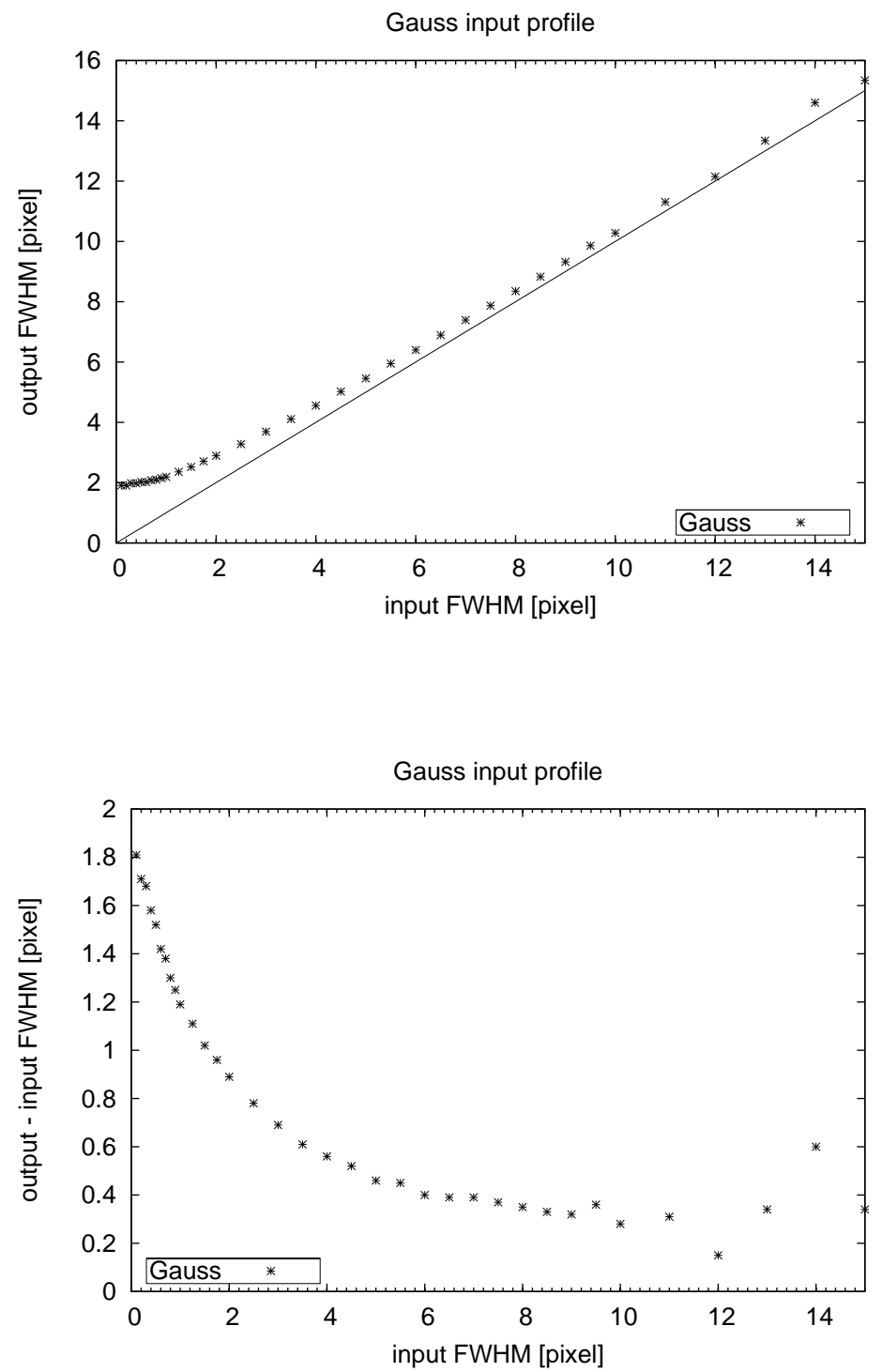

Figure 6.1: Fitted Gaussian FWHMs for input Gaussian profile convolved with WFPC2/WF3 F555W PSF, located on the central pixel. The diagonal solid line in the top panel represents a one-to-one relation. Top: Output FWHM. Bottom: Output - input FWHM.

profile, we will not consider it in the remainder of this study. In this section we simply wanted to demonstrate that the method works in a comprehensible way. In the following sections we will use more realistic input light profiles.

\section{Non-Gaussian input models}

For a given FWHM, the less concentrated King/EFF model profiles have less light in the wings than more concentrated King/EFF profiles, while a com- 


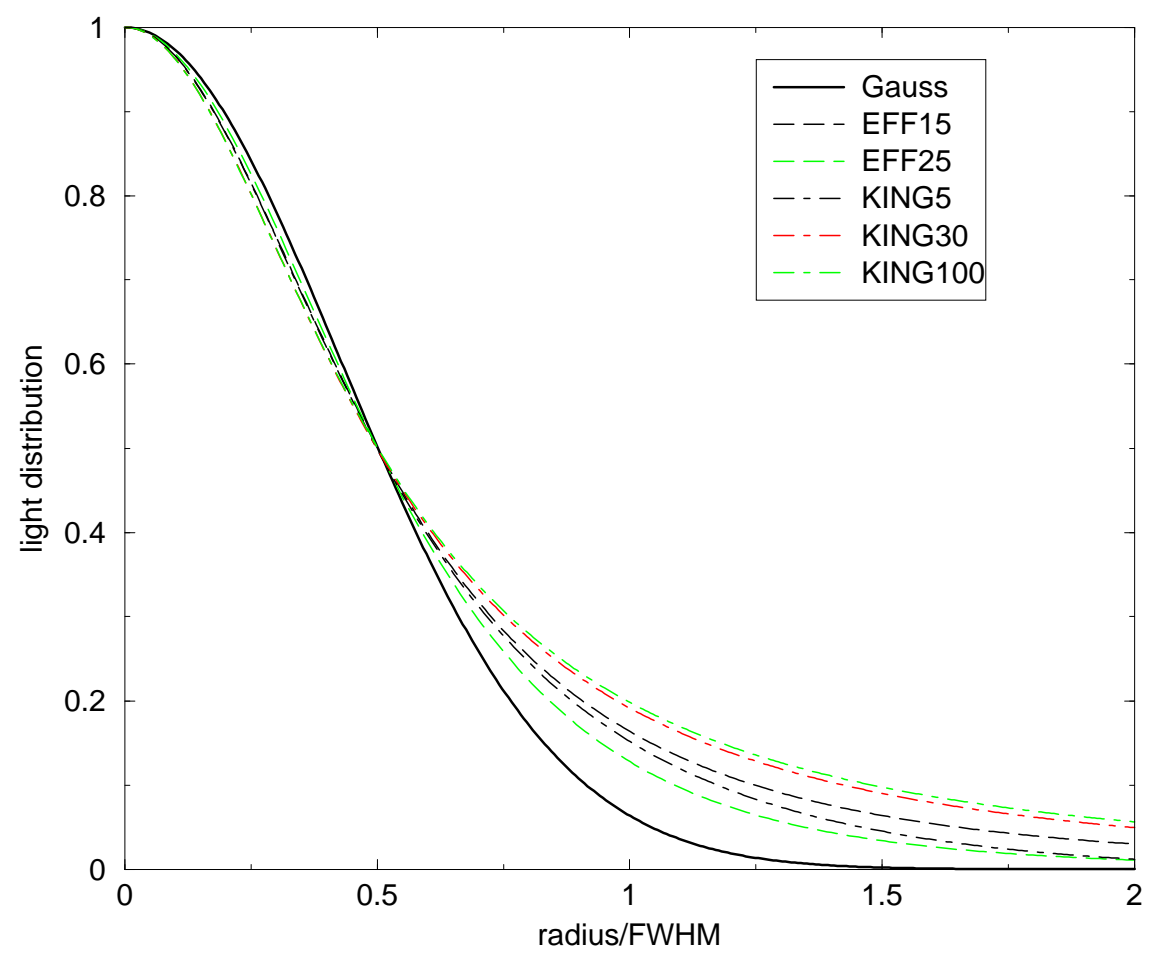

Figure 6.2: Model light distributions.

parable Gaussian profile has least flux in the wings. At radii smaller than the FWHM, non-Gaussian models appear somewhat more compact than a Gaussian profile. This is illustrated in Fig. 6.2.

In the first part of this study we adopt a fixed fitting radius of 5 pixels. We made this conscious choice for the fitting radius because for any realistic extragalactic SC observed with the HST at a decent S/N ratio, profile fits using Gaussian profiles are generally feasible. Larger fitting radii may be unproportionally affected by non-Gaussianity in the cluster profiles, low-S/N regions (i.e., fluctuations in the background noise), or neighbouring objects in crowded regions; much smaller fitting radii may not always be appropriate to employ Gaussian profile fits. To illustrate this, in Section 6.2.4, we will show that changing the fitting radius leads to systematic changes (and even numerical instabilities) of the ACs, and explain why this is the case.

Combining the choice of our 5-pixel fitting radius and the general behaviour of our input models, we expect to see the following trends:

- For clusters with input FWHM greater than 5 pixels, only the inner core will be fit. Due to the greater compactness of non-Gaussian models with respect to Gaussian profiles, for a given FWHM, we expect to systematically underestimate the sizes of large clusters.

- For clusters with FWHM smaller than 5 pixels, (i) the impact of PSF/diffusion kernel blurring of the cluster profile is more important, and (ii) the fit also 
includes the wings, which are more extended for non-Gaussian than for Gaussian profiles with identical FWHM. Therefore, we expect the sizes of small clusters to be overestimated.

- The 5-pixel boundary adopted was estimated from the size and shape of the cluster light profile alone. The application of PSF and diffusion kernel do not only change the size, but also the shape of the cluster profile. This causes unpredictable shifts in this empirical boundary. Nevertheless, we emphasise that the fit residuals are very small, as we will show below.

Since King models with large concentration indices and EFF models with small power-law index deviate most significantly from Gaussian profiles, we expect the largest deviations from a one-to-one relation between input and output FWHM for such models.

\section{King profiles}

For King profiles the results of this exercise are shown in Fig. 6.3. As expected, we find that for the less concentrated King-profile clusters, the relation between input and output FWHM deviates most from the relation for a Gaussian input model, i.e., from a strict one-to-one relation. The differences between King 5 and King 100 profiles reach $\sim 1$ pixel, with the King 5 results lying closer to the one-to-one relation.

\section{EFF profiles}

For young clusters in the LMC, which do not show any signs of tidal truncation, the best fit to the light distribution is a power law (Elson et al. 1987).

Fig. 6.4 shows the relation between input EFF-model FWHM and the FWHM of the Gaussian fit. The same systematic underestimate of large cluster sizes using Gaussian fits is observed as for the King models in the previous section, as expected. The differences between EFF 15 and EFF 25 profiles reach $\sim 1$ pixel, with the EFF 25 profiles lying closer to the one-to-one relation.

\section{Fitting using the respective input profiles}

To disentangle the effects of assuming a Gaussian profile (instead of the assumed input profile) on one hand and of the PSF/diffusion kernel on the other we ran a set of simulations using the input profile as fitting profile (instead of a Gaussian). The results presented in Fig. 6.5 indicate that the strong non-linearity seen in Figs. 6.3 and 6.4 originate from using the Gaussian fitting profile instead of the "correct" (input) profile. Using the input profile as fitting profile causes only a general offset (broadening of the light profile due to the PSF and the diffusion kernel). Unfortunately, the use of EFF/King profiles for light profile fitting is not implemented as standard even in some uptodate image reduction software packages, while a Gaussian is (to our best knowledge). Therefore, while sticking to the generally applicable Gaussian fitting profile we point at the origin of the non-linearities of our results. 

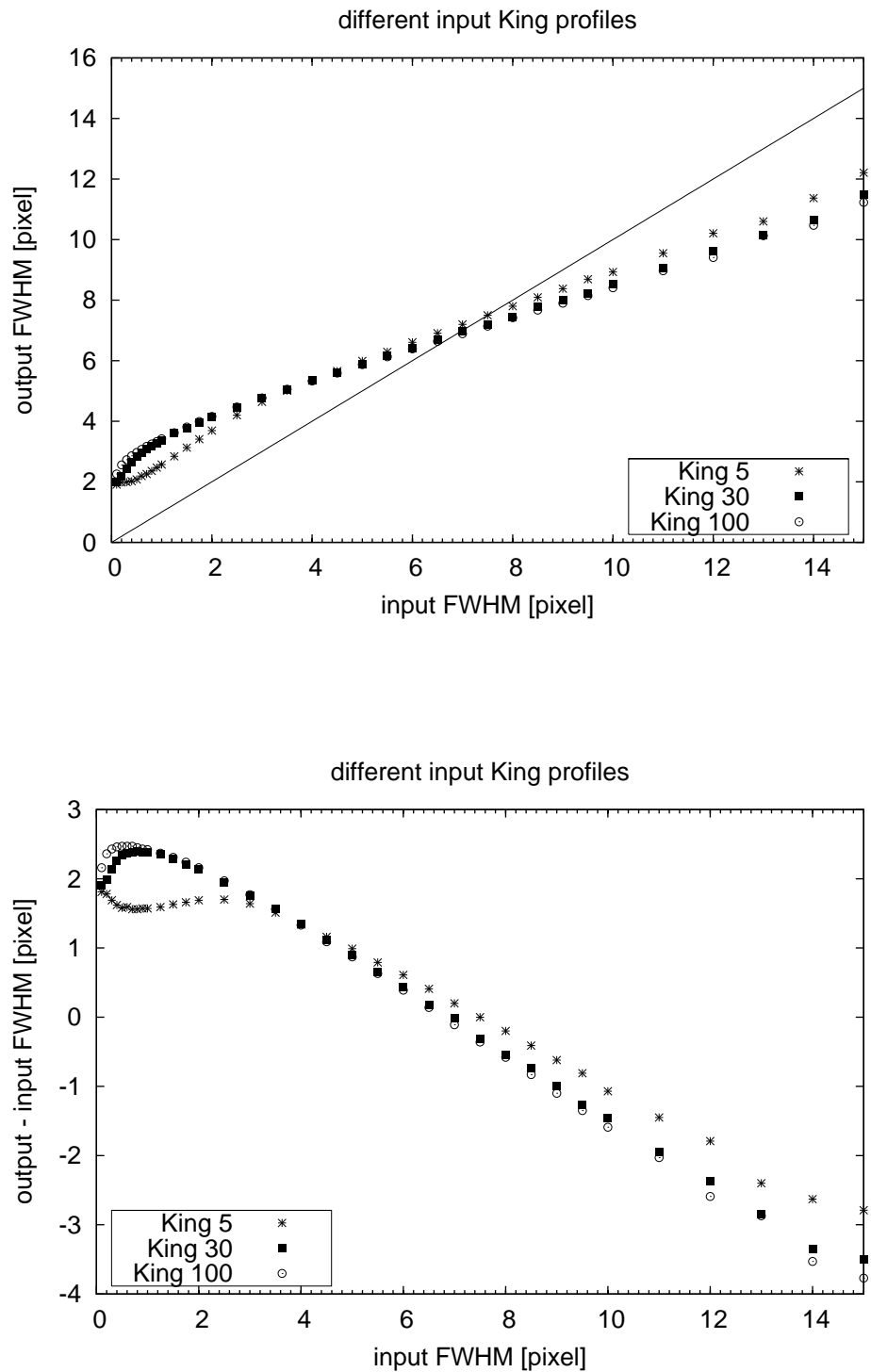

Figure 6.3: Fitted Gaussian FWHM for input King profiles with different concentrations: $*, c=5 ; \mathbf{\square}, c=30 ; \odot, c=100$. The diagonal solid line represents a one-to-one relation.

\section{Presentation of the fit results}

We fit the relation between the input FWHM of various profiles and the output FWHM of the Gaussian fits using a fifth-order polynomial function. The conversion relations that relate the input to the output FWHM, and vice versa, are presented in Section 6.8.1. The latter relation is most important to deduce the intrinsic size of a source from the measured size.

The whole list of tables includes the tables for the ACs (as will be determined in Sect. 6.3) in Sections 6.8.2 - 6.8.3.

In the following subsections, we will show the results for the two physically 

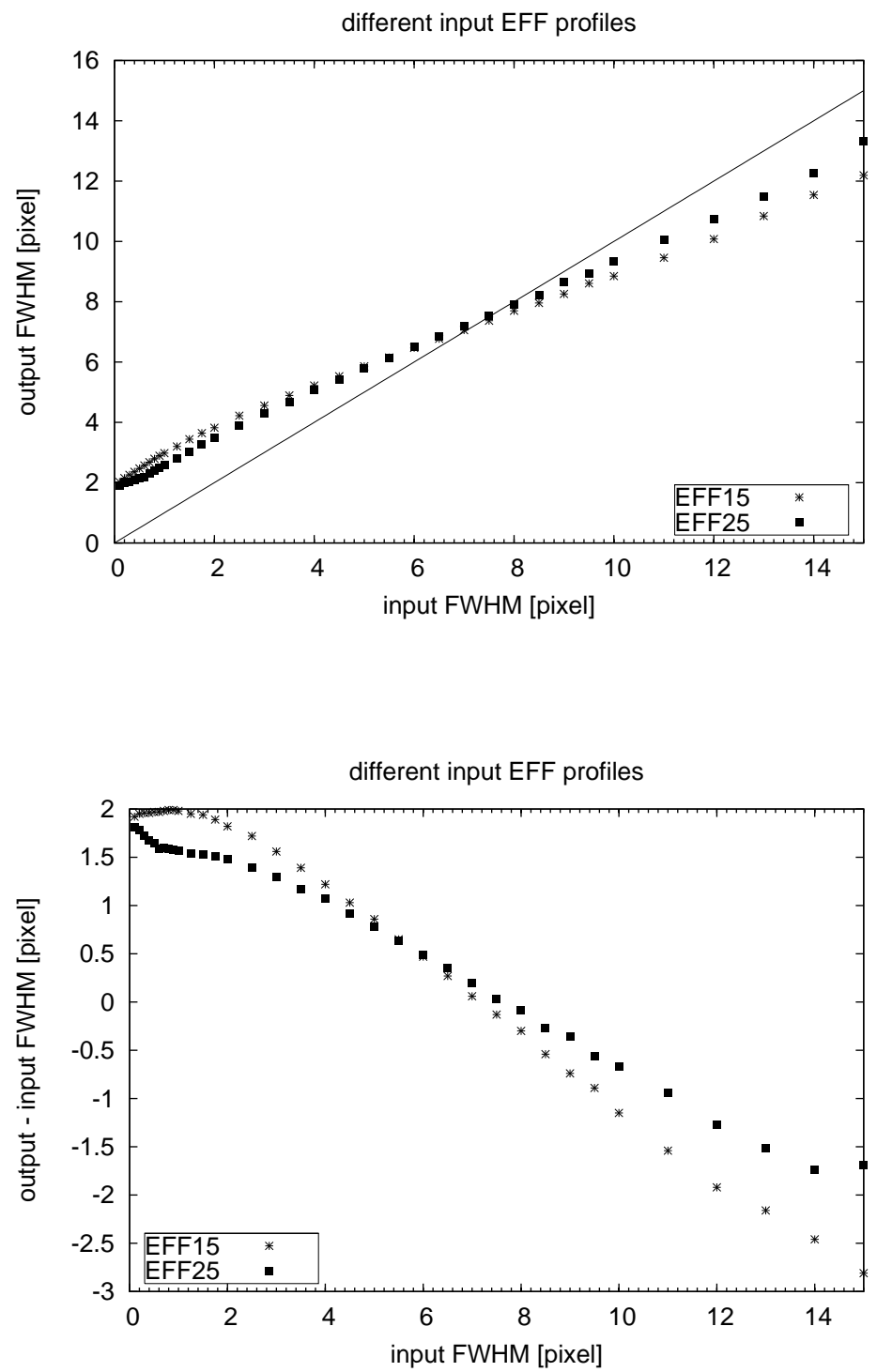

Figure 6.4: Fitted Gaussian FWHM for input EFF profiles with different powerlaw indices: $*, \gamma=1.5 ; \boldsymbol{\square}, \gamma=2.5$. The diagnonal solid line represents a one-to-one relation.

most interesting input models only, the King 30 and the EFF 15 models. These represent the average cluster light profiles of old Milky Way GCs (e.g., Binney \& Tremaine 1998) and YSCs in the LMC (Elson et al. 1987), respectively. Although the realistic light profiles differ significantly from a Gaussian profile, Figs. 6.3 and 6.4 show that the fits to our conversion relations are very accurate. For input FWHM $>0.5$ pixel the deviations of the fits from the data are always smaller than 4 per cent, while for smaller FWHM it might be as large as 10 per cent. The conversion functions for the full set of input models are given in Section 6.8.1. 

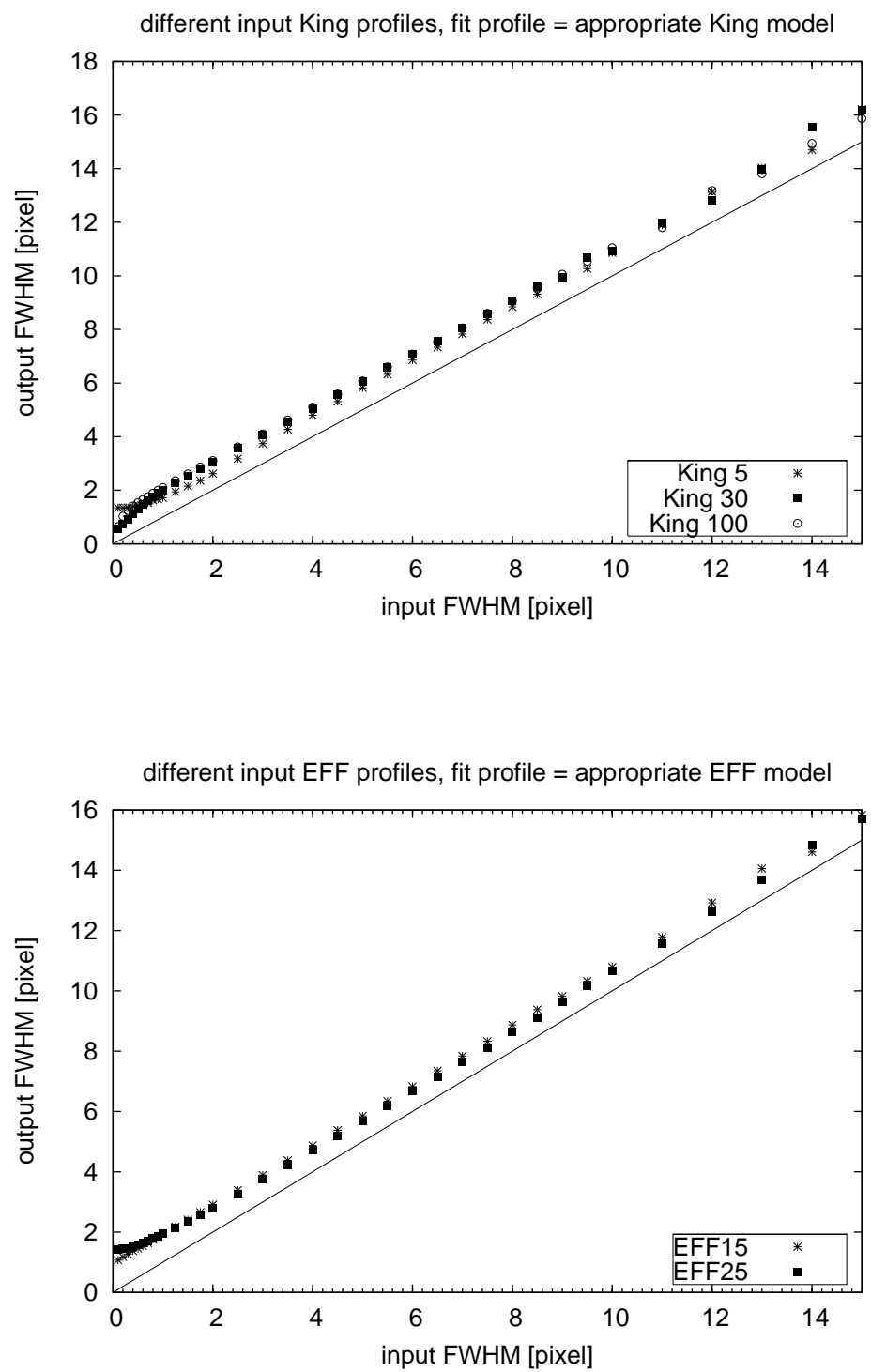

Figure 6.5: Fitting standard artificial clusters with different input profiles, using the input profile shape as fitting profiles (instead of the Gaussian). The diagnonal solid line represents a one-to-one relation. Top: Different King profiles. Bottom: Different EFF profiles.

All data are also available in electronic form from our website, at http://www.astro.physik. uni-goettingen.de/ galev/panders/Sizes_AC/ - This public dataset does not only include the parameters of the fitted conversion functions, but also the averaged data used for the fitting to allow for customized fit functions/interpolations etc. 


\subsubsection{Effect of cluster brightness: Fits and fit errors}

Thus far, we considered bright, noiseless artificial clusters of a given magnitude $(V=10 \mathrm{mag})$, "observed" in a 1s exposure.

We performed a series of simulations, varying the cluster magnitudes from $V=8 \mathrm{mag}$ to $V=14 \mathrm{mag}$. The results are shown in Figs. 6.6 and 6.7. For each magnitude and cluster profile, the results from 40 independent runs were averaged to reduce the scatter. The data from the individual runs, including the associated $1 \sigma$ uncertainties are compiled in Figs. 6.8 and 6.9 to show the amount of scatter.

Clearly, the conversion relations depend only weakly on the magnitude of the cluster. Deviations arise because the scatter in the relation increases with decreasing $\mathrm{S} / \mathrm{N}$ ratio per pixel, as e.g. caused by decreasing cluster brightness and/or increasing cluster sizes. In addition, for clusters with sauch low $\mathrm{S} / \mathrm{N}$ ratios per pixel, the readout noise might have some impact. See Sect. 6.2.5 for further details.

When we compare the results from Figs. 6.6 and 6.7 to those of Figs. 6.8 and 6.9 , we see that the scatter/deviations in the former figures for fainter clusters is fully consistent with the intrinsic scatter caused by the random nature of the algorithm used to generate the artificial clusters, even for averaged results. For bright clusters (represented by the $V=10$ mag cluster), the random scatter is on the order of \pm 0.1 pixel in the FWHM (for clusters larger than about 8 pixels FWHM up to \pm 0.2 pixel in the FWHM, and up to \pm 1 pixel for clusters larger than 11 pixels FWHM). This scatter increases with decreasing cluster brightness (up to $\pm 0.4-0.5$ pixels in the FWHM for a $V=14$ mag cluster smaller than about 7 pixels FWHM, and up to \pm 3 pixels in the FWHM for clusters larger than 7 pixels FWHM). The increasing scatter for large clusters is caused by the lower $\mathrm{S} / \mathrm{N}$ ratio per pixel. The data are all presented in terms of the individual absolute values from the different runs to illustrate the scatter. The median value scatters significantly less.

In summary, for the average cluster, the conversion relations for bright clusters can be applied to clusters of all magnitudes: For fainter clusters and at larger radii (hence for cases with low $\mathrm{S} / \mathrm{N}$ ratios per pixel) the cluster-to-cluster variations get larger, but scatter symmetrically around the average conversion relation.

\subsubsection{Fitting radius variations}

BAOLAB has the advantage that the fitting radius can be adjusted easily. In fact, the choice of fitting radius has a major impact on the cluster sizes that one determines, as we will show in this section. We performed tests using fitting radii in the range from 3 to 15 pixels (larger and smaller fitting radii did not lead to any meaningful results owing to numerical problems related to the convergence of the size fitting). As one can see from Figs. 6.10 and 6.11, the larger the fitting radius one adopts, the larger the apparent cluster radius one measures, and the stronger the deviations from the input values become. In fact, increasing the fitting radius seems to result in continuously increasing recovered cluster radii. This is caused by the impact of (i) the intrinsic profile mismatch between King and EFF profiles, and (ii) the PSFs/diffusion kernels and their non-Gaussianity. The fitting radius dependencies of the results will 

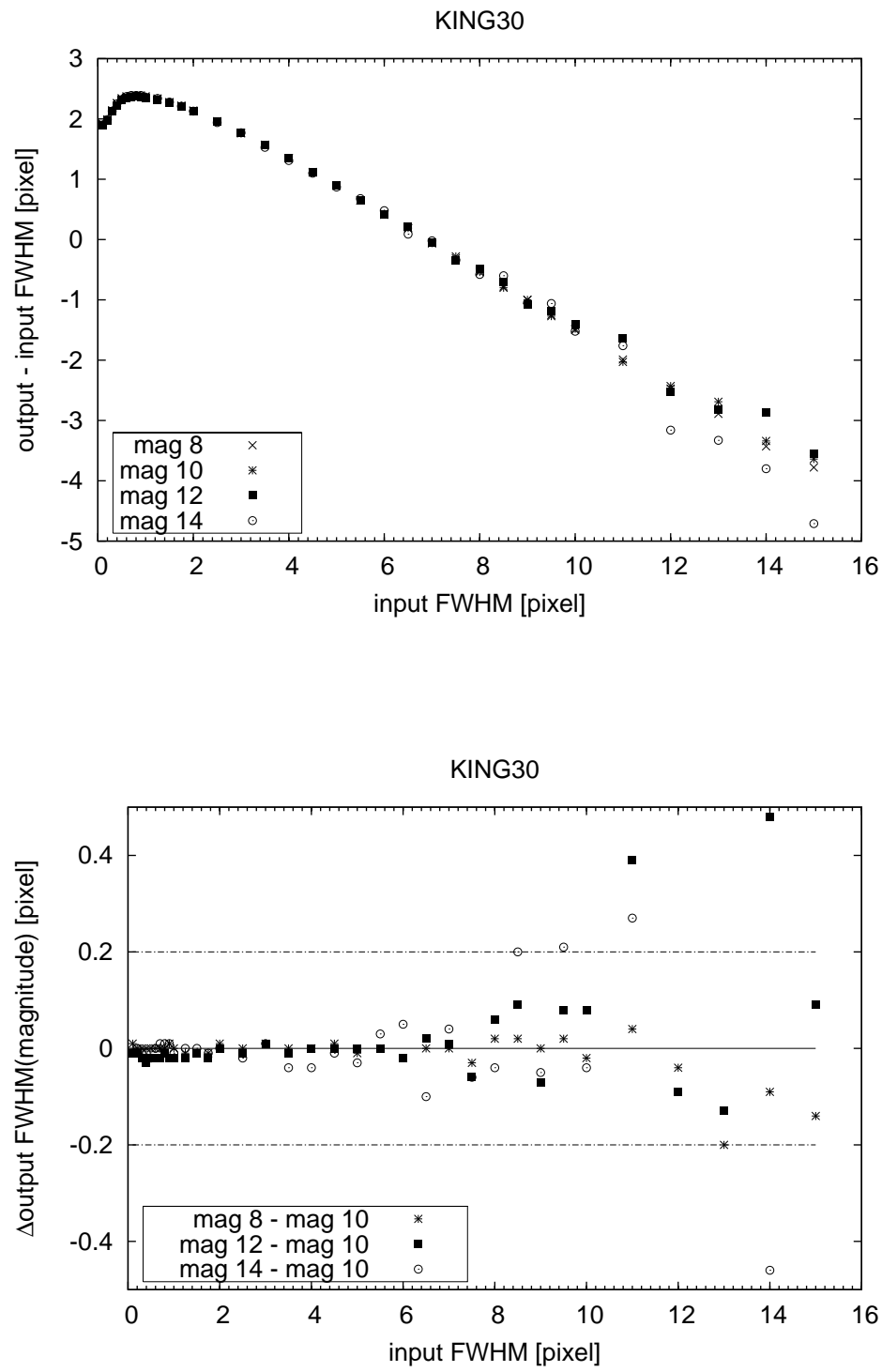

Figure 6.6: Fitted Gaussian FWHM for input King 30 profiles of different cluster magnitudes. Top: Output - input sizes, simulated data and fitted polynomials. Bottom: Comparison of fit functions, using the $V=10 \mathrm{mag}$ fit function as reference.

be significantly lower if one were to fit the clusters with the correct cluster light profile, including the right PSF and diffusion kernel. However, since we wanted to keep our study as generally applicable as possible, we did not make use of the respective functions BAOLAB provides in the standard settings. However, we refer the reader to Section 6.2.6, where we discuss this in more detail.

As shown by Carlson \& Holtzman (2001), even fitting King profiles (which are thought to be more realistic, at least for old globular clusters) to observed cluster profiles is fitting radius dependent. They attribute this behaviour to 

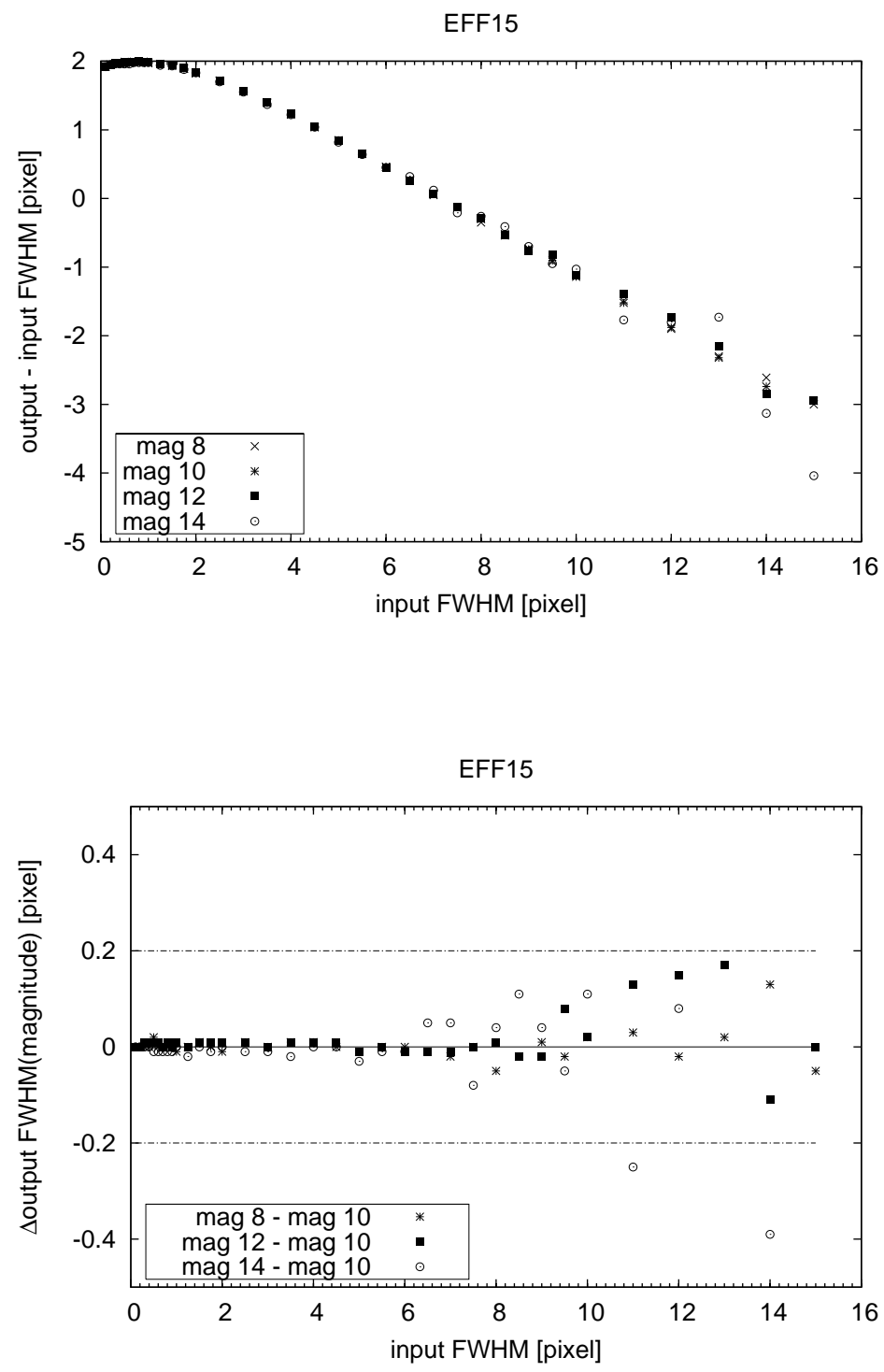

Figure 6.7: Fitted Gaussian FWHM for input EFF 15 profiles of different cluster magnitudes. Top: Output - input sizes, simulated data and fitted polynomials. Bottom: Comparison of fit functions, using the $V=10$ mag fit function as reference.

inaccuracies of the PSFs at small radii. And indeed, their situation is different from ours, in the sense that in our case we expect the intrinsic differences of the input profiles and the fitted Gaussian to dominate the fitting behaviour, not inaccuracies of the PSFs, while for Carlson \& Holtzman (2001) the profile mismatch, if any, is likely smaller.

A selection of fit residuals is included in Section 6.8.4, in Figs. 6.34 and 6.35, as a function of fitting radius and input cluster radius. The area shown covers the inner $5 \times 5$ pixels. For fitting radii $<5$ pixels, the solution tends to become 

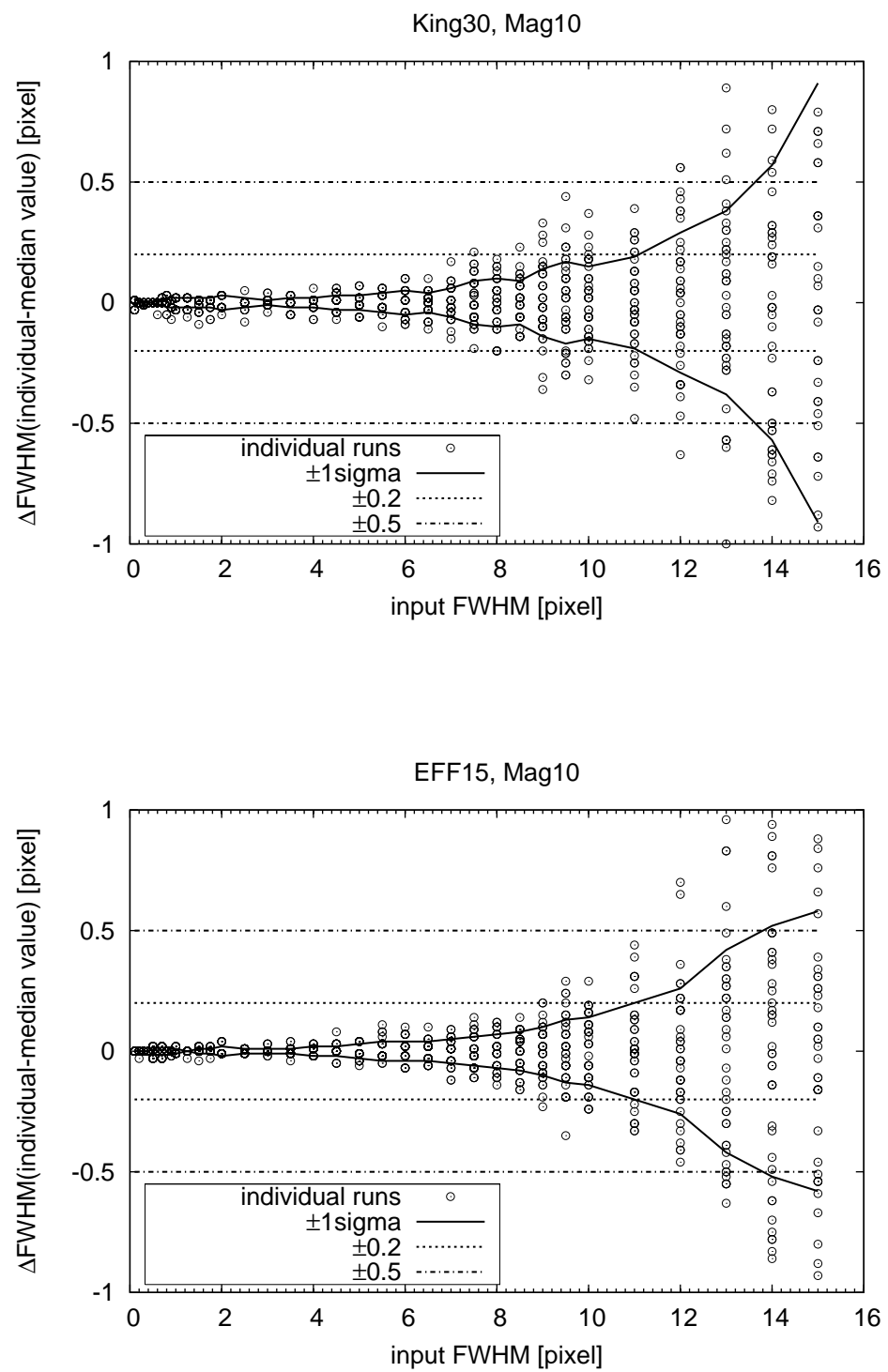

Figure 6.8: Comparison for $V=10$ mag King 30 (upper panel) and EFF 15 (lower panel) clusters. Horizontal lines indicate \pm 0.2 pixels and \pm 0.5 pixels. The solid curved lines indicate the $\pm 1 \sigma$ range of the scatter. Shown are the differences of the individual runs with respect to the average value.

computationally unstable, as shown in Fig. 6.10.

For small clusters, the residuals shown in Figs. 6.34 and 6.35 are almost independent of the fitting radius, because in all cases the cluster is much smaller than the fitting radius. However, the residuals are significantly non-negligible, clearly showing the intrinsic difference in shape between Gaussian and King/EFF profiles.

For large clusters (we show the results for clusters with FWHMs of 5.0 and 10.0 pixels, respectively), the residuals are relatively small for small fitting radii 

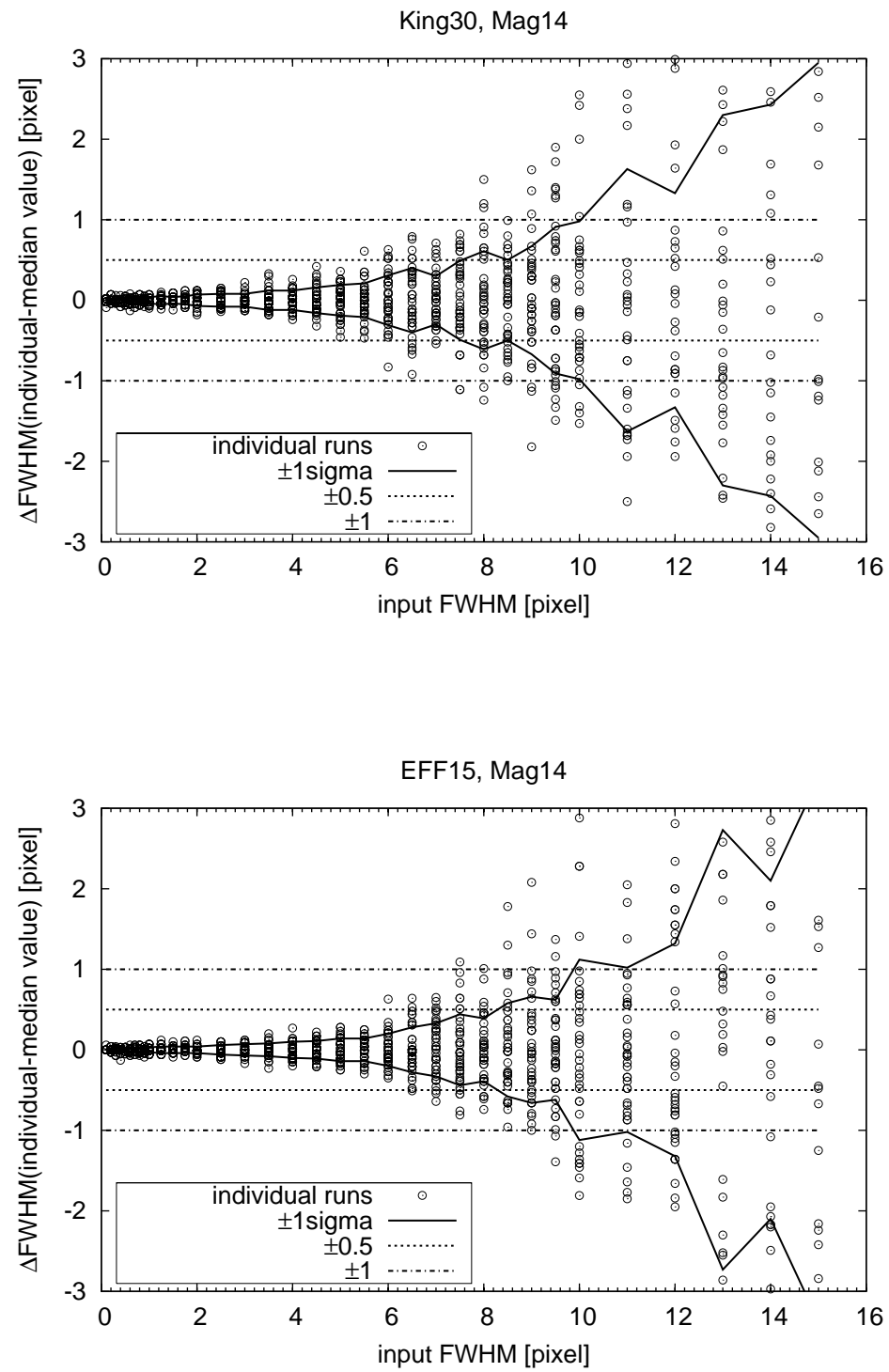

Figure 6.9: Comparison for $V=14$ mag King 30 (upper panel) and EFF 15 (lower panel) clusters. Horizontal lines indicate \pm 0.5 and \pm 1.0 pixels. The solid curved lines indicate the $\pm 1 \sigma$ range of the scatter. Shown are the differences of the individual runs with respect to the average value.

(e.g., fitting radii on the order of the input FWHM), where the fit is dominated by the inner parts of the clusters, which resemble Gaussian profiles. For fitting radii greater than the FWHM, the cluster wings are given too much weight, resulting in strong deviations in the inner cluster parts and large residuals (just as for "small" clusters, discussed above). The maximum residuals increase by a factor 3-5 for fitting radii from 5 to 15 pixels. However, fitting the inner cluster parts only seems to be more promising for 2 reasons: (i) the inner cluster region resembles a Gaussian profile more closely, and hence fitting with a Gaussian 
function is less problematic, and (ii) the $\mathrm{S} / \mathrm{N}$ ratio per pixel is higher in the inner parts than in the wings.

In summary, one would like to have a fitting radius large enough to give stable results (larger than 3 pixels, cf. Figs. 6.10 and 6.11), but small enough to fit mainly the cluster core rather than the wings, to avoid serious problems with structures in the immediate environment of the cluster (e.g., variable background, crowding effects, etc.) and to produce (close to) negligible deviations from the input size. In addition, as shown in Figs. 6.10 and 6.11, the impact of changing the fitting radius is such systematic and significant, that a single, generic value for the fitting radius is needed. Otherwise, the entire analysis in this paper must be done for each individual data set.

We therefore recommend the use of a generic fitting radius of 5 pixels (which should be applicable to almost all realistic observations), and emphasise that all results given in this paper were thus obtained.

\section{Origin of the strong fitting radius dependence of the results}

The cause of the strong fitting radius dependence of our size conversion relations most likely also causes the non-linearity of the size conversion relations, i.e., the shape difference between the intrinsic cluster profile (EFF or King profiles) and the Gaussian used for the fitting.

To test this hypothesis we have performed a set of simulations similar to the ones in the previous section, except now the fit models are the same as the input models, and they were convolved internally with the appropriate PSF and the diffusion kernel. The results, shown in Fig. 6.12, partially support our hypothesis, even though for large fitting and cluster radii the behaviour is still non-linear, and differs systematically among the fitting radii.

We conclude that in order to get a one-to-one correlation between input and output FWHM the fitting radius must be at least larger than the cluster radius. In case the fitting radius equals the cluster radius, the deviations from a oneto-one correlation are typically of the order of $-0.2 /-0.3$ pixel, as can be seen in Fig. 6.12. However, these deviations/non-linearities are intrinsically taken into account in our results.

\subsubsection{Impact of the sky background}

In this section we assess the importance of the sky background. We model $V=10 \mathrm{mag}$ and $V=14$ mag clusters, each with background levels of $0,1,3,5$, $10,20,30,50$, and 100 ADU ("counts") per pixel. We use 20 independent runs, and show the averaged results in Figs. 6.13 and 6.14, and the associated plots illustrating the actual scatter in Figs. 6.15 and 6.16 for the selected magnitudes, $V=10$ and $V=14 \mathrm{mag}$, respectively.

Since sky noise and readout noise have the same characteristics, this Section combines both effects.

On average, the results seem to be robust with respect to (constant) changes in the sky background. There is only a slight tendency for faint clusters on a strong sky background to appear marginally smaller (see Fig. 6.14).

The impact of (Poissonian) shot noise from the cluster itself is negligible.

In order to allow for a rough estimate of the $\mathrm{S} / \mathrm{N}$ ratios for the clusters and background levels discussed in this Section we provide the approximate count 


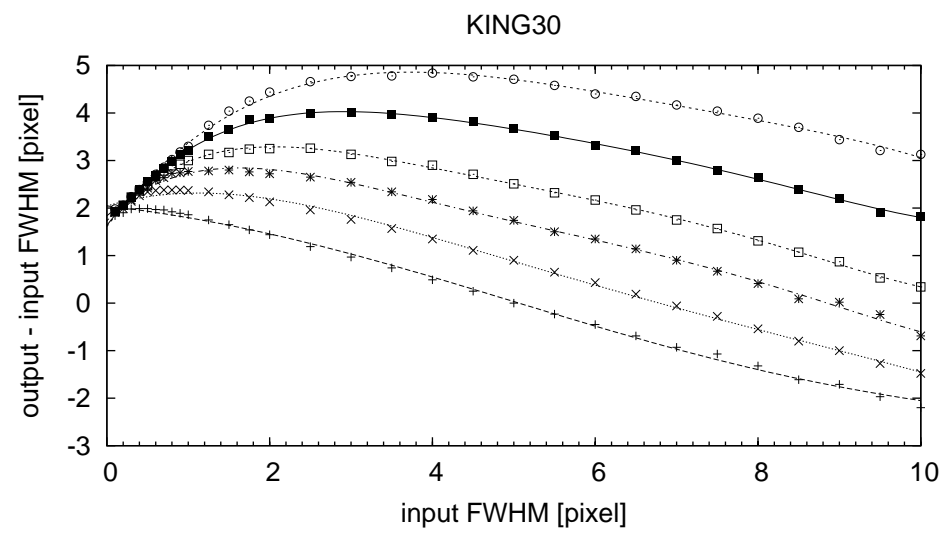

\begin{tabular}{|c|c|c|c|}
\hline $\begin{array}{r}\text { data FITRAD }=3 \\
\text { fit FITRAD }=3 \\
\text { data FITRAD }=5\end{array}$ & $\begin{array}{c}+ \\
+- \\
\times\end{array}$ & $\begin{array}{r}\text { data FITRAD }=7 \\
\text { fit FITRAD }=7 \\
\text { data FITRAD }=9\end{array}$ & a \\
\hline
\end{tabular}

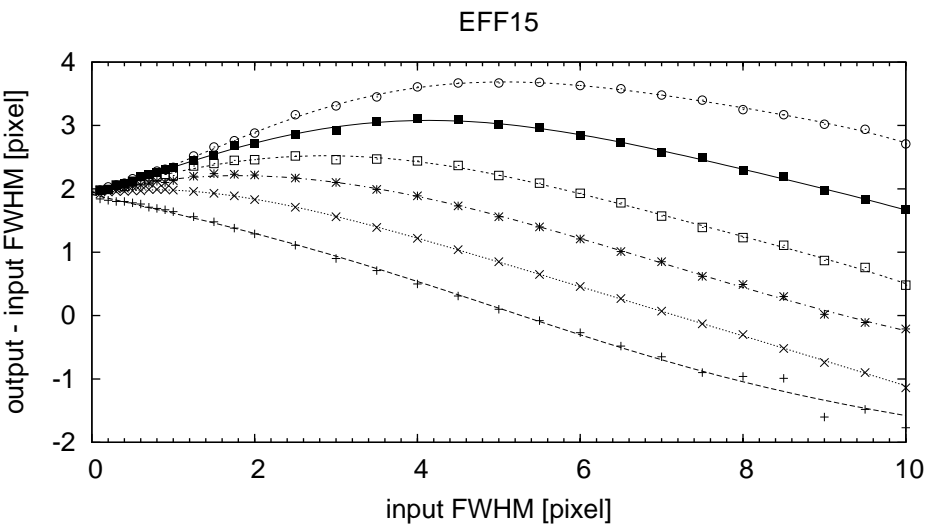

\begin{tabular}{|c|c|c|c|c|c|}
\hline $\begin{aligned} \text { data FITRAD } & =3 \\
\text { fit FITRAD } & =3 \\
\text { data FITRAD } & =5 \\
\text { fit FITRAD } & =5\end{aligned}$ & $\begin{array}{c}+ \\
+ \\
\times\end{array}$ & $\begin{array}{r}\text { data FITRAD }=7 \\
\text { fit FITRAD }=7 \\
\text { data FITRAD }=9 \\
\text { fit FITRAD }=9\end{array}$ & \begin{tabular}{c}
$*$ \\
$\cdots$ \\
\hdashline$\cdots$
\end{tabular} & $\begin{array}{r}\text { data FITRAD }=12 \\
\text { fit FITRAD }=12 \\
\text { data FITRAD }=15 \\
\text { fit FITRAD }=15\end{array}$ & - \\
\hline
\end{tabular}

Figure 6.10: Conversion relations for a standard cluster, using different fitting radii (given in pixel units in the legend).

rates in the peak pixel of selected clusters in Table 6.2.

\subsubsection{Using the appropriate PSFs for fitting}

For four HST/WFPC2 filters we checked to what extent the filter-dependent PSFs affect the cluster size determinations. BAOLAB allows one to consider the appropriate PSF when fitting the cluster size by convolving the Gaussian model clusters with the PSF specified. Hence we created clusters with PSFs for the HST/WFPC2 $U, B, V, I$-band equivalent filters; while fitting the size of the cluster, BAOLAB took the appropriate PSF into account. Here, we investigate the impact and possibilities of this approach. 


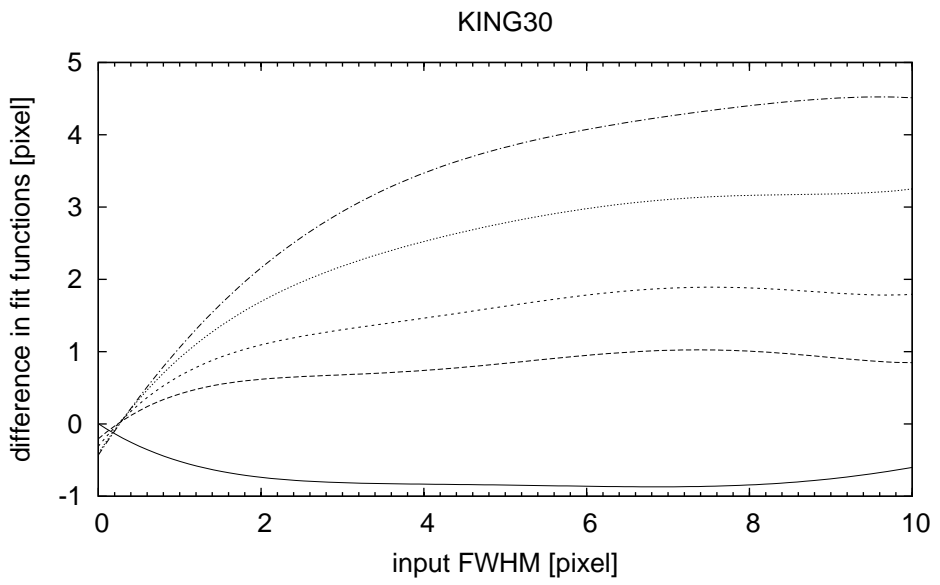

\begin{tabular}{lrr} 
FITRAD $3-5-$ & FITRAD $9-5 \cdots \cdots$ \\
FITRAD $7-5$ & FITRAD $12-5 \cdots$ & FITRAD 15 - $5 \cdots$ \\
\hline
\end{tabular}

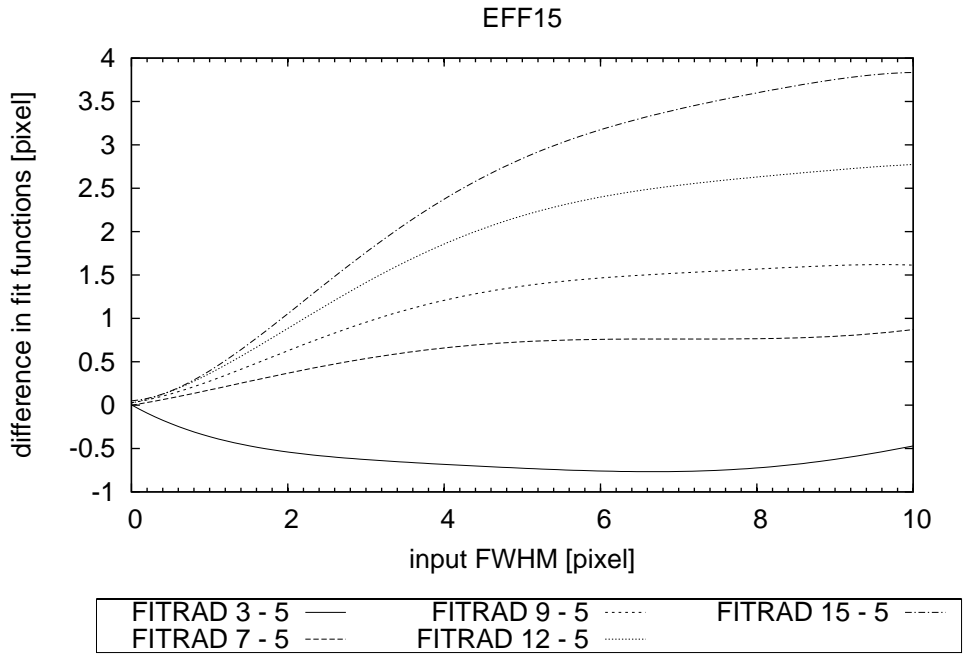

Figure 6.11: Conversion relations for a standard cluster, using different fitting radii (given in pixel units in the legend). Displayed are the offsets of these relations from the relation for a fitting radius of 5 pixels.

The results are presented in Fig. 6.17. While the relations appear to be slightly noisier (despite the use of 40 independent runs), the differences between the different filters are well within the "normal" scatter of \pm 0.2 pixel.

\subsubsection{Other dependences}

We investigated the results for different WFPC2 filters and chips. Both variations lead to only minor differences in the results for the "standard" cluster. However, for completeness we give the results in Tables $6.5-6.8$. 

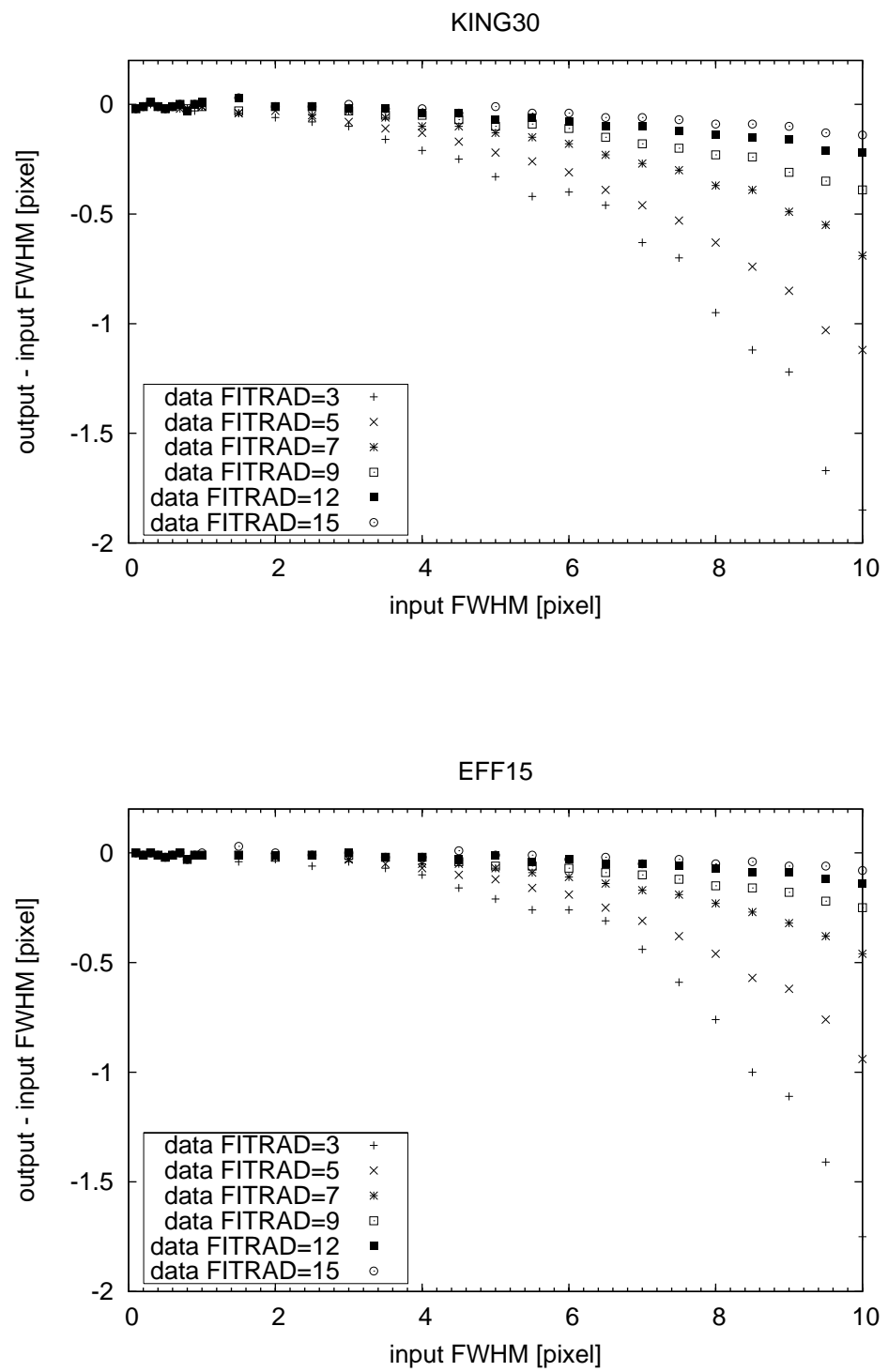

Figure 6.12: Conversion relations for a standard cluster, using different fitting radii (given in pixel units in the legend) and taking the input light profile, PSF and diffusion kernel into account.

We also checked the dependence of the results on the position of the artificial cluster on the chip, and on subpixel shifts. Both tests gave results within the "standard" random scatter of \pm 0.2 pixel.

In addition, we produced a number of PSFs assuming various spectral types for the standard cluster, ranging from O5 to M3. Again, all differences remain within the usual random scatter of \pm 0.2 pixel.

These "non-dependencies" are consistent with Carlson \& Holtzman (2001), who found that different PSFs only have a minor impact. 
Table 6.2: Count rates [in ADU] in the peak pixel of selected clusters, to approximate $\mathrm{S} / \mathrm{N}$ ratios for the background levels

\begin{tabular}{|c|c|c|c|c|}
\hline $\begin{array}{c}\text { cluster's FWHM } \\
\text { [pixel] }\end{array}$ & $\begin{array}{c}\text { King 30 } \\
V=10\end{array}$ & $\begin{array}{c}\text { EFF 15 } \\
V=10\end{array}$ & $\begin{array}{c}\text { King 30 } \\
V=14\end{array}$ & $\begin{array}{c}\text { EFF 15 } \\
V=14\end{array}$ \\
\hline 1.0 & 6200 & 7600 & 150 & 180 \\
2.0 & 3100 & 4100 & 80 & 100 \\
5.0 & 900 & 1200 & 25 & 30 \\
10.0 & 300 & 400 & 8 & 10 \\
\hline
\end{tabular}

\subsubsection{Observing with ACS: chip, position, and filter de- pendence}

Since both of the HST ACS/WFC chips (WFC1 and WFC2) are located well off the instrument's optical axis, the PSFs suffer from severe geometrical distortions and the diffusion kernel is both wavelength and position dependent. Therefore, we carried out simulations with TINY TIM PSFs for both WFC chips, for various positions on the chip, and - for the central positions of each camera - also using different filters (F435W, F555W, and F814W, roughly equivalent to BessellJohnson-Cousins $B, V$ and $I$ ). Again, 40 independent runs were used to obtain average values with reduced scatter.

The results for the different filters used with the ACS/WFC are shown in Fig. 6.18. The strongest differences are observed for the F814W filter, for which the sizes we determined are systematically larger, by $\sim 0.1-0.2$ pixels, than those obtained for the F555W filter. The reason is not quite clear, as both the PSF and the diffusion kernel appear less extended than the respective values for the F555W filter. In addition, Fig. 6.18 shows prominent discontinuities for the F814W filter around input FHWMs of 0.7 pixel. These peaks are statistically significant, and apparent in almost every single run.

The differences in the $\mathrm{F} 435 \mathrm{~W}$ filter are significantly smaller. In addition, when comparing the results for the $\mathrm{F} 555 \mathrm{~W}$ passband for the two WFC chips, we find only small differences. The deviations in the latter two cases are below or on the order of \pm 0.05 pixel.

Despite the distortion of the chips and the corresponding changes in the PSF with position across the chip, we find only a small impact on the derived sizes of the cluster position on the chips. For almost all clusters the deviations are well within \pm 0.1 pixel. Hence, for almost all purposes the central PSF (and the associated diffusion kernel) can be used.

\subsubsection{Observing with NICMOS: filter-dependence}

PSF construction for NICMOS using TINY TIM is not straightforward, mainly because of the off-focus setting during early observations [i.e., before servicing mission 3B]. This caused strong temporal PSF dependences. The results discussed here are for two distinct observation dates. After inspection of a coarse grid of PSFs for different observation dates, we selected TINY TIM PSFs for 1998 February 1 (as an example of a fairly blurred PSF) and "after 2002 September 29" (fully installed cryocooler phase, with only minor PSF blurring). 

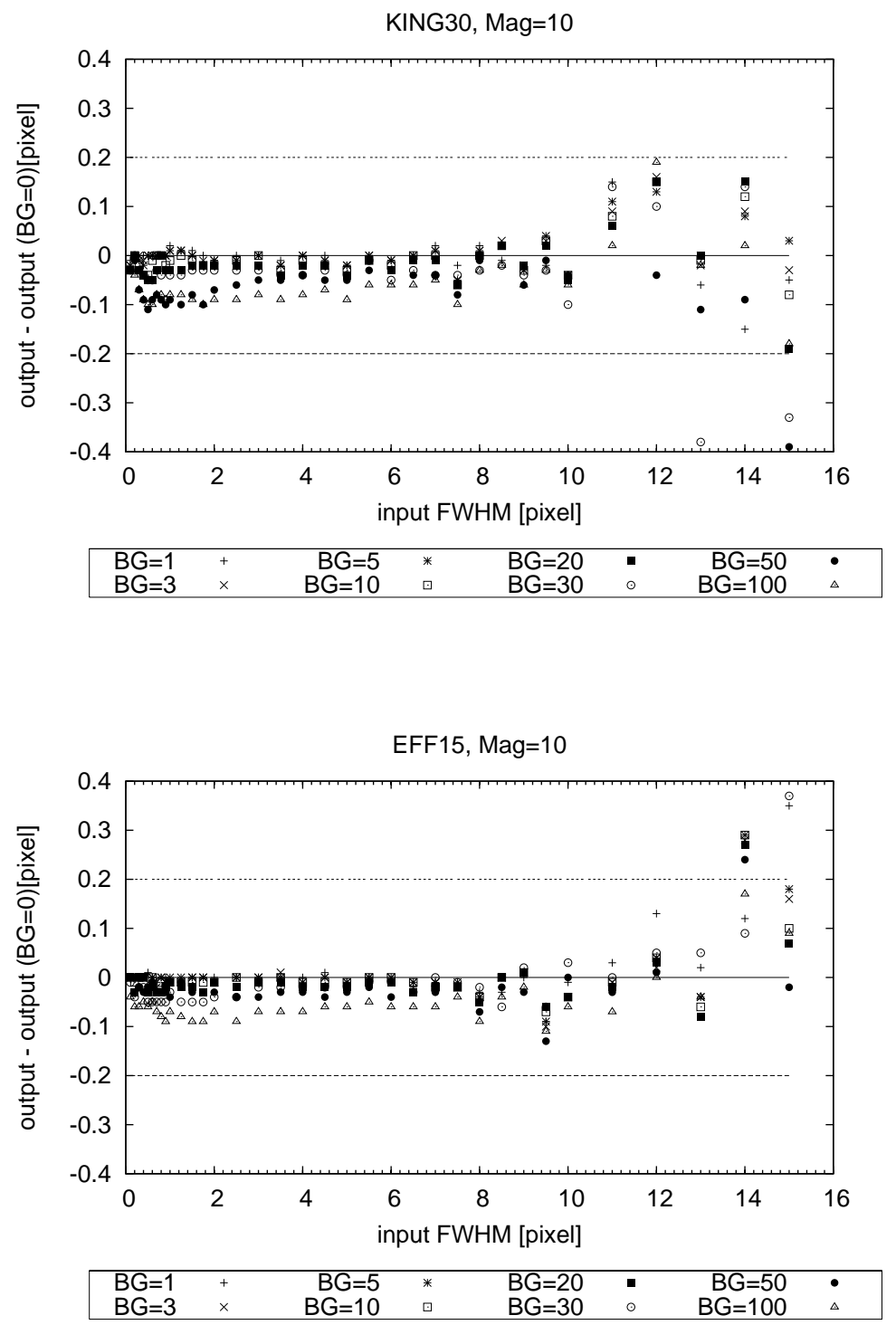

Figure 6.13: Conversion relations for a $V=10$ mag cluster, taking sky noise into account. As reference, the data for a standard cluster are taken. The straight lines are at \pm 0.2 pixel.

In both cases, a strong filter dependence is apparent, as can be seen in Fig. 6.19. For this reason, we give size conversions for all filters analysed (NICMOS equivalents to $J, H$ and $K$ ) and both epochs of observations in Tables $6.13-6.16$.

\subsubsection{Further sources of uncertainties}

Before assessing further possible sources of uncertainties we remind the reader that our study does NOT aim at working at the spatial resolution limit of HST (marginally resolved sources were already studied in Carlson \& Holtzman 

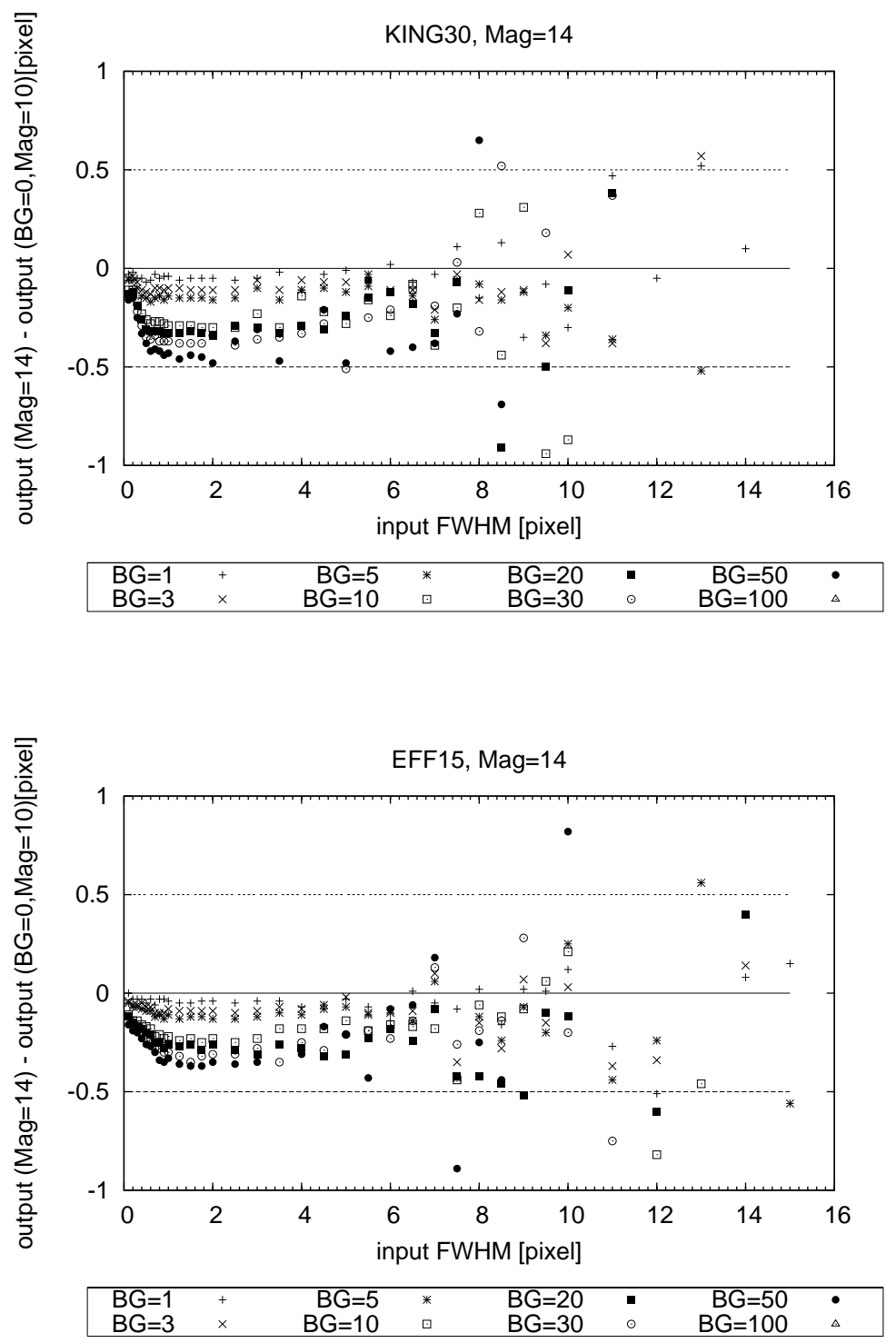

Figure 6.14: Conversion relations for a $V=14$ mag cluster, taking sky noise into account. As reference, the data for a standard cluster are taken. The straight lines are at \pm 0.5 pixel.

2001), but advice the users to apply our recipes only for clusters with an intrinsic FWHM greater than 0.5 pixel. Hence many of the tiny details and uncertainties inherent to PSF modelling (both theoretically as TINY TIM and using observed PSFs) smear out, becoming irrelevant for our studies.

Drizzling and jitter might blur images slightly by broadening the PSF. However, as Carlson \& Holtzman 2001 stated already (for an even more difficult situation, due to their smaller clusters, compared to ours) both effects have minor impact. The exact amount depends on the brightness of the source, the quality of the image reduction software to perform subpixel alignment, the number of exposures stacked, the length of these exposures etc.. 

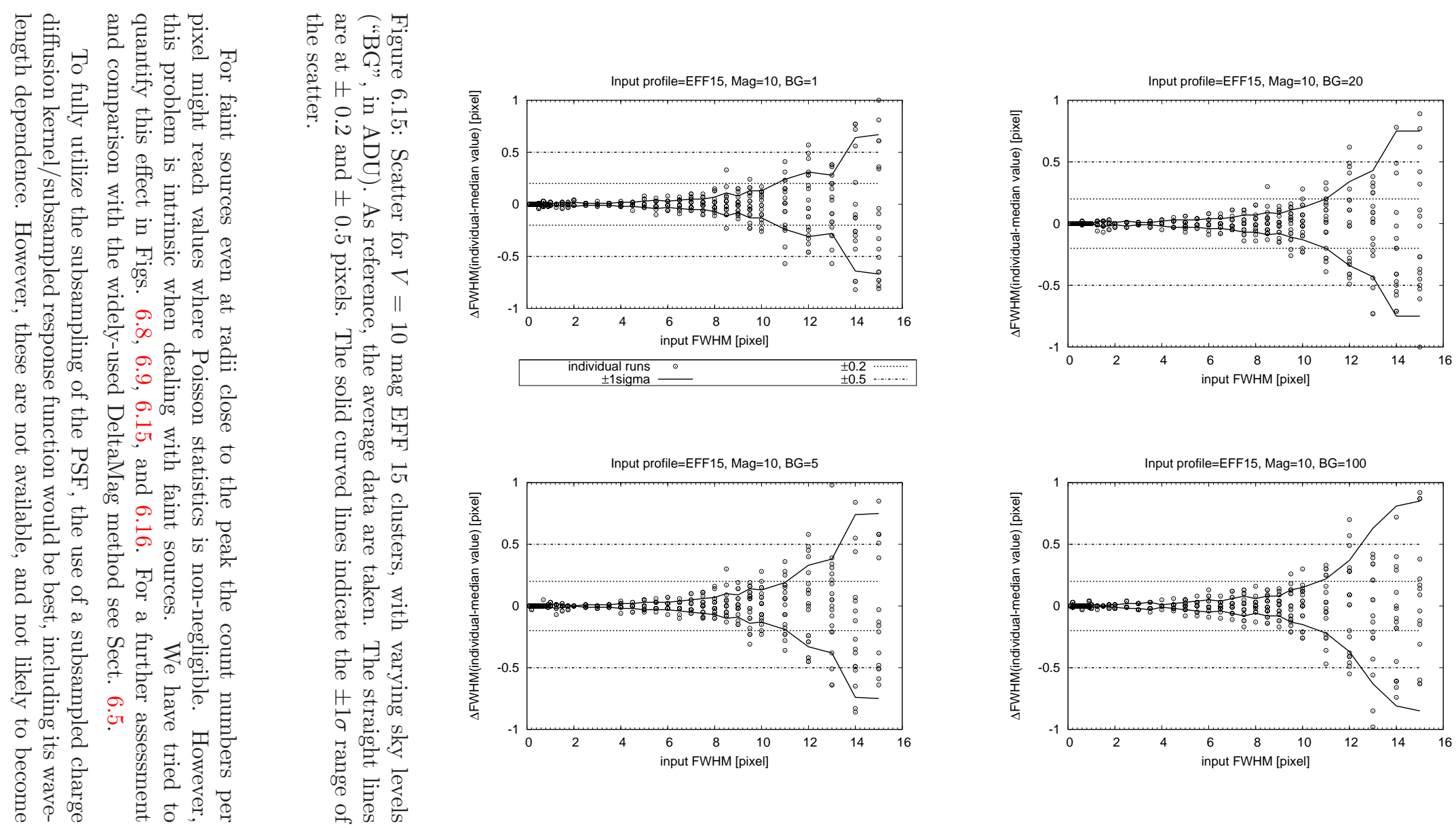

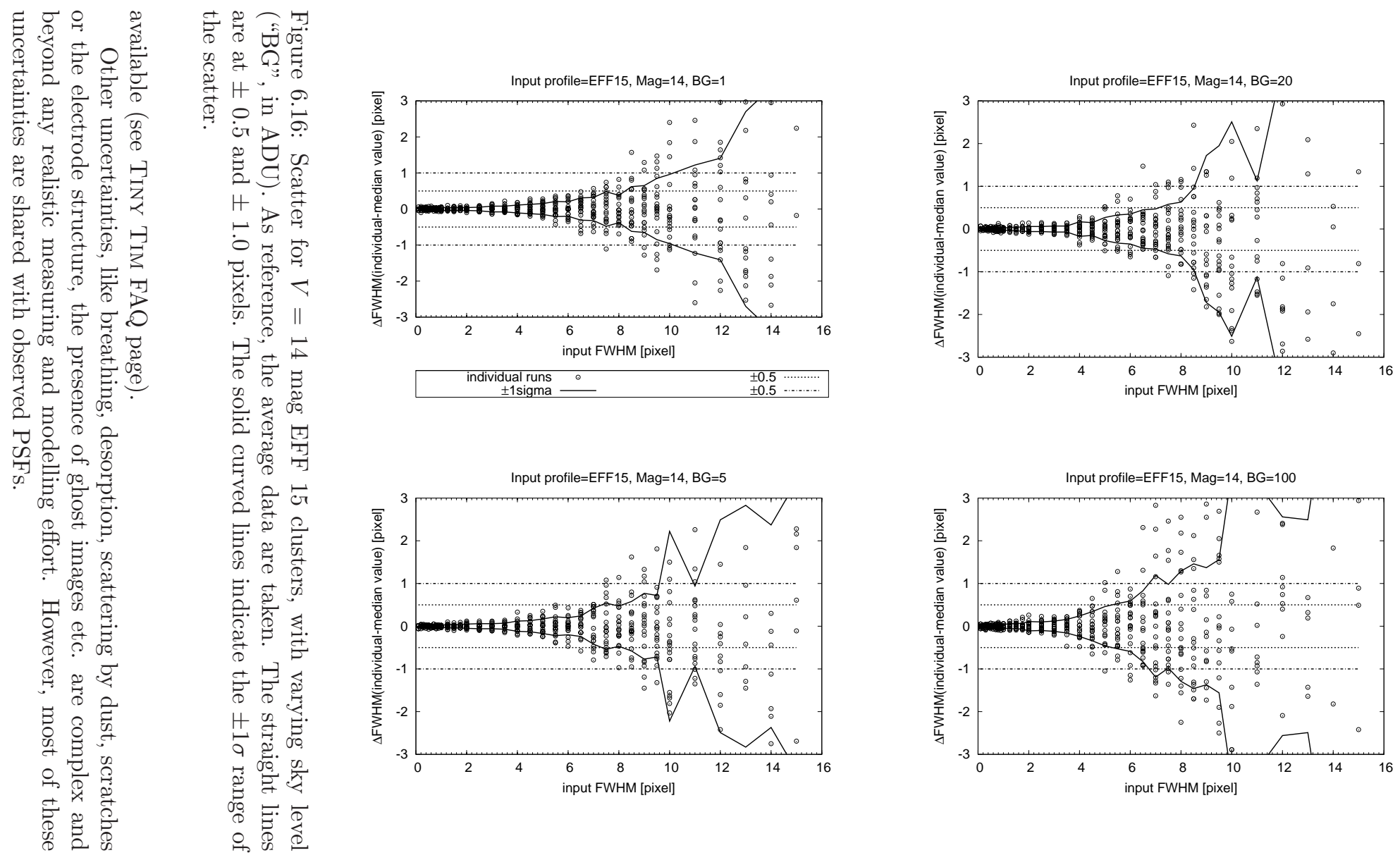

$\vec{\infty}$
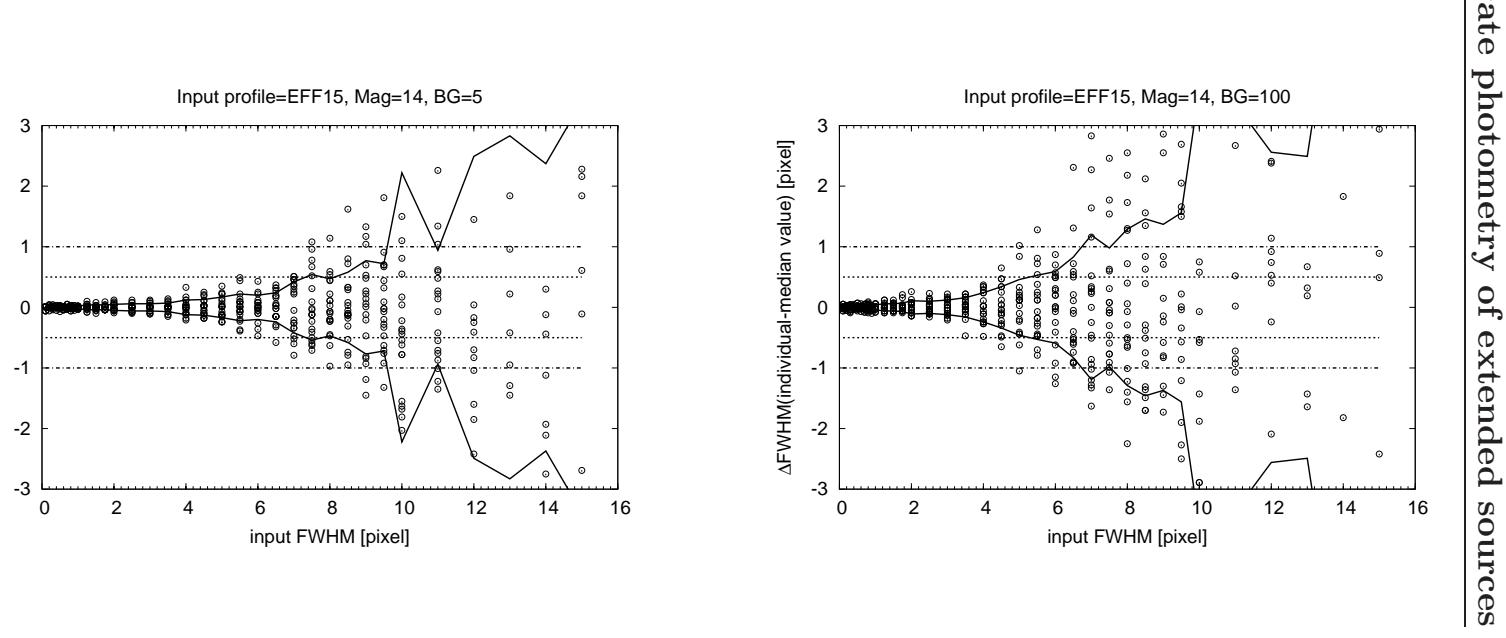

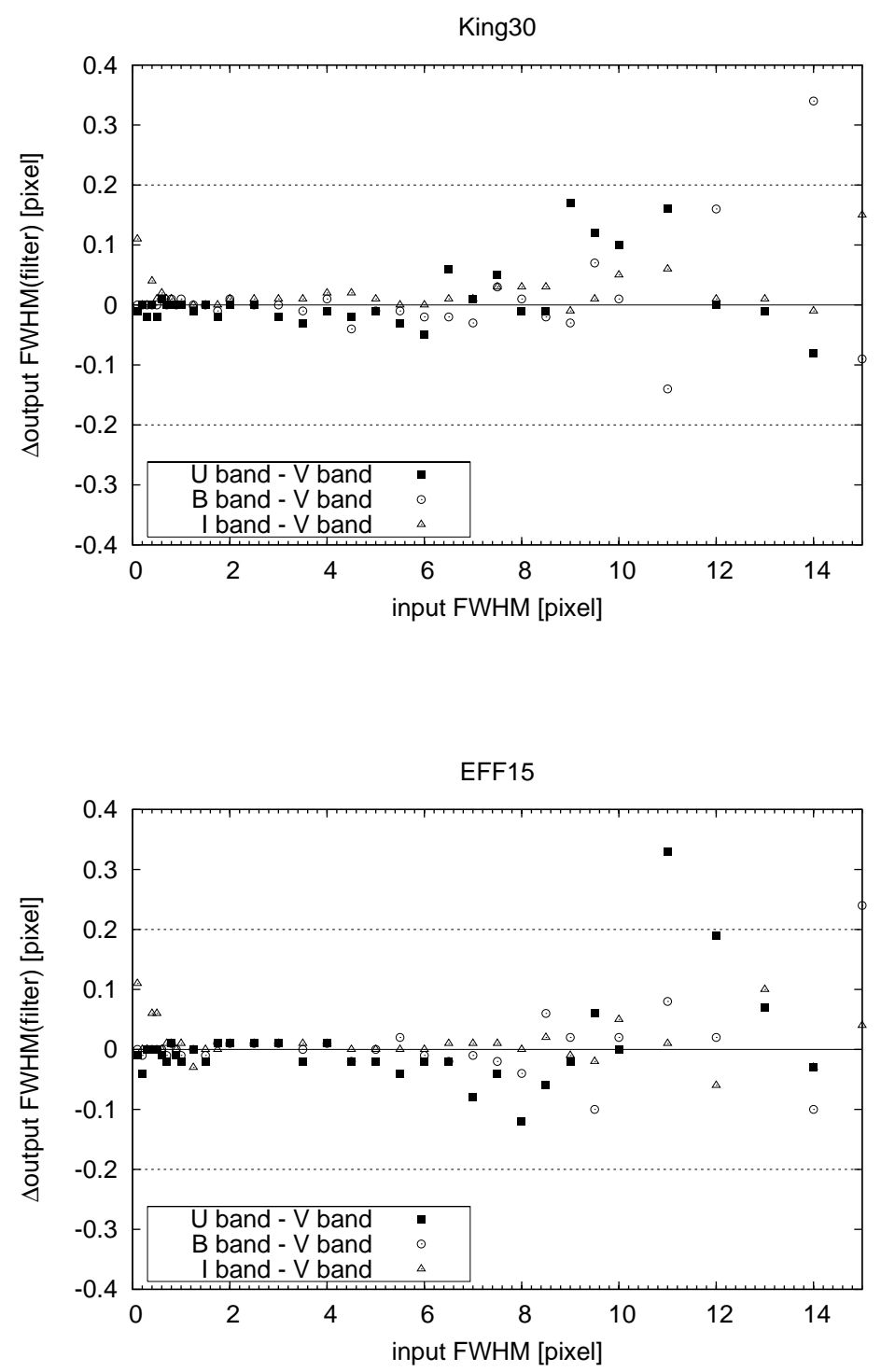

Figure 6.17: Conversion relations for a standard cluster, using the appropriate PSFs for the fitting. The horizontal lines indicate \pm 0.2 pixel. 

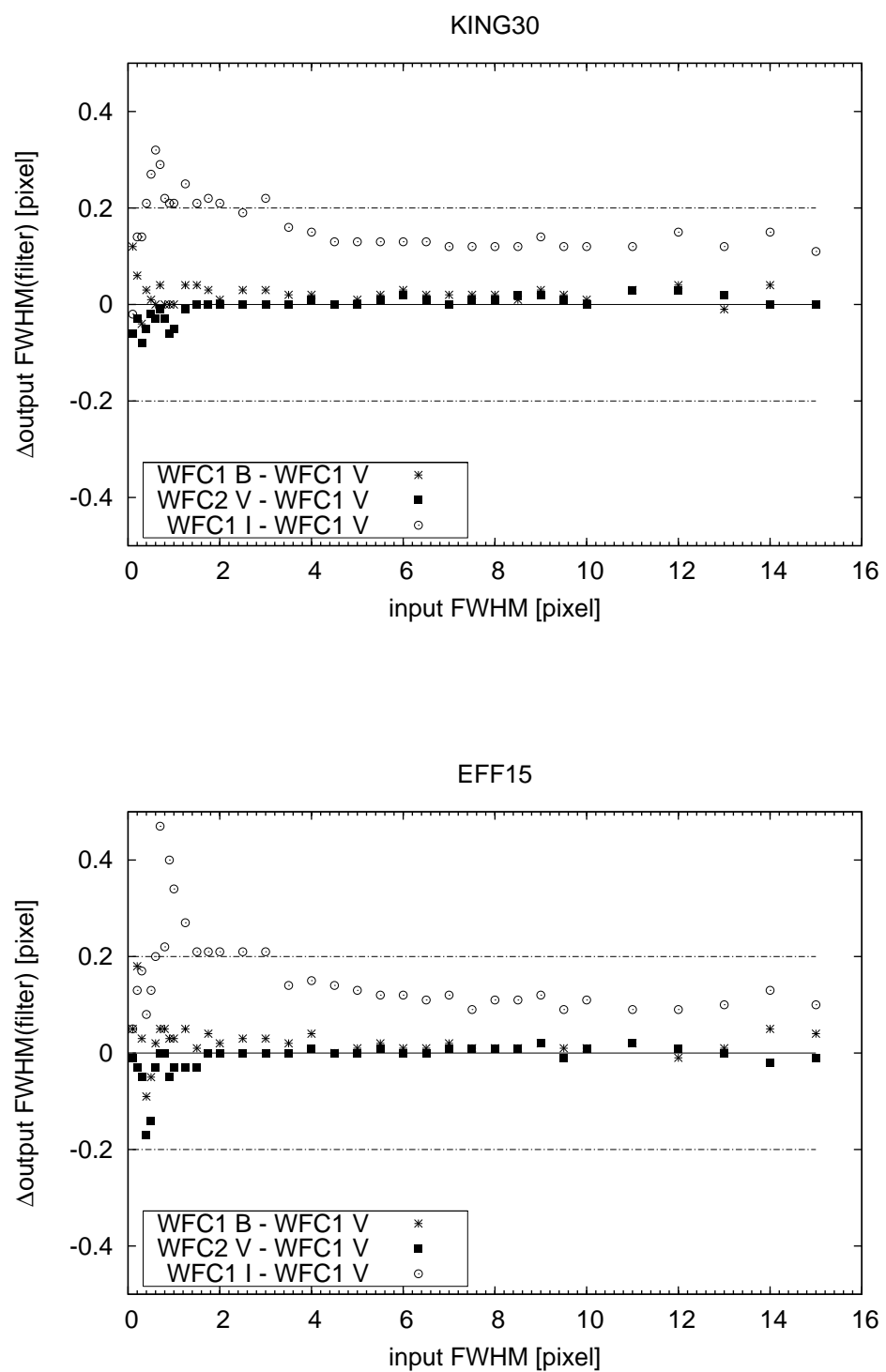

Figure 6.18: Conversion relations for a standard cluster, using different filters for the ACS/WFC (chip 1). 

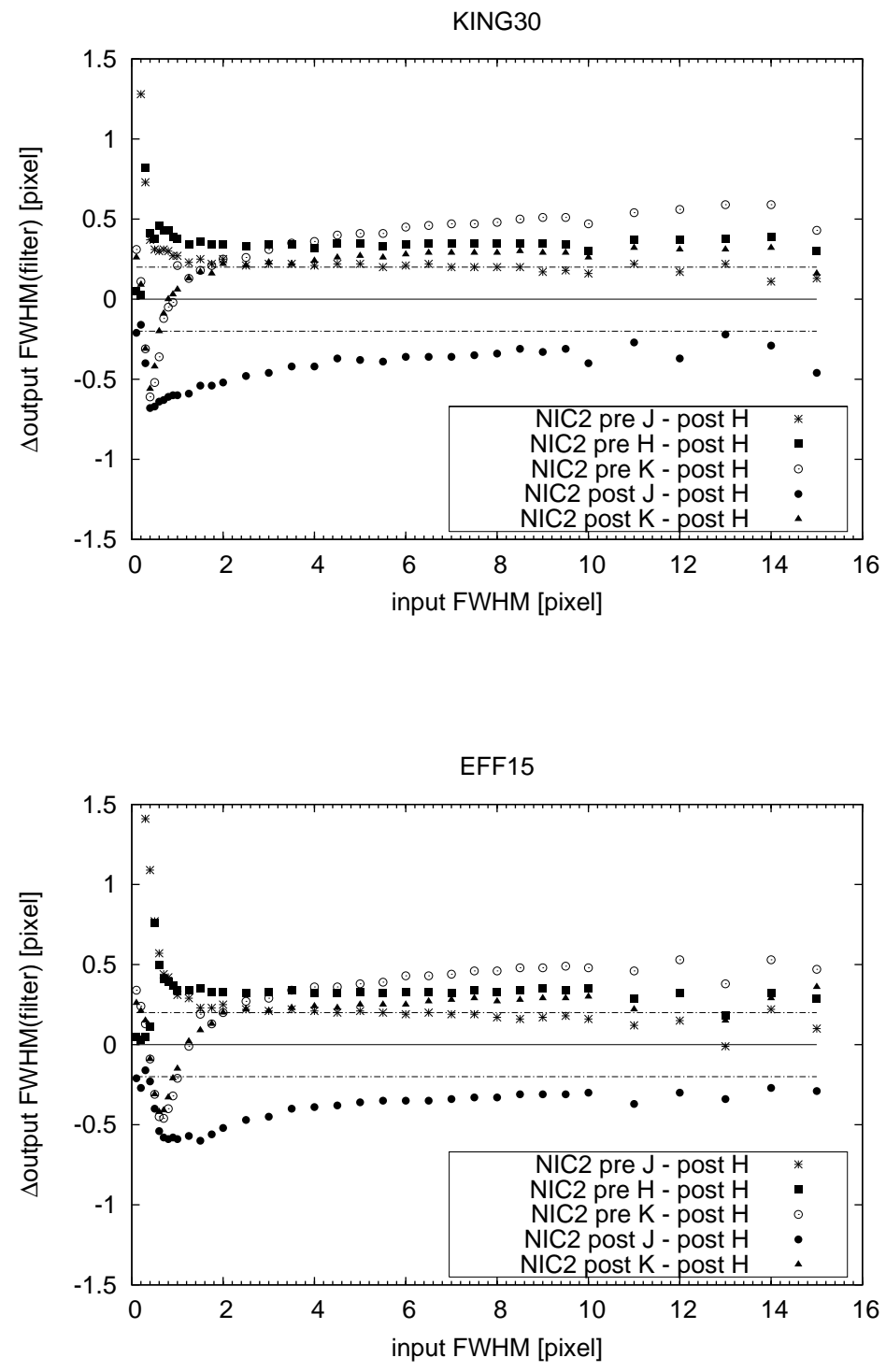

Figure 6.19: Conversion relations for a standard cluster (Upper panel: King 30 profile; Lower panel: EFF 15 profile), using different filters for NICMOS camera 2 . 


\subsection{Determining accurate photometry: Aper- ture corrections}

\subsubsection{Input parameters}

We generate artificial clusters of different light profiles and sizes, $R_{\mathrm{cl}}$, using the BAOLAB package and convolve them with the PSFs and diffusion kernels appropriate for the different cameras. We then determine ACs as a function of the FWHM of the source and the size of the aperture, $R_{\mathrm{ap}}$.

The aperture correction $\left(\mathrm{AC}_{\lambda}\right)$ is defined as:

$$
\begin{aligned}
\mathrm{AC}_{\lambda} & =-2.5 \log \left(F_{\lambda}\left(R_{\mathrm{ref}}, R_{\mathrm{cl}}\right) / F_{\lambda}\left(R_{\mathrm{ap}}, R_{\mathrm{cl}}\right)\right) \\
& =\operatorname{mag}_{\lambda}\left(R_{\mathrm{ref}}, R_{\mathrm{cl}}\right)-\operatorname{mag}_{\lambda}\left(R_{\mathrm{ap}}, R_{\mathrm{cl}}\right)
\end{aligned}
$$

where $F_{\lambda}(R)$ is the flux within an aperture with radius $R$, and $\operatorname{mag}_{\lambda}(R)$ the corresponding magnitude, both for a given wavelength (filter) $\lambda$. The "ref(erence)" radius, $R_{\text {ref }}$, is either infinity or another radius taken for reference, e.g., 0.5 arcsec (as recommended, e.g., by Holtzman et al. 1995). However, we will show that correcting to 0.5 arcsec, while appropriate for point sources, is insufficient for extended objects.

We consider three cluster light profiles in the remainder of this study:

- King 5: King profiles with $c=5$. This corresponds to the average concentration index observed for Galactic open clusters (e.g., Binney \& Tremaine 1998); we emphasise, however, that because of the peculiar cluster profile and the large extent of the HST PSFs, the size and AC relations for King 5 profiles are more uncertain than for the other profiles;

- King 30: King profiles with $c=30$, corresponding to the average concentration index observed for Galactic globular clusters (e.g., Binney \& Tremaine 1998);

- EFF 15: Elson, Fall \& Freeman models with a power-law index of 1.5, matching the average observed profile of young populous LMC clusters (Elson et al. 1987).

Clusters with FWHMs between 0.1 and 15 pixels were considered, for each chip. For the clusters with small FWHMs, PSF photometry might be a more accurate solution. For this purpose the HSTPнот package of Dolphin (2000) is available. However, since this package only works with very specific data formats, we cannot present a direct comparison of both methods here. Clusters larger than 15 pixels FWHM are very unlikely to occur, in particular since they must be very bright to have sufficiently high $\mathrm{S} / \mathrm{N}$ ratios out to such large radii. For such clusters, additional effects become important, including background contamination and crowding.

The analysis was done for the WFPC2 PC and WF3 chips, for the ACS/WFC1 (WFC2 is equivalent) and for the NICMOS/NIC2 (pre and post-cryocooler) chips.

After generating and convolving the clusters with the appropriate PSF and diffusion kernel (where relevant), aperture photometry of the noiseless objects was done using different apertures. The ACs were determined with respect to 
the reference aperture. If not otherwise specified $R_{\text {ref }}=50$ pixels is used. No model exhibits strong changes in the light profile for such large radii, hence $R_{\text {ref }}=50$ pixels is sufficiently close to $R_{\text {ref }}=\infty$ for our purposes.

\subsubsection{The relation between aperture correction and input FWHM}

First, we determine the relation between $\mathrm{AC}$ and input FWHM of the object.

The result is again fitted with a fifth-order polynomial function,

$$
\mathrm{AC}(x)=a+b * x+c * x^{2}+d * x^{3}+e * x^{4}+f * x^{5},
$$

where $x$ is the input FWHM of the object (in pixels), and $a$ through $f$ are the fitting coefficients.

An example is shown in Fig. 6.20. The bottom panel shows that the differences between the data and the fits of the form of Eq. (6.7) are smaller than 0.0025 mag. Hence, the fits are very accurate.

In Holtzman et al. (1995), the amount of missed light outside a 0.5 arcsec aperture is estimated to be $-0.1 \mathrm{mag}$ for point sources. Fig. 6.21 shows that the correction to a radius of 0.5 arcsec deviates by much more than -0.1 mag from the correction to an infinite aperture for extended objects. This confirms, again, the importance of correcting for the size of an object, and of including all of its flux.

All of the fit results are tabulated in Tables 6.17-6.43.

\subsubsection{The relation between aperture correction and mea- sured FWHM}

By combining the results from Sections 6.2 and 6.3.2, we can now determine the more immediately applicable $\mathrm{AC}$ values as a function of the measured FWHM.

An example of the fit results is shown in Fig. 6.22. All of the fit results are tabulated in Tables 6.44-6.70.

The polynomial fits to the data using the measured FWHM are less satisfactory than the fits using the intrinsic sizes. The deviations are, depending on the cluster profile and the PSF used, on the order of $\pm 0.01-0.1 \mathrm{mag}$, distributed fairly homogeneously over this range; see Fig. 6.22 for two extreme examples.

\subsubsection{Sky oversubtraction}

The most often used and most practical way to subtract the sky background from the cluster light is by defining a sky aperture around the cluster and subtracting the sky level. However, in most cases the sky annuli have to be chosen fairly close to the cluster to avoid confusion with nearby clusters or stars (i.e., crowding), gradients and strong variations in the sky background, among others. Because of the possibly large extent of the combination of the cluster and HST PSF, this "sky" subtraction likely also subtracts cluster light, in general. To obtain the actual cluster magnitude, this oversubtraction must be corrected for. We found as correction term (in magnitudes):

$$
\Delta \operatorname{mag}_{1}=2.5 \log (1-A \times F) \quad,
$$



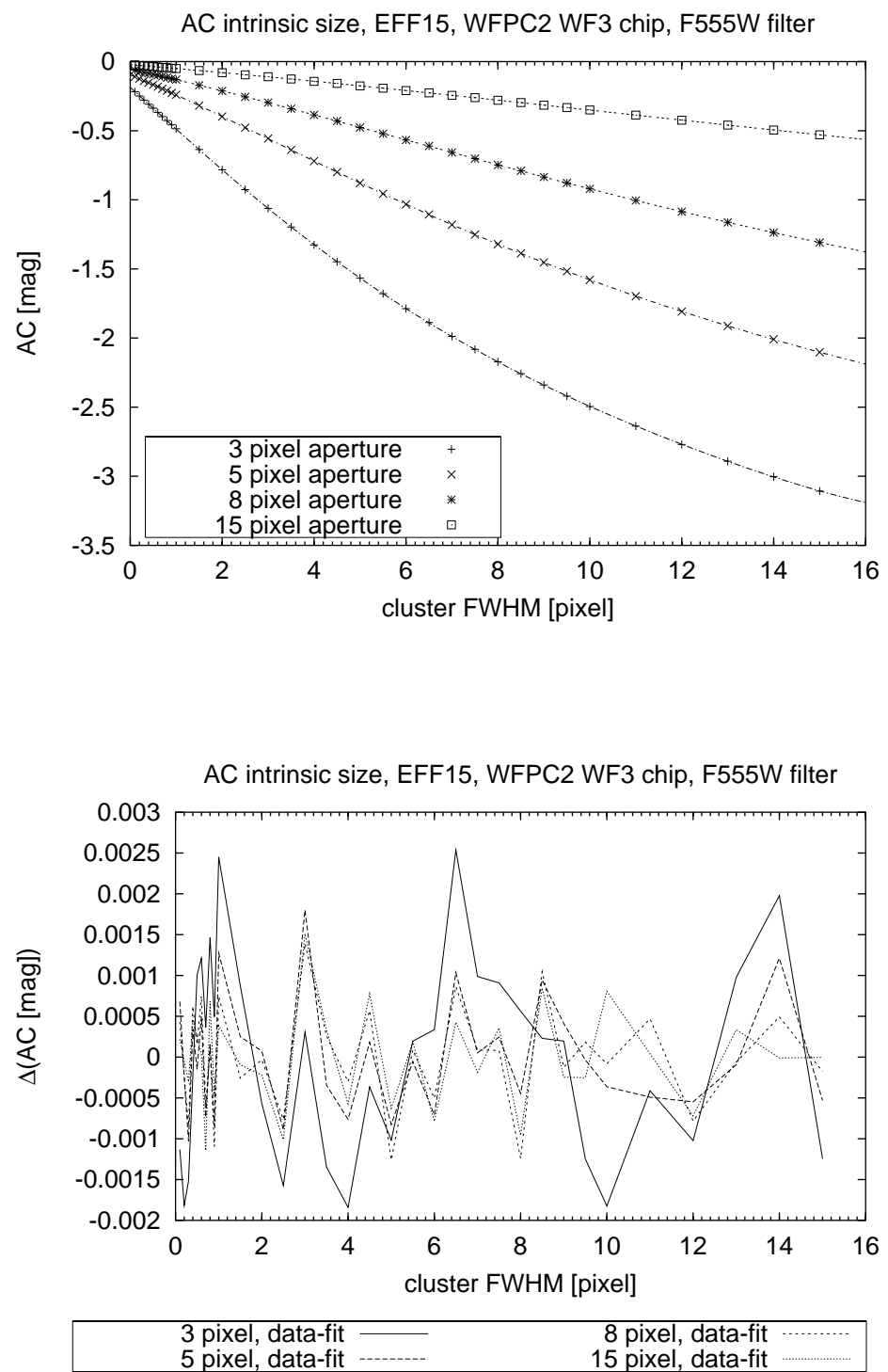

Figure 6.20: Upper panel: Theoretical AC values (to infinite radius) for EFF 15 profiles with different FWHMs, and best fit results. The aperture sizes used are given. Lower panel: Deviations of data from fits.

with area ratio

$$
A=\frac{r_{1}^{2}}{r_{3}^{2}-r_{2}^{2}}
$$

and flux ratio

$$
F=\frac{10^{0.4 \mathrm{AC}\left(r_{3}, s\right)}-10^{0.4 \mathrm{AC}\left(r_{2}, s\right)}}{10^{0.4 \mathrm{AC}\left(r_{1}, s\right)}}
$$

where $r_{1}, r_{2}$ and $r_{3}$ are the sizes of the source annulus, and inner and outer sky annuli (in pixels), $s$ is the measured cluster FWHM (in pixels), and $\mathrm{AC}\left(r_{i}, s\right)$ 


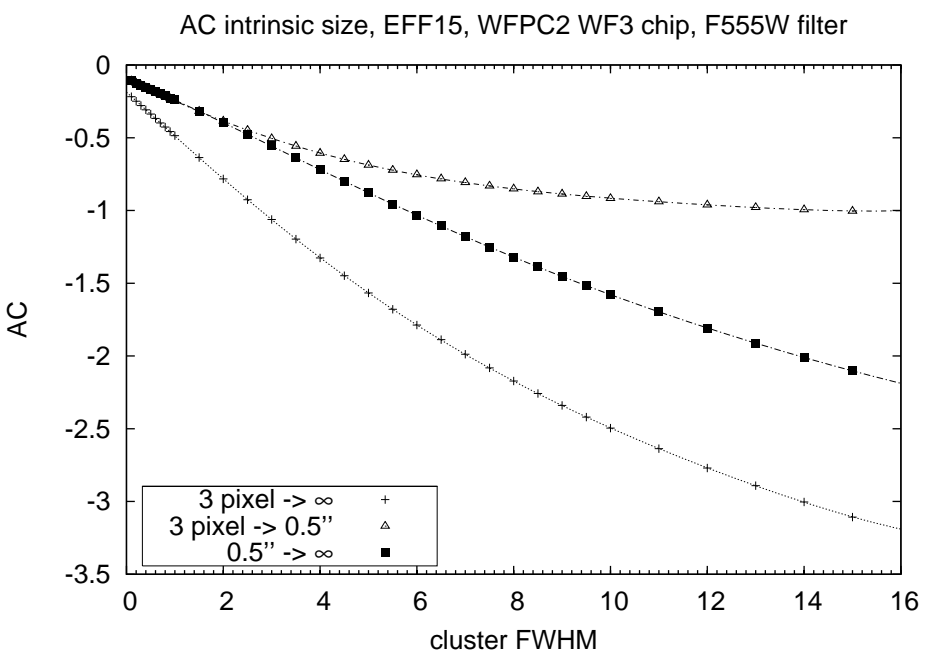

Figure 6.21: Theoretical AC values for EFF 15 profiles with different FWHMs, and best fit results.

The aperture size used is 3 pixels: $\Delta$ corrected to a 0.5 "aperture; + corrected

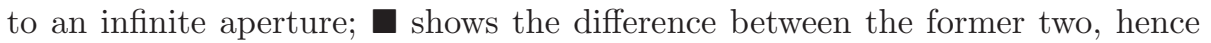
the correction $0.5^{\prime \prime} \rightarrow \infty$.

are the aperture corrections (given in Tables 6.17-6.43) for a given cluster size and given annuli. An example is given in Fig. 6.23. Using either $\mathrm{AC}_{\text {meas }}$ and $s_{\text {meas }}$ or $\mathrm{AC}_{\text {intr }}$ and $s_{\text {intr }}$ yields the same results.

\subsubsection{Filter dependence}

The same analysis as in Section 6.3.2 was done for all WFPC2 filters. Suchkov \& Casertano (1997) found that for apertures of 3 or more pixels, the largest filter dependence (compared to F555W) of the ACs was 0.06/0.03 mag (in the F814W band, for the PC/WF3 chips, respectively). From 5 pixels onward and for the F439W band (the only other band apart from F555W and F814W considered in Suchkov \& Casertano 1997), the differences are on the order of $0.01 \mathrm{mag}$. For the extreme case of a 3-pixel aperture, the differences between the F336W, F439W and F814W bands with respect to the F555W band are shown in Fig. 6.24. We clearly confirm the results of Suchkov \& Casertano (1997). Only for the F814W band of the PC chip we get 0.08 mag, i.e., 0.02 mag larger than Suchkov \& Casertano (1997), but most likely within the combined uncertainties of both studies.

\subsubsection{Subpixel shifts of clusters and the impact on the aperture corrections}

Since observed clusters do not exhibit a smooth analytic profile but are modified by the pixel structure of the chip, subpixel shifts of the clusters and the accompanying redistribution of counts can change the photometry of the clusters and the aperture corrections. The changes are expected to be strongest for small 

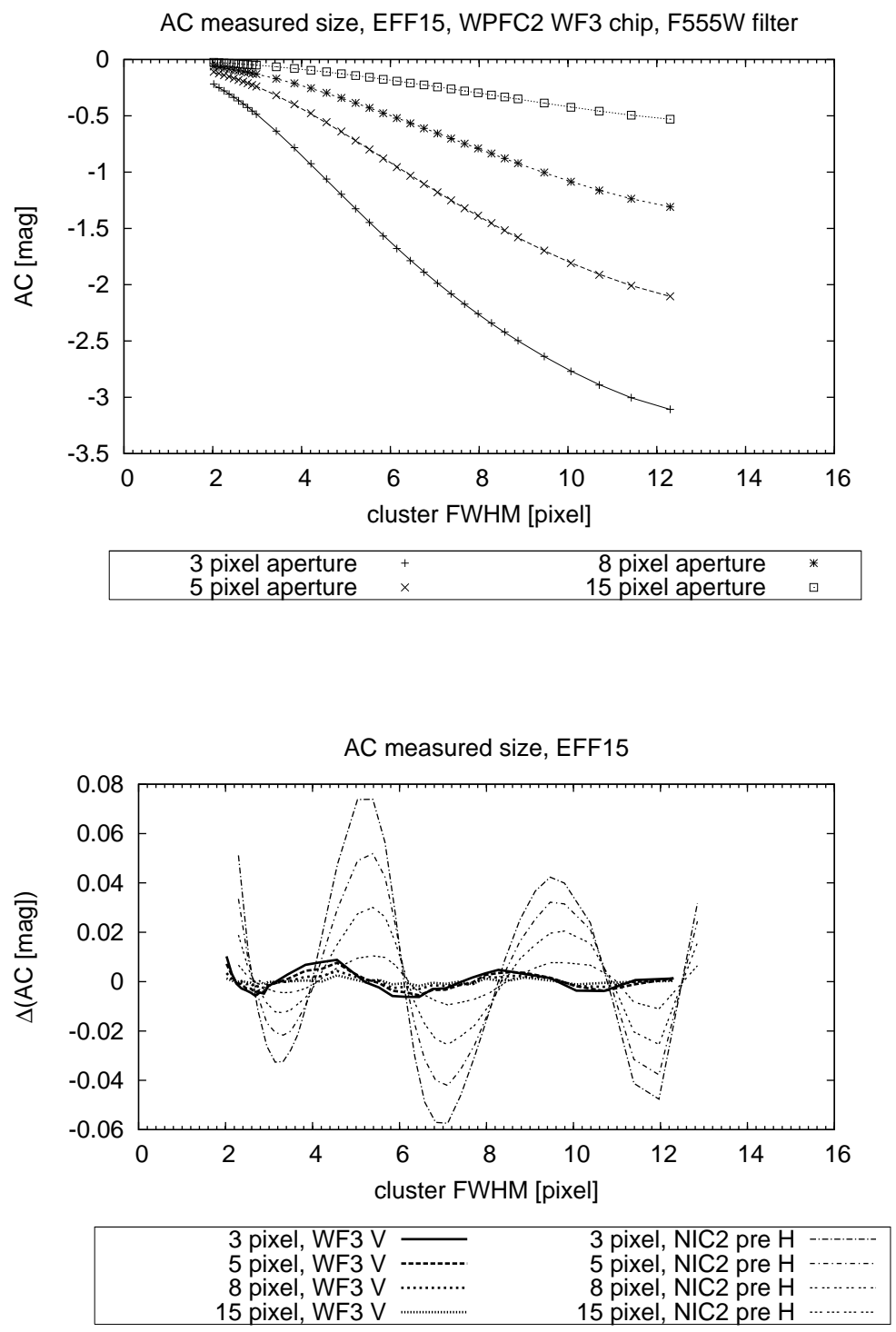

Figure 6.22: Upper panel: Theoretical AC values (to infinite radius) for EFF 15 profiles with different measured FWHMs, and best fit results. The aperture sizes used are given. Lower panel: Deviations of data from fits, covering the whole range of fit accuracies.

apertures. In Fig. 6.25 we show the absolute differences of ACs for differently centered clusters (and the photometry annuli centered at the cluster). In Fig. 6.26 we show the relative differences of ACs for differently centered clusters (and the photometry annuli centered at the cluster). The figures show the expected behaviour of smaller apertures suffering from larger deviations. For 3 pixel annuli the differences caused by centering changes can be up to $0.25 \mathrm{mag}$, but for larger annuli even the maximum deviations are below $0.1 \mathrm{mag}$ (corresponding to deviations of less than 20 per cent in almost all cases). 


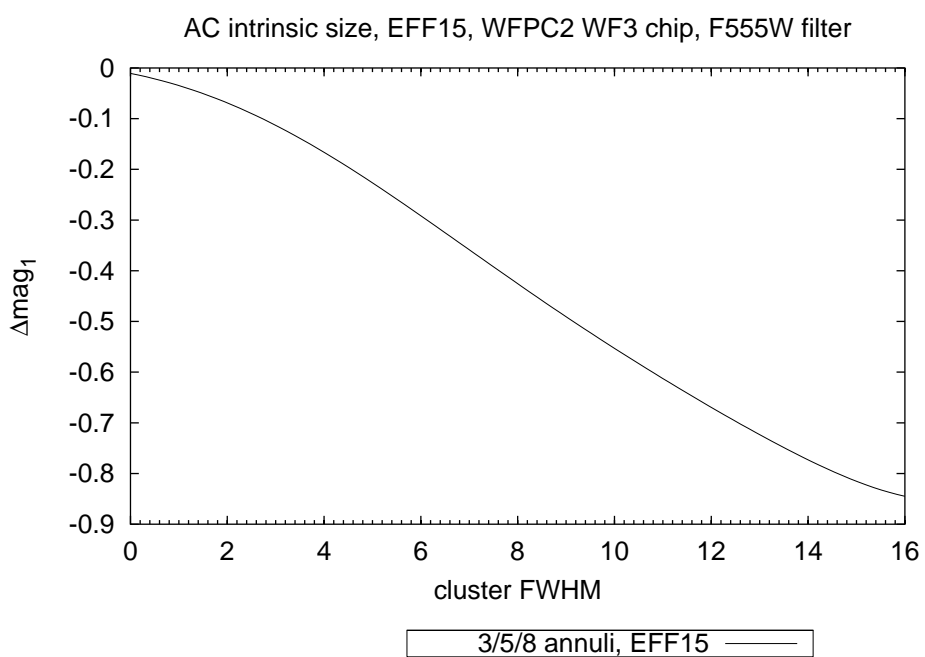

Figure 6.23: Correction for sky oversubtraction as a function of input FWHM, following Eq. (6.8), for a 3-pixel source annulus, 5/8-pixel inner/outer sky annulus, and an EFF 15 profile.

The photometric centering is much less of an issue for our method, thanks to the fairly large apertures. The deviations are always below $0.04 \mathrm{mag}$, corresponding to less than 13 per cent in all cases. This is shown in Figs. 6.27 and 6.28 .

\subsection{Cookbook for size-dependent aperture cor- rections}

This subsection describes the most efficient use of the tables presented in this paper to apply to observations. We will use one object as an example.

- Fit a Gaussian profile to your source, using the appropriate parameters:

- Use a fitting radius of 5 pixels (as shown in Section 6.2.4, the fitting radius has a significant impact on the size determination).

- Use the co-added images also used for the photometry. We suggest the use of images roughly in the wavelength range between the $B$ and $I$ band, unless some of these filters have significantly lower $\mathrm{S} / \mathrm{N}$ ratios than other available filters.

- Determine the flux-weighted mean of the sizes. This assumes a wavelengthindependent size, and hence no mass segregation or similar effects. If there is good reason to expect such effects one should treat the photometry of each filter independently. 

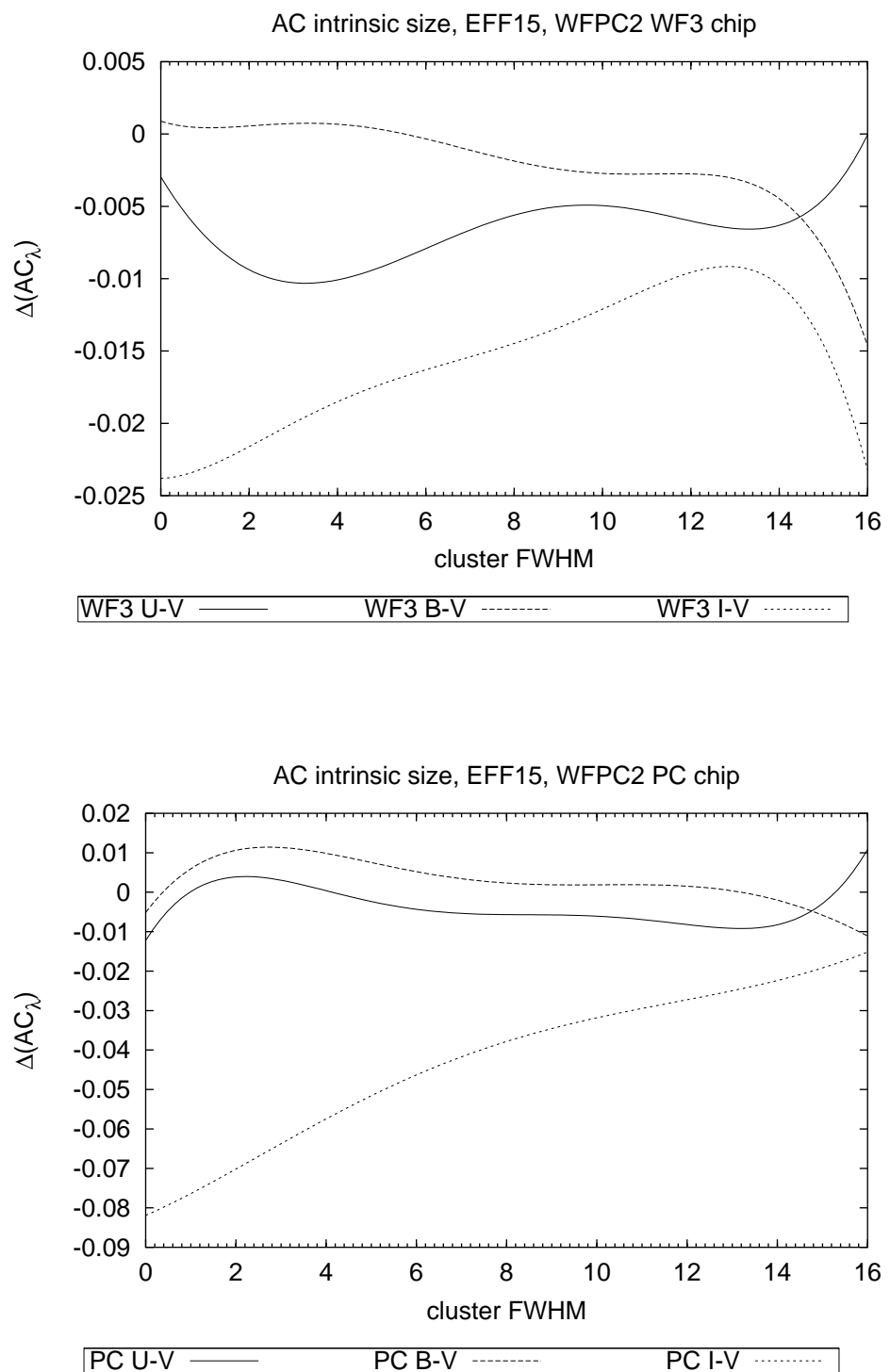

Figure 6.24: Filter dependence of the $\mathrm{AC}$ for a 3-pixel aperture, in $U B V I$ for the WF3 (upper panel) and the PC (lower panel) chip of the WFPC2, and assuming an EFF 15 profile.

- If the size that is determined is smaller than one of the relevant PSF sizes (see Table 6.3) and larger than an apparently reasonable lower size cut-off (perhaps on the order of $0.5-1.0$ pixels; sources with even smaller radii will most likely be spurious detections), set the size to the PSF size, which can be found in Table 6.3 (these sources are most likely point sources).

- Choose the most relevant cluster profile; see Section 6.3.1 for help.

- Perform (circular) aperture photometry by choosing appropriate source and sky annuli. 

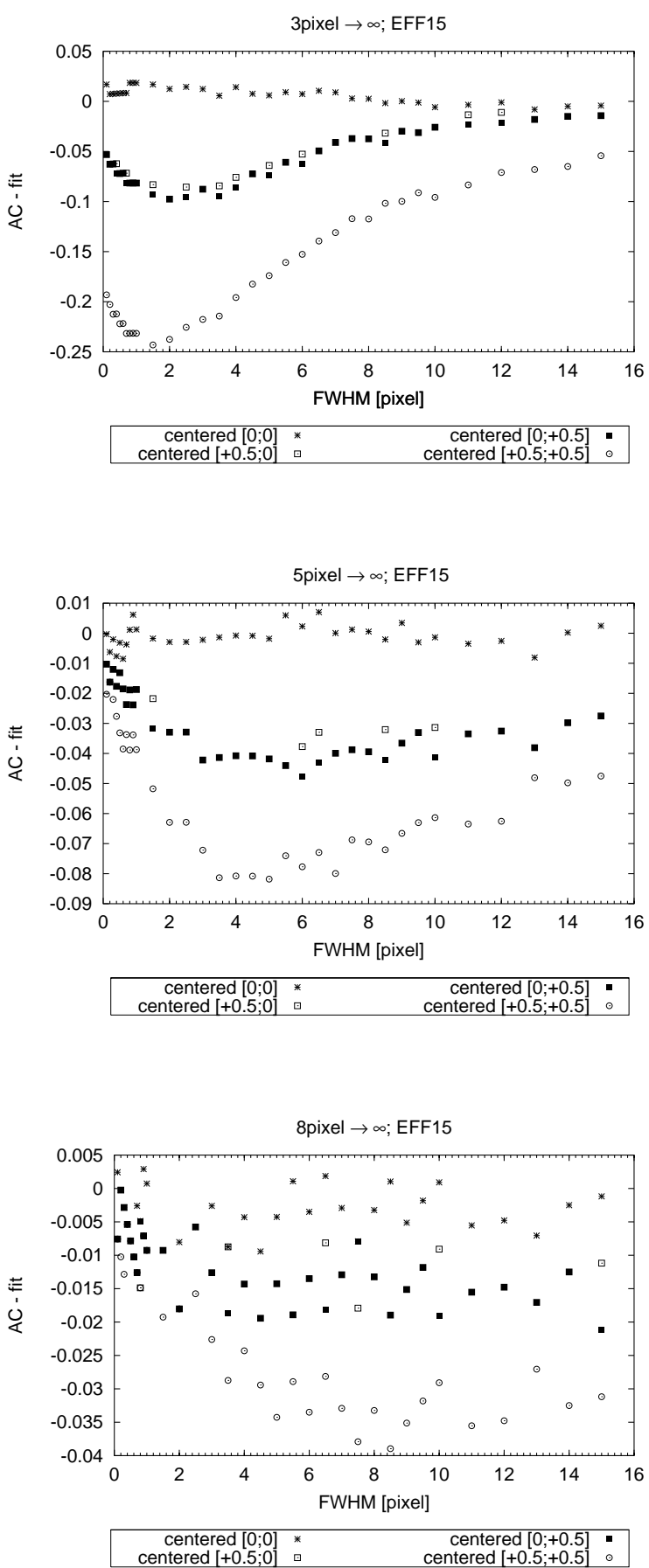

Figure 6.25: ACs for clusters with subpixel shifts relative to the $[0 ; 0]$ point, assuming a standard cluster with an EFF 15 profile. Shown are the absolute deviations between differently centered clusters with respect to the fit for the cluster at $[0 ; 0]$. Top panel: AC 3 pixels $\rightarrow \infty$. Middle panel: AC 5 pixels $\rightarrow \infty$. Bottom panel: AC 8 pixels $\rightarrow \infty$. 

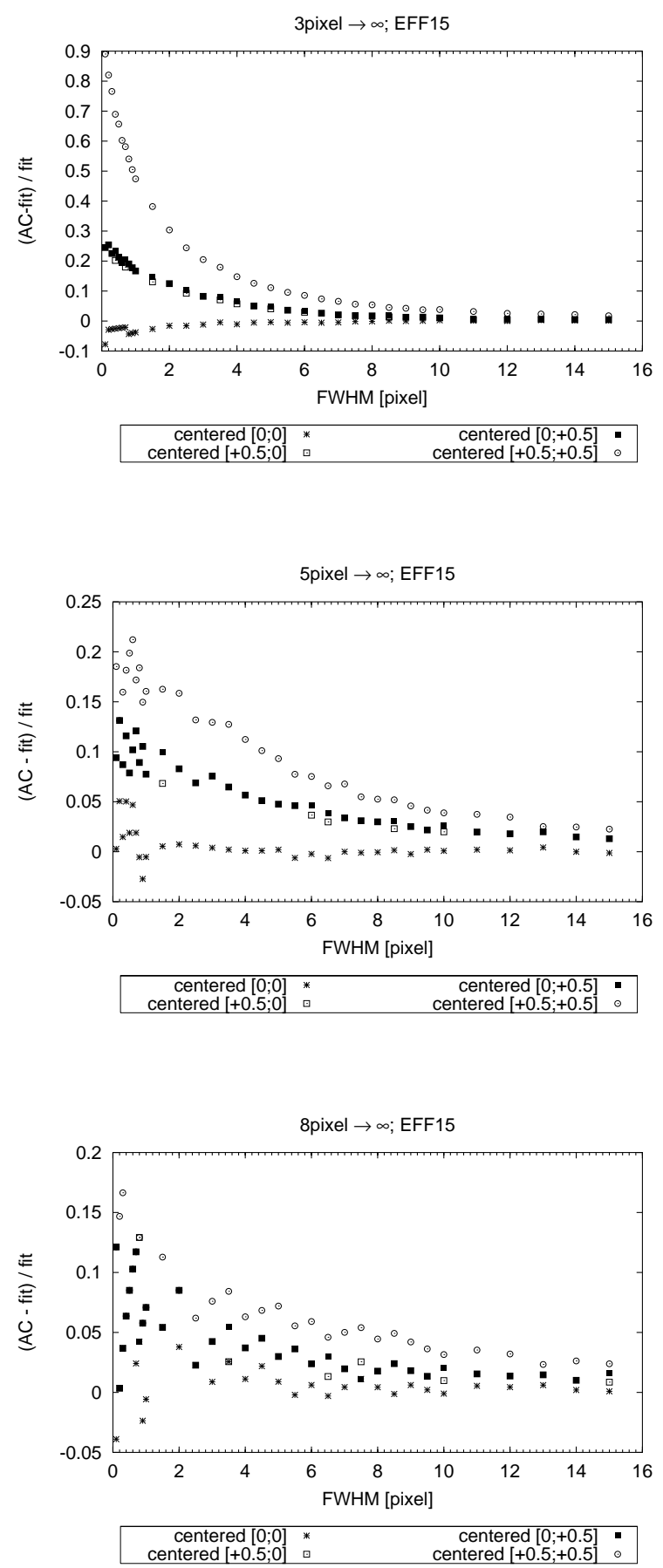

Figure 6.26: ACs for clusters with subpixel shifts relative to the $[0 ; 0]$ point, assuming a standard cluster with an EFF 15 profile. Shown are the relative deviations between differently centered clusters with respect to the fit for the cluster at [0;0]. Top panel: AC 3 pixels $\rightarrow \infty$. Middle panel: AC 5 pixels $\rightarrow \infty$. Bottom panel: AC 8 pixels $\rightarrow \infty$. 

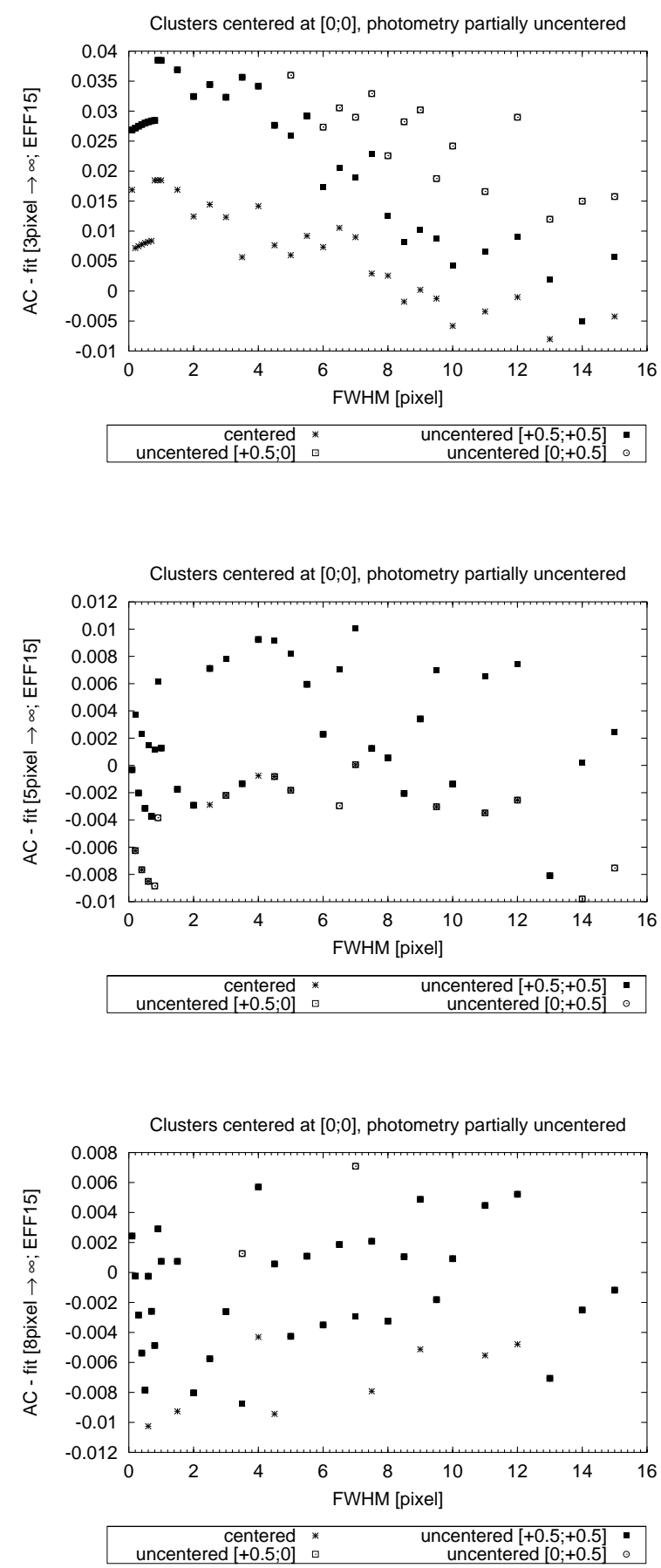

Figure 6.27: ACs for clusters with annuli shifts relative to the [0;0] point, assuming a standard cluster with an EFF 15 profile. Shown are the absolute deviations between differently centered annuli with respect to the fit for the cluster at [0;0]. Top panel: AC 3 pixels $\rightarrow \infty$. Middle panel: AC 5 pixels $\rightarrow \infty$. Bottom panel: AC 8 pixels $\rightarrow \infty$. 

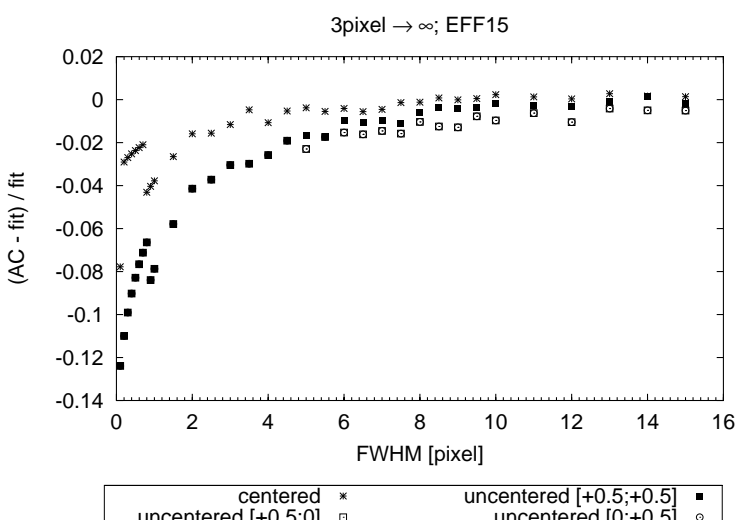
uncentered $[0 ;+0.5]$

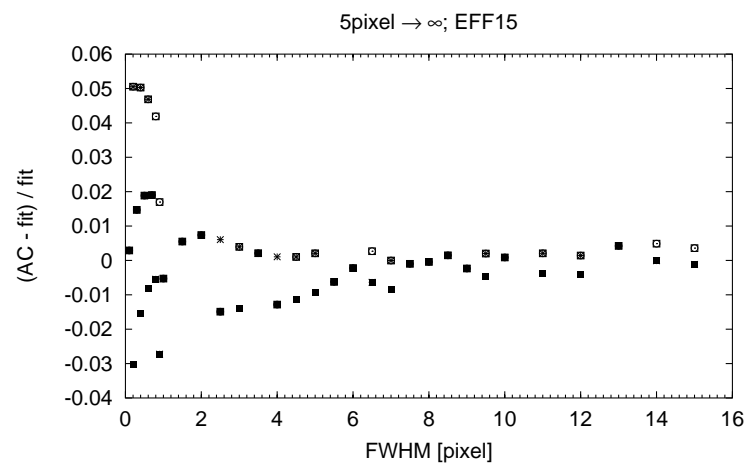

centered $*$ uncentered $[+0.5 ;+0.5] \quad$ -

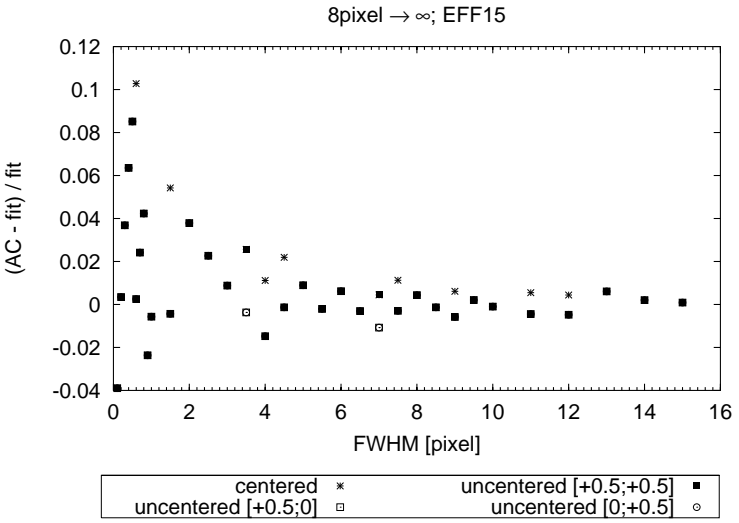

Figure 6.28: ACs for clusters with annuli shifts relative to the [0;0] point, assuming a standard cluster with an EFF 15 profile. Shown are the relative deviations between differently centered annuli with respect to the fit for the cluster at $[0 ; 0]$. Top panel: AC 3 pixels $\rightarrow \infty$. Middle panel: AC 5 pixels $\rightarrow \infty$. Bottom panel: AC 8 pixels $\rightarrow \infty$. 
- Calculate the aperture correction, using the annuli and measured size, and the polynomials from Tables $6.44-6.70$.

- Calculate the oversubtracted cluster light, using Eq. 6.8, the annuli and measured size.

- Add the measured cluster magnitude, the calculated aperture correction and oversubtraction correction to obtain the cluster magnitude. Check that both corrections are negative; otherwise set to zero.

\subsection{Comparison of our method with the widely used DeltaMag method}

\subsubsection{Size determination}

Since many authors prefer other methods to determine sizes and aperture corrections, we compare our results with results from the most widely used method in this section.

The most commonly used measure of size is the magnitude difference in two concentric apertures (hereafter referred to as the "DeltaMag method"). A commonly used definition involves apertures of radii 0.5 and 3 pixels. A first obvious difficulty of using 0.5 pixel radii apertures is the centering, the distribution of the photons onto the relevant pixels and the accurate measurement of this effect. In addition, a Gaussian profile is usually assumed.

In the following, we will use our BAOLAB cluster models to estimate the size determination accuracy for the DeltaMag method. We used our standard cluster settings and measured the magnitudes in apertures with radii of 0.5 and 3 pixels. For our three main models, the resulting magnitude differences as a function of input FWHM is shown in the top panel of Fig. 6.29. The impact of the centering is displayed in the middle and bottom panels of Fig. 6.29. Two types of centering have to be distinguished; (i) the centering (or exact positioning down to subpixel levels) of the cluster on the pixels of the CCD, and (ii) the photometric centering (the centering of the photometric annuli, or more generally the exact determination of the position of the cluster at subpixel levels). As shown in the middle and bottom panels of Fig. 6.29, the DeltaMag method is very sensitive to both kinds of centering problems.

We emphasize that the photometric centering is an integral part of BAOLAB; hence is not a major problem for our method. In addition, the impact of incorrect centering is much more severe for a 0.5-pixel aperture compared to our standard aperture of 3-pixel radius. See Section 6.3.6 for the impact of subpixel shifts on the ACs for our method.

Another source of uncertainties intrinsic to the DeltaMag method results from the photometric uncertainty for each annulus. Assuming a photometric accuracy in $\Delta \mathrm{mag} \simeq \pm 0.1 \mathrm{mag}$ (which might even be too small for the 0.5 pixel annulus, because of centering issues), we determine how far off the size determination gets. The results are shown in Fig. 6.30 for two cluster light profiles. As a comparison we plot the accuracy limits for our method, determined by the stochastic effects during cluster formation (generation). In both cases the maximum deviations are calculated, and shown in Fig. 6.30. This result 

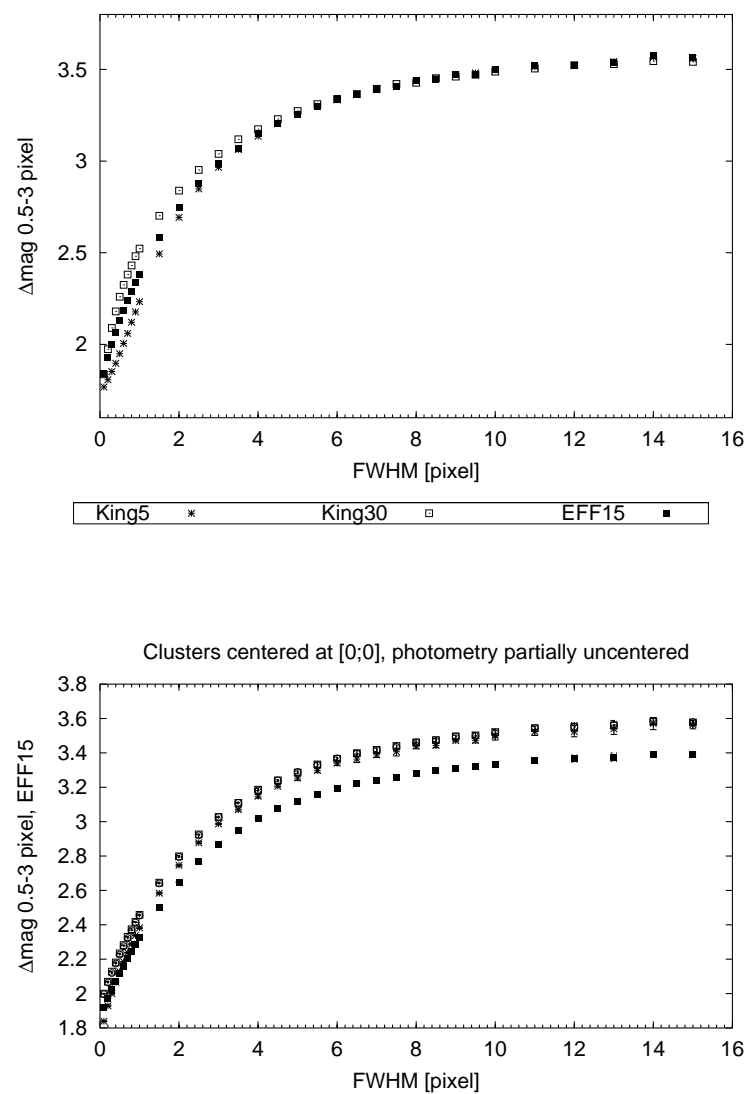

$\begin{array}{rr}\text { centered } & \text { uncentered }[+0.5 ;+0.5] \\ \text { uncentered }[+0.5 ; 0] & \text { uncentered }[0 ;+0.5]\end{array}$

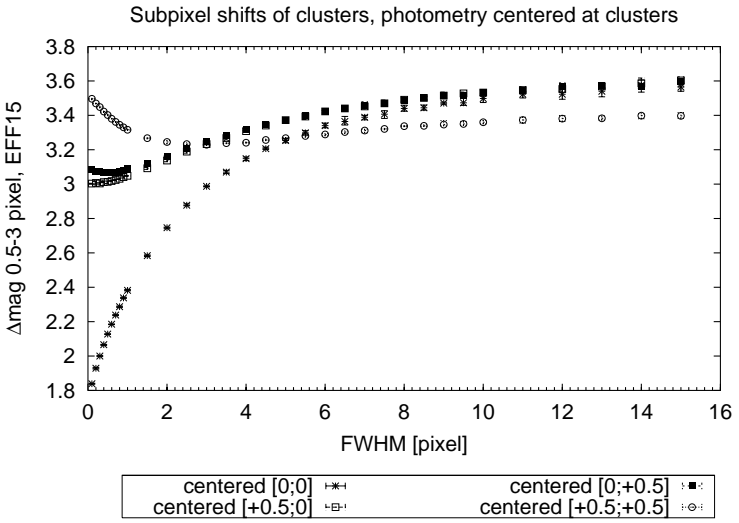

Figure 6.29: Top panel: $\Delta$ mag between 0.5 and 3 pixels, for 3 different profiles. Middle panel: Comparison of $\Delta \mathrm{mag}$ for off-centered aperture annuli for the EFF 15 profile. Bottom panel: Comparison of $\Delta$ mag for different centerings of clusters on a pixel, using an EFF 15 profile. 
further strengthens the confidence we have in our method. The improvement in accuracy from the DeltaMag to our method is on the order of a factor 3-10.

The situation for faint clusters is not as unambiguous, as shown in Fig. 6.31. However, the improvement is still visible, but somehwat harder to quantify.
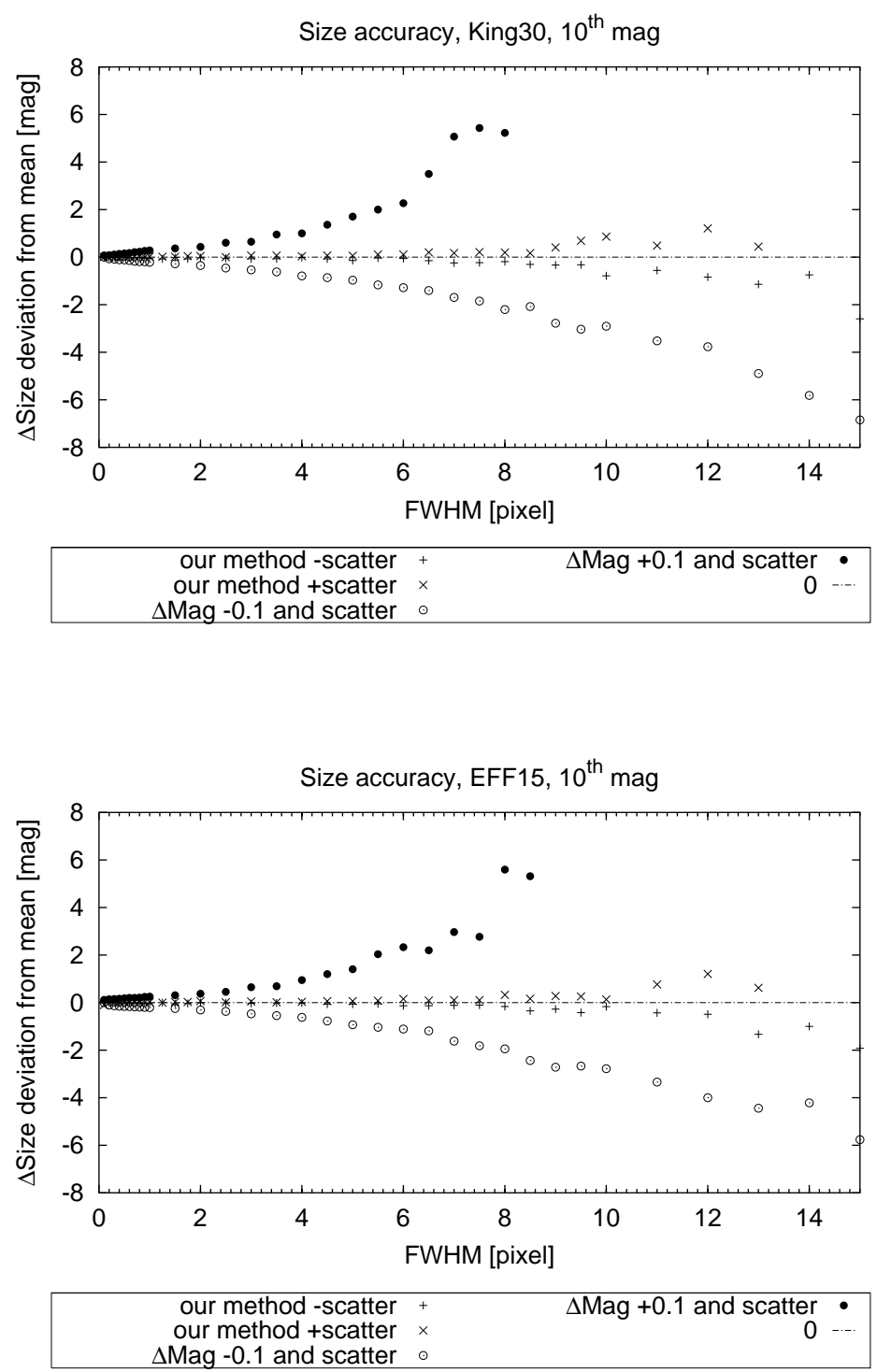

Figure 6.30: Scatter in the size determination from the DeltaMag method (assuming a photometric accuracy of $\pm 0.1 \mathrm{mag}$ ), compared to the scatter introduced by our method for a standard cluster. Top panel: King 30 profile. Bottom panel: EFF 15 profile. 

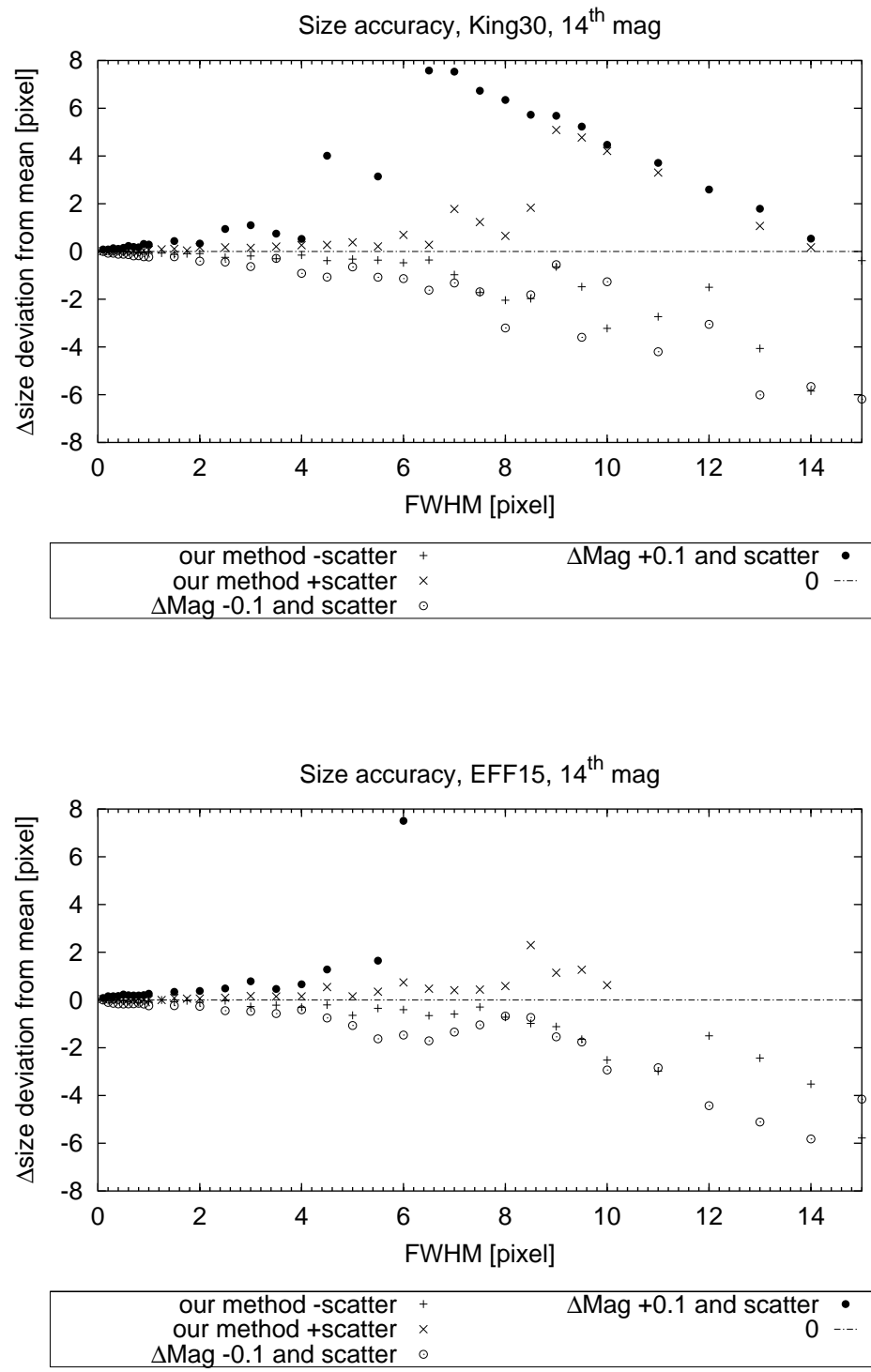

Figure 6.31: Scatter in the size determination from the DeltaMag method (assuming a photometric accuracy of $\pm 0.1 \mathrm{mag})$, compared to the scatter introduced by our method for a $V=14 \mathrm{mag}$ cluster. Top panel: King 30 profile. Bottom panel: EFF 15 profile.

\subsubsection{Aperture corrections}

As we have shown in the previous section, our size determination method represents a significant improvement compared to the widely used DeltaMag method. While this is important in its own right, the accuracy of the AC calculations (and hence the determination of reliable absolute magnitudes for extended spherically symmetric sources) is of even greater importance.

While the size uncertainties correlate directly with the $\mathrm{AC}$ uncertainties, 
because of the non-linearity of the ACs we give the $\mathrm{AC}$ uncertainties for a number of cases in Fig. 6.32. The improvement of our method with respect to the DeltaMag method is clearly seen. Quantitavely, the mean improvement represents a factor of $\sim 6-9$, covering a total range of 3-40. We emphasize that the uncertainties stated here for the DeltaMag method take into account only the uncertainty arising from a generic uncertainty in the magnitude determination of 0.1 mag. We take the $\mathrm{AC}$ relations determined in this paper, while there might be additional differences/uncertainties related to the DeltaMag as used in the papers cited, especially the mentioned centering problems.
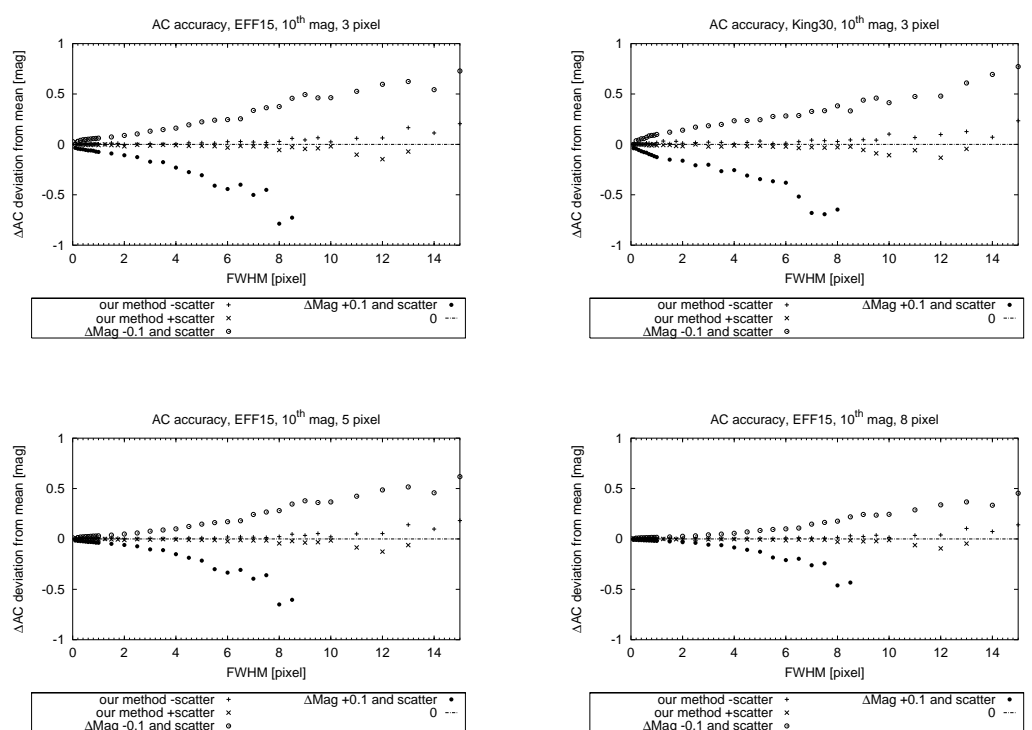

Figure 6.32: Scatter in the AC calculation from the DeltaMag method (assuming a photometric accuracy of $\pm 0.1 \mathrm{mag}$ ), compared to the scatter introduced by our method for a standard cluster. Top panels: 3 pixel radius apertures for an EFF 15 profile (left) and a King 30 profile (right). Bottom panels: Assuming an EFF 15 profile, and using 5 pixel (left) and 8 pixel (right) radius apertures.

\subsection{Summary}

We have presented an update to and significant improvement of the commonly used method of aperture photometry for HST imaging of extended circularly symmetric sources, including a reliable algorithm to determine accurate sizes of such objects.

Aperture photometry, by definition, underestimates the flux of any source if finite apertures are used. This is particularly relevant for HST imaging owing to the large extent of the PSFs, and the high spatial resolution, which makes small apertures possible and desirable to overcome crowding effects.

For this purpose, we investigated the possibilities to measure sizes of extended spherically symmetric objects accurately, and use this size information to obtain size-dependent ACs. This allows one to determine, in particular, 
masses of the objects based on integrated photometry more reliably, such as for extragalactic star clusters.

We modelled a large grid of artificial star clusters using a large range of input parameters, both intrinsic to the object (size, light profile, brightness, sky background) and observational (HST camera/chip, filter, position on the chip), using the BAOLAB package of Larsen (1999). This package provides the user with good flexibility and realistic modelling of cluster light profile observations.

We first established the relationship between input size of a cluster (in terms of the FWHM of its light profile) and the measured size in terms of the FWHM of a Gaussian profile fitted to a given cluster. Bi-directional polynomial relations between these input and output FWHMs were established and collected in Section 6.8.1.

In general, the differences between the results for different input parameters are only significant for (i) different input light profiles, (ii) different HST cameras, (iii) different fitting radii (maximum radius up to which the fit will be performed), (iv) (for NICMOS only) different observation epochs and filters, and (v) (for WFPC2 and ACS/WFC only) marginally significant for different filters and chips. Although we checked a large number of potentially important additional factors (such as, e.g., the exact position of an object on a certain chip, and the stellar spectrum used to create PSFs), we found the impact of those to be within the scatter introduced by the random effects inherent to cluster creation $(\sim \pm 0.2$ pixels $)$.

Using the information thus obtained, we determined ACs for the same clusters that we determined sizes for. In Sections 6.8.2 and 6.8.3 we present the results as a function of the intrinsic and the measured sizes of the clusters, respectively.

As an example of the importance of using proper ACs for extended spherically symmetric sources, assume that we observe a cluster with an effective radius of $3 \mathrm{pc}$, located at a distance of $5 \mathrm{Mpc}$. Depending on the details of the observations and the data analysis, neglecting these size-dependent ACs may underestimate the brightness of the cluster by $0.3-1.3 \mathrm{mag}$, corresponding to mass underestimates of $30-330 \%$.

In Section 6.4 we provide a cookbook for observers who aim to improve the accuracy of their aperture photometry of extended spherically symmetric objects.

\subsection{Acknowledgements}

The authors are grateful to the International Space Science Iinstitute in Bern (Switzerland) for their hospitality and research support, as part of an International Team programme. In addition, we thank Henny Lamers, Remco Scheepmaker and Uta Fritze-v. Alvensleben for useful discussions. PA is partially funded by DFG grant Fr 911/11-3. We thank the anonymous referee for many useful suggestions. 


\section{Bibliography}

Ashman, K. \& Zepf, S. 1998, Globular Cluster Systems (Cambridge University Press, Cambridge)

Binney, J. \& Tremaine, S. 1998, Galactic Dynamics (Princeton University Press, Princeton)

Boeker, T., Sarzi, M., McLaughlin, D. E., van der Marel, R. P., Rix, H.-W., Ho, L. C. \& Shields, J. C. 2004, AJ, 127, 105

Carlson, M. N., Holtzman, J. A., Watson, A. M., Grillmair, C. J., Mould, J. R., Ballester, G. E., Burrows, C. J. \& Clarke, J. T. 1998, AJ, 115, 1778

Carlson M. N. \& Holtzman J. A. 2001, PASP, 113, 1522

de Grijs, R., O'Connell, R. W. \& Gallagher III, J. S. 2001, AJ, 121, 768

Dolphin, A. E. 2000, PASP, 112, 1383

Dolphin, A. E., Kennicutt, R. C. 2002, AJ 123, 207

Elson, R. A. W., Fall, S. M. \& Freeman, K. C. 1987, ApJ, 323, 54

Holtzman, J. A., Burrows, C. J., Casertano, S., Hester, J. J., Trauger, J. T., Watson, A. M. \& Worthey, G. 1995, PASP, 107, 1065

King, I. 1962, AJ, 67, 471

Krist, J. \& Hook, R. 2004, The Tiny Tim User's Guide Version 6.3 (STScI, Baltimore)

Larsen, S. S. 1999, A\&AS, 139, 393

Larsen, S. S. 2004a, A\&A, 416, 537

Larsen, S. S. 2004b, An ISHAPE user's guide, http://www. astro.uu.nl/ larsen/

Mackey, A. D. \& Gilmore, G. F. 2003a, MNRAS, 338, 85

Mackey, A. D. \& Gilmore, G. F. 2003b, MNRAS, 340, 175

Miller, B. W., Whitmore, B. C., Schweizer, F. \& Fall, S. M. 1997, AJ, 114, 2381

Puzia, T. H., Kissler-Patig, M., Brodie, J. P. \& Huchra, J. P. 1999, AJ, 118, 2734 
Suchkov, A. \& Casertano, S. 1997, in: The 1997 HST Calibration Workshop with a new generation of instruments, ed. Casertano, S., Jedrzejewski, R., Keyes, C. D. \& Stevens, M. (STScI, Baltimore) 378

Whitmore, B. C., Schweizer, F., Leitherer, C., Borne, K. \& Robert, C. 1993, AJ, 106, 1354

Whitmore, B. C. \& Schweizer, F. 1995, AJ, 109, 960

Zepf, S. E., Ashman, K. M., English, J., Freeman, K. C. \& Sharples, R. M. 1999, AJ, 118, 752 


\subsection{Appendices}

\subsubsection{Parameters of cluster sizes fits}

In this subsection we present tables containing the fit parameters of our cluster size studies, starting with Table 6.4.

The fitting equations are

$$
\operatorname{size}(x)=a+b * x+c * x^{2}+d * x^{3}+e * x^{4}+f * x^{5}
$$

and

$$
\operatorname{size}^{\prime}(y)=a^{\prime}+b^{\prime} * y+c^{\prime} * y^{2}+d^{\prime} * y^{3}+e^{\prime} * y^{4}+f^{\prime} * y^{5}
$$

where $x$ and $\operatorname{size}^{\prime}(y)$ are the intrinsic FWHM in pixels, and $\operatorname{size}(x)$ and $y$ the measured FWHM.

For reference, we also give the sizes of the PSFs as such, measured using the same procedure as for the clusters (Table 6.3).

Table 6.3: The sizes of the PSFs as such, measured using the same procedure as for the clusters. "Pre" and "Post" refer to either before or after the installation of the NICMOS cryocooler in the year 2002.

\begin{tabular}{|c|c|c|c|}
\hline camera & epoch & filter & FWHM of PSF [pixel] \\
\hline ACS WFC & - & $B$ & 2.26 \\
ACS WFC & - & $V$ & 2.26 \\
ACS WFC & - & $I$ & 2.19 \\
NIC2 & post & $J$ & 1.46 \\
NIC2 & post & $H$ & 1.67 \\
NIC2 & post & $K$ & 2.02 \\
NIC2 & pre & $J$ & 3.61 \\
NIC2 & pre & $H$ & 1.75 \\
NIC2 & pre & $K$ & 2.07 \\
WFPC2 PC & - & $U$ & 1.70 \\
WFPC2 PC & - & $B$ & 1.98 \\
WFPC2 PC & - & $V$ & 2.12 \\
WFPC2 PC & - & $I$ & 1.84 \\
WFPC2 WF3 & - & $U$ & 1.91 \\
WFPC2 WF3 & - & $B$ & 1.91 \\
WFPC2 WF3 & - & $V$ & 1.91 \\
WFPC2 WF3 & - & $I$ & 2.08 \\
\hline
\end{tabular}


Table 6.4: Fit results of cluster sizes for a "standard" cluster. Upper panel: $\operatorname{Size}(x)$; Lower panel: $\operatorname{Size}^{\prime}(y)$.

\begin{tabular}{|c|c|c|c|c|c|c|}
\hline Profile & $a$ & $b$ & $c$ & $d$ & $e$ & $f$ \\
\hline King5 & 1.63787 & +1.03359 & -0.00500374 & -0.00943275 & +0.000963603 & $-2.74858 \mathrm{E}-05$ \\
King30 & 2.04549 & +1.52706 & -0.297628 & +0.0397823 & -0.00242798 & $+5.51152 \mathrm{E}-05$ \\
King100 & 2.32858 & +1.26613 & -0.220773 & +0.030224 & -0.00193542 & $+4.67765 \mathrm{E}-05$ \\
Moffat15 & 1.91591 & +1.18666 & -0.144827 & +0.0175979 & -0.00104467 & $+2.43509 \mathrm{E}-05$ \\
Moffat25 & 1.73853 & +0.873333 & -0.00606163 & -0.00245939 & +0.000253589 & $-6.36749 \mathrm{E}-06$ \\
\hline \hline Profile & $a^{\prime}$ & $b^{\prime}$ & $c^{\prime}$ & $d^{\prime}$ & $e^{\prime}$ & $f^{\prime}$ \\
\hline King5 & -3.68916 & +3.38959 & -0.997741 & +0.181195 & -0.0137356 & +0.000374692 \\
King30 & 1.98751 & -2.11849 & +0.668887 & -0.0351309 & -0.00107904 & +0.000109362 \\
King100 & 3.72011 & -4.16017 & +1.49836 & -0.189099 & +0.0123212 & -0.000330543 \\
Moffat15 & -0.243911 & -0.476292 & +0.387727 & -0.0341423 & +0.00154663 & $-3.25775 \mathrm{E}-05$ \\
Moffat25 & -2.12227 & +1.34245 & -0.10647 & +0.0244149 & -0.00188937 & $+4.78649 \mathrm{E}-05$ \\
\hline
\end{tabular}


Table 6.5: Fit results of cluster sizes for a "standard" cluster, using various filters: $U=\mathrm{F} 336 \mathrm{~W}, B=\mathrm{F} 439 \mathrm{~W}, \mathrm{H} \alpha=\mathrm{F} 656 \mathrm{~N}, I=\mathrm{F} 814 \mathrm{~W}$. See Sect. 6.2.7. Size $(x)$

\begin{tabular}{|c|c|c|c|c|c|c|c|}
\hline Profile & filter & $a$ & $b$ & $c$ & $d$ & $e$ & $f$ \\
\hline King 5 & $U$ & 1.57508 & +1.18233 & -0.0858621 & +0.00577574 & -0.000157559 & $+8.479 \mathrm{E}-07$ \\
King 5 & $B$ & 1.64425 & +0.974007 & +0.0318962 & -0.0172564 & +0.00164378 & $-4.78199 \mathrm{E}-05$ \\
King 5 & $\mathrm{H} \alpha$ & 1.75561 & +0.839707 & +0.0984069 & -0.0311313 & +0.0028463 & $-8.42084 \mathrm{E}-05$ \\
King 5 & $I$ & 1.75404 & +1.01829 & -0.0222498 & -0.00354398 & +0.000355291 & $-7.35525 \mathrm{E}-06$ \\
\hline King 30 & $U$ & 2.09919 & +1.35471 & -0.18394 & +0.0136512 & $-4.22014 \mathrm{E}-05$ & $-1.78826 \mathrm{E}-05$ \\
King 30 & $B$ & 2.00275 & +1.59272 & -0.327562 & +0.0450625 & -0.00281167 & $+6.53583 \mathrm{E}-05$ \\
King 30 & $\mathrm{H} \alpha$ & 1.94905 & +1.87942 & -0.514401 & +0.0855341 & -0.00627893 & +0.000166189 \\
King 30 & $I$ & 2.13881 & +1.51781 & -0.303502 & +0.0419262 & -0.00265883 & $+6.28841 \mathrm{E}-05$ \\
\hline King 100 & $U$ & 2.35767 & +1.105 & -0.112429 & +0.00567899 & +0.000283993 & $-2.16328 \mathrm{E}-05$ \\
King 100 & $B$ & 2.37172 & +1.10334 & -0.116229 & +0.00665969 & +0.000196686 & $-1.92439 \mathrm{E}-05$ \\
King 100 & $\mathrm{H} \alpha$ & 2.43776 & +1.05049 & -0.10156 & +0.00517682 & +0.000249325 & $-2.01187 \mathrm{E}-05$ \\
King 100 & $I$ & 2.47061 & +1.13988 & -0.162235 & +0.0189728 & -0.00100906 & $+1.98086 \mathrm{E}-05$ \\
\hline EFF 15 & $U$ & 2.04065 & +0.800232 & +0.100454 & -0.0372574 & +0.0038787 & -0.000126805 \\
EFF 15 & $B$ & 1.86521 & +1.28482 & -0.196804 & +0.0274572 & -0.00181691 & $+4.57589 \mathrm{E}-05$ \\
EFF 15 & $\mathrm{H} \alpha$ & 1.83848 & +1.43384 & -0.293639 & +0.0475078 & -0.00338408 & $+8.59167 \mathrm{E}-05$ \\
EFF 15 & $I$ & 2.03165 & +1.1371 & -0.131187 & +0.0155285 & -0.000884015 & $+1.95425 \mathrm{E}-05$ \\
\hline EFF 25 & $U$ & 1.75267 & +0.86926 & -0.00379334 & -0.00272783 & +0.000247273 & $-5.19432 \mathrm{E}-06$ \\
EFF 25 & $B$ & 1.74017 & +0.825105 & +0.0202964 & -0.00776042 & +0.000707637 & $-1.97666 \mathrm{E}-05$ \\
EFF 25 & $\mathrm{H} \alpha$ & 1.86276 & +0.613448 & +0.153041 & -0.0387789 & +0.003641 & -0.000115622 \\
EFF 25 & $I$ & 1.90252 & +0.729092 & +0.0532549 & -0.0129125 & +0.00105149 & $-2.79848 \mathrm{E}-05$ \\
\hline
\end{tabular}


Table 6.6: Fit results of cluster sizes for a "standard" cluster, using various filters: $U=\mathrm{F} 336 \mathrm{~W}, B=\mathrm{F} 439 \mathrm{~W}, \mathrm{H} \alpha=\mathrm{F} 656 \mathrm{~N}, I=\mathrm{F} 814 \mathrm{~W}$. See Sect. 6.2.7. $\operatorname{Size}^{\prime}(y)$.

\begin{tabular}{|c|c|c|c|c|c|c|c|}
\hline Profile & filter & $a^{\prime}$ & $b^{\prime}$ & $c^{\prime}$ & $d^{\prime}$ & $e^{\prime}$ & $f^{\prime}$ \\
\hline King 5 & $U$ & -2.37095 & +2.00573 & -0.483838 & +0.0964808 & -0.00742288 & +0.000199818 \\
King 5 & $B$ & -4.52674 & +4.3485 & -1.38498 & +0.25206 & -0.0197085 & +0.000561245 \\
King 5 & $\mathrm{H} \alpha$ & 1.18706 & -2.16984 & +1.27335 & -0.239944 & +0.0222174 & -0.000766828 \\
King 5 & $I$ & -2.90574 & +2.301 & -0.526329 & +0.0919896 & -0.00613695 & +0.000136093 \\
\hline King 30 & $U$ & 6.38681 & -6.90997 & +2.58562 & -0.389224 & +0.0294628 & -0.000886395 \\
King 30 & $B$ & 2.49002 & -2.68914 & +0.91274 & -0.083502 & +0.00341804 & $-5.11725 \mathrm{E}-05$ \\
King 30 & $\mathrm{H} \alpha$ & 8.96857 & -9.67051 & +3.65704 & -0.5793 & +0.0449362 & -0.0013485 \\
King 30 & $I$ & 3.24724 & -3.41016 & +1.14835 & -0.120746 & +0.00622088 & -0.000127111 \\
\hline King 100 & $U$ & 8.26542 & -8.86481 & +3.32396 & -0.520068 & +0.0404879 & -0.00123726 \\
King 100 & $B$ & -6.56816 & +6.71665 & -2.7983 & +0.605177 & -0.0567013 & +0.00193783 \\
King 100 & $\mathrm{H} \alpha$ & 15.9582 & -16.9197 & +6.46104 & -1.09126 & +0.0892864 & -0.00280226 \\
King 100 & $I$ & 1.05227 & -1.39849 & +0.403392 & +0.0127654 & -0.00510762 & +0.000237123 \\
\hline EFF 15 & $U$ & 5.53267 & -6.73679 & +2.87436 & -0.487665 & +0.0398467 & -0.00124028 \\
EFF 15 & $B$ & -0.230254 & -0.468754 & +0.382237 & -0.0333104 & +0.00158066 & $-3.95014 \mathrm{E}-05$ \\
EFF 15 & $\mathrm{H} \alpha$ & -0.335385 & -0.526631 & +0.460188 & -0.0549301 & +0.00377868 & -0.00011302 \\
EFF 15 & $I$ & -0.325479 & -0.4707 & +0.378585 & -0.0305488 & +0.00107651 & $-1.27583 \mathrm{E}-05$ \\
\hline EFF 25 & $U$ & -2.36881 & +1.57655 & -0.188774 & +0.036949 & -0.00271951 & $+6.71307 \mathrm{E}-05$ \\
EFF 25 & $B$ & -2.46935 & +1.72432 & -0.248578 & +0.0485995 & -0.00380523 & +0.00010385 \\
EFF 25 & $\mathrm{H} \alpha$ & 2.39599 & -3.69551 & +1.90434 & -0.338795 & +0.0281665 & -0.000872747 \\
EFF 25 & $I$ & -3.34986 & +2.3763 & -0.449016 & +0.0773671 & -0.00570389 & +0.000150732 \\
\hline
\end{tabular}


Table 6.7: Fit results of cluster sizes for a "standard" cluster, using various WFPC2 chips. See Section 6.2.7. Size(x).

\begin{tabular}{|c|c|c|c|c|c|c|c|}
\hline Profile & WFPC2 chip & $a$ & $b$ & $c$ & $d$ & $e$ & $f$ \\
\hline King 5 & PC & 1.834 & +0.8782 & +0.0516633 & -0.0181559 & +0.00157149 & $-4.30532 \mathrm{E}-05$ \\
King 5 & WF2 & 1.37031 & +1.1469 & -0.038673 & -0.00419889 & +0.00057642 & $-1.685 \mathrm{E}-05$ \\
King 5 & WF4 & 1.40963 & +1.14354 & -0.0347369 & -0.00544424 & +0.000710431 & $-2.13276 \mathrm{E}-05$ \\
\hline King 30 & PC & 2.14599 & +1.50923 & -0.296254 & +0.040757 & -0.00259625 & $+6.22677 \mathrm{E}-05$ \\
King 30 & WF2 & 1.77065 & +1.6273 & -0.319273 & +0.0420499 & -0.00254481 & $+5.74474 \mathrm{E}-05$ \\
King 30 & WF4 & 1.85451 & +1.64476 & -0.339053 & +0.0465527 & -0.00294066 & $+6.96077 \mathrm{E}-05$ \\
\hline King 100 & PC & 2.45192 & +1.18406 & -0.181658 & +0.0230013 & -0.0013694 & $+3.11742 \mathrm{E}-05$ \\
King 100 & WF2 & 2.06047 & +1.37026 & -0.25274 & +0.0354982 & -0.00235001 & $+5.88236 \mathrm{E}-05$ \\
King 100 & WF4 & 2.16075 & +1.33995 & -0.243863 & +0.0336598 & -0.00216489 & $+5.22736 \mathrm{E}-05$ \\
\hline EFF 15 & PC & 2.08037 & +1.05347 & -0.0899971 & +0.0084123 & -0.000378886 & $+7.12857 \mathrm{E}-06$ \\
EFF 15 & WF2 & 1.647 & +1.26864 & -0.161382 & +0.0193808 & -0.00113739 & $+2.59554 \mathrm{E}-05$ \\
EFF 15 & WF4 & 1.72618 & +1.24742 & -0.149455 & +0.0161944 & -0.000820518 & $+1.5942 \mathrm{E}-05$ \\
\hline EFF 25 & PC & 1.92252 & +0.759304 & +0.0238015 & -0.00497828 & +0.00027424 & $-2.97158 \mathrm{E}-06$ \\
EFF 25 & WF2 & 1.48987 & +0.915657 & -0.00530929 & -0.00330809 & +0.000314744 & $-7.42061 \mathrm{E}-06$ \\
EFF 25 & WF4 & 1.52263 & +0.949646 & -0.0209295 & -0.000773582 & +0.000141743 & $-3.21985 \mathrm{E}-06$ \\
\hline
\end{tabular}


Table 6.8: Fit results of cluster sizes for a "standard" cluster, using various WFPC2 chips. See Section 6.2.7. Size'(y).

\begin{tabular}{|c|c|c|c|c|c|c|c|}
\hline Profile & WFPC2 chip & $a^{\prime}$ & $b^{\prime}$ & $c^{\prime}$ & $d^{\prime}$ & $e^{\prime}$ & $f^{\prime}$ \\
\hline King 5 & PC & -4.9441 & +4.3424 & -1.27585 & +0.218679 & -0.0161489 & +0.000434474 \\
King 5 & WF2 & -2.60677 & +2.58009 & -0.731604 & +0.139347 & -0.0105826 & +0.000283316 \\
King 5 & WF4 & -2.73066 & +2.68978 & -0.781901 & +0.149523 & -0.0115164 & +0.000314393 \\
\hline King 30 & PC & 2.91244 & -3.10097 & +1.05447 & -0.110106 & +0.00586997 & -0.000133769 \\
King 30 & WF2 & 0.999229 & -1.16256 & +0.384449 & +0.00417507 & -0.00363655 & +0.000173122 \\
King 30 & WF4 & 1.91289 & -2.15729 & +0.759824 & -0.0631674 & +0.00216637 & $-2.02405 \mathrm{E}-05$ \\
\hline King 100 & PC & 2.07719 & -2.48964 & +0.850315 & -0.0745911 & +0.00289527 & $-3.85914 \mathrm{E}-05$ \\
King 100 & WF2 & 2.81198 & -3.4139 & +1.34202 & -0.179798 & +0.012785 & -0.000377525 \\
King 100 & WF4 & 3.22223 & -3.73106 & +1.39343 & -0.177276 & +0.0116713 & -0.000315518 \\
\hline EFF 15 & PC & -1.61186 & +0.721619 & -0.0224735 & +0.0304737 & -0.00320717 & $+9.91342 \mathrm{E}-05$ \\
EFF 15 & WF2 & -0.449999 & -0.0804472 & +0.252982 & -0.0136059 & $+5.95403 \mathrm{E}-05$ & $+9.76978 \mathrm{E}-06$ \\
EFF 15 & WF4 & -0.775498 & +0.293966 & +0.0689952 & +0.0249565 & -0.00346238 & +0.000124382 \\
\hline EFF 25 & PC & -2.8778 & +1.77716 & -0.194971 & +0.0299973 & -0.00177603 & $+3.25511 \mathrm{E}-05$ \\
EFF 25 & WF2 & -1.84817 & +1.3918 & -0.147166 & +0.0316476 & -0.00238108 & $+5.90178 \mathrm{E}-05$ \\
EFF 25 & WF4 & -1.70264 & +1.19897 & -0.0838419 & +0.0224247 & -0.00176533 & $+4.37257 \mathrm{E}-05$ \\
\hline
\end{tabular}


Table 6.9: Fit results of cluster sizes for a "standard" cluster, using various filters, and using the appropriate PSF for the fitting: $U=$ F336W, $B=$ F439W, $V=$ F555W, $I=$ F814W. See Section 6.2.6. Size $(x)$.

\begin{tabular}{|c|c|c|c|c|c|c|c|}
\hline Profile & filter & $a$ & $b$ & $c$ & $d$ & $e$ & $f$ \\
\hline King 5 & U & 0.626241 & +1.14726 & +0.0760287 & -0.0356841 & +0.00356999 & -0.000110118 \\
King 5 & B & 0.598337 & +1.25728 & -0.00142682 & -0.0173499 & +0.0018741 & $-5.74757 \mathrm{E}-05$ \\
King 5 & V & 0.584621 & +1.30494 & -0.0307285 & -0.0109286 & +0.00130184 & $-4.01726 \mathrm{E}-05$ \\
King 5 & I & 0.708369 & +1.15012 & +0.0260514 & -0.0194617 & +0.0018648 & $-5.34443 \mathrm{E}-05$ \\
\hline King 30 & U & 0.99394 & +2.11079 & -0.517682 & +0.0783347 & -0.00541312 & +0.000138122 \\
King 30 & B & 1.10137 & +1.82456 & -0.341293 & +0.0400241 & -0.00209565 & $+3.99217 \mathrm{E}-05$ \\
King 30 & V & 1.05176 & +1.95781 & -0.422087 & +0.057762 & -0.00366087 & $+8.68349 \mathrm{E}-05$ \\
King 30 & I & 1.08356 & +1.93943 & -0.422052 & +0.0589365 & -0.00381994 & $+9.26919 \mathrm{E}-05$ \\
\hline King 100 & U & 1.38831 & +1.65649 & -0.340315 & +0.0483316 & -0.00318673 & $+7.80256 \mathrm{E}-05$ \\
King 100 & B & 1.48554 & +1.39072 & -0.173062 & +0.0111674 & +0.000110388 & $-2.14461 \mathrm{E}-05$ \\
King 100 & V & 1.41676 & +1.59333 & -0.296696 & +0.0385753 & -0.00234998 & $+5.41087 \mathrm{E}-05$ \\
King 100 & I & 1.43588 & +1.58938 & -0.299049 & +0.0394417 & -0.00243009 & $+5.63027 \mathrm{E}-05$ \\
\hline EFF 15 & U & 0.861043 & +1.63729 & -0.275085 & +0.0355667 & -0.00216707 & $+5.03278 \mathrm{E}-05$ \\
EFF 15 & B & 0.917945 & +1.50731 & -0.204895 & +0.0221952 & -0.00112705 & $+2.19351 \mathrm{E}-05$ \\
EFF 15 & V & 0.923924 & +1.50152 & -0.207219 & +0.0235847 & -0.00129759 & $+2.77089 \mathrm{E}-05$ \\
EFF 15 & I & 0.977115 & +1.43822 & -0.183721 & +0.0200114 & -0.00106031 & $+2.20222 \mathrm{E}-05$ \\
\hline EFF 25 & U & 0.640607 & +1.23807 & -0.092612 & +0.00939997 & -0.000557649 & $+1.48049 \mathrm{E}-05$ \\
EFF 25 & B & 0.744147 & +0.991961 & +0.0480464 & -0.0201357 & +0.00197459 & $-6.04627 \mathrm{E}-05$ \\
EFF 25 & V & 0.719956 & +1.06776 & -0.000338317 & -0.00901312 & +0.000948061 & $-2.82741 \mathrm{E}-05$ \\
EFF 25 & I & 0.858481 & +0.896448 & +0.0669991 & -0.0202957 & +0.00179173 & $-5.1317 \mathrm{E}-05$ \\
\hline
\end{tabular}


Table 6.10: Fit results of cluster sizes for a "standard" cluster, using various filters, and using the appropriate PSF for the fitting: $U=$ F336W, $B=\mathrm{F} 439 \mathrm{~W}, V=\mathrm{F} 555 \mathrm{~W}, I=\mathrm{F} 814 \mathrm{~W}$. See Section 6.2.6. $\operatorname{Size}^{\prime}(y)$.

\begin{tabular}{|c|c|c|c|c|c|c|c|}
\hline Profile & filter & $a^{\prime}$ & $b^{\prime}$ & $c^{\prime}$ & $d^{\prime}$ & $e^{\prime}$ & $f^{\prime}$ \\
\hline King 5 & $\mathrm{U}$ & -0.823697 & +1.46008 & -0.396536 & +0.0941689 & -0.00781587 & +0.000218535 \\
King 5 & $\mathrm{B}$ & -1.37261 & +2.30831 & -0.81674 & +0.182011 & -0.0159362 & +0.000492332 \\
King 5 & $\mathrm{V}$ & -1.23964 & +2.08172 & -0.695095 & +0.154693 & -0.0132704 & +0.000400412 \\
King 5 & $\mathrm{I}$ & -1.49006 & +2.29979 & -0.757104 & +0.161739 & -0.0135481 & +0.000399412 \\
\hline King 30 & $\mathrm{U}$ & 1.34903 & -1.98484 & +1.02296 & -0.155978 & +0.012724 & -0.000417655 \\
King 30 & $\mathrm{B}$ & -0.438138 & +0.743334 & -0.371267 & +0.149493 & -0.016846 & +0.000619894 \\
King 30 & $\mathrm{V}$ & 0.413502 & -0.573322 & +0.314557 & -0.0044935 & -0.00158012 & $+7.48153 \mathrm{E}-05$ \\
King 30 & $\mathrm{I}$ & 0.679758 & -0.97138 & +0.513635 & -0.0480248 & +0.00266561 & $-7.66904 \mathrm{E}-05$ \\
\hline King 100 & $\mathrm{U}$ & 1.25928 & -1.86828 & +0.918206 & -0.124263 & +0.00907347 & -0.000271056 \\
King 100 & $\mathrm{B}$ & 1.57409 & -2.30185 & +1.13058 & -0.172274 & +0.0141727 & -0.000476239 \\
King 100 & $\mathrm{V}$ & 0.429801 & -0.723849 & +0.371332 & -0.0111514 & -0.00130611 & $+7.35518 \mathrm{E}-05$ \\
King 100 & $\mathrm{I}$ & 0.567917 & -0.917423 & +0.459115 & -0.0283514 & +0.000153575 & $+2.99008 \mathrm{E}-05$ \\
\hline EFF 15 & $\mathrm{U}$ & -0.0772117 & +0.00291678 & +0.22503 & -0.00739615 & -0.00065604 & $+3.59012 \mathrm{E}-05$ \\
EFF 15 & $\mathrm{B}$ & -0.458033 & +0.513791 & -0.0018533 & +0.0351584 & -0.00420464 & +0.000145017 \\
EFF 15 & $\mathrm{V}$ & -0.378241 & +0.377702 & +0.0741028 & +0.0177505 & -0.00252258 & $+8.91963 \mathrm{E}-05$ \\
EFF 15 & $\mathrm{I}$ & -0.4827 & +0.474321 & +0.0404885 & +0.0230756 & -0.00291479 & $+9.99763 \mathrm{E}-05$ \\
\hline EFF 25 & $\mathrm{U}$ & -0.442966 & +0.678778 & +0.0726838 & -0.00230767 & $+7.98127 \mathrm{E}-05$ & $-8.07776 \mathrm{E}-06$ \\
EFF 25 & $\mathrm{B}$ & -1.13082 & +1.7194 & -0.436592 & +0.102309 & -0.00930249 & +0.000294766 \\
EFF 25 & $\mathrm{V}$ & -0.850378 & +1.27088 & -0.206106 & +0.0525278 & -0.00462614 & +0.00013799 \\
EFF 25 & $\mathrm{I}$ & -1.26898 & +1.71339 & -0.373709 & +0.0815885 & -0.00697307 & +0.000209205 \\
\hline
\end{tabular}


Table 6.11: Fit results of cluster sizes for a "standard" cluster, using various filters available for the ACS/WFC (chip 1, except V2): $B$ $=\mathrm{F} 435 \mathrm{~W}, V=\mathrm{F} 555 \mathrm{~W}, V 2=\mathrm{F} 555 \mathrm{~W}$ chip2, $I=\mathrm{F} 814 \mathrm{~W}$. See Section 6.2.8. $\operatorname{Size}(x)$.

\begin{tabular}{|c|c|c|c|c|c|c|c|}
\hline Profile & filter & $a$ & $b$ & $c$ & $d$ & $e$ & $f$ \\
\hline King 5 & B & 2.01448 & +0.788272 & +0.0729654 & -0.021073 & +0.00176827 & $-4.821 \mathrm{E}-05$ \\
King 5 & V1 & 1.99947 & +0.781468 & +0.0786108 & -0.0223954 & +0.00188616 & $-5.17918 \mathrm{E}-05$ \\
King 5 & V2 & 1.93114 & +0.826626 & +0.0677426 & -0.0210485 & +0.00180356 & $-4.98126 \mathrm{E}-05$ \\
King 5 & I & 1.9654 & +1.10136 & -0.0541613 & -0.00101662 & +0.000399461 & $-1.43918 \mathrm{E}-05$ \\
\hline King 30 & B & 2.34391 & +1.35948 & -0.247975 & +0.033417 & -0.00208355 & $+4.87345 \mathrm{E}-05$ \\
King 30 & V1 & 2.30041 & +1.39505 & -0.262473 & +0.0358297 & -0.00225918 & $+5.33683 \mathrm{E}-05$ \\
King 30 & V2 & 2.23853 & +1.44434 & -0.275753 & +0.0374628 & -0.00234784 & $+5.50583 \mathrm{E}-05$ \\
King 30 & I & 2.42163 & +1.56772 & -0.341185 & +0.0484718 & -0.00311365 & $+7.40721 \mathrm{E}-05$ \\
\hline King 100 & B & 2.61057 & +1.11275 & -0.169898 & +0.0222714 & -0.00136573 & $+3.16435 \mathrm{E}-05$ \\
King 100 & V1 & 2.54219 & +1.15326 & -0.183659 & +0.0244589 & -0.0015256 & $+3.59537 \mathrm{E}-05$ \\
King 100 & V2 & 2.53632 & +1.13827 & -0.172688 & +0.0221398 & -0.00133082 & $+3.03102 \mathrm{E}-05$ \\
King 100 & I & 2.83101 & +1.11688 & -0.184875 & +0.0251716 & -0.00157262 & $+3.67312 \mathrm{E}-05$ \\
\hline EFF 15 & B & 2.26921 & +0.960306 & -0.0699971 & +0.00607077 & -0.000237451 & $+3.4729 \mathrm{E}-06$ \\
EFF 15 & V1 & 2.22594 & +0.983086 & -0.0781557 & +0.00735995 & -0.000323782 & $+5.46381 \mathrm{E}-06$ \\
EFF 15 & V2 & 2.15126 & +1.03689 & -0.0922518 & +0.0091314 & -0.000427844 & $+7.71942 \mathrm{E}-06$ \\
EFF 15 & I & 2.33482 & +1.21118 & -0.183044 & +0.0244413 & -0.00150142 & $+3.4659 \mathrm{E}-05$ \\
\hline EFF 25 & B & 2.13315 & +0.613487 & +0.0804595 & -0.0160308 & +0.0012265 & $-3.22813 \mathrm{E}-05$ \\
EFF 25 & V1 & 2.10732 & +0.627975 & +0.0689911 & -0.0132261 & +0.000968816 & $-2.43347 \mathrm{E}-05$ \\
EFF 25 & V2 & 2.03724 & +0.657218 & +0.0686817 & -0.0141831 & +0.00108607 & $-2.8308 \mathrm{E}-05$ \\
EFF 25 & I & 2.06146 & +0.974887 & -0.0671465 & +0.00740031 & -0.000385528 & $+7.9275 \mathrm{E}-06$ \\
\hline
\end{tabular}


Table 6.12: Fit results of cluster sizes for a "standard" cluster, using various filters available for the ACS/WFC (chip 1, except V2): $B$ $=\mathrm{F} 435 \mathrm{~W}, V=\mathrm{F} 555 \mathrm{~W}, V 2=\mathrm{F} 555 \mathrm{~W}$ chip2, $I=\mathrm{F} 814 \mathrm{~W}$. See Section 6.2.8. $\operatorname{Size}^{\prime}(y)$.

\begin{tabular}{|c|c|c|c|c|c|c|c|}
\hline Profile & filter & $a^{\prime}$ & $b^{\prime}$ & $c^{\prime}$ & $d^{\prime}$ & $e^{\prime}$ & $f^{\prime}$ \\
\hline King 5 & B & -6.38068 & +5.4792 & -1.64868 & +0.278461 & -0.020721 & +0.000568284 \\
King 5 & V1 & -6.58601 & +5.74205 & -1.75869 & +0.299067 & -0.0224762 & +0.000623729 \\
King 5 & V2 & -6.11431 & +5.40882 & -1.66229 & +0.284834 & -0.0214348 & +0.000593912 \\
King 5 & I & -4.05858 & +3.35084 & -0.97705 & +0.178915 & -0.0137156 & +0.000379729 \\
\hline King 30 & B & 3.60747 & -3.71827 & +1.22262 & -0.129041 & +0.00668707 & -0.000137868 \\
King 30 & V1 & 3.74182 & -3.88544 & +1.29857 & -0.143694 & +0.00796956 & -0.00017971 \\
King 30 & V2 & 3.15038 & -3.28054 & +1.08119 & -0.10772 & +0.00514641 & $-9.47236 \mathrm{E}-05$ \\
King 30 & I & 5.70517 & -5.30438 & +1.60947 & -0.171526 & +0.00877437 & -0.000173163 \\
\hline King 100 & B & 3.92803 & -4.28291 & +1.45556 & -0.168979 & +0.00982557 & -0.000230327 \\
King 100 & V1 & 4.08386 & -4.44152 & +1.53137 & -0.184807 & +0.0113116 & -0.000281671 \\
King 100 & V2 & 2.93253 & -3.33537 & +1.13677 & -0.118652 & +0.00604581 & -0.000121598 \\
King 100 & I & 7.83949 & -7.47286 & +2.37132 & -0.293601 & +0.0179659 & -0.000435892 \\
\hline EFF 15 & B & -1.74038 & +0.619316 & +0.030607 & +0.0224725 & -0.00273617 & $+9.14835 \mathrm{E}-05$ \\
EFF 15 & V1 & -1.42639 & +0.372216 & +0.110531 & +0.0106045 & -0.00193302 & $+7.16696 \mathrm{E}-05$ \\
EFF 15 & V2 & -1.43978 & +0.491768 & +0.0525169 & +0.021035 & -0.00275883 & $+9.6041 \mathrm{E}-05$ \\
EFF 15 & I & 2.54684 & -3.06783 & +1.1357 & -0.132075 & +0.00751315 & -0.000168777 \\
\hline EFF 25 & B & -4.77047 & +3.30605 & -0.699199 & +0.110449 & -0.00785708 & +0.000206949 \\
EFF 25 & V1 & -4.16032 & +2.73295 & -0.492318 & +0.0762673 & -0.00525235 & +0.000132794 \\
EFF 25 & V2 & -4.05799 & +2.79768 & -0.545958 & +0.0876559 & -0.0062264 & +0.000162247 \\
EFF 25 & I & -1.29234 & +0.231528 & +0.235909 & -0.0210673 & +0.000854926 & $-1.28966 \mathrm{E}-05$ \\
\hline
\end{tabular}


Table 6.13: Fit results of cluster sizes for a "standard" cluster, using various filters available for the NICMOS camera NIC2 $(J=$ F110W $H=\mathrm{F} 160 \mathrm{~W}, K=\mathrm{F} 205 \mathrm{~W}$ ) and 2 different epochs of observation ("1st Feb 1998" shows severe blurring due to focus shifts in the pre-cooler phase, which is not present for all pre-service mission observations; "late 2002" is an in-focus observation in the post-cooler phase.). See Section 6.2.9. $\operatorname{Size}(x)$.

\begin{tabular}{|c|c|c|c|c|c|c|c|c|}
\hline Profile & filter & epoch & $a$ & $b$ & $c$ & $d$ & $e$ & $f$ \\
\hline King 5 & $J$ & 1st Feb 1998 & 3.42735 & +0.171017 & +0.241217 & -0.0422611 & +0.00300925 & $-7.54603 \mathrm{E}-05$ \\
King 5 & $H$ & 1st Feb 1998 & 1.24955 & +2.59003 & -0.573376 & +0.0759222 & -0.00467052 & +0.000108104 \\
King 5 & $K$ & 1st Feb 1998 & 1.61338 & +1.63012 & -0.151631 & +0.00706783 & +0.000135725 & $-1.26806 \mathrm{E}-05$ \\
King 5 & $J$ & late 2002 & 1.19847 & +1.67495 & -0.221337 & +0.0203922 & -0.000803135 & $+9.86969 \mathrm{E}-06$ \\
King 5 & $H$ & late 2002 & 1.28037 & +2.16768 & -0.419151 & +0.0520928 & -0.0030446 & $+6.79259 \mathrm{E}-05$ \\
King 5 & $K$ & late 2002 & 1.55064 & +1.77438 & -0.226669 & +0.0194217 & -0.000722566 & $+8.88326 \mathrm{E}-06$ \\
\hline King 30 & $J$ & 1st Feb 1998 & 3.48673 & +1.04823 & -0.177483 & +0.0259506 & -0.00172046 & $+4.23514 \mathrm{E}-05$ \\
King 30 & $H$ & 1st Feb 1998 & 2.66934 & +2.31396 & -0.656922 & +0.100341 & -0.00676178 & +0.000166339 \\
King 30 & $K$ & 1st Feb 1998 & 2.11577 & +2.45415 & -0.623861 & +0.0891238 & -0.00574726 & +0.000136859 \\
King 30 & $J$ & late 2002 & 1.99335 & +1.85414 & -0.435839 & +0.0627486 & -0.00406822 & $+9.72531 \mathrm{E}-05$ \\
King 30 & $H$ & late 2002 & 2.34397 & +2.22883 & -0.617923 & +0.0941095 & -0.00635373 & +0.000156954 \\
King 30 & $K$ & late 2002 & 2.14241 & +2.4551 & -0.647411 & +0.0940174 & -0.00612809 & +0.000147074 \\
\hline King 100 & $J$ & 1st Feb 1998 & 3.7872 & +0.745909 & -0.0748029 & +0.0106386 & -0.000702977 & $+1.74376 \mathrm{E}-05$ \\
King 100 & $H$ & 1st Feb 1998 & 3.33926 & +1.45743 & -0.3396 & +0.0516825 & -0.00349713 & $+8.68089 \mathrm{E}-05$ \\
King 100 & $K$ & 1st Feb 1998 & 2.50418 & +1.99338 & -0.458839 & +0.0641793 & -0.00408947 & $+9.66507 \mathrm{E}-05$ \\
King 100 & $J$ & late 2002 & 2.45325 & +1.33541 & -0.254351 & +0.0357295 & -0.00229784 & $+5.49717 \mathrm{E}-05$ \\
King 100 & $H$ & late 2002 & 3.01541 & +1.3986 & -0.306656 & +0.0450548 & -0.0029531 & $+7.12782 \mathrm{E}-05$ \\
King 100 & $K$ & late 2002 & 2.57607 & +1.93738 & -0.455799 & +0.0639293 & -0.0040519 & $+9.51792 \mathrm{E}-05$ \\
\hline
\end{tabular}


Table 6.14: Fit results of cluster sizes for a "standard" cluster, using various filters available for the NICMOS camera NIC2 $(J=$ F110W $H=\mathrm{F} 160 \mathrm{~W}, K=\mathrm{F} 205 \mathrm{~W}$ ) and 2 different epochs of observation ("1st Feb 1998" shows severe blurring due to focus shifts in the pre-cooler phase, which is not present for all pre-service mission observations; "late 2002" is an in-focus observation in the post-cooler phase.). See Section 6.2.9. $\operatorname{Size}(x)$.

\begin{tabular}{|c|c|c|c|c|c|c|c|c|}
\hline Profile & filter & epoch & $a$ & $b$ & $c$ & $d$ & $e$ & $f$ \\
\hline EFF 15 & $J$ & 1st Feb 1998 & 3.54826 & +0.520179 & +0.0403598 & -0.00725457 & +0.000520385 & $-1.27074 \mathrm{E}-05$ \\
EFF 15 & $H$ & 1st Feb 1998 & 2.05163 & +2.49506 & -0.68321 & +0.103567 & -0.00695488 & +0.00017085 \\
EFF 15 & $K$ & 1st Feb 1998 & 1.8832 & +1.97531 & -0.384251 & +0.0500191 & -0.00303299 & $+6.94111 \mathrm{E}-05$ \\
EFF 15 & $J$ & late 2002 & 1.70962 & +1.6781 & -0.336456 & +0.0473722 & -0.00304491 & $+7.29309 \mathrm{E}-05$ \\
EFF 15 & $H$ & late 2002 & 1.90983 & +2.16645 & -0.542662 & +0.0798756 & -0.00523998 & +0.000126416 \\
EFF 15 & $K$ & late 2002 & 1.862 & +2.07163 & -0.448982 & +0.0620215 & -0.00394645 & $+9.41238 \mathrm{E}-05$ \\
\hline EFF 25 & $J$ & 1st Feb 1998 & 3.48284 & +0.142952 & +0.190267 & -0.0283099 & +0.00185847 & $-4.39851 \mathrm{E}-05$ \\
EFF 25 & $H$ & 1st Feb 1998 & 1.35563 & +2.48009 & -0.598934 & +0.0863637 & -0.00557814 & +0.000132865 \\
EFF 25 & $K$ & 1st Feb 1998 & 1.72443 & +1.41388 & -0.12885 & +0.0097986 & -0.000260631 & $-2.12114 \mathrm{E}-08$ \\
EFF 25 & $J$ & late 2002 & 1.3162 & +1.51023 & -0.226922 & +0.0289617 & -0.00169361 & $+3.75001 \mathrm{E}-05$ \\
EFF 25 & $H$ & late 2002 & 1.36869 & +2.05898 & -0.437658 & +0.0610983 & -0.00387192 & $+9.19997 \mathrm{E}-05$ \\
EFF 25 & $K$ & late 2002 & 1.64192 & +1.61373 & -0.224484 & +0.0250217 & -0.00129232 & $+2.55324 \mathrm{E}-05$ \\
\hline
\end{tabular}


Table 6.15: Fit results of cluster sizes for a "standard" cluster, using various filters available for the NICMOS camera NIC2 $(J=$ F110W, $H=$ F160W, $K=\mathrm{F} 205 \mathrm{~W}$ ) and 2 different epochs of observation ("1st Feb 1998" shows severe blurring due to focus shifts in the pre-cooler phase, which is not present for all pre-service mission observations; "late 2002" is an in-focus observation in the post-cooler phase.). See Section 6.2.9. Size'(y).

\begin{tabular}{|c|c|c|c|c|c|c|c|c|}
\hline Profile & filter & $a^{\prime}$ & $b^{\prime}$ & $c^{\prime}$ & $d^{\prime}$ & $e^{\prime}$ & $f^{\prime}$ & \\
\hline King 5 & $J$ & 1st Feb 1998 & -16.1168 & +10.3303 & -2.56526 & +0.34569 & -0.0217599 & +0.000519409 \\
King 5 & $H$ & 1st Feb 1998 & -0.250972 & +0.538105 & -0.260015 & +0.0827334 & -0.00719975 & +0.000205384 \\
King 5 & $K$ & 1st Feb 1998 & -4.03149 & +3.83264 & -1.2277 & +0.211302 & -0.0152649 & +0.000399544 \\
King 5 & $J$ & late 2002 & -1.45036 & +1.67565 & -0.57155 & +0.137235 & -0.0121439 & +0.0003735 \\
King 5 & $H$ & late 2002 & -0.661541 & +0.836397 & -0.314417 & +0.0918192 & -0.00805127 & +0.000233455 \\
King 5 & $K$ & late 2002 & -3.2959 & +3.25895 & -1.08911 & +0.199294 & -0.014921 & +0.000400117 \\
\hline King 30 & $J$ & 1st Feb 1998 & 20.1031 & -16.2944 & +4.68181 & -0.590835 & +0.0366833 & -0.000897527 \\
King 30 & $H$ & 1st Feb 1998 & 6.22703 & -5.3524 & +1.38243 & -0.118854 & +0.00439958 & $-4.84393 \mathrm{E}-05$ \\
King 30 & $K$ & 1st Feb 1998 & 1.10241 & -0.414896 & -0.167382 & +0.0997248 & -0.0101226 & +0.000321986 \\
King 30 & $J$ & late 2002 & 2.68975 & -2.81183 & +0.891735 & -0.0726183 & +0.00206419 & $+6.85053 \mathrm{E}-06$ \\
King 30 & $H$ & late 2002 & 5.27875 & -4.78961 & +1.34219 & -0.125257 & +0.00545339 & $-8.95878 \mathrm{E}-05$ \\
King 30 & $K$ & late 2002 & 1.77318 & -0.96521 & -0.0362266 & +0.0906545 & -0.0101929 & +0.000341137 \\
\hline King 100 & $J$ & 1st Feb 1998 & 13.8405 & -12.4158 & +3.73345 & -0.477173 & +0.0300492 & -0.000745722 \\
King 100 & $H$ & 1st Feb 1998 & 13.2215 & -11.4628 & +3.34063 & -0.412183 & +0.0252202 & -0.000612856 \\
King 100 & $K$ & 1st Feb 1998 & 2.38636 & -1.65819 & +0.25375 & +0.0346631 & -0.00541847 & +0.000194137 \\
King 100 & $J$ & late 2002 & 5.26492 & -5.45479 & +1.84973 & -0.232428 & +0.0146361 & -0.00036889 \\
King 100 & $H$ & late 2002 & 9.96452 & -8.90597 & +2.63648 & -0.31267 & +0.0182118 & -0.000419879 \\
King 100 & $K$ & late 2002 & 3.10281 & -2.08637 & +0.294448 & +0.0458681 & -0.00728688 & +0.000267461 \\
\hline
\end{tabular}


Table 6.16: Fit results of cluster sizes for a "standard" cluster, using various filters available for the NICMOS camera NIC2 $(J=$ F110W, $H=$ F160W, $K=\mathrm{F} 205 \mathrm{~W}$ ) and 2 different epochs of observation ("1st Feb 1998" shows severe blurring due to focus shifts in the pre-cooler phase, which is not present for all pre-service mission observations; "late 2002" is an in-focus observation in the post-cooler phase.). See Section 6.2.9. Size' ( $y$ ).

\begin{tabular}{|c|c|c|c|c|c|c|c|c|}
\hline Profile & filter & $a^{\prime}$ & $b^{\prime}$ & $c^{\prime}$ & $d^{\prime}$ & $e^{\prime}$ & $f^{\prime}$ & \\
\hline EFF 15 & $J$ & 1st Feb 1998 & -12.1574 & +6.33127 & -1.30633 & +0.172817 & -0.0108135 & +0.000256823 \\
EFF 15 & $H$ & 1st Feb 1998 & 5.63435 & -5.13825 & +1.52144 & -0.165009 & +0.00876099 & -0.00018414 \\
EFF 15 & $K$ & 1st Feb 1998 & -0.0194342 & +0.0295552 & -0.0497649 & +0.0497244 & -0.00497063 & +0.000150302 \\
EFF 15 & $J$ & late 2002 & 1.84953 & -2.29393 & +0.92442 & -0.107812 & +0.00628089 & -0.000147625 \\
EFF 15 & $H$ & late 2002 & 3.87171 & -3.75112 & +1.17578 & -0.120374 & +0.00583693 & -0.000108527 \\
EFF 15 & $K$ & late 2002 & 1.36319 & -1.23961 & +0.354356 & -0.00734087 & -0.0011478 & $+5.11466 \mathrm{E}-05$ \\
\hline EFF 25 & $J$ & 1st Feb 1998 & -17.7892 & +10.2271 & -2.19139 & +0.262349 & -0.0150638 & +0.000331967 \\
EFF 25 & $H$ & 1st Feb 1998 & 2.51217 & -2.40888 & +0.792314 & -0.0754728 & +0.00331526 & $-5.36685 \mathrm{E}-05$ \\
EFF 25 & $K$ & 1st Feb 1998 & -1.46588 & +1.10727 & -0.22879 & +0.0565402 & -0.00462692 & +0.000127839 \\
EFF 25 & $J$ & late 2002 & 0.267424 & -0.610077 & +0.43236 & -0.0429438 & +0.00195968 & $-3.36875 \mathrm{E}-05$ \\
EFF 25 & $H$ & late 2002 & 1.50781 & -1.65171 & +0.64128 & -0.0614189 & +0.00275994 & $-4.89501 \mathrm{E}-05$ \\
EFF 25 & $K$ & late 2002 & -0.439494 & +0.235489 & +0.0153897 & +0.0276779 & -0.00305291 & $+9.4622 \mathrm{E}-05$ \\
\hline
\end{tabular}


6.8.2 Parameters of aperture correction fits: Intrinsic sizes 
Table 6.17: Fit result of Eq. (6.7) to different apertures and models, for the correction to infinite aperture, as a function of the intrinsic size of a cluster. PC chip, F555W filter

\begin{tabular}{|c|c|c|c|c|c|c|}
\hline \multicolumn{7}{|c|}{ King 5 } \\
\hline Aperture (pixels) & $a$ & $b$ & $c$ & $d$ & $e$ & $f$ \\
\hline 2 & -0.426038 & -0.136596 & -0.0861804 & +0.0148725 & -0.000989532 & $+2.36101 \mathrm{e}-05$ \\
3 & -0.253541 & +0.0187084 & -0.0942414 & +0.0136279 & -0.000832347 & $+1.88095 \mathrm{e}-05$ \\
4 & -0.213685 & +0.0820324 & -0.0781067 & +0.00952693 & -0.000516199 & $+1.06283 \mathrm{e}-05$ \\
5 & -0.180245 & +0.0768147 & -0.051022 & +0.00475335 & -0.000191085 & $+2.76682 \mathrm{e}-06$ \\
6 & -0.147484 & +0.0510733 & -0.0271736 & +0.00106546 & $+4.37191 \mathrm{e}-05$ & $-2.67164 \mathrm{e}-06$ \\
7 & -0.123417 & +0.0276941 & -0.00998286 & -0.00131093 & +0.00018193 & $-5.63647 \mathrm{e}-06$ \\
8 & -0.105898 & +0.00984873 & +0.00113326 & -0.00264331 & +0.000249236 & $-6.88881 \mathrm{e}-06$ \\
9 & -0.0890763 & -0.00421723 & +0.00824061 & -0.00328716 & +0.000270195 & $-7.03736 \mathrm{e}-06$ \\
10 & -0.0760829 & -0.0112856 & +0.0110047 & -0.00326358 & +0.000248356 & $-6.18611 \mathrm{e}-06$ \\
11 & -0.0651885 & -0.0158698 & +0.0121261 & -0.0030339 & +0.000216003 & $-5.14245 \mathrm{e}-06$ \\
12 & -0.0577756 & -0.0159089 & +0.0111294 & -0.00252785 & +0.000168502 & $-3.7976 \mathrm{e}-06$ \\
13 & -0.050697 & -0.0152317 & +0.00962297 & -0.00199633 & +0.000122222 & $-2.53144 \mathrm{e}-06$ \\
14 & -0.0452285 & -0.0130163 & +0.00764698 & -0.00145699 & $+7.89555 \mathrm{e}-05$ & $-1.40616 \mathrm{e}-06$ \\
15 & -0.0412708 & -0.00938672 & +0.00510787 & -0.000871184 & $+3.44415 \mathrm{e}-05$ & $-2.77989 \mathrm{e}-07$ \\
\hline
\end{tabular}


Table 6.18: Fit result of Eq. (6.7) to different apertures and models, for the correction to infinite aperture, as a function of the intrinsic size of a cluster. PC chip, F555W filter

\begin{tabular}{|c|c|c|c|c|c|c|}
\hline \multicolumn{7}{|c|}{ King 30 } \\
\hline Aperture (pixels) & $a$ & $b$ & $c$ & $d$ & $e$ & $f$ \\
\hline 2 & -0.3835 & -0.765642 & +0.0910683 & -0.00712947 & +0.000301939 & $-5.21251 \mathrm{e}-06$ \\
3 & -0.161718 & -0.523247 & +0.0358429 & -0.000283795 & -0.000113056 & $+4.37674 \mathrm{e}-06$ \\
4 & -0.107487 & -0.337264 & -0.00399272 & +0.00461642 & -0.000411317 & $+1.13031 \mathrm{e}-05$ \\
5 & -0.0865089 & -0.221116 & -0.0254125 & +0.00710234 & -0.000558426 & $+1.46553 \mathrm{e}-05$ \\
6 & -0.0749599 & -0.148568 & -0.0366326 & +0.00829667 & -0.000625769 & $+1.61409 \mathrm{e}-05$ \\
7 & -0.0701872 & -0.0949711 & -0.0437675 & +0.00896644 & -0.000659569 & $+1.68169 \mathrm{e}-05$ \\
8 & -0.0689084 & -0.056331 & -0.0479355 & +0.00925457 & -0.000668674 & $+1.68904 \mathrm{e}-05$ \\
9 & -0.0659416 & -0.0294818 & -0.0491719 & +0.00914777 & -0.000652859 & $+1.63814 \mathrm{e}-05$ \\
10 & -0.0625657 & -0.0108105 & -0.0488939 & +0.00885566 & -0.000625505 & $+1.55969 \mathrm{e}-05$ \\
11 & -0.059248 & +0.00265391 & -0.0477507 & +0.00846553 & -0.000592486 & $+1.46881 \mathrm{e}-05$ \\
12 & -0.0565779 & +0.0133782 & -0.0464083 & +0.0080818 & -0.000561477 & $+1.38592 \mathrm{e}-05$ \\
13 & -0.053317 & +0.0204158 & -0.0445172 & +0.00764742 & -0.000528069 & $+1.29841 \mathrm{e}-05$ \\
14 & -0.0496997 & +0.0246217 & -0.041833 & +0.00708288 & -0.000485161 & $+1.18616 \mathrm{e}-05$ \\
15 & -0.0468754 & +0.0285217 & -0.0398353 & +0.00667399 & -0.000454965 & $+1.10898 \mathrm{e}-05$ \\
\hline
\end{tabular}


Table 6.19: Fit result of Eq. (6.7) to different apertures and models, for the correction to infinite aperture, as a function of the intrinsic size of a cluster. PC chip, F555W filter

\begin{tabular}{|c|c|c|c|c|c|c|}
\hline \multicolumn{7}{|c|}{ EFF 15 } \\
\hline Aperture (pixels) & $a$ & $b$ & $c$ & $d$ & $e$ & $f$ \\
\hline 2 & -0.467985 & -0.488481 & +0.0289793 & -0.000908159 & $-2.1364 \mathrm{e}-08$ & $+5.12344 \mathrm{e}-07$ \\
3 & -0.253351 & -0.307135 & +0.00175459 & +0.00141806 & -0.000105447 & $+2.46419 \mathrm{e}-06$ \\
4 & -0.192055 & -0.19125 & -0.00951212 & +0.00196088 & -0.000115291 & $+2.43585 \mathrm{e}-06$ \\
5 & -0.157092 & -0.134668 & -0.00957336 & +0.00145252 & $-7.61278 \mathrm{e}-05$ & $+1.50643 \mathrm{e}-06$ \\
6 & -0.130444 & -0.106708 & -0.00685717 & +0.000872385 & $-4.04058 \mathrm{e}-05$ & $+7.39037 \mathrm{e}-07$ \\
7 & -0.11322 & -0.0853712 & -0.0052768 & +0.000566775 & $-2.33425 \mathrm{e}-05$ & $+3.96167 \mathrm{e}-07$ \\
8 & -0.101042 & -0.0702356 & -0.00430657 & +0.000419421 & $-1.70926 \mathrm{e}-05$ & $+3.07159 \mathrm{e}-07$ \\
9 & -0.0895293 & -0.0586956 & -0.00355881 & +0.000328037 & $-1.38232 \mathrm{e}-05$ & $+2.66113 \mathrm{e}-07$ \\
10 & -0.0788658 & -0.0509666 & -0.00270373 & +0.000236974 & $-1.07057 \mathrm{e}-05$ & $+2.36366 \mathrm{e}-07$ \\
11 & -0.0700219 & -0.0439451 & -0.00244295 & +0.000235228 & $-1.2713 \mathrm{e}-05$ & $+3.12633 \mathrm{e}-07$ \\
12 & -0.0626445 & -0.0384298 & -0.00190483 & +0.000159835 & $-7.81201 \mathrm{e}-06$ & $+1.80925 \mathrm{e}-07$ \\
13 & -0.0559826 & -0.0335124 & -0.00180523 & +0.000170576 & $-9.55457 \mathrm{e}-06$ & $+2.32684 \mathrm{e}-07$ \\
14 & -0.0498151 & -0.0295678 & -0.00159776 & +0.000161484 & $-9.75509 \mathrm{e}-06$ & $+2.43073 \mathrm{e}-07$ \\
15 & -0.044559 & -0.0262237 & -0.00140946 & +0.000148385 & $-9.6613 \mathrm{e}-06$ & $+2.55557 \mathrm{e}-07$ \\
\hline
\end{tabular}


Table 6.20: Fit result of Eq. (6.7) to different apertures and models, for the correction to infinite aperture, as a function of the intrinsic size of a cluster. WF3 chip, F555W filter

\begin{tabular}{|c|c|c|c|c|c|c|}
\hline \multicolumn{7}{|c|}{ King 5 } \\
\hline Aperture (pixels) & $a$ & $b$ & $c$ & $d$ & $e$ & $f$ \\
\hline 2 & -0.343217 & -0.139042 & -0.0866861 & +0.0149747 & -0.000997889 & $+2.38529 \mathrm{e}-05$ \\
3 & -0.188471 & +0.0272345 & -0.0979988 & +0.014184 & -0.000869693 & $+1.97378 \mathrm{e}-05$ \\
4 & -0.145765 & +0.0814955 & -0.0785946 & +0.00961248 & -0.000523544 & $+1.08528 \mathrm{e}-05$ \\
5 & -0.119422 & +0.0765121 & -0.0512112 & +0.00477206 & -0.000192905 & $+2.83464 \mathrm{e}-06$ \\
6 & -0.0969559 & +0.0527979 & -0.0274306 & +0.00105827 & $+4.46585 \mathrm{e}-05$ & $-2.68042 \mathrm{e}-06$ \\
7 & -0.0760369 & +0.0278805 & -0.00969089 & -0.00139504 & +0.000188023 & $-5.77875 \mathrm{e}-06$ \\
8 & -0.0598124 & +0.00835219 & +0.00180109 & -0.00275704 & +0.000256148 & $-7.03202 \mathrm{e}-06$ \\
9 & -0.0471531 & -0.00522251 & +0.0086179 & -0.00333541 & +0.000271529 & $-7.01923 \mathrm{e}-06$ \\
10 & -0.0389342 & -0.0127713 & +0.0117708 & -0.00337635 & +0.000254044 & $-6.2736 \mathrm{e}-06$ \\
11 & -0.0322645 & -0.0170747 & +0.0127853 & -0.00312398 & +0.000220076 & $-5.19269 \mathrm{e}-06$ \\
12 & -0.0277099 & -0.0175305 & +0.0118183 & -0.00260648 & +0.000171137 & $-3.80217 \mathrm{e}-06$ \\
13 & -0.0237682 & -0.0163978 & +0.0101098 & -0.00203923 & +0.000122517 & $-2.48614 \mathrm{e}-06$ \\
14 & -0.0205814 & -0.014108 & +0.00798676 & -0.00146729 & $+7.67499 \mathrm{e}-05$ & $-1.29518 \mathrm{e}-06$ \\
15 & -0.018043 & -0.0114669 & +0.00590141 & -0.00096043 & $+3.80676 \mathrm{e}-05$ & $-3.20871 \mathrm{e}-07$ \\
\hline
\end{tabular}


Table 6.21: Fit result of Eq. (6.7) to different apertures and models, for the correction to infinite aperture, as a function of the intrinsic size of a cluster. WF3 chip, F555W filter

\begin{tabular}{|c|c|c|c|c|c|c|}
\hline \multicolumn{7}{|c|}{ King 30 } \\
\hline Aperture (pixels) & $a$ & $b$ & $c$ & $d$ & $e$ & $f$ \\
\hline 2 & -0.301938 & -0.769798 & +0.0909698 & -0.00712295 & +0.000302977 & $-5.25793 \mathrm{e}-06$ \\
3 & -0.0947831 & -0.518496 & +0.0330069 & +0.0001133 & -0.000137099 & $+4.92056 \mathrm{e}-06$ \\
4 & -0.0398307 & -0.339737 & -0.00407076 & +0.00459379 & -0.000407134 & $+1.11517 \mathrm{e}-05$ \\
5 & -0.0261104 & -0.222049 & -0.0258731 & +0.00714658 & -0.000559794 & $+1.46668 \mathrm{e}-05$ \\
6 & -0.0234392 & -0.146405 & -0.0378362 & +0.00843162 & -0.000632339 & $+1.62651 \mathrm{e}-05$ \\
7 & -0.0228308 & -0.0933415 & -0.044637 & +0.00904469 & -0.000661937 & $+1.6827 \mathrm{e}-05$ \\
8 & -0.0234847 & -0.0565844 & -0.0483172 & +0.00929001 & -0.000669692 & $+1.68934 \mathrm{e}-05$ \\
9 & -0.0244382 & -0.0293747 & -0.049599 & +0.00917832 & -0.000652608 & $+1.63305 \mathrm{e}-05$ \\
10 & -0.026445 & -0.00885818 & -0.0498729 & +0.00896762 & -0.000630663 & $+1.56775 \mathrm{e}-05$ \\
11 & -0.027269 & +0.00509821 & -0.0488539 & +0.00860091 & -0.000599651 & $+1.48294 \mathrm{e}-05$ \\
12 & -0.027888 & +0.015614 & -0.0473141 & +0.00817894 & -0.000565593 & $+1.39148 \mathrm{e}-05$ \\
13 & -0.0274377 & +0.0225115 & -0.0452125 & +0.00769632 & -0.000527996 & $+1.29164 \mathrm{e}-05$ \\
14 & -0.0266885 & +0.0272389 & -0.0427145 & +0.00716421 & -0.000487547 & $+1.18608 \mathrm{e}-05$ \\
15 & -0.0253624 & +0.029722 & -0.040093 & +0.00665291 & -0.000450163 & $+1.09087 \mathrm{e}-05$ \\
\hline
\end{tabular}


Table 6.22: Fit result of Eq. (6.7) to different apertures and models, for the correction to infinite aperture, as a function of the intrinsic size of a cluster. WF3 chip, F555W filter

\begin{tabular}{|c|c|c|c|c|c|c|}
\hline \multicolumn{7}{|c|}{ EFF 15 } \\
\hline Aperture (pixels) & $a$ & $b$ & $c$ & $d$ & $e$ & $f$ \\
\hline 2 & -0.38549 & -0.493638 & +0.029603 & -0.00100761 & $+6.40292 \mathrm{e}-06$ & $+3.891 \mathrm{e}-07$ \\
3 & -0.186551 & -0.303161 & -0.000318413 & +0.00170018 & -0.00012377 & $+2.93347 \mathrm{e}-06$ \\
4 & -0.124193 & -0.194666 & -0.00875745 & +0.00181114 & -0.000104326 & $+2.17472 \mathrm{e}-06$ \\
5 & -0.095772 & -0.138253 & -0.00840869 & +0.00121683 & $-5.80395 \mathrm{e}-05$ & $+1.04099 \mathrm{e}-06$ \\
6 & -0.0785291 & -0.107328 & -0.00636706 & +0.000716535 & $-2.66562 \mathrm{e}-05$ & $+3.58918 \mathrm{e}-07$ \\
7 & -0.0649638 & -0.0867692 & -0.00459549 & +0.000402047 & $-9.93606 \mathrm{e}-06$ & $+4.03718 \mathrm{e}-08$ \\
8 & -0.0551751 & -0.0722107 & -0.00363422 & +0.000284659 & $-7.07593 \mathrm{e}-06$ & $+5.88729 \mathrm{e}-08$ \\
9 & -0.0472316 & -0.0608484 & -0.00285465 & +0.000197532 & $-4.52905 \mathrm{e}-06$ & $+4.33668 \mathrm{e}-08$ \\
10 & -0.0416458 & -0.0518473 & -0.00233845 & +0.000147243 & $-3.39475 \mathrm{e}-06$ & $+4.377 \mathrm{e}-08$ \\
11 & -0.0371055 & -0.0450427 & -0.00174656 & $+7.82894 \mathrm{e}-05$ & $-1.06734 \mathrm{e}-07$ & $-2.52376 \mathrm{e}-08$ \\
12 & -0.0330528 & -0.0393012 & -0.00141595 & $+5.6084 \mathrm{e}-05$ & $+3.39864 \mathrm{e}-08$ & $-1.56468 \mathrm{e}-08$ \\
13 & -0.0288803 & -0.035054 & -0.000954172 & $+4.38475 \mathrm{e}-06$ & $+2.75389 \mathrm{e}-06$ & $-7.76727 \mathrm{e}-08$ \\
14 & -0.0254902 & -0.0307371 & -0.000810798 & $-2.95168 \mathrm{e}-06$ & $+2.8147 \mathrm{e}-06$ & $-7.84767 \mathrm{e}-08$ \\
15 & -0.0225039 & -0.0270641 & -0.000851373 & $+3.39294 \mathrm{e}-05$ & $-9.68836 \mathrm{e}-07$ & $+3.23473 \mathrm{e}-08$ \\
\hline
\end{tabular}


Table 6.23: Fit result of Eq. (6.7) to different apertures and models, for the correction to infinite aperture, as a function of the intrinsic size of a cluster. ACS/WFC1 chip, F555W filter (chip 2 is equivalent)

\begin{tabular}{|c|c|c|c|c|c|c|}
\hline \multicolumn{7}{|c|}{ King 5 } \\
\hline Aperture (pixels) & $a$ & $b$ & $c$ & $d$ & $e$ & $f$ \\
\hline 2 & -0.398674 & -0.132897 & -0.0874645 & +0.015086 & -0.00100741 & $+2.41505 \mathrm{e}-05$ \\
3 & -0.208959 & +0.017082 & -0.09545 & +0.0139436 & -0.000860482 & $+1.96398 \mathrm{e}-05$ \\
4 & -0.172044 & +0.0820237 & -0.0797896 & +0.0099029 & -0.000547678 & $+1.15244 \mathrm{e}-05$ \\
5 & -0.147693 & +0.0784211 & -0.0523899 & +0.00501035 & -0.000211438 & $+3.33508 \mathrm{e}-06$ \\
6 & -0.125328 & +0.0550134 & -0.028654 & +0.00130393 & $+2.53604 \mathrm{e}-05$ & $-2.15332 \mathrm{e}-06$ \\
7 & -0.106504 & +0.0318753 & -0.0113422 & -0.00109967 & +0.000165877 & $-5.18707 \mathrm{e}-06$ \\
8 & -0.0893515 & +0.0123295 & +0.000139444 & -0.00245895 & +0.000233734 & $-6.43234 \mathrm{e}-06$ \\
9 & -0.0741676 & -0.00270584 & +0.00748727 & -0.00312099 & +0.00025489 & $-6.56425 \mathrm{e}-06$ \\
10 & -0.0629929 & -0.0114838 & +0.0109839 & -0.0032113 & +0.000240733 & $-5.9012 \mathrm{e}-06$ \\
11 & -0.0554195 & -0.0144846 & +0.0114167 & -0.00286322 & +0.000199971 & $-4.64724 \mathrm{e}-06$ \\
12 & -0.0486425 & -0.0161447 & +0.0109681 & -0.0024397 & +0.000158222 & $-3.45102 \mathrm{e}-06$ \\
13 & -0.0449032 & -0.013889 & +0.00881288 & -0.00179763 & +0.000104036 & $-1.98483 \mathrm{e}-06$ \\
14 & -0.0409761 & -0.0118749 & +0.0068691 & -0.00126165 & $+6.12334 \mathrm{e}-05$ & $-8.80661 \mathrm{e}-07$ \\
15 & -0.0383159 & -0.00852972 & +0.00443852 & -0.000691369 & $+1.75978 \mathrm{e}-05$ & $+2.31896 \mathrm{e}-07$ \\
\hline
\end{tabular}


Table 6.24: Fit result of Eq. (6.7) to different apertures and models, for the correction to infinite aperture, as a function of the intrinsic size of a cluster. ACS/WFC1 chip, F555W filter (chip 2 is equivalent)

\begin{tabular}{|c|c|c|c|c|c|c|}
\hline \multicolumn{7}{|c|}{ King 30 } \\
\hline Aperture (pixels) & $a$ & $b$ & $c$ & $d$ & $e$ & $f$ \\
\hline 2 & -0.355942 & -0.760461 & +0.089143 & -0.00687739 & +0.000286982 & $-4.87563 \mathrm{e}-06$ \\
3 & -0.118651 & -0.527209 & +0.0361609 & -0.000318572 & -0.000109747 & $+4.26438 \mathrm{e}-06$ \\
4 & -0.0657887 & -0.342186 & -0.00308969 & +0.00446671 & -0.000398934 & $+1.09424 \mathrm{e}-05$ \\
5 & -0.0530972 & -0.223214 & -0.0250121 & +0.00699647 & -0.000547895 & $+1.43258 \mathrm{e}-05$ \\
6 & -0.0505818 & -0.146978 & -0.0371755 & +0.00831202 & -0.00062245 & $+1.59702 \mathrm{e}-05$ \\
7 & -0.0514446 & -0.0919757 & -0.0445655 & +0.00900466 & -0.000656951 & $+1.6644 \mathrm{e}-05$ \\
8 & -0.0514832 & -0.0545999 & -0.0484293 & +0.00926896 & -0.00066556 & $+1.67254 \mathrm{e}-05$ \\
9 & -0.0507511 & -0.0281879 & -0.0495274 & +0.00914747 & -0.000648976 & $+1.62 \mathrm{e}-05$ \\
10 & -0.0498153 & -0.00930802 & -0.0492446 & +0.00884519 & -0.000620308 & $+1.5369 \mathrm{e}-05$ \\
11 & -0.0491256 & +0.00472795 & -0.048382 & +0.00851298 & -0.000592089 & $+1.45968 \mathrm{e}-05$ \\
12 & -0.0479808 & +0.0144529 & -0.0465673 & +0.00804938 & -0.000555299 & $+1.3617 \mathrm{e}-05$ \\
13 & -0.0471617 & +0.0221165 & -0.0448252 & +0.00763215 & -0.000522917 & $+1.2769 \mathrm{e}-05$ \\
14 & -0.0455847 & +0.026513 & -0.0422449 & +0.00709523 & -0.000482514 & $+1.17191 \mathrm{e}-05$ \\
15 & -0.0441257 & +0.0296988 & -0.0397833 & +0.00659238 & -0.000444824 & $+1.07422 \mathrm{e}-05$ \\
\hline
\end{tabular}


Table 6.25: Fit result of Eq. (6.7) to different apertures and models, for the correction to infinite aperture, as a function of the intrinsic size of a cluster. ACS/WFC1 chip, F555W filter (chip 2 is equivalent)

\begin{tabular}{|c|c|c|c|c|c|c|}
\hline \multicolumn{7}{|c|}{ EFF 15 } \\
\hline Aperture (pixels) & $a$ & $b$ & $c$ & $d$ & $e$ & $f$ \\
\hline 2 & -0.438427 & -0.489241 & +0.0302078 & -0.00122199 & $+2.59247 \mathrm{e}-05$ & $-1.83598 \mathrm{e}-07$ \\
3 & -0.209021 & -0.312719 & +0.00328145 & +0.00114218 & $-8.44961 \mathrm{e}-05$ & $+1.92025 \mathrm{e}-06$ \\
4 & -0.150322 & -0.195902 & -0.00819165 & +0.0017053 & $-9.5199 \mathrm{e}-05$ & $+1.90367 \mathrm{e}-06$ \\
5 & -0.123678 & -0.137212 & -0.00842157 & +0.00117856 & $-5.27575 \mathrm{e}-05$ & $+8.55848 \mathrm{e}-07$ \\
6 & -0.106465 & -0.106297 & -0.00617475 & +0.000632557 & $-1.78492 \mathrm{e}-05$ & $+8.124 \mathrm{e}-08$ \\
7 & -0.0935284 & -0.0854713 & -0.00433556 & +0.000297178 & $+6.26081 \mathrm{e}-07$ & $-2.85663 \mathrm{e}-07$ \\
8 & -0.0826082 & -0.0717049 & -0.00294076 & $+9.87344 \mathrm{e}-05$ & $+9.58297 \mathrm{e}-06$ & $-4.26449 \mathrm{e}-07$ \\
9 & -0.0731633 & -0.0604325 & -0.00219101 & $+1.36612 \mathrm{e}-05$ & $+1.24553 \mathrm{e}-05$ & $-4.63917 \mathrm{e}-07$ \\
10 & -0.0652346 & -0.0524734 & -0.0013251 & $-9.0109 \mathrm{e}-05$ & $+1.71436 \mathrm{e}-05$ & $-5.47037 \mathrm{e}-07$ \\
11 & -0.0586628 & -0.0458916 & -0.000774047 & -0.000146189 & $+1.9362 \mathrm{e}-05$ & $-5.8822 \mathrm{e}-07$ \\
12 & -0.0531572 & -0.0400005 & -0.000571944 & -0.000145613 & $+1.78462 \mathrm{e}-05$ & $-5.35236 \mathrm{e}-07$ \\
13 & -0.0489136 & -0.0347115 & -0.000543306 & -0.000126437 & $+1.55462 \mathrm{e}-05$ & $-4.68505 \mathrm{e}-07$ \\
14 & -0.0447563 & -0.0304532 & -0.000456213 & -0.000113559 & $+1.35677 \mathrm{e}-05$ & $-4.05904 \mathrm{e}-07$ \\
15 & -0.0411696 & -0.0273396 & -0.000155292 & -0.000145799 & $+1.53069 \mathrm{e}-05$ & $-4.46165 \mathrm{e}-07$ \\
\hline
\end{tabular}


Table 6.26: Fit result of Eq. (6.7) to different apertures and models, for the correction to infinite aperture, as a function of the intrinsic size of a cluster. NICMOS/NIC2 chip, F110W filter, pre-cooler

\begin{tabular}{|c|c|c|c|c|c|c|}
\hline \multicolumn{7}{|c|}{ King 5 } \\
\hline Aperture (pixels) & $a$ & $b$ & $c$ & $d$ & $e$ & $f$ \\
\hline 2 & -0.800688 & -0.130052 & -0.0673997 & +0.0113793 & -0.000747284 & $+1.77132 \mathrm{e}-05$ \\
3 & -0.484616 & +0.00891831 & -0.0824723 & +0.0118641 & -0.000725057 & $+1.64406 \mathrm{e}-05$ \\
4 & -0.302567 & +0.0415784 & -0.065855 & +0.00820699 & -0.00045322 & $+9.5225 \mathrm{e}-06$ \\
5 & -0.214979 & +0.0505999 & -0.0475831 & +0.00482632 & -0.00021948 & $+3.82738 \mathrm{e}-06$ \\
6 & -0.170281 & +0.0425738 & -0.0296033 & +0.00187301 & $-2.65806 \mathrm{e}-05$ & $-7.02804 \mathrm{e}-07$ \\
7 & -0.140987 & +0.0284744 & -0.0145264 & -0.000342716 & +0.000107954 & $-3.69675 \mathrm{e}-06$ \\
8 & -0.119386 & +0.0134033 & -0.00323463 & -0.00182108 & +0.000189797 & $-5.37803 \mathrm{e}-06$ \\
9 & -0.101079 & +0.000136667 & +0.00467726 & -0.0026729 & +0.00022749 & $-5.9697 \mathrm{e}-06$ \\
10 & -0.0859178 & -0.00920758 & +0.00906872 & -0.00294344 & +0.000226447 & $-5.63424 \mathrm{e}-06$ \\
11 & -0.0742331 & -0.0133775 & +0.010435 & -0.00277322 & +0.000198692 & $-4.71104 \mathrm{e}-06$ \\
12 & -0.0655578 & -0.0139692 & +0.00995018 & -0.00236344 & +0.000158207 & $-3.54562 \mathrm{e}-06$ \\
13 & -0.0571691 & -0.0142732 & +0.00903297 & -0.00192523 & +0.000117873 & $-2.41657 \mathrm{e}-06$ \\
14 & -0.0503899 & -0.0130529 & +0.00748916 & -0.00144235 & $+7.75503 \mathrm{e}-05$ & $-1.34264 \mathrm{e}-06$ \\
15 & -0.0464203 & -0.00950285 & +0.00524322 & -0.00091905 & $+3.7914 \mathrm{e}-05$ & $-3.46032 \mathrm{e}-07$ \\
\hline
\end{tabular}


Table 6.27: Fit result of Eq. (6.7) to different apertures and models, for the correction to infinite aperture, as a function of the intrinsic size of a cluster. NICMOS/NIC2 chip, F110W filter, pre-cooler

\begin{tabular}{|c|c|c|c|c|c|c|}
\hline \multicolumn{7}{|c|}{ King 30 } \\
\hline Aperture (pixels) & $a$ & $b$ & $c$ & $d$ & $e$ & $f$ \\
\hline 2 & -0.760738 & -0.689552 & +0.0806142 & -0.00613326 & +0.000247703 & $-3.99816 \mathrm{e}-06$ \\
3 & -0.40033 & -0.490233 & +0.032309 & $+4.66148 \mathrm{e}-05$ & -0.000132192 & $+4.83143 \mathrm{e}-06$ \\
4 & -0.213607 & -0.359133 & +0.00577086 & +0.00319433 & -0.000318897 & $+9.08143 \mathrm{e}-06$ \\
5 & -0.131945 & -0.252195 & -0.0151787 & +0.00569048 & -0.000468351 & $+1.25135 \mathrm{e}-05$ \\
6 & -0.0997125 & -0.173551 & -0.0290165 & +0.00724365 & -0.000557584 & $+1.44968 \mathrm{e}-05$ \\
7 & -0.0860145 & -0.112785 & -0.0385615 & +0.00823028 & -0.000610501 & $+1.56063 \mathrm{e}-05$ \\
8 & -0.0791829 & -0.0707277 & -0.0438508 & +0.00867467 & -0.000629775 & $+1.59252 \mathrm{e}-05$ \\
9 & -0.0743961 & -0.039737 & -0.0464033 & +0.00875378 & -0.000626046 & $+1.57056 \mathrm{e}-05$ \\
10 & -0.0701757 & -0.0180948 & -0.0469981 & +0.00858359 & -0.000606826 & $+1.51256 \mathrm{e}-05$ \\
11 & -0.0659551 & -0.00325242 & -0.0461585 & +0.00822576 & -0.000575398 & $+1.42434 \mathrm{e}-05$ \\
12 & -0.0621904 & +0.0087244 & -0.0452712 & +0.00791667 & -0.000549751 & $+1.35517 \mathrm{e}-05$ \\
13 & -0.0578679 & +0.0157329 & -0.0430674 & +0.00740645 & -0.000510064 & $+1.25047 \mathrm{e}-05$ \\
14 & -0.0542968 & +0.0221954 & -0.0413427 & +0.00700572 & -0.000478962 & $+1.16832 \mathrm{e}-05$ \\
15 & -0.050878 & +0.0258332 & -0.0389878 & +0.00651984 & -0.000442637 & $+1.07451 \mathrm{e}-05$ \\
\hline
\end{tabular}


Table 6.28: Fit result of Eq. (6.7) to different apertures and models, for the correction to infinite aperture, as a function of the intrinsic size of a cluster. NICMOS/NIC2 chip, F110W filter, pre-cooler

\begin{tabular}{|c|c|c|c|c|c|c|}
\hline \multicolumn{7}{|c|}{ EFF 15 } \\
\hline Aperture (pixels) & $a$ & $b$ & $c$ & $d$ & $e$ & $f$ \\
\hline 2 & -0.838525 & -0.452545 & +0.0340376 & -0.00220815 & $+9.48796 \mathrm{e}-05$ & $-1.82494 \mathrm{e}-06$ \\
3 & -0.486445 & -0.295696 & +0.00516053 & +0.000858102 & $-7.29587 \mathrm{e}-05$ & $+1.79567 \mathrm{e}-06$ \\
4 & -0.292553 & -0.21783 & -0.000369534 & +0.000860213 & $-5.40794 \mathrm{e}-05$ & $+1.13561 \mathrm{e}-06$ \\
5 & -0.200058 & -0.160667 & -0.00335156 & +0.000804905 & $-4.21794 \mathrm{e}-05$ & $+7.93677 \mathrm{e}-07$ \\
6 & -0.15654 & -0.121745 & -0.00440746 & +0.000692361 & $-3.28266 \mathrm{e}-05$ & $+5.9041 \mathrm{e}-07$ \\
7 & -0.130881 & -0.0944734 & -0.00420458 & +0.000495367 & $-1.90595 \mathrm{e}-05$ & $+2.69588 \mathrm{e}-07$ \\
8 & -0.113676 & -0.0767345 & -0.00360473 & +0.000360995 & $-1.28103 \mathrm{e}-05$ & $+1.79912 \mathrm{e}-07$ \\
9 & -0.100062 & -0.0631468 & -0.00305299 & +0.000263473 & $-8.34613 \mathrm{e}-06$ & $+1.05623 \mathrm{e}-07$ \\
10 & -0.0880728 & -0.0546453 & -0.00201525 & +0.000117005 & $-6.64403 \mathrm{e}-07$ & $-4.93456 \mathrm{e}-08$ \\
11 & -0.0785702 & -0.0463333 & -0.00194654 & +0.000125373 & $-2.55596 \mathrm{e}-06$ & $+7.62772 \mathrm{e}-09$ \\
12 & -0.0694648 & -0.0409303 & -0.0013735 & $+6.58678 \mathrm{e}-05$ & $-5.24244 \mathrm{e}-07$ & $-8.51217 \mathrm{e}-09$ \\
13 & -0.0619132 & -0.0359096 & -0.00111823 & $+3.96193 \mathrm{e}-05$ & $+8.65713 \mathrm{e}-07$ & $-4.94977 \mathrm{e}-08$ \\
14 & -0.0553778 & -0.0313544 & -0.00102871 & $+4.6912 \mathrm{e}-05$ & $-6.41072 \mathrm{e}-07$ & $+1.28284 \mathrm{e}-09$ \\
15 & -0.0495667 & -0.0276055 & -0.000981324 & $+5.65395 \mathrm{e}-05$ & $-1.83564 \mathrm{e}-06$ & $+3.35088 \mathrm{e}-08$ \\
\hline
\end{tabular}


Table 6.29: Fit result of Eq. (6.7) to different apertures and models, for the correction to infinite aperture, as a function of the intrinsic size of a cluster. NICMOS/NIC2 chip, F110W filter, post-cooler

\begin{tabular}{|c|c|c|c|c|c|c|}
\hline \multicolumn{7}{|c|}{ King 5 } \\
\hline Aperture (pixels) & $a$ & $b$ & $c$ & $d$ & $e$ & $f$ \\
\hline 2 & -0.454081 & -0.144799 & -0.0821082 & +0.0142782 & -0.000953572 & $+2.28335 \mathrm{e}-05$ \\
3 & -0.286568 & +0.0254188 & -0.0961705 & +0.0139593 & -0.000857867 & $+1.9508 \mathrm{e}-05$ \\
4 & -0.22107 & +0.0733592 & -0.0760525 & +0.00936262 & -0.000512612 & $+1.06789 \mathrm{e}-05$ \\
5 & -0.195111 & +0.0766789 & -0.0513485 & +0.00487541 & -0.000203336 & $+3.14854 \mathrm{e}-06$ \\
6 & -0.164963 & +0.0537226 & -0.0280591 & +0.00123319 & $+2.98709 \mathrm{e}-05$ & $-2.27123 \mathrm{e}-06$ \\
7 & -0.134684 & +0.0293373 & -0.0109773 & -0.00109881 & +0.000164118 & $-5.12512 \mathrm{e}-06$ \\
8 & -0.11056 & +0.00990864 & +0.000394003 & -0.00246326 & +0.000233797 & $-6.44588 \mathrm{e}-06$ \\
9 & -0.0919823 & -0.0032223 & +0.00708724 & -0.0030427 & +0.000250291 & $-6.4787 \mathrm{e}-06$ \\
10 & -0.078301 & -0.0117348 & +0.0107702 & -0.0031897 & +0.000241072 & $-5.95665 \mathrm{e}-06$ \\
11 & -0.0674537 & -0.0147972 & +0.0113211 & -0.00287647 & +0.000203447 & $-4.7925 \mathrm{e}-06$ \\
12 & -0.058821 & -0.0152813 & +0.0103891 & -0.00236665 & +0.000154746 & $-3.39515 \mathrm{e}-06$ \\
13 & -0.0519002 & -0.0145897 & +0.0090703 & -0.0018824 & +0.000112768 & $-2.26256 \mathrm{e}-06$ \\
14 & -0.0463912 & -0.012074 & +0.00692337 & -0.00130705 & $+6.65271 \mathrm{e}-05$ & $-1.05288 \mathrm{e}-06$ \\
15 & -0.0423207 & -0.00898458 & +0.00477376 & -0.000796459 & $+2.76293 \mathrm{e}-05$ & $-6.98929 \mathrm{e}-08$ \\
\hline
\end{tabular}


Table 6.30: Fit result of Eq. (6.7) to different apertures and models, for the correction to infinite aperture, as a function of the intrinsic size of a cluster. NICMOS/NIC2 chip, F110W filter, post-cooler

\begin{tabular}{|c|c|c|c|c|c|c|}
\hline \multicolumn{7}{|c|}{ King 30 } \\
\hline Aperture (pixels) & $a$ & $b$ & $c$ & $d$ & $e$ & $f$ \\
\hline 2 & -0.416109 & -0.764226 & +0.0917339 & -0.00727601 & +0.000312957 & $-5.49128 \mathrm{e}-06$ \\
3 & -0.194339 & -0.513712 & +0.0331371 & $+8.68036 \mathrm{e}-05$ & -0.000136077 & $+4.90796 \mathrm{e}-06$ \\
4 & -0.118883 & -0.343688 & -0.00161921 & +0.0042493 & -0.000385647 & $+1.06494 \mathrm{e}-05$ \\
5 & -0.102512 & -0.220293 & -0.025392 & +0.0070608 & -0.000553198 & $+1.44776 \mathrm{e}-05$ \\
6 & -0.0918257 & -0.145911 & -0.0369518 & +0.0082742 & -0.00062053 & $+1.59439 \mathrm{e}-05$ \\
7 & -0.0812134 & -0.0948419 & -0.0433687 & +0.00883911 & -0.000647045 & $+1.64297 \mathrm{e}-05$ \\
8 & -0.0736232 & -0.0598587 & -0.046529 & +0.00900238 & -0.000649153 & $+1.6358 \mathrm{e}-05$ \\
9 & -0.0687367 & -0.0321584 & -0.0481723 & +0.0089542 & -0.00063674 & $+1.59166 \mathrm{e}-05$ \\
10 & -0.0648555 & -0.0128278 & -0.0482033 & +0.00872333 & -0.000614484 & $+1.52804 \mathrm{e}-05$ \\
11 & -0.0612514 & +0.00142588 & -0.0473808 & +0.00838342 & -0.000584875 & $+1.44546 \mathrm{e}-05$ \\
12 & -0.057717 & +0.0120192 & -0.045973 & +0.00798426 & -0.000552342 & $+1.35749 \mathrm{e}-05$ \\
13 & -0.0543018 & +0.018521 & -0.0436988 & +0.00747684 & -0.000513593 & $+1.25668 \mathrm{e}-05$ \\
14 & -0.0508119 & +0.023858 & -0.0416046 & +0.00702552 & -0.00047945 & $+1.16782 \mathrm{e}-05$ \\
15 & -0.0482084 & +0.0282317 & -0.0396684 & +0.0066155 & -0.000448856 & $+1.0895 \mathrm{e}-05$ \\
\hline
\end{tabular}


Table 6.31: Fit result of Eq. (6.7) to different apertures and models, for the correction to infinite aperture, as a function of the intrinsic size of a cluster. NICMOS/NIC2 chip, F110W filter, post-cooler

\begin{tabular}{|c|c|c|c|c|c|c|}
\hline \multicolumn{7}{|c|}{ EFF 15 } \\
\hline Aperture (pixels) & $a$ & $b$ & $c$ & $d$ & $e$ & $f$ \\
\hline 2 & -0.4975 & -0.493703 & +0.0322144 & -0.00145059 & $+3.74499 \mathrm{e}-05$ & $-4.03483 \mathrm{e}-07$ \\
3 & -0.28525 & -0.30112 & +0.000483293 & +0.00157081 & -0.000114677 & $+2.69004 \mathrm{e}-06$ \\
4 & -0.202357 & -0.198367 & -0.00726074 & +0.00166417 & $-9.76092 \mathrm{e}-05$ & $+2.05176 \mathrm{e}-06$ \\
5 & -0.172156 & -0.135695 & -0.00896615 & +0.00133957 & $-6.75383 \mathrm{e}-05$ & $+1.28612 \mathrm{e}-06$ \\
6 & -0.147384 & -0.104985 & -0.00681161 & +0.0007977 & $-3.20601 \mathrm{e}-05$ & $+4.79318 \mathrm{e}-07$ \\
7 & -0.123463 & -0.0863787 & -0.00467084 & +0.000439843 & $-1.29993 \mathrm{e}-05$ & $+1.1459 \mathrm{e}-07$ \\
8 & -0.105638 & -0.0729173 & -0.00330641 & +0.000232592 & $-2.27483 \mathrm{e}-06$ & $-9.66059 \mathrm{e}-08$ \\
9 & -0.0918005 & -0.0616896 & -0.00251446 & +0.000140932 & $+5.19467 \mathrm{e}-07$ & $-1.14158 \mathrm{e}-07$ \\
10 & -0.081108 & -0.0524107 & -0.00222098 & +0.000134329 & $-1.89814 \mathrm{e}-06$ & $-1.43376 \mathrm{e}-08$ \\
11 & -0.0717877 & -0.0458984 & -0.00150302 & $+3.64255 \mathrm{e}-05$ & $+3.66169 \mathrm{e}-06$ & $-1.40448 \mathrm{e}-07$ \\
12 & -0.0639979 & -0.0394076 & -0.00157726 & $+8.32373 \mathrm{e}-05$ & $-1.07752 \mathrm{e}-06$ & $-9.3906 \mathrm{e}-09$ \\
13 & -0.0567965 & -0.035151 & -0.00108886 & $+2.38069 \mathrm{e}-05$ & $+2.22429 \mathrm{e}-06$ & $-8.49053 \mathrm{e}-08$ \\
14 & -0.0508278 & -0.0308355 & -0.0010148 & $+3.87226 \mathrm{e}-05$ & $+1.09472 \mathrm{e}-07$ & $-2.01306 \mathrm{e}-08$ \\
15 & -0.0460727 & -0.0269755 & -0.000962471 & $+4.13592 \mathrm{e}-05$ & $-3.79949 \mathrm{e}-07$ & $-7.35858 \mathrm{e}-09$ \\
\hline
\end{tabular}


Table 6.32: Fit result of Eq. (6.7) to different apertures and models, for the correction to infinite aperture, as a function of the intrinsic size of a given cluster. NICMOS/NIC2 chip, F160W filter, pre-cooler

\begin{tabular}{|c|c|c|c|c|c|c|}
\hline \multicolumn{7}{|c|}{ King 5 } \\
\hline Aperture (pixels) & $a$ & $b$ & $c$ & $d$ & $e$ & $f$ \\
\hline 2 & -0.92289 & -0.11592 & -0.069983 & +0.0117466 & -0.000771 & $+1.82576 \mathrm{e}-05$ \\
3 & -0.608266 & +0.017602 & -0.0815352 & +0.0115904 & -0.000702527 & $+1.58198 \mathrm{e}-05$ \\
4 & -0.406736 & +0.0466014 & -0.0647431 & +0.00796071 & -0.000434214 & $+9.01095 \mathrm{e}-06$ \\
5 & -0.278116 & +0.0420945 & -0.0444724 & +0.00444174 & -0.000196631 & $+3.29797 \mathrm{e}-06$ \\
6 & -0.216851 & +0.0381296 & -0.029224 & +0.00194271 & $-3.47224 \mathrm{e}-05$ & $-4.74802 \mathrm{e}-07$ \\
7 & -0.17761 & +0.0263733 & -0.0150189 & -0.000188277 & $+9.68101 \mathrm{e}-05$ & $-3.44481 \mathrm{e}-06$ \\
8 & -0.150851 & +0.0138307 & -0.00460578 & -0.00155211 & +0.00017163 & $-4.9597 \mathrm{e}-06$ \\
9 & -0.127763 & +0.0013109 & +0.00313026 & -0.00239558 & +0.000209498 & $-5.56652 \mathrm{e}-06$ \\
10 & -0.10986 & -0.00677987 & +0.00734011 & -0.00267313 & +0.000210438 & $-5.30539 \mathrm{e}-06$ \\
11 & -0.0958513 & -0.0120804 & +0.00942756 & -0.00263766 & +0.000192621 & $-4.63884 \mathrm{e}-06$ \\
12 & -0.0860429 & -0.0135132 & +0.00959104 & -0.00234271 & +0.000159839 & $-3.65123 \mathrm{e}-06$ \\
13 & -0.0778235 & -0.0134738 & +0.00880418 & -0.00194549 & +0.000123311 & $-2.63753 \mathrm{e}-06$ \\
14 & -0.0712835 & -0.0116265 & +0.00723824 & -0.00147168 & $+8.39695 \mathrm{e}-05$ & $-1.5931 \mathrm{e}-06$ \\
15 & -0.0646233 & -0.0101318 & +0.00572666 & -0.00105133 & $+5.06585 \mathrm{e}-05$ & $-7.39961 \mathrm{e}-07$ \\
\hline
\end{tabular}


Table 6.33: Fit result of Eq. (6.7) to different apertures and models, for the correction to infinite aperture, as a function of the intrinsic size of a given cluster. NICMOS/NIC2 chip, F160W filter, pre-cooler

\begin{tabular}{|c|c|c|c|c|c|c|}
\hline \multicolumn{7}{|c|}{ King 30 } \\
\hline Aperture (pixels) & $a$ & $b$ & $c$ & $d$ & $e$ & $f$ \\
\hline 2 & -0.879448 & -0.670498 & +0.0767106 & -0.00562918 & +0.000217404 & $-3.33674 \mathrm{e}-06$ \\
3 & -0.522987 & -0.467635 & +0.028051 & +0.000527101 & -0.000157907 & $+5.33373 \mathrm{e}-06$ \\
4 & -0.317949 & -0.34164 & +0.00256225 & +0.00355414 & -0.000338181 & $+9.45981 \mathrm{e}-06$ \\
5 & -0.199971 & -0.25354 & -0.0131751 & +0.00533976 & -0.000442411 & $+1.18169 \mathrm{e}-05$ \\
6 & -0.149332 & -0.177719 & -0.0269542 & +0.00692388 & -0.000535018 & $+1.39053 \mathrm{e}-05$ \\
7 & -0.12442 & -0.117781 & -0.0367133 & +0.00796949 & -0.000593024 & $+1.51587 \mathrm{e}-05$ \\
8 & -0.110982 & -0.0752806 & -0.0422646 & +0.00845001 & -0.000614699 & $+1.55412 \mathrm{e}-05$ \\
9 & -0.100671 & -0.0442532 & -0.0448759 & +0.00852393 & -0.000609633 & $+1.52676 \mathrm{e}-05$ \\
10 & -0.0931428 & -0.0216553 & -0.0459086 & +0.00842419 & -0.00059525 & $+1.4806 \mathrm{e}-05$ \\
11 & -0.0867837 & -0.00595049 & -0.0454196 & +0.00812862 & -0.000568849 & $+1.40689 \mathrm{e}-05$ \\
12 & -0.0824191 & +0.00644834 & -0.0444318 & +0.00777407 & -0.000538425 & $+1.32236 \mathrm{e}-05$ \\
13 & -0.0783323 & +0.0154737 & -0.0429958 & +0.00739134 & -0.000508068 & $+1.2422 \mathrm{e}-05$ \\
14 & -0.0741561 & +0.0218155 & -0.0411041 & +0.00696504 & -0.000475754 & $+1.15875 \mathrm{e}-05$ \\
15 & -0.0695502 & +0.0251518 & -0.0385582 & +0.00643905 & -0.000436084 & $+1.05544 \mathrm{e}-05$ \\
\hline
\end{tabular}


Table 6.34: Fit result of Eq. (6.7) to different apertures and models, for the correction to infinite aperture, as a function of the intrinsic size of a given cluster. NICMOS/NIC2 chip, F160W filter, pre-cooler

\begin{tabular}{|c|c|c|c|c|c|c|}
\hline \multicolumn{7}{|c|}{ EFF 15 } \\
\hline Aperture (pixels) & $a$ & $b$ & $c$ & $d$ & $e$ & $f$ \\
\hline 2 & -0.957322 & -0.437008 & +0.0308318 & -0.00173596 & $+6.26574 \mathrm{e}-05$ & $-1.02447 \mathrm{e}-06$ \\
3 & -0.606914 & -0.281847 & +0.00373215 & +0.00097559 & $-7.86577 \mathrm{e}-05$ & $+1.91484 \mathrm{e}-06$ \\
4 & -0.394439 & -0.208272 & -0.00121243 & +0.000938516 & $-5.9176 \mathrm{e}-05$ & $+1.27854 \mathrm{e}-06$ \\
5 & -0.265382 & -0.16504 & -0.00118108 & +0.000524758 & $-2.61409 \mathrm{e}-05$ & $+4.56932 \mathrm{e}-07$ \\
6 & -0.203831 & -0.126894 & -0.00279847 & +0.000506719 & $-2.24222 \mathrm{e}-05$ & $+3.68364 \mathrm{e}-07$ \\
7 & -0.167601 & -0.0991213 & -0.00309737 & +0.000390519 & $-1.432 \mathrm{e}-05$ & $+1.93781 \mathrm{e}-07$ \\
8 & -0.144374 & -0.0805901 & -0.00262643 & +0.000239703 & $-5.22206 \mathrm{e}-06$ & $+4.55103 \mathrm{e}-09$ \\
9 & -0.12583 & -0.0662386 & -0.00248815 & +0.000215821 & $-6.3552 \mathrm{e}-06$ & $+7.96266 \mathrm{e}-08$ \\
10 & -0.110454 & -0.056622 & -0.00183369 & +0.000113902 & $-8.88243 \mathrm{e}-07$ & $-3.70472 \mathrm{e}-08$ \\
11 & -0.0989395 & -0.0485307 & -0.00154893 & $+8.70928 \mathrm{e}-05$ & $-8.00567 \mathrm{e}-07$ & $-1.47172 \mathrm{e}-08$ \\
12 & -0.08962 & -0.0419063 & -0.00114939 & $+2.26635 \mathrm{e}-05$ & $+3.37745 \mathrm{e}-06$ & $-1.24782 \mathrm{e}-07$ \\
13 & -0.0817135 & -0.0363783 & -0.00105229 & $+3.2023 \mathrm{e}-05$ & $+1.2644 \mathrm{e}-06$ & $-5.01439 \mathrm{e}-08$ \\
14 & -0.074622 & -0.0316489 & -0.000914176 & $+2.51952 \mathrm{e}-05$ & $+1.03214 \mathrm{e}-06$ & $-4.00609 \mathrm{e}-08$ \\
15 & -0.067958 & -0.0281055 & -0.000670926 & $-4.86094 \mathrm{e}-06$ & $+2.84041 \mathrm{e}-06$ & $-8.44092 \mathrm{e}-08$ \\
\hline
\end{tabular}


Table 6.35: Fit result of Eq. (6.7) to different apertures and models, for the correction to infinite aperture, as a function of the intrinsic size of a given cluster. NICMOS/NIC2 chip, F160W filter, post-cooler

\begin{tabular}{|c|c|c|c|c|c|c|}
\hline \multicolumn{7}{|c|}{ King 5 } \\
\hline Aperture (pixels) & $a$ & $b$ & $c$ & $d$ & $e$ & $f$ \\
\hline 2 & -0.697675 & -0.117826 & -0.0798897 & +0.0135064 & -0.000891567 & $+2.12036 \mathrm{e}-05$ \\
3 & -0.458122 & +0.0325701 & -0.0915974 & +0.0130496 & -0.000792517 & $+1.78702 \mathrm{e}-05$ \\
4 & -0.289569 & +0.0486726 & -0.0677942 & +0.0083315 & -0.000453201 & $+9.38141 \mathrm{e}-06$ \\
5 & -0.213436 & +0.0508897 & -0.0466837 & +0.00455515 & -0.000195042 & $+3.12421 \mathrm{e}-06$ \\
6 & -0.189971 & +0.047348 & -0.0292699 & +0.00166052 & $-6.00333 \mathrm{e}-06$ & $-1.30334 \mathrm{e}-06$ \\
7 & -0.170576 & +0.0314869 & -0.0129619 & -0.000757935 & +0.000142362 & $-4.6371 \mathrm{e}-06$ \\
8 & -0.150358 & +0.0154644 & -0.00186136 & -0.00214247 & +0.000215015 & $-6.04542 \mathrm{e}-06$ \\
9 & -0.124714 & -0.0011969 & +0.00634119 & -0.00297785 & +0.000249809 & $-6.54643 \mathrm{e}-06$ \\
10 & -0.102401 & -0.0117737 & +0.0103084 & -0.00313286 & +0.000239407 & $-5.96061 \mathrm{e}-06$ \\
11 & -0.0878762 & -0.0152333 & +0.0111084 & -0.00286955 & +0.000205594 & $-4.89695 \mathrm{e}-06$ \\
12 & -0.0783095 & -0.0164168 & +0.0108949 & -0.00250444 & +0.000167958 & $-3.79324 \mathrm{e}-06$ \\
13 & -0.072562 & -0.0140948 & +0.00927589 & -0.00199098 & +0.000124293 & $-2.61718 \mathrm{e}-06$ \\
14 & -0.0661908 & -0.0117099 & +0.00735753 & -0.00146245 & $+8.16384 \mathrm{e}-05$ & $-1.50236 \mathrm{e}-06$ \\
15 & -0.0598453 & -0.00924152 & +0.00536451 & -0.000961582 & $+4.24424 \mathrm{e}-05$ & $-4.91927 \mathrm{e}-07$ \\
\hline
\end{tabular}


Table 6.36: Fit result of Eq. (6.7) to different apertures and models, for the correction to infinite aperture, as a function of the intrinsic size of a given cluster. NICMOS/NIC2 chip, F160W filter, post-cooler

\begin{tabular}{|c|c|c|c|c|c|c|}
\hline \multicolumn{7}{|c|}{ King 30 } \\
\hline Aperture (pixels) & $a$ & $b$ & $c$ & $d$ & $e$ & $f$ \\
\hline 2 & -0.651874 & -0.70926 & +0.0812979 & -0.00602911 & +0.000237688 & $-3.75496 \mathrm{e}-06$ \\
3 & -0.365023 & -0.480755 & +0.0270868 & +0.000816176 & -0.000180336 & $+5.92949 \mathrm{e}-06$ \\
4 & -0.197824 & -0.355255 & +0.00384598 & +0.00344926 & -0.000333512 & $+9.38928 \mathrm{e}-06$ \\
5 & -0.130475 & -0.249527 & -0.0160613 & +0.00578301 & -0.000472175 & $+1.25551 \mathrm{e}-05$ \\
6 & -0.118445 & -0.162146 & -0.0322944 & +0.00766822 & -0.000582775 & $+1.5051 \mathrm{e}-05$ \\
7 & -0.116057 & -0.0986929 & -0.0424235 & +0.00874221 & -0.000641953 & $+1.63221 \mathrm{e}-05$ \\
8 & -0.111262 & -0.0578045 & -0.0471432 & +0.0090904 & -0.000654415 & $+1.64714 \mathrm{e}-05$ \\
9 & -0.100128 & -0.0329076 & -0.047841 & +0.00890982 & -0.000633838 & $+1.58436 \mathrm{e}-05$ \\
10 & -0.0884076 & -0.0161829 & -0.0471498 & +0.00857635 & -0.000604769 & $+1.50385 \mathrm{e}-05$ \\
11 & -0.0809696 & -0.00252313 & -0.0462004 & +0.00822121 & -0.000574152 & $+1.41848 \mathrm{e}-05$ \\
12 & -0.0766755 & +0.00897778 & -0.0450013 & +0.00784999 & -0.00054377 & $+1.33703 \mathrm{e}-05$ \\
13 & -0.0738294 & +0.0187953 & -0.0439496 & +0.00752528 & -0.000516912 & $+1.26415 \mathrm{e}-05$ \\
14 & -0.0696698 & +0.0245555 & -0.0419045 & +0.00707475 & -0.000482703 & $+1.17531 \mathrm{e}-05$ \\
15 & -0.0650562 & +0.0282118 & -0.0396421 & +0.00660578 & -0.000447509 & $+1.08415 \mathrm{e}-05$ \\
\hline
\end{tabular}


Table 6.37: Fit result of Eq. (6.7) to different apertures and models, for the correction to infinite aperture, as a function of the intrinsic size of a given cluster. NICMOS/NIC2 chip, F160W filter, post-cooler

\begin{tabular}{|c|c|c|c|c|c|c|}
\hline \multicolumn{7}{|c|}{ EFF 15 } \\
\hline Aperture (pixels) & $a$ & $b$ & $c$ & $d$ & $e$ & $f$ \\
\hline 2 & -0.733948 & -0.454379 & +0.0276535 & -0.00102558 & $+1.24175 \mathrm{e}-05$ & $+2.05861 \mathrm{e}-07$ \\
3 & -0.454508 & -0.280109 & -0.00151979 & +0.00176219 & -0.000126855 & $+2.99707 \mathrm{e}-06$ \\
4 & -0.277022 & -0.21351 & -0.0018937 & +0.0010092 & $-6.06247 \mathrm{e}-05$ & $+1.2509 \mathrm{e}-06$ \\
5 & -0.198332 & -0.158667 & -0.003709 & +0.000809178 & $-4.09744 \mathrm{e}-05$ & $+7.53479 \mathrm{e}-07$ \\
6 & -0.174421 & -0.114304 & -0.00584883 & +0.000828527 & $-3.95794 \mathrm{e}-05$ & $+7.31627 \mathrm{e}-07$ \\
7 & -0.159351 & -0.0859797 & -0.00560108 & +0.000615948 & $-2.45131 \mathrm{e}-05$ & $+3.7199 \mathrm{e}-07$ \\
8 & -0.14389 & -0.0688668 & -0.00493846 & +0.000487698 & $-1.90749 \mathrm{e}-05$ & $+3.03781 \mathrm{e}-07$ \\
9 & -0.123865 & -0.0598378 & -0.00330482 & +0.000263002 & $-7.35516 \mathrm{e}-06$ & $+7.07 \mathrm{e}-08$ \\
10 & -0.105268 & -0.0535701 & -0.00209346 & +0.000119936 & $-4.6404 \mathrm{e}-07$ & $-6.26046 \mathrm{e}-08$ \\
11 & -0.0922637 & -0.0472381 & -0.00149172 & $+5.99516 \mathrm{e}-05$ & $+1.55087 \mathrm{e}-06$ & $-8.60866 \mathrm{e}-08$ \\
12 & -0.0834922 & -0.0401932 & -0.00151733 & $+8.06829 \mathrm{e}-05$ & $-8.65144 \mathrm{e}-07$ & $-1.80409 \mathrm{e}-08$ \\
13 & -0.0769328 & -0.0337724 & -0.00171631 & +0.000120903 & $-4.13095 \mathrm{e}-06$ & $+6.57939 \mathrm{e}-08$ \\
14 & -0.0698049 & -0.029292 & -0.00158474 & +0.000118487 & $-4.87524 \mathrm{e}-06$ & $+9.71434 \mathrm{e}-08$ \\
15 & -0.0629278 & -0.0260213 & -0.00138041 & +0.000107947 & $-4.99931 \mathrm{e}-06$ & $+1.0879 \mathrm{e}-07$ \\
\hline
\end{tabular}


Table 6.38: Fit result of Eq. (6.7) to different apertures and models, for the correction to infinite aperture, as a function of the intrinsic size of a given cluster. NICMOS/NIC2 chip, F205W filter, pre-cooler

\begin{tabular}{|c|c|c|c|c|c|c|}
\hline \multicolumn{7}{|c|}{ King 5 } \\
\hline Aperture (pixels) & $a$ & $b$ & $c$ & $d$ & $e$ & $f$ \\
\hline 2 & -1.00909 & -0.124986 & -0.0666072 & +0.0114361 & -0.000760508 & $+1.81868 \mathrm{e}-05$ \\
3 & -0.710809 & +0.0176839 & -0.0793071 & +0.011285 & -0.000685781 & $+1.54902 \mathrm{e}-05$ \\
4 & -0.521699 & +0.0612163 & -0.0663588 & +0.0081055 & -0.000442188 & $+9.19863 \mathrm{e}-06$ \\
5 & -0.352058 & +0.0408418 & -0.0429189 & +0.00424556 & -0.000186286 & $+3.10213 \mathrm{e}-06$ \\
6 & -0.25467 & +0.029025 & -0.0275923 & +0.00188606 & $-3.85015 \mathrm{e}-05$ & $-2.6262 \mathrm{e}-07$ \\
7 & -0.204382 & +0.0232087 & -0.0162135 & +0.000183646 & $+6.52204 \mathrm{e}-05$ & $-2.57397 \mathrm{e}-06$ \\
8 & -0.174129 & +0.013808 & -0.00654786 & -0.00112347 & +0.000138863 & $-4.10648 \mathrm{e}-06$ \\
9 & -0.151203 & +0.0037384 & +0.00106397 & -0.00202171 & +0.000183324 & $-4.91813 \mathrm{e}-06$ \\
10 & -0.133522 & -0.00323942 & +0.00537049 & -0.00235152 & +0.000189106 & $-4.79641 \mathrm{e}-06$ \\
11 & -0.117903 & -0.00771532 & +0.00734739 & -0.00231229 & +0.00017123 & $-4.12504 \mathrm{e}-06$ \\
12 & -0.103371 & -0.0113402 & +0.00826265 & -0.00212848 & +0.000145653 & $-3.30787 \mathrm{e}-06$ \\
13 & -0.0921492 & -0.011158 & +0.00735768 & -0.00171129 & +0.00010765 & $-2.25408 \mathrm{e}-06$ \\
14 & -0.0822074 & -0.0111256 & +0.00638629 & -0.00131429 & $+7.2521 \mathrm{e}-05$ & $-1.29257 \mathrm{e}-06$ \\
15 & -0.0756899 & -0.00885686 & +0.00473738 & -0.000881725 & $+3.8642 \mathrm{e}-05$ & $-4.28602 \mathrm{e}-07$ \\
\hline
\end{tabular}


Table 6.39: Fit result of Eq. (6.7) to different apertures and models, for the correction to infinite aperture, as a function of the intrinsic size of a given cluster. NICMOS/NIC2 chip, F205W filter, pre-cooler

\begin{tabular}{|c|c|c|c|c|c|c|}
\hline \multicolumn{7}{|c|}{ King 30 } \\
\hline Aperture (pixels) & $a$ & $b$ & $c$ & $d$ & $e$ & $f$ \\
\hline 2 & -0.968433 & -0.674762 & +0.0802667 & -0.00616122 & +0.000251705 & $-4.15115 \mathrm{e}-06$ \\
3 & -0.625478 & -0.458694 & +0.0278431 & +0.000498052 & -0.000155433 & $+5.28036 \mathrm{e}-06$ \\
4 & -0.427815 & -0.318126 & -0.00235128 & +0.00417208 & -0.000376285 & $+1.03557 \mathrm{e}-05$ \\
5 & -0.274191 & -0.248818 & -0.0127638 & +0.00521528 & -0.000432392 & $+1.1551 \mathrm{e}-05$ \\
6 & -0.190178 & -0.188625 & -0.0224936 & +0.00627074 & -0.000492474 & $+1.28881 \mathrm{e}-05$ \\
7 & -0.151192 & -0.129804 & -0.0324927 & +0.0073676 & -0.000553936 & $+1.42224 \mathrm{e}-05$ \\
8 & -0.132769 & -0.0853951 & -0.0388149 & +0.00796113 & -0.000583025 & $+1.47826 \mathrm{e}-05$ \\
9 & -0.121695 & -0.0509778 & -0.0424685 & +0.0081749 & -0.000586756 & $+1.47175 \mathrm{e}-05$ \\
10 & -0.113771 & -0.0262325 & -0.0441106 & +0.00815717 & -0.000577772 & $+1.43919 \mathrm{e}-05$ \\
11 & -0.105842 & -0.00918951 & -0.0441627 & +0.00794293 & -0.000556535 & $+1.3771 \mathrm{e}-05$ \\
12 & -0.0974414 & +0.00172636 & -0.0427314 & +0.00753305 & -0.000523254 & $+1.28761 \mathrm{e}-05$ \\
13 & -0.0900841 & +0.0105482 & -0.0414133 & +0.00717902 & -0.000495101 & $+1.21301 \mathrm{e}-05$ \\
14 & -0.0835314 & +0.0161415 & -0.039119 & +0.00667024 & -0.000456024 & $+1.11047 \mathrm{e}-05$ \\
15 & -0.0786844 & +0.0208743 & -0.0372243 & +0.0062608 & -0.000425156 & $+1.03072 \mathrm{e}-05$ \\
\hline
\end{tabular}


Table 6.40: Fit result of Eq. (6.7) to different apertures and models, for the correction to infinite aperture, as a function of the intrinsic size of a given cluster. NICMOS/NIC2 chip, F205W filter, pre-cooler

\begin{tabular}{|c|c|c|c|c|c|c|}
\hline \multicolumn{7}{|c|}{ EFF 15 } \\
\hline Aperture (pixels) & $a$ & $b$ & $c$ & $d$ & $e$ & $f$ \\
\hline 2 & -1.0449 & -0.442492 & +0.0336258 & -0.00203999 & $+7.69346 \mathrm{e}-05$ & $-1.2728 \mathrm{e}-06$ \\
3 & -0.708916 & -0.276391 & +0.00421482 & +0.000890089 & $-7.39066 \mathrm{e}-05$ & $+1.81827 \mathrm{e}-06$ \\
4 & -0.50473 & -0.189581 & -0.00460379 & +0.00134907 & $-8.39169 \mathrm{e}-05$ & $+1.84248 \mathrm{e}-06$ \\
5 & -0.338639 & -0.163419 & -0.000248858 & +0.000374645 & $-1.64835 \mathrm{e}-05$ & $+2.29157 \mathrm{e}-07$ \\
6 & -0.244113 & -0.135636 & -0.00015448 & +0.000203461 & $-5.86683 \mathrm{e}-06$ & $+1.78395 \mathrm{e}-08$ \\
7 & -0.1951 & -0.106409 & -0.00148472 & +0.000239047 & $-7.03153 \mathrm{e}-06$ & $+4.95307 \mathrm{e}-08$ \\
8 & -0.167744 & -0.0842307 & -0.00238338 & +0.000288228 & $-1.11613 \mathrm{e}-05$ & $+1.84 \mathrm{e}-07$ \\
9 & -0.148025 & -0.0686361 & -0.00215165 & +0.000202329 & $-6.44021 \mathrm{e}-06$ & $+9.31309 \mathrm{e}-08$ \\
10 & -0.132647 & -0.057154 & -0.00188215 & +0.000138358 & $-2.8637 \mathrm{e}-06$ & $+1.35467 \mathrm{e}-08$ \\
11 & -0.119086 & -0.0486697 & -0.00155232 & $+8.44441 \mathrm{e}-05$ & $-1.23247 \mathrm{e}-07$ & $-4.27736 \mathrm{e}-08$ \\
12 & -0.105985 & -0.0429211 & -0.00106499 & $+3.46526 \mathrm{e}-05$ & $+1.49008 \mathrm{e}-06$ & $-5.774 \mathrm{e}-08$ \\
13 & -0.0945485 & -0.0384903 & -0.000577268 & $-1.84419 \mathrm{e}-05$ & $+3.82346 \mathrm{e}-06$ & $-9.96325 \mathrm{e}-08$ \\
14 & -0.085079 & -0.0341664 & -0.000352666 & $-3.61532 \mathrm{e}-05$ & $+4.17618 \mathrm{e}-06$ & $-9.81621 \mathrm{e}-08$ \\
15 & -0.0779083 & -0.0302092 & -0.000189085 & $-5.77568 \mathrm{e}-05$ & $+5.59425 \mathrm{e}-06$ & $-1.39141 \mathrm{e}-07$ \\
\hline
\end{tabular}


Table 6.41: Fit result of Eq. (6.7) to different apertures and models, for the correction to infinite aperture, as a function of the intrinsic size of a given cluster. NICMOS/NIC2 chip, F205W filter, post-cooler

\begin{tabular}{|c|c|c|c|c|c|c|}
\hline \multicolumn{7}{|c|}{ King 5 } \\
\hline Aperture (pixels) & $a$ & $b$ & $c$ & $d$ & $e$ & $f$ \\
\hline 2 & -0.827785 & -0.136113 & -0.0701698 & +0.0121567 & -0.000810264 & $+1.93825 \mathrm{e}-05$ \\
3 & -0.599621 & +0.0332773 & -0.0873625 & +0.012378 & -0.000749947 & $+1.68843 \mathrm{e}-05$ \\
4 & -0.428818 & +0.0666931 & -0.0694684 & +0.00844091 & -0.000456919 & $+9.4193 \mathrm{e}-06$ \\
5 & -0.272884 & +0.0386489 & -0.0429836 & +0.00417618 & -0.000177383 & $+2.8117 \mathrm{e}-06$ \\
6 & -0.205021 & +0.0314622 & -0.027783 & +0.00177964 & $-2.57417 \mathrm{e}-05$ & $-6.64818 \mathrm{e}-07$ \\
7 & -0.178978 & +0.0266299 & -0.0155562 & $-7.31463 \mathrm{e}-05$ & $+8.76428 \mathrm{e}-05$ & $-3.19366 \mathrm{e}-06$ \\
8 & -0.161895 & +0.0147054 & -0.0044943 & -0.00159143 & +0.000175232 & $-5.06763 \mathrm{e}-06$ \\
9 & -0.14705 & +0.00287386 & +0.00365756 & -0.00252792 & +0.000219944 & $-5.8433 \mathrm{e}-06$ \\
10 & -0.13119 & -0.00616139 & +0.0082572 & -0.00285384 & +0.000223417 & $-5.63003 \mathrm{e}-06$ \\
11 & -0.115255 & -0.0109991 & +0.00979768 & -0.00271196 & +0.000197701 & $-4.75954 \mathrm{e}-06$ \\
12 & -0.0982696 & -0.0146543 & +0.00998443 & -0.00236827 & +0.000159871 & $-3.62445 \mathrm{e}-06$ \\
13 & -0.0843955 & -0.015047 & +0.00883911 & -0.00189187 & +0.000117244 & $-2.44751 \mathrm{e}-06$ \\
14 & -0.0753546 & -0.0126443 & +0.00690505 & -0.00136548 & $+7.51184 \mathrm{e}-05$ & $-1.35754 \mathrm{e}-06$ \\
15 & -0.0698115 & -0.00898933 & +0.00470892 & -0.000853802 & $+3.61711 \mathrm{e}-05$ & $-3.71414 \mathrm{e}-07$ \\
\hline
\end{tabular}


Table 6.42: Fit result of Eq. (6.7) to different apertures and models, for the correction to infinite aperture, as a function of the intrinsic size of a given cluster. NICMOS/NIC2 chip, F205W filter, post-cooler

\begin{tabular}{|c|c|c|c|c|c|c|}
\hline \multicolumn{7}{|c|}{ King 30 } \\
\hline Aperture (pixels) & $a$ & $b$ & $c$ & $d$ & $e$ & $f$ \\
\hline 2 & -0.79017 & -0.706263 & +0.0844539 & -0.00655122 & +0.000271214 & $-4.53177 \mathrm{e}-06$ \\
3 & -0.507939 & -0.460871 & +0.0246812 & +0.00104877 & -0.000193177 & $+6.21266 \mathrm{e}-06$ \\
4 & -0.332413 & -0.322529 & -0.00355388 & +0.0044029 & -0.000392531 & $+1.07651 \mathrm{e}-05$ \\
5 & -0.195739 & -0.255618 & -0.0125259 & +0.00524171 & -0.000436348 & $+1.1689 \mathrm{e}-05$ \\
6 & -0.139947 & -0.185732 & -0.0246598 & +0.00661624 & -0.000516256 & $+1.34844 \mathrm{e}-05$ \\
7 & -0.125244 & -0.119831 & -0.0362004 & +0.00791027 & -0.00059009 & $+1.51124 \mathrm{e}-05$ \\
8 & -0.121716 & -0.0728606 & -0.0428033 & +0.00851303 & -0.00061835 & $+1.56256 \mathrm{e}-05$ \\
9 & -0.119927 & -0.0376656 & -0.0465471 & +0.00875241 & -0.000624889 & $+1.56551 \mathrm{e}-05$ \\
10 & -0.114948 & -0.0148468 & -0.0473987 & +0.00860059 & -0.000605712 & $+1.50494 \mathrm{e}-05$ \\
11 & -0.10632 & -0.00054284 & -0.0466533 & +0.00828488 & -0.000578704 & $+1.43103 \mathrm{e}-05$ \\
12 & -0.0956333 & +0.00792619 & -0.044608 & +0.00779713 & -0.00054057 & $+1.32983 \mathrm{e}-05$ \\
13 & -0.0858676 & +0.0135643 & -0.042241 & +0.007287 & -0.000501919 & $+1.22937 \mathrm{e}-05$ \\
14 & -0.0789868 & +0.0191093 & -0.0401907 & +0.0068376 & -0.000467816 & $+1.14078 \mathrm{e}-05$ \\
15 & -0.0742885 & +0.0240402 & -0.0383798 & +0.00643726 & -0.000437274 & $+1.06112 \mathrm{e}-05$ \\
\hline
\end{tabular}


Table 6.43: Fit result of Eq. (6.7) to different apertures and models, for the correction to infinite aperture, as a function of the intrinsic size of a given cluster. NICMOS/NIC2 chip, F205W filter, post-cooler

\begin{tabular}{|c|c|c|c|c|c|c|}
\hline \multicolumn{7}{|c|}{ EFF 15 } \\
\hline Aperture (pixels) & $a$ & $b$ & $c$ & $d$ & $e$ & $f$ \\
\hline 2 & -0.867243 & -0.463586 & +0.034491 & -0.00202033 & $+7.43707 \mathrm{e}-05$ & $-1.22292 \mathrm{e}-06$ \\
3 & -0.594389 & -0.27188 & -0.000308128 & +0.00153399 & -0.000112908 & $+2.69682 \mathrm{e}-06$ \\
4 & -0.410953 & -0.190427 & -0.00600065 & +0.00150437 & $-9.07753 \mathrm{e}-05$ & $+1.95359 \mathrm{e}-06$ \\
5 & -0.26045 & -0.168242 & $+2.44677 \mathrm{e}-05$ & +0.000323982 & $-1.19744 \mathrm{e}-05$ & $+9.78824 \mathrm{e}-08$ \\
6 & -0.194143 & -0.13358 & -0.00115125 & +0.000303977 & $-1.05954 \mathrm{e}-05$ & $+1.05677 \mathrm{e}-07$ \\
7 & -0.169176 & -0.100011 & -0.00301235 & +0.000391152 & $-1.4818 \mathrm{e}-05$ & $+2.12362 \mathrm{e}-07$ \\
8 & -0.155147 & -0.0789254 & -0.00291075 & +0.000268126 & $-6.51701 \mathrm{e}-06$ & $+2.12778 \mathrm{e}-08$ \\
9 & -0.143897 & -0.0628673 & -0.00280352 & +0.000207254 & $-3.50035 \mathrm{e}-06$ & $-3.10287 \mathrm{e}-08$ \\
10 & -0.132008 & -0.0518707 & -0.00264014 & +0.000196093 & $-5.27061 \mathrm{e}-06$ & $+5.54863 \mathrm{e}-08$ \\
11 & -0.117292 & -0.0458915 & -0.00184923 & +0.00011103 & $-1.9877 \mathrm{e}-06$ & $+1.01844 \mathrm{e}-08$ \\
12 & -0.10199 & -0.0421257 & -0.000840903 & $-2.4225 \mathrm{e}-05$ & $+6.39715 \mathrm{e}-06$ & $-1.97413 \mathrm{e}-07$ \\
13 & -0.0891172 & -0.0377878 & -0.000693658 & $-4.04201 \mathrm{e}-06$ & $+3.09536 \mathrm{e}-06$ & $-8.95124 \mathrm{e}-08$ \\
14 & -0.0789567 & -0.034243 & -0.000254907 & $-5.20176 \mathrm{e}-05$ & $+5.38969 \mathrm{e}-06$ & $-1.33556 \mathrm{e}-07$ \\
15 & -0.0723542 & -0.0291839 & -0.000613778 & $+1.21042 \mathrm{e}-05$ & $+8.51247 \mathrm{e}-07$ & $-2.58395 \mathrm{e}-08$ \\
\hline
\end{tabular}


6.8.3 Parameters of aperture correction fits: Measured sizes 
Table 6.44: Fit result of Eq. (6.7) to different apertures and models, for the correction to infinite aperture, as a function of the measured size of a cluster. PC chip, F555W filter

\begin{tabular}{|c|c|c|c|c|c|c|}
\hline \multicolumn{7}{|c|}{ King 5 } \\
\hline Aperture (pixels) & $a$ & $b$ & $c$ & $d$ & $e$ & $f$ \\
\hline 2 & -0.660914 & +0.412501 & -0.190855 & +0.0183306 & -0.00067422 & $+6.84224 \mathrm{E}-06$ \\
3 & -0.54114 & +0.318153 & -0.0888606 & -0.000710807 & +0.000766066 & $-3.26733 \mathrm{E}-05$ \\
4 & -0.276386 & +0.00476915 & +0.0556084 & -0.0232744 & +0.00232172 & $-7.28605 \mathrm{E}-05$ \\
5 & 0.0527368 & -0.320821 & +0.173947 & -0.0394022 & +0.00331916 & $-9.63591 \mathrm{E}-05$ \\
6 & 0.284994 & -0.521061 & +0.235929 & -0.0462376 & +0.00364481 & -0.000101877 \\
7 & 0.393397 & -0.595175 & +0.250633 & -0.0457873 & +0.00346078 & $-9.39791 \mathrm{E}-05$ \\
8 & 0.421499 & -0.592066 & +0.238708 & -0.0416676 & +0.00304688 & $-8.06268 \mathrm{E}-05$ \\
9 & 0.401889 & -0.539891 & +0.210017 & -0.0352803 & +0.00249563 & $-6.41715 \mathrm{E}-05$ \\
10 & 0.338736 & -0.449241 & +0.169912 & -0.0275815 & +0.00187786 & $-4.64806 \mathrm{E}-05$ \\
11 & 0.275995 & -0.362963 & +0.132905 & -0.0207161 & +0.00134029 & $-3.13695 \mathrm{E}-05$ \\
12 & 0.200507 & -0.270108 & +0.0956521 & -0.0141742 & +0.000847138 & $-1.79042 \mathrm{E}-05$ \\
13 & 0.136277 & -0.190463 & +0.0640847 & -0.00874388 & +0.000445678 & $-7.12379 \mathrm{E}-06$ \\
14 & 0.0798999 & -0.122817 & +0.0381835 & -0.00444161 & +0.000139135 & $+8.02809 \mathrm{E}-07$ \\
15 & 0.0252033 & -0.0598383 & +0.0148455 & -0.000689303 & -0.000121051 & $+7.37615 \mathrm{E}-06$ \\
\hline
\end{tabular}


Table 6.45: Fit result of Eq. (6.7) to different apertures and models, for the correction to infinite aperture, as a function of the measured size of a cluster. PC chip, F555W filter

\begin{tabular}{|c|c|c|c|c|c|c|}
\hline \multicolumn{7}{|c|}{ King 30 } \\
\hline Aperture (pixels) & $a$ & $b$ & $c$ & $d$ & $e$ & $f$ \\
\hline 2 & -1.79185 & +1.95995 & -0.860534 & +0.129127 & -0.00868911 & +0.000221397 \\
3 & -2.25942 & +2.38604 & -0.926448 & +0.135546 & -0.00907912 & +0.000232076 \\
4 & -2.63607 & +2.62935 & -0.953106 & +0.137746 & -0.00923589 & +0.000237429 \\
5 & -2.71478 & +2.62667 & -0.920093 & +0.132162 & -0.00886653 & +0.000228516 \\
6 & -2.63599 & +2.50722 & -0.861467 & +0.123249 & -0.0082654 & +0.000213139 \\
7 & -2.50966 & +2.35154 & -0.795439 & +0.113232 & -0.00757363 & +0.000194908 \\
8 & -2.34768 & +2.17028 & -0.724246 & +0.102363 & -0.00680783 & +0.000174291 \\
9 & -2.15111 & +1.96764 & -0.649321 & +0.0911012 & -0.00602045 & +0.000153227 \\
10 & -1.94852 & +1.76577 & -0.576629 & +0.0801705 & -0.00525181 & +0.000132523 \\
11 & -1.75901 & +1.57976 & -0.510569 & +0.0702687 & -0.0045563 & +0.000113807 \\
12 & -1.58747 & +1.41323 & -0.452247 & +0.0616305 & -0.00395693 & $+9.788 \mathrm{E}-05$ \\
13 & -1.42506 & +1.25901 & -0.399192 & +0.0538325 & -0.00341817 & $+8.35955 \mathrm{E}-05$ \\
14 & -1.26147 & +1.10433 & -0.346134 & +0.045979 & -0.00287128 & $+6.89947 \mathrm{E}-05$ \\
15 & -1.13463 & +0.985699 & -0.306045 & +0.0401902 & -0.00247806 & $+5.87332 \mathrm{E}-05$ \\
\hline
\end{tabular}


Table 6.46: Fit result of Eq. (6.7) to different apertures and models, for the correction to infinite aperture, as a function of the measured size of a cluster. PC chip, F555W filter

\begin{tabular}{|c|c|c|c|c|c|c|}
\hline \multicolumn{7}{|c|}{ EFF 15 } \\
\hline Aperture (pixels) & $a$ & $b$ & $c$ & $d$ & $e$ & $f$ \\
\hline 2 & 0.101597 & -0.0617422 & -0.13958 & +0.0191528 & -0.00101713 & $+1.93721 \mathrm{E}-05$ \\
3 & -0.0621851 & +0.11108 & -0.125518 & +0.0134571 & -0.000528562 & $+5.62916 \mathrm{E}-06$ \\
4 & -0.144039 & +0.124466 & -0.0854923 & +0.00623307 & $-3.50479 \mathrm{E}-05$ & $-6.49834 \mathrm{E}-06$ \\
5 & -0.0927692 & +0.0488902 & -0.0409979 & +0.000133943 & +0.000314034 & $-1.38201 \mathrm{E}-05$ \\
6 & -0.0356313 & -0.00817004 & -0.014672 & -0.00276277 & +0.000437269 & $-1.53908 \mathrm{E}-05$ \\
7 & -0.0165412 & -0.0291032 & -0.00303759 & -0.00362572 & +0.000439272 & $-1.43106 \mathrm{E}-05$ \\
8 & -0.0146819 & -0.0312521 & +0.000221029 & -0.00338207 & +0.000378642 & $-1.19103 \mathrm{E}-05$ \\
9 & -0.0123202 & -0.0309367 & +0.00175231 & -0.0030178 & +0.000321584 & $-9.88691 \mathrm{E}-06$ \\
10 & -0.0071123 & -0.03077 & +0.00230867 & -0.00257191 & +0.000263022 & $-7.87359 \mathrm{E}-06$ \\
11 & -0.0116445 & -0.0223694 & +0.00019288 & -0.00187052 & +0.000195992 & $-5.83465 \mathrm{E}-06$ \\
12 & -0.00813437 & -0.0233257 & +0.00153477 & -0.00182905 & +0.000183829 & $-5.43802 \mathrm{E}-06$ \\
13 & -0.0113161 & -0.0170268 & $+3.18708 \mathrm{E}-06$ & -0.00136581 & +0.000142033 & $-4.22121 \mathrm{E}-06$ \\
14 & -0.0139007 & -0.0112987 & -0.00147441 & -0.000936712 & +0.00010389 & $-3.1214 \mathrm{E}-06$ \\
15 & -0.0118029 & -0.0104534 & -0.00136931 & -0.000777288 & $+8.48166 \mathrm{E}-05$ & $-2.48327 \mathrm{E}-06$ \\
\hline
\end{tabular}


Table 6.47: Fit result of Eq. (6.7) to different apertures and models, for the correction to infinite aperture, as a function of the measured size of a cluster. WF3 chip, F555W filter

\begin{tabular}{|c|c|c|c|c|c|c|}
\hline \multicolumn{7}{|c|}{ King 5 } \\
\hline Aperture (pixels) & $a$ & $b$ & $c$ & $d$ & $e$ & $f$ \\
\hline 2 & -0.678284 & +0.489979 & -0.218123 & +0.0224476 & -0.000971317 & $+1.51631 \mathrm{E}-05$ \\
3 & -0.520845 & +0.363595 & -0.105187 & +0.00161789 & +0.000607791 & $-2.84509 \mathrm{E}-05$ \\
4 & -0.203761 & +0.00785934 & +0.0525285 & -0.0229136 & +0.00230346 & $-7.24802 \mathrm{E}-05$ \\
5 & 0.0834688 & -0.289561 & +0.16345 & -0.0380944 & +0.00324296 & $-9.4625 \mathrm{E}-05$ \\
6 & 0.264027 & -0.45638 & +0.21616 & -0.0436021 & +0.00347388 & $-9.75032 \mathrm{E}-05$ \\
7 & 0.347541 & -0.512145 & +0.224791 & -0.042163 & +0.00321398 & $-8.7418 \mathrm{E}-05$ \\
8 & 0.362666 & -0.497524 & +0.208122 & -0.0371469 & +0.00272412 & $-7.17118 \mathrm{E}-05$ \\
9 & 0.333532 & -0.439403 & +0.176846 & -0.0302172 & +0.002123 & $-5.36127 \mathrm{E}-05$ \\
10 & 0.278828 & -0.360897 & +0.140408 & -0.0229588 & +0.0015276 & $-3.62966 \mathrm{E}-05$ \\
11 & 0.219309 & -0.280452 & +0.104876 & -0.016226 & +0.0009939 & $-2.11677 \mathrm{E}-05$ \\
12 & 0.154239 & -0.198197 & +0.0703799 & -0.00998871 & +0.000515741 & $-7.96413 \mathrm{E}-06$ \\
13 & 0.09792 & -0.127794 & +0.0415962 & -0.00494001 & +0.000140016 & $+2.12392 \mathrm{E}-06$ \\
14 & 0.051061 & -0.0699366 & +0.0185494 & -0.00102533 & -0.000140831 & $+9.38271 \mathrm{E}-06$ \\
15 & 0.0128557 & -0.0234692 & +0.000516643 & +0.00194263 & -0.000345926 & $+1.44674 \mathrm{E}-05$ \\
\hline
\end{tabular}


Table 6.48: Fit result of Eq. (6.7) to different apertures and models, for the correction to infinite aperture, as a function of the measured size of a cluster. WF3 chip, F555W filter

\begin{tabular}{|c|c|c|c|c|c|c|}
\hline \multicolumn{7}{|c|}{ King 30 } \\
\hline Aperture (pixels) & $a$ & $b$ & $c$ & $d$ & $e$ & $f$ \\
\hline 2 & -1.48486 & +1.72034 & -0.781068 & +0.116102 & -0.00764245 & +0.000188649 \\
3 & -1.99789 & +2.20123 & -0.868263 & +0.126263 & -0.00834 & +0.000208911 \\
4 & -2.28948 & +2.39106 & -0.882108 & +0.127079 & -0.00843495 & +0.000213594 \\
5 & -2.38265 & +2.40546 & -0.856241 & +0.122881 & -0.00819103 & +0.00020892 \\
6 & -2.33809 & +2.30984 & -0.804295 & +0.114912 & -0.00765726 & +0.000195477 \\
7 & -2.21033 & +2.14933 & -0.735909 & +0.104439 & -0.00692683 & +0.000176048 \\
8 & -2.05979 & +1.97861 & -0.66855 & +0.094201 & -0.00620879 & +0.000156785 \\
9 & -1.87339 & +1.77785 & -0.592421 & +0.0824943 & -0.0053717 & +0.000133903 \\
10 & -1.70006 & +1.59413 & -0.524157 & +0.0720706 & -0.00462978 & +0.000113704 \\
11 & -1.52497 & +1.41483 & -0.459317 & +0.0622562 & -0.00393567 & $+9.49368 \mathrm{E}-05$ \\
12 & -1.36003 & +1.24787 & -0.399612 & +0.0532495 & -0.00329971 & $+7.77564 \mathrm{E}-05$ \\
13 & -1.20427 & +1.09316 & -0.344983 & +0.045026 & -0.00271828 & $+6.19967 \mathrm{E}-05$ \\
14 & -1.05245 & +0.943919 & -0.292809 & +0.0372087 & -0.00216874 & $+4.72173 \mathrm{E}-05$ \\
15 & -0.929427 & +0.825577 & -0.252428 & +0.0313432 & -0.0017691 & $+3.67891 \mathrm{E}-05$ \\
\hline
\end{tabular}


Table 6.49: Fit result of Eq. (6.7) to different apertures and models, for the correction to infinite aperture, as a function of the measured size of a cluster. WF3 chip, F555W filter

\begin{tabular}{|c|c|c|c|c|c|c|}
\hline \multicolumn{7}{|c|}{ EFF 15 } \\
\hline Aperture (pixels) & $a$ & $b$ & $c$ & $d$ & $e$ & $f$ \\
\hline 2 & -0.197547 & +0.235124 & -0.23533 & +0.0334999 & -0.00203347 & $+4.6805 \mathrm{E}-05$ \\
3 & -0.284494 & +0.347447 & -0.202682 & +0.0250798 & -0.00135464 & $+2.79765 \mathrm{E}-05$ \\
4 & -0.230654 & +0.252511 & -0.1295 & +0.0130301 & -0.000526366 & $+6.94963 \mathrm{E}-06$ \\
5 & -0.145742 & +0.143849 & -0.0735813 & +0.00512575 & $-4.44828 \mathrm{E}-05$ & $-4.05185 \mathrm{E}-06$ \\
6 & -0.0890236 & +0.0785805 & -0.0432653 & +0.00151459 & +0.000134323 & $-7.2046 \mathrm{E}-06$ \\
7 & -0.0545739 & +0.0428715 & -0.0271714 & $+3.99231 \mathrm{E}-05$ & +0.000175742 & $-7.0894 \mathrm{E}-06$ \\
8 & -0.0432793 & +0.0326118 & -0.0218415 & $+5.10128 \mathrm{E}-05$ & +0.000126706 & $-4.88752 \mathrm{E}-06$ \\
9 & -0.0338311 & +0.0243649 & -0.017595 & $+2.71394 \mathrm{E}-05$ & $+9.56854 \mathrm{E}-05$ & $-3.52748 \mathrm{E}-06$ \\
10 & -0.0269923 & +0.0178575 & -0.0141916 & $-3.10281 \mathrm{E}-05$ & $+7.74326 \mathrm{E}-05$ & $-2.70764 \mathrm{E}-06$ \\
11 & -0.0211795 & +0.0122217 & -0.0112887 & -0.000120992 & $+6.82416 \mathrm{E}-05$ & $-2.26488 \mathrm{E}-06$ \\
12 & -0.0190346 & +0.0108055 & -0.0101086 & $-3.68288 \mathrm{E}-06$ & $+4.75348 \mathrm{E}-05$ & $-1.56234 \mathrm{E}-06$ \\
13 & -0.0130122 & +0.00629541 & -0.00799982 & $-9.31764 \mathrm{E}-05$ & $+4.48676 \mathrm{E}-05$ & $-1.39505 \mathrm{E}-06$ \\
14 & -0.00965565 & +0.00363862 & -0.00636775 & -0.000176703 & $+4.53483 \mathrm{E}-05$ & $-1.37381 \mathrm{E}-06$ \\
15 & -0.0140032 & +0.0092721 & -0.00803789 & +0.000274421 & $+5.21679 \mathrm{E}-06$ & $-1.87418 \mathrm{E}-07$ \\
\hline
\end{tabular}


Table 6.50: Fit result of Eq. (6.7) to different apertures and models, for the correction to infinite aperture, as a function of the measured size of a cluster. ACS/WFC1 chip, F555W filter (chip 2 is equivalent)

\begin{tabular}{|c|c|c|c|c|c|c|}
\hline \multicolumn{7}{|c|}{ King 5 } \\
\hline Aperture (pixels) & $a$ & $b$ & $c$ & $d$ & $e$ & $f$ \\
\hline 2 & -0.605465 & +0.405731 & -0.184549 & +0.0163657 & -0.000467535 & $-3.83373 \mathrm{E}-07$ \\
3 & -0.531879 & +0.347251 & -0.0924309 & -0.00147139 & +0.000909199 & $-3.88142 \mathrm{E}-05$ \\
4 & -0.237375 & -0.0102443 & +0.0691531 & -0.0268848 & +0.00268316 & $-8.53165 \mathrm{E}-05$ \\
5 & 0.160803 & -0.411283 & +0.213231 & -0.0469351 & +0.00395927 & -0.000116335 \\
6 & 0.429767 & -0.652043 & +0.287725 & -0.0554289 & +0.00438443 & -0.000124052 \\
7 & 0.550736 & -0.737681 & +0.30443 & -0.0549849 & +0.00418122 & -0.000115152 \\
8 & 0.579634 & -0.729288 & +0.288078 & -0.0498133 & +0.00366835 & $-9.85364 \mathrm{E}-05$ \\
9 & 0.544282 & -0.658951 & +0.251184 & -0.0418416 & +0.00298156 & $-7.78368 \mathrm{E}-05$ \\
10 & 0.472334 & -0.55966 & +0.206908 & -0.0332871 & +0.00228748 & $-5.76827 \mathrm{E}-05$ \\
11 & 0.368801 & -0.436273 & +0.156758 & -0.0242756 & +0.00158723 & $-3.79266 \mathrm{E}-05$ \\
12 & 0.279341 & -0.330165 & +0.114343 & -0.0168381 & +0.00102349 & $-2.23878 \mathrm{E}-05$ \\
13 & 0.175379 & -0.215739 & +0.0708784 & -0.00952011 & +0.000482423 & $-7.70769 \mathrm{E}-06$ \\
14 & 0.104509 & -0.136223 & +0.0409379 & -0.00459289 & +0.000131678 & $+1.38804 \mathrm{E}-06$ \\
15 & 0.0293846 & -0.0553976 & +0.0113974 & +0.000159634 & -0.000203867 & $+1.01095 \mathrm{E}-05$ \\
\hline
\end{tabular}


Table 6.51: Fit result of Eq. (6.7) to different apertures and models, for the correction to infinite aperture, as a function of the measured size of a cluster. ACS/WFC1 chip, F555W filter (chip 2 is equivalent)

\begin{tabular}{|c|c|c|c|c|c|c|}
\hline \multicolumn{7}{|c|}{ King 30 } \\
\hline Aperture (pixels) & $a$ & $b$ & $c$ & $d$ & $e$ & $f$ \\
\hline 2 & -2.05706 & +2.32481 & -0.995377 & +0.150924 & -0.0103197 & +0.000267453 \\
3 & -2.66392 & +2.85046 & -1.08861 & +0.160982 & -0.0109468 & +0.000284223 \\
4 & -3.14738 & +3.15196 & -1.12795 & +0.164578 & -0.0111826 & +0.000291458 \\
5 & -3.26459 & +3.15303 & -1.09155 & +0.158111 & -0.0107351 & +0.000280182 \\
6 & -3.21391 & +3.03587 & -1.03035 & +0.148508 & -0.0100687 & +0.000262658 \\
7 & -3.06675 & +2.84699 & -0.951271 & +0.136269 & -0.00920156 & +0.000239186 \\
8 & -2.87027 & +2.63132 & -0.868785 & +0.123669 & -0.00830821 & +0.000214931 \\
9 & -2.62309 & +2.37919 & -0.777204 & +0.109782 & -0.00732331 & +0.000188157 \\
10 & -2.37201 & +2.12943 & -0.688023 & +0.0961961 & -0.00635123 & +0.000161484 \\
11 & -2.15051 & +1.91277 & -0.612048 & +0.0847926 & -0.00554665 & +0.000139708 \\
12 & -1.92157 & +1.69304 & -0.536131 & +0.0734145 & -0.00474356 & +0.000117958 \\
13 & -1.73164 & +1.51155 & -0.473879 & +0.0641756 & -0.00409806 & +0.000100664 \\
14 & -1.52673 & +1.31956 & -0.408943 & +0.0545394 & -0.00342248 & $+8.24632 \mathrm{E}-05$ \\
15 & -1.35096 & +1.15507 & -0.353413 & +0.0463132 & -0.00284695 & $+6.69976 \mathrm{E}-05$ \\
\hline
\end{tabular}


Table 6.52: Fit result of Eq. (6.7) to different apertures and models, for the correction to infinite aperture, as a function of the measured size of a cluster. ACS/WFC1 chip, F555W filter (chip 2 is equivalent)

\begin{tabular}{|c|c|c|c|c|c|c|}
\hline \multicolumn{7}{|c|}{ EFF 15 } \\
\hline Aperture (pixels) & $a$ & $b$ & $c$ & $d$ & $e$ & $f$ \\
\hline 2 & 0.254125 & -0.0806219 & -0.145731 & +0.0207596 & -0.0011443 & $+2.25791 \mathrm{E}-05$ \\
3 & 0.0146208 & +0.141969 & -0.143546 & +0.0163661 & -0.000721221 & $+1.00284 \mathrm{E}-05$ \\
4 & -0.0968967 & +0.15407 & -0.0984515 & +0.00797907 & -0.000127955 & $-5.08553 \mathrm{E}-06$ \\
5 & -0.0461426 & +0.0565245 & -0.0437275 & +0.000105961 & +0.000355886 & $-1.61674 \mathrm{E}-05$ \\
6 & 0.00971629 & -0.0166758 & -0.0101615 & -0.00399342 & +0.000566737 & $-2.00894 \mathrm{E}-05$ \\
7 & 0.0353513 & -0.0506159 & +0.00571659 & -0.0054205 & +0.00060225 & $-1.97301 \mathrm{E}-05$ \\
8 & 0.0481175 & -0.0650731 & +0.0125677 & -0.00562417 & +0.000566235 & $-1.77953 \mathrm{E}-05$ \\
9 & 0.0459079 & -0.0640927 & +0.0139041 & -0.00522113 & +0.000506025 & $-1.56753 \mathrm{E}-05$ \\
10 & 0.0513706 & -0.0687407 & +0.0164345 & -0.00510255 & +0.000471739 & $-1.4315 \mathrm{E}-05$ \\
11 & 0.0517681 & -0.068568 & +0.0172261 & -0.00484583 & +0.000436163 & $-1.31137 \mathrm{E}-05$ \\
12 & 0.0466837 & -0.0631342 & +0.0160954 & -0.00436298 & +0.000387928 & $-1.16125 \mathrm{E}-05$ \\
13 & 0.0373745 & -0.0546726 & +0.0140392 & -0.00381713 & +0.000339056 & $-1.01529 \mathrm{E}-05$ \\
14 & 0.0320358 & -0.048908 & +0.0125807 & -0.00337378 & +0.000297868 & $-8.89601 \mathrm{E}-06$ \\
15 & 0.0339616 & -0.0500935 & +0.0134216 & -0.0033325 & +0.000288165 & $-8.5427 \mathrm{E}-06$ \\
\hline
\end{tabular}


Table 6.53: Fit result of Eq. (6.7) to different apertures and models, for the correction to infinite aperture, as a function of the measured size of a cluster. NICMOS/NIC2 chip, F110W filter, pre-cooler

\begin{tabular}{|c|c|c|c|c|c|c|}
\hline \multicolumn{7}{|c|}{ King 5 } \\
\hline Aperture (pixels) & $a$ & $b$ & $c$ & $d$ & $e$ & $f$ \\
\hline 2 & 3.00186 & -2.27048 & +0.54327 & -0.0757458 & +0.00505464 & -0.00012722 \\
3 & 0.305277 & -0.396321 & +0.116481 & -0.0263453 & +0.00221342 & $-6.28589 \mathrm{E}-05$ \\
4 & 0.221114 & -0.406227 & +0.165547 & -0.0349719 & +0.00280727 & $-7.76987 \mathrm{E}-05$ \\
5 & 0.483926 & -0.658405 & +0.258397 & -0.047255 & +0.00353913 & $-9.42854 \mathrm{E}-05$ \\
6 & 0.983657 & -1.05261 & +0.373706 & -0.0611939 & +0.00432395 & -0.000111318 \\
7 & 1.43123 & -1.37401 & +0.457479 & -0.0701771 & +0.00476888 & -0.000119736 \\
8 & 1.77715 & -1.59855 & +0.508672 & -0.0746767 & +0.0049316 & -0.000121413 \\
9 & 1.95518 & -1.68507 & +0.518422 & -0.0736588 & +0.00475164 & -0.00011497 \\
10 & 1.94147 & -1.62519 & +0.487084 & -0.0674302 & +0.00425874 & -0.000101277 \\
11 & 1.75802 & -1.44491 & +0.424434 & -0.0575055 & +0.00355913 & $-8.31272 \mathrm{E}-05$ \\
12 & 1.4799 & -1.20209 & +0.346908 & -0.0460367 & +0.0027865 & $-6.37114 \mathrm{E}-05$ \\
13 & 1.22284 & -0.97796 & +0.275905 & -0.0356545 & +0.0020937 & $-4.64261 \mathrm{E}-05$ \\
14 & 0.952053 & -0.750586 & +0.206158 & -0.0257512 & +0.0014481 & $-3.06167 \mathrm{E}-05$ \\
15 & 0.658001 & -0.516408 & +0.137404 & -0.0163519 & +0.000853571 & $-1.64193 \mathrm{E}-05$ \\
\hline
\end{tabular}


Table 6.54: Fit result of Eq. (6.7) to different apertures and models, for the correction to infinite aperture, as a function of the measured size of a cluster. NICMOS/NIC2 chip, F110W filter, pre-cooler

\begin{tabular}{|c|c|c|c|c|c|c|}
\hline \multicolumn{7}{|c|}{ King 30 } \\
\hline Aperture (pixels) & $a$ & $b$ & $c$ & $d$ & $e$ & $f$ \\
\hline 2 & -6.19635 & +5.65025 & -1.92406 & +0.271275 & -0.0177214 & +0.000442102 \\
3 & -8.68818 & +7.33586 & -2.29456 & +0.313468 & -0.0201534 & +0.000498148 \\
4 & -9.41298 & +7.7241 & -2.33801 & +0.315293 & -0.0201391 & +0.000495953 \\
5 & -9.93905 & +7.94642 & -2.34949 & +0.313989 & -0.0199691 & +0.000490626 \\
6 & -10.0223 & +7.8599 & -2.28725 & +0.303629 & -0.0192397 & +0.000471592 \\
7 & -9.82108 & +7.58394 & -2.17969 & +0.287644 & -0.0181593 & +0.000443903 \\
8 & -9.4063 & +7.18328 & -2.04603 & +0.268725 & -0.0169085 & +0.000412217 \\
9 & -8.82408 & +6.67912 & -1.88855 & +0.246977 & -0.0154903 & +0.000376632 \\
10 & -8.16976 & +6.13955 & -1.72522 & +0.224667 & -0.0140431 & +0.000340451 \\
11 & -7.4827 & +5.588 & -1.56112 & +0.202351 & -0.0125957 & +0.000304191 \\
12 & -6.89792 & +5.12369 & -1.42452 & +0.183999 & -0.0114196 & +0.000275075 \\
13 & -6.23441 & +4.60716 & -1.27431 & +0.16381 & -0.0101217 & +0.000242828 \\
14 & -5.68218 & +4.17802 & -1.14987 & +0.147156 & -0.00905474 & +0.000216376 \\
15 & -5.14828 & +3.76714 & -1.03174 & +0.131429 & -0.00805208 & +0.00019164 \\
\hline
\end{tabular}


Table 6.55: Fit result of Eq. (6.7) to different apertures and models, for the correction to infinite aperture, as a function of the measured size of a cluster. NICMOS/NIC2 chip, F110W filter, pre-cooler

\begin{tabular}{|c|c|c|c|c|c|c|}
\hline \multicolumn{7}{|c|}{ EFF 15 } \\
\hline Aperture (pixels) & $a$ & $b$ & $c$ & $d$ & $e$ & $f$ \\
\hline 2 & 5.39906 & -3.29231 & +0.640393 & -0.0725786 & +0.00422967 & $-9.76321 \mathrm{E}-05$ \\
3 & 2.65887 & -1.43763 & +0.229069 & -0.0260708 & +0.00160393 & $-3.89713 \mathrm{E}-05$ \\
4 & 1.9497 & -1.02566 & +0.171373 & -0.0216965 & +0.00142862 & $-3.60554 \mathrm{E}-05$ \\
5 & 1.41258 & -0.740924 & +0.130262 & -0.0179615 & +0.00123103 & $-3.15842 \mathrm{E}-05$ \\
6 & 1.05635 & -0.564594 & +0.104696 & -0.0152502 & +0.00106113 & $-2.72357 \mathrm{E}-05$ \\
7 & 0.845925 & -0.47449 & +0.0954762 & -0.0143042 & +0.000995196 & $-2.54193 \mathrm{E}-05$ \\
8 & 0.712204 & -0.413386 & +0.0866729 & -0.01295 & +0.000887312 & $-2.23196 \mathrm{E}-05$ \\
9 & 0.603766 & -0.360751 & +0.0779162 & -0.0116053 & +0.000786688 & $-1.96004 \mathrm{E}-05$ \\
10 & 0.571461 & -0.351992 & +0.0786085 & -0.0114127 & +0.000757184 & $-1.85929 \mathrm{E}-05$ \\
11 & 0.475999 & -0.294704 & +0.0656954 & -0.00957681 & +0.000634973 & $-1.55809 \mathrm{E}-05$ \\
12 & 0.440184 & -0.274791 & +0.0615552 & -0.0087836 & +0.000571433 & $-1.38003 \mathrm{E}-05$ \\
13 & 0.392465 & -0.247027 & +0.0557735 & -0.00794402 & +0.00051662 & $-1.25137 \mathrm{E}-05$ \\
14 & 0.33693 & -0.211582 & +0.0472769 & -0.00670542 & +0.000433157 & $-1.04183 \mathrm{E}-05$ \\
15 & 0.290945 & -0.182 & +0.0402679 & -0.0057094 & +0.000368229 & $-8.84698 \mathrm{E}-06$ \\
\hline
\end{tabular}


Table 6.56: Fit result of Eq. (6.7) to different apertures and models, for the correction to infinite aperture, as a function of the measured size of a cluster. NICMOS/NIC2 chip, F110W filter, post-cooler

\begin{tabular}{|c|c|c|c|c|c|c|}
\hline \multicolumn{7}{|c|}{ King 5 } \\
\hline Aperture (pixels) & $a$ & $b$ & $c$ & $d$ & $e$ & $f$ \\
\hline 2 & -0.735602 & +0.319557 & -0.114792 & +0.000632984 & +0.000980036 & $-4.74102 \mathrm{E}-05$ \\
3 & -0.421244 & +0.0993929 & +0.015535 & -0.0212032 & +0.00253726 & $-8.83765 \mathrm{E}-05$ \\
4 & -0.120788 & -0.190403 & +0.138287 & -0.0388386 & +0.00365122 & -0.000114691 \\
5 & 0.0544896 & -0.365195 & +0.202367 & -0.0460347 & +0.00396817 & -0.000118755 \\
6 & 0.153227 & -0.43645 & +0.218401 & -0.0453418 & +0.00372097 & -0.000107902 \\
7 & 0.180232 & -0.420718 & +0.199414 & -0.0392415 & +0.00310167 & $-8.74724 \mathrm{E}-05$ \\
8 & 0.176455 & -0.377177 & +0.1717 & -0.0324113 & +0.00247484 & $-6.78795 \mathrm{E}-05$ \\
9 & 0.148687 & -0.312634 & +0.137609 & -0.0249928 & +0.00183429 & $-4.85721 \mathrm{E}-05$ \\
10 & 0.117974 & -0.251913 & +0.107065 & -0.0186415 & +0.00130252 & $-3.29106 \mathrm{E}-05$ \\
11 & 0.0792552 & -0.186603 & +0.0767279 & -0.0127332 & +0.00082754 & $-1.93092 \mathrm{E}-05$ \\
12 & 0.0417971 & -0.126239 & +0.0494624 & -0.00757946 & +0.000422485 & $-7.90776 \mathrm{E}-06$ \\
13 & 0.0165576 & -0.0838937 & +0.0305825 & -0.00410109 & +0.000159977 & $-8.5189 \mathrm{E}-07$ \\
14 & -0.0100506 & -0.0426289 & +0.0130249 & -0.000994945 & $-6.76685 \mathrm{E}-05$ & $+5.13434 \mathrm{E}-06$ \\
15 & -0.0273418 & -0.0149416 & +0.00150664 & +0.000974519 & -0.000204309 & $+8.50458 \mathrm{E}-06$ \\
\hline
\end{tabular}


Table 6.57: Fit result of Eq. (6.7) to different apertures and models, for the correction to infinite aperture, as a function of the measured size of a cluster. NICMOS/NIC2 chip, F110W filter, post-cooler

\begin{tabular}{|c|c|c|c|c|c|c|}
\hline \multicolumn{7}{|c|}{ King 30 } \\
\hline Aperture (pixels) & $a$ & $b$ & $c$ & $d$ & $e$ & $f$ \\
\hline 2 & -2.45178 & +2.38149 & -0.947794 & +0.135573 & -0.00871294 & +0.000211072 \\
3 & -2.691 & +2.63663 & -0.967001 & +0.136041 & -0.00874514 & +0.000213583 \\
4 & -2.71734 & +2.61902 & -0.92052 & +0.128484 & -0.00827793 & +0.000203556 \\
5 & -2.69163 & +2.53198 & -0.863568 & +0.119803 & -0.00772307 & +0.000190502 \\
6 & -2.52482 & +2.34079 & -0.784124 & +0.108113 & -0.00694904 & +0.000171076 \\
7 & -2.31349 & +2.12247 & -0.700696 & +0.0957987 & -0.00611194 & +0.000149303 \\
8 & -2.10322 & +1.91256 & -0.623806 & +0.0845231 & -0.00534524 & +0.000129348 \\
9 & -1.88434 & +1.69476 & -0.545039 & +0.0728071 & -0.00453289 & +0.000107778 \\
10 & -1.68622 & +1.50159 & -0.476988 & +0.0628627 & -0.00385476 & $+9.00767 \mathrm{E}-05$ \\
11 & -1.49499 & +1.31668 & -0.412293 & +0.0533184 & -0.00319512 & $+7.26069 \mathrm{E}-05$ \\
12 & -1.32 & +1.14937 & -0.35447 & +0.0448558 & -0.00261432 & $+5.73267 \mathrm{E}-05$ \\
13 & -1.15596 & +0.99485 & -0.302061 & +0.0372916 & -0.00210291 & $+4.4097 \mathrm{E}-05$ \\
14 & -1.0166 & +0.864884 & -0.258348 & +0.0310545 & -0.00168506 & $+3.33503 \mathrm{E}-05$ \\
15 & -0.894502 & +0.750923 & -0.220197 & +0.0256473 & -0.00132666 & $+2.42712 \mathrm{E}-05$ \\
\hline
\end{tabular}


Table 6.58: Fit result of Eq. (6.7) to different apertures and models, for the correction to infinite aperture, as a function of the measured size of a cluster. NICMOS/NIC2 chip, F110W filter, post-cooler

\begin{tabular}{|c|c|c|c|c|c|c|}
\hline \multicolumn{7}{|c|}{ EFF 15 } \\
\hline Aperture (pixels) & $a$ & $b$ & $c$ & $d$ & $e$ & $f$ \\
\hline 2 & -1.08061 & +0.841751 & -0.393803 & +0.0525897 & -0.00310015 & $+6.9007 \mathrm{E}-05$ \\
3 & -0.877458 & +0.730017 & -0.298842 & +0.0361328 & -0.00193253 & $+3.88448 \mathrm{E}-05$ \\
4 & -0.640728 & +0.512045 & -0.196392 & +0.0209741 & -0.00095848 & $+1.55282 \mathrm{E}-05$ \\
5 & -0.491248 & +0.356838 & -0.129055 & +0.0118599 & -0.000422378 & $+3.79131 \mathrm{E}-06$ \\
6 & -0.371275 & +0.247502 & -0.0872785 & +0.00686376 & -0.000164971 & $-1.05164 \mathrm{E}-06$ \\
7 & -0.293047 & +0.187961 & -0.0663502 & +0.00497879 & -0.000112174 & $-8.61339 \mathrm{E}-07$ \\
8 & -0.238101 & +0.147501 & -0.0521959 & +0.00373339 & $-7.54624 \mathrm{E}-05$ & $-8.98696 \mathrm{E}-07$ \\
9 & -0.20329 & +0.12436 & -0.0442984 & +0.00329889 & $-8.60427 \mathrm{E}-05$ & $+1.16511 \mathrm{E}-07$ \\
10 & -0.178822 & +0.109233 & -0.0391943 & +0.00311642 & -0.000103553 & $+1.10204 \mathrm{E}-06$ \\
11 & -0.149455 & +0.0876909 & -0.0316751 & +0.00239644 & $-7.30088 \mathrm{E}-05$ & $+6.16027 \mathrm{E}-07$ \\
12 & -0.13853 & +0.0835702 & -0.0302361 & +0.00253805 & $-9.89349 \mathrm{E}-05$ & $+1.53412 \mathrm{E}-06$ \\
13 & -0.118868 & +0.0701629 & -0.0255853 & +0.0021113 & $-8.09812 \mathrm{E}-05$ & $+1.23364 \mathrm{E}-06$ \\
14 & -0.108319 & +0.0650233 & -0.0238699 & +0.00211199 & $-9.24538 \mathrm{E}-05$ & $+1.71206 \mathrm{E}-06$ \\
15 & -0.0987899 & +0.0591169 & -0.0215729 & +0.00193677 & $-8.69028 \mathrm{E}-05$ & $+1.65223 \mathrm{E}-06$ \\
\hline
\end{tabular}


Table 6.59: Fit result of Eq. (6.7) to different apertures and models, for the correction to infinite aperture, as a function of the measured size of a given cluster. NICMOS/NIC2 chip, F160W filter, pre-cooler

\begin{tabular}{|c|c|c|c|c|c|c|}
\hline \multicolumn{7}{|c|}{ King 5 } \\
\hline Aperture (pixels) & $a$ & $b$ & $c$ & $d$ & $e$ & $f$ \\
\hline 2 & -1.09707 & +0.0755088 & +0.0294664 & -0.022103 & +0.00238031 & $-7.61415 \mathrm{E}-05$ \\
3 & -0.557716 & -0.174551 & +0.133531 & -0.0364039 & +0.00323297 & $-9.49878 \mathrm{E}-05$ \\
4 & -0.167724 & -0.360539 & +0.19303 & -0.042334 & +0.0034497 & $-9.66062 \mathrm{E}-05$ \\
5 & 0.0324483 & -0.417252 & +0.200673 & -0.0402498 & +0.0031217 & $-8.45416 \mathrm{E}-05$ \\
6 & 0.110924 & -0.419075 & +0.190475 & -0.0359825 & +0.00267791 & $-7.02494 \mathrm{E}-05$ \\
7 & 0.139966 & -0.392018 & +0.169747 & -0.0304228 & +0.00216926 & $-5.48646 \mathrm{E}-05$ \\
8 & 0.125724 & -0.333877 & +0.139151 & -0.0238141 & +0.0016165 & $-3.89256 \mathrm{E}-05$ \\
9 & 0.0907749 & -0.25917 & +0.103802 & -0.0168545 & +0.00106419 & $-2.35453 \mathrm{E}-05$ \\
10 & 0.0491138 & -0.185538 & +0.0710413 & -0.0107463 & +0.000596853 & $-1.08773 \mathrm{E}-05$ \\
11 & 0.00934512 & -0.12019 & +0.0429004 & -0.00568585 & +0.000221498 & $-9.72959 \mathrm{E}-07$ \\
12 & -0.0274029 & -0.0643577 & +0.0196798 & -0.00163404 & $-7.09162 \mathrm{E}-05$ & $+6.55733 \mathrm{E}-06$ \\
13 & -0.0557109 & -0.0208479 & +0.00204259 & +0.0013295 & -0.000274926 & $+1.15442 \mathrm{E}-05$ \\
14 & -0.0774913 & +0.0128172 & -0.0112068 & +0.00347709 & -0.000415756 & $+1.48008 \mathrm{E}-05$ \\
15 & -0.0928398 & +0.0385324 & -0.0210262 & +0.00498535 & -0.000507086 & $+1.66966 \mathrm{E}-05$ \\
\hline
\end{tabular}


Table 6.60: Fit result of Eq. (6.7) to different apertures and models, for the correction to infinite aperture, as a function of the measured size of a given cluster. NICMOS/NIC2 chip, F160W filter, pre-cooler

\begin{tabular}{|c|c|c|c|c|c|c|}
\hline \multicolumn{7}{|c|}{ King 30 } \\
\hline Aperture (pixels) & $a$ & $b$ & $c$ & $d$ & $e$ & $f$ \\
\hline 2 & -7.47571 & +5.5141 & -1.63352 & +0.203639 & -0.0117814 & +0.000260208 \\
3 & -7.12485 & +5.34356 & -1.53915 & +0.189569 & -0.0109036 & +0.000240218 \\
4 & -6.5043 & +4.92779 & -1.39894 & +0.171292 & -0.0098324 & +0.000216623 \\
5 & -5.89815 & +4.49129 & -1.26242 & +0.15395 & -0.00882282 & +0.00019429 \\
6 & -5.40528 & +4.09775 & -1.13922 & +0.138116 & -0.00788444 & +0.000173077 \\
7 & -4.88726 & +3.67602 & -1.01056 & +0.121516 & -0.00688571 & +0.000150021 \\
8 & -4.36358 & +3.252 & -0.883702 & +0.105065 & -0.00588301 & +0.000126508 \\
9 & -3.84676 & +2.83861 & -0.761801 & +0.0892749 & -0.00491677 & +0.000103705 \\
10 & -3.36681 & +2.45893 & -0.651308 & +0.0750345 & -0.00404782 & $+8.32487 \mathrm{E}-05$ \\
11 & -2.94422 & +2.12781 & -0.556088 & +0.0628793 & -0.00331267 & $+6.60931 \mathrm{E}-05$ \\
12 & -2.55706 & +1.82339 & -0.46841 & +0.051606 & -0.00262382 & $+4.98232 \mathrm{E}-05$ \\
13 & -2.2207 & +1.56249 & -0.394474 & +0.0422801 & -0.0020666 & $+3.69933 \mathrm{E}-05$ \\
14 & -1.9295 & +1.33947 & -0.332194 & +0.0345646 & -0.00161414 & $+2.67687 \mathrm{E}-05$ \\
15 & -1.63946 & +1.11764 & -0.269945 & +0.0267184 & -0.0011437 & $+1.587 \mathrm{E}-05$ \\
\hline
\end{tabular}


Table 6.61: Fit result of Eq. (6.7) to different apertures and models, for the correction to infinite aperture, as a function of the measured size of a given cluster. NICMOS/NIC2 chip, F160W filter, pre-cooler

\begin{tabular}{|c|c|c|c|c|c|c|}
\hline \multicolumn{7}{|c|}{ EFF 15 } \\
\hline Aperture (pixels) & $a$ & $b$ & $c$ & $d$ & $e$ & $f$ \\
\hline 2 & -2.72017 & +1.6075 & -0.498158 & +0.0549803 & -0.00269103 & $+4.84718 \mathrm{E}-05$ \\
3 & -2.02657 & +1.22991 & -0.355302 & +0.0353141 & -0.00149428 & $+2.12957 \mathrm{E}-05$ \\
4 & -1.45485 & +0.894716 & -0.247431 & +0.0221982 & -0.000785591 & $+7.06514 \mathrm{E}-06$ \\
5 & -1.07373 & +0.672139 & -0.181115 & +0.0150584 & -0.000457138 & $+1.81762 \mathrm{E}-06$ \\
6 & -0.855698 & +0.532819 & -0.139831 & +0.0109848 & -0.000297326 & $+2.69539 \mathrm{E}-08$ \\
7 & -0.684408 & +0.416716 & -0.106883 & +0.00790847 & -0.000186922 & $-9.15832 \mathrm{E}-07$ \\
8 & -0.564417 & +0.336046 & -0.0851491 & +0.00611656 & -0.000139904 & $-7.33571 \mathrm{E}-07$ \\
9 & -0.481221 & +0.28492 & -0.0726802 & +0.0054389 & -0.00015187 & $+5.89555 \mathrm{E}-07$ \\
10 & -0.41836 & +0.247392 & -0.0636623 & +0.00497321 & -0.00016109 & $+1.50714 \mathrm{E}-06$ \\
11 & -0.363253 & +0.213242 & -0.0553274 & +0.00445381 & -0.000158386 & $+1.97532 \mathrm{E}-06$ \\
12 & -0.318082 & +0.18407 & -0.0477641 & +0.00387272 & -0.000141987 & $+1.90733 \mathrm{E}-06$ \\
13 & -0.283183 & +0.163318 & -0.0428876 & +0.00362328 & -0.000145556 & $+2.32768 \mathrm{E}-06$ \\
14 & -0.257515 & +0.148888 & -0.0395695 & +0.00350609 & -0.000153026 & $+2.74555 \mathrm{E}-06$ \\
15 & -0.230931 & +0.132739 & -0.0353947 & +0.00317858 & -0.000142148 & $+2.63446 \mathrm{E}-06$ \\
\hline
\end{tabular}


Table 6.62: Fit result of Eq. (6.7) to different apertures and models, for the correction to infinite aperture, as a function of the measured size of a given cluster. NICMOS/NIC2 chip, F160W filter, post-cooler

\begin{tabular}{|c|c|c|c|c|c|c|}
\hline \multicolumn{7}{|c|}{ King 5 } \\
\hline Aperture (pixels) & $a$ & $b$ & $c$ & $d$ & $e$ & $f$ \\
\hline 2 & -1.05907 & +0.317635 & -0.0708061 & -0.00772923 & +0.00150457 & $-5.68309 \mathrm{E}-05$ \\
3 & -0.56267 & +0.00796501 & +0.0687276 & -0.0286155 & +0.0028662 & $-8.98268 \mathrm{E}-05$ \\
4 & -0.140716 & -0.258877 & +0.159934 & -0.0394265 & +0.00341421 & $-9.9732 \mathrm{E}-05$ \\
5 & 0.0547544 & -0.377886 & +0.194346 & -0.0414785 & +0.00336145 & $-9.4343 \mathrm{E}-05$ \\
6 & 0.137591 & -0.430326 & +0.203724 & -0.0399704 & +0.00308349 & $-8.36152 \mathrm{E}-05$ \\
7 & 0.174214 & -0.434084 & +0.193294 & -0.0356283 & +0.00263054 & $-6.89217 \mathrm{E}-05$ \\
8 & 0.156075 & -0.37847 & +0.162271 & -0.0286168 & +0.00202461 & $-5.09678 \mathrm{E}-05$ \\
9 & 0.134214 & -0.31325 & +0.128635 & -0.0216528 & +0.00145361 & $-3.46478 \mathrm{E}-05$ \\
10 & 0.0896687 & -0.229598 & +0.0905932 & -0.0144572 & +0.000891437 & $-1.90467 \mathrm{E}-05$ \\
11 & 0.049704 & -0.162052 & +0.0610629 & -0.00904396 & +0.000480981 & $-7.96621 \mathrm{E}-06$ \\
12 & 0.0145889 & -0.10657 & +0.0371294 & -0.00472132 & +0.000158774 & $+5.8799 \mathrm{E}-07$ \\
13 & -0.019128 & -0.0590899 & +0.0178245 & -0.00139722 & $-7.81879 \mathrm{E}-05$ & $+6.61258 \mathrm{E}-06$ \\
14 & -0.0435607 & -0.0216875 & +0.00275374 & +0.00112144 & -0.000250249 & $+1.07817 \mathrm{E}-05$ \\
15 & -0.0630181 & +0.00933645 & -0.00947897 & +0.00310562 & -0.000381596 & $+1.38639 \mathrm{E}-05$ \\
\hline
\end{tabular}


Table 6.63: Fit result of Eq. (6.7) to different apertures and models, for the correction to infinite aperture, as a function of the measured size of a given cluster. NICMOS/NIC2 chip, F160W filter, post-cooler

\begin{tabular}{|c|c|c|c|c|c|c|}
\hline \multicolumn{7}{|c|}{ King 30 } \\
\hline Aperture (pixels) & $a$ & $b$ & $c$ & $d$ & $e$ & $f$ \\
\hline 2 & -5.54481 & +4.51936 & -1.46585 & +0.19359 & -0.0117878 & +0.000273664 \\
3 & -5.3748 & +4.44115 & -1.38928 & +0.181173 & -0.0109866 & +0.000255164 \\
4 & -4.8493 & +4.05671 & -1.24995 & +0.162211 & -0.00983158 & +0.000228725 \\
5 & -4.48351 & +3.73956 & -1.13575 & +0.146777 & -0.00889259 & +0.000207143 \\
6 & -4.15447 & +3.41711 & -1.0221 & +0.131126 & -0.0079083 & +0.000183587 \\
7 & -3.76386 & +3.05272 & -0.9008 & +0.114515 & -0.00685326 & +0.000157917 \\
8 & -3.36969 & +2.70052 & -0.78691 & +0.0988846 & -0.00584888 & +0.000133119 \\
9 & -2.95784 & +2.35148 & -0.677918 & +0.0841242 & -0.004906 & +0.000109893 \\
10 & -2.58307 & +2.03919 & -0.581486 & +0.0710843 & -0.00407224 & $+8.93393 \mathrm{E}-05$ \\
11 & -2.26378 & +1.77015 & -0.498159 & +0.0597621 & -0.00334361 & $+7.124 \mathrm{E}-05$ \\
12 & -1.98295 & +1.53253 & -0.4251 & +0.0499495 & -0.00272173 & $+5.60728 \mathrm{E}-05$ \\
13 & -1.7468 & +1.33192 & -0.363368 & +0.0416512 & -0.00219464 & $+4.31744 \mathrm{E}-05$ \\
14 & -1.5235 & +1.14646 & -0.307422 & +0.0342579 & -0.00173268 & $+3.2048 \mathrm{E}-05$ \\
15 & -1.31427 & +0.974076 & -0.255577 & +0.0273787 & -0.00130033 & $+2.15691 \mathrm{E}-05$ \\
\hline
\end{tabular}


Table 6.64: Fit result of Eq. (6.7) to different apertures and models, for the correction to infinite aperture, as a function of the measured size of a given cluster. NICMOS/NIC2 chip, F160W filter, post-cooler

\begin{tabular}{|c|c|c|c|c|c|c|}
\hline \multicolumn{7}{|c|}{ EFF 15 } \\
\hline Aperture (pixels) & $a$ & $b$ & $c$ & $d$ & $e$ & $f$ \\
\hline 2 & -2.19182 & +1.47713 & -0.508301 & +0.0599628 & -0.00313174 & $+6.05755 \mathrm{E}-05$ \\
3 & -1.64242 & +1.12849 & -0.357302 & +0.0375692 & -0.00168073 & $+2.56276 \mathrm{E}-05$ \\
4 & -1.11122 & +0.776543 & -0.23691 & +0.0221503 & -0.000801261 & $+6.88721 \mathrm{E}-06$ \\
5 & -0.824877 & +0.568754 & -0.166545 & +0.0138983 & -0.000384587 & $-6.59241 \mathrm{E}-07$ \\
6 & -0.661523 & +0.429087 & -0.119638 & +0.00874939 & -0.000150254 & $-4.20981 \mathrm{E}-06$ \\
7 & -0.52711 & +0.315488 & -0.0836856 & +0.00500706 & $+1.10635 \mathrm{E}-05$ & $-6.47949 \mathrm{E}-06$ \\
8 & -0.445138 & +0.256271 & -0.0671247 & +0.0038891 & $+1.21735 \mathrm{E}-05$ & $-5.04061 \mathrm{E}-06$ \\
9 & -0.369984 & +0.2102 & -0.0555056 & +0.00328106 & $-4.27563 \mathrm{E}-06$ & $-3.54775 \mathrm{E}-06$ \\
10 & -0.318111 & +0.183594 & -0.049568 & +0.00322741 & $-4.09528 \mathrm{E}-05$ & $-1.89881 \mathrm{E}-06$ \\
11 & -0.277707 & +0.161126 & -0.0441867 & +0.00307645 & $-6.17961 \mathrm{E}-05$ & $-8.22911 \mathrm{E}-07$ \\
12 & -0.251616 & +0.146131 & -0.0403395 & +0.00298707 & $-7.83161 \mathrm{E}-05$ & $-1.67083 \mathrm{E}-08$ \\
13 & -0.226254 & +0.129262 & -0.0354883 & +0.00263862 & $-7.13726 \mathrm{E}-05$ & $+8.33658 \mathrm{E}-08$ \\
14 & -0.200333 & +0.113288 & -0.0312326 & +0.00236613 & $-6.92614 \mathrm{E}-05$ & $+3.16587 \mathrm{E}-07$ \\
15 & -0.181721 & +0.103717 & -0.0289866 & +0.00233036 & $-7.9897 \mathrm{E}-05$ & $+8.02955 \mathrm{E}-07$ \\
\hline
\end{tabular}


Table 6.65: Fit result of Eq. (6.7) to different apertures and models, for the correction to infinite aperture, as a function of the measured size of a given cluster. NICMOS/NIC2 chip, F205W filter, pre-cooler

\begin{tabular}{|c|c|c|c|c|c|c|}
\hline \multicolumn{7}{|c|}{ King 5 } \\
\hline Aperture (pixels) & $a$ & $b$ & $c$ & $d$ & $e$ & $f$ \\
\hline 2 & -1.09459 & +0.0794794 & -0.014953 & -0.00946316 & +0.00118036 & $-3.85004 \mathrm{E}-05$ \\
3 & -0.652646 & -0.119652 & +0.0857263 & -0.0248698 & +0.00219581 & $-6.33195 \mathrm{E}-05$ \\
4 & -0.253843 & -0.344405 & +0.169305 & -0.0357675 & +0.00282876 & $-7.7202 \mathrm{E}-05$ \\
5 & 0.0392393 & -0.455766 & +0.197067 & -0.0373448 & +0.00279772 & $-7.38198 \mathrm{E}-05$ \\
6 & 0.154276 & -0.463056 & +0.191134 & -0.0343795 & +0.00248764 & $-6.39674 \mathrm{E}-05$ \\
7 & 0.192853 & -0.443411 & +0.177916 & -0.0306863 & +0.00214967 & $-5.38572 \mathrm{E}-05$ \\
8 & 0.198932 & -0.409379 & +0.159152 & -0.0263473 & +0.00178067 & $-4.32283 \mathrm{E}-05$ \\
9 & 0.177533 & -0.354965 & +0.133632 & -0.0212194 & +0.00137303 & $-3.19411 \mathrm{E}-05$ \\
10 & 0.130692 & -0.281614 & +0.102898 & -0.0156403 & +0.000954023 & $-2.0762 \mathrm{E}-05$ \\
11 & 0.0841123 & -0.211562 & +0.074426 & -0.0106775 & +0.000592588 & $-1.13447 \mathrm{E}-05$ \\
12 & 0.0369902 & -0.143467 & +0.04753 & -0.00614973 & +0.00027142 & $-3.14806 \mathrm{E}-06$ \\
13 & -0.00343348 & -0.0874627 & +0.026378 & -0.00275005 & $+4.17426 \mathrm{E}-05$ & $+2.4291 \mathrm{E}-06$ \\
14 & -0.0327284 & -0.0441706 & +0.00984919 & -0.00010892 & -0.000133723 & $+6.6051 \mathrm{E}-06$ \\
15 & -0.0594866 & -0.00908039 & -0.00260292 & +0.00174909 & -0.000247652 & $+9.07164 \mathrm{E}-06$ \\
\hline
\end{tabular}


Table 6.66: Fit result of Eq. (6.7) to different apertures and models, for the correction to infinite aperture, as a function of the measured size of a given cluster. NICMOS/NIC2 chip, F205W filter, pre-cooler

\begin{tabular}{|c|c|c|c|c|c|c|}
\hline \multicolumn{7}{|c|}{ King 30 } \\
\hline Aperture (pixels) & $a$ & $b$ & $c$ & $d$ & $e$ & $f$ \\
\hline 2 & -2.35261 & +1.32102 & -0.423556 & +0.0415269 & -0.00146961 & $+8.10503 \mathrm{E}-06$ \\
3 & -2.34297 & +1.49781 & -0.441535 & +0.0436512 & -0.00167605 & $+1.56361 \mathrm{E}-05$ \\
4 & -2.22948 & +1.51091 & -0.427148 & +0.042377 & -0.00169055 & $+1.86102 \mathrm{E}-05$ \\
5 & -1.9463 & +1.38298 & -0.384054 & +0.0377092 & -0.00148465 & $+1.57115 \mathrm{E}-05$ \\
6 & -1.77126 & +1.29042 & -0.352168 & +0.0343538 & -0.00134555 & $+1.4057 \mathrm{E}-05$ \\
7 & -1.6314 & +1.1868 & -0.315468 & +0.0300047 & -0.00112011 & $+9.76408 \mathrm{E}-06$ \\
8 & -1.47429 & +1.05661 & -0.272502 & +0.0247377 & -0.000827461 & $+3.60366 \mathrm{E}-06$ \\
9 & -1.29949 & +0.908462 & -0.225171 & +0.0188119 & -0.000485332 & $-3.95232 \mathrm{E}-06$ \\
10 & -1.13549 & +0.772032 & -0.183216 & +0.013685 & -0.000196583 & $-1.01437 \mathrm{E}-05$ \\
11 & -0.976181 & +0.642021 & -0.143952 & +0.00884759 & $+8.15574 \mathrm{E}-05$ & $-1.62927 \mathrm{E}-05$ \\
12 & -0.826777 & +0.52376 & -0.109416 & +0.00472501 & +0.000309943 & $-2.1116 \mathrm{E}-05$ \\
13 & -0.701667 & +0.425908 & -0.0812112 & +0.00142435 & +0.000488178 & $-2.47548 \mathrm{E}-05$ \\
14 & -0.576057 & +0.327276 & -0.0529574 & -0.00190788 & +0.000670331 & $-2.8536 \mathrm{E}-05$ \\
15 & -0.477245 & +0.250537 & -0.0314897 & -0.00432573 & +0.000794395 & $-3.0896 \mathrm{E}-05$ \\
\hline
\end{tabular}


Table 6.67: Fit result of Eq. (6.7) to different apertures and models, for the correction to infinite aperture, as a function of the measured size of a given cluster. NICMOS/NIC2 chip, F205W filter, pre-cooler

\begin{tabular}{|c|c|c|c|c|c|c|}
\hline \multicolumn{7}{|c|}{ EFF 15 } \\
\hline Aperture (pixels) & $a$ & $b$ & $c$ & $d$ & $e$ & $f$ \\
\hline 2 & -1.15821 & +0.231947 & -0.115808 & +0.00739641 & $+7.39942 \mathrm{E}-05$ & $-1.27437 \mathrm{E}-05$ \\
3 & -0.84275 & +0.168615 & -0.0610412 & -0.00107245 & +0.000604755 & $-2.48392 \mathrm{E}-05$ \\
4 & -0.573214 & +0.0729277 & -0.0170542 & -0.0064596 & +0.000876212 & $-2.96667 \mathrm{E}-05$ \\
5 & -0.316857 & -0.0122418 & +0.00887833 & -0.00852508 & +0.000913199 & $-2.8615 \mathrm{E}-05$ \\
6 & -0.212333 & -0.0246151 & +0.0126424 & -0.0078538 & +0.000798499 & $-2.44287 \mathrm{E}-05$ \\
7 & -0.162494 & -0.0313319 & +0.016109 & -0.00744025 & +0.000718391 & $-2.1452 \mathrm{E}-05$ \\
8 & -0.141369 & -0.0284354 & +0.0154075 & -0.00652141 & +0.000611955 & $-1.79547 \mathrm{E}-05$ \\
9 & -0.11623 & -0.0340087 & +0.0166135 & -0.00595697 & +0.000536531 & $-1.54119 \mathrm{E}-05$ \\
10 & -0.103608 & -0.0317767 & +0.0153508 & -0.00521684 & +0.000460943 & $-1.31008 \mathrm{E}-05$ \\
11 & -0.0890165 & -0.0323279 & +0.0148816 & -0.00469931 & +0.00040642 & $-1.1427 \mathrm{E}-05$ \\
12 & -0.084616 & -0.0230453 & +0.0108789 & -0.00370723 & +0.000322335 & $-9.04368 \mathrm{E}-06$ \\
13 & -0.0714046 & -0.0232235 & +0.0100352 & -0.00325229 & +0.000278424 & $-7.7474 \mathrm{E}-06$ \\
14 & -0.0657639 & -0.0190475 & +0.00814515 & -0.00271975 & +0.000232711 & $-6.45643 \mathrm{E}-06$ \\
15 & -0.0646294 & -0.0136388 & +0.00619566 & -0.00224815 & +0.000194435 & $-5.41561 \mathrm{E}-06$ \\
\hline
\end{tabular}


Table 6.68: Fit result of Eq. (6.7) to different apertures and models, for the correction to infinite aperture, as a function of the measured size of a given cluster. NICMOS/NIC2 chip, F205W filter, post-cooler

\begin{tabular}{|c|c|c|c|c|c|c|}
\hline \multicolumn{7}{|c|}{ King 5 } \\
\hline Aperture (pixels) & $a$ & $b$ & $c$ & $d$ & $e$ & $f$ \\
\hline 2 & -1.00242 & +0.146307 & -0.0265553 & -0.0105957 & +0.00143694 & $-4.88893 \mathrm{E}-05$ \\
3 & -0.572564 & -0.118236 & +0.102064 & -0.0303374 & +0.00274781 & $-8.11881 \mathrm{E}-05$ \\
4 & -0.141862 & -0.388412 & +0.197496 & -0.0424083 & +0.00342594 & $-9.54935 \mathrm{E}-05$ \\
5 & 0.139998 & -0.497554 & +0.220896 & -0.0427356 & +0.00327316 & $-8.8193 \mathrm{E}-05$ \\
6 & 0.221196 & -0.500022 & +0.21286 & -0.0391214 & +0.00289426 & $-7.60005 \mathrm{E}-05$ \\
7 & 0.24345 & -0.485623 & +0.199889 & -0.0350505 & +0.00250295 & $-6.38977 \mathrm{E}-05$ \\
8 & 0.237933 & -0.450345 & +0.178862 & -0.0300286 & +0.00206633 & $-5.10852 \mathrm{E}-05$ \\
9 & 0.198046 & -0.381976 & +0.14658 & -0.0235449 & +0.00154476 & $-3.64097 \mathrm{E}-05$ \\
10 & 0.14557 & -0.301167 & +0.111481 & -0.0170544 & +0.00104819 & $-2.29093 \mathrm{E}-05$ \\
11 & 0.0900989 & -0.219704 & +0.0781546 & -0.011244 & +0.000622193 & $-1.17066 \mathrm{E}-05$ \\
12 & 0.0412742 & -0.144891 & +0.048034 & -0.0061413 & +0.000257464 & $-2.32177 \mathrm{E}-06$ \\
13 & -0.00157967 & -0.0820638 & +0.0238291 & -0.0022064 & $-1.30111 \mathrm{E}-05$ & $+4.38603 \mathrm{E}-06$ \\
14 & -0.0339072 & -0.0369037 & +0.00714583 & +0.000387692 & -0.000180118 & $+8.2076 \mathrm{E}-06$ \\
15 & -0.0624909 & -0.000382022 & -0.0058225 & +0.00233719 & -0.000301143 & $+1.087 \mathrm{E}-05$ \\
\hline
\end{tabular}


Table 6.69: Fit result of Eq. (6.7) to different apertures and models, for the correction to infinite aperture, as a function of the measured size of a given cluster. NICMOS/NIC2 chip, F205W filter, post-cooler

\begin{tabular}{|c|c|c|c|c|c|c|}
\hline \multicolumn{7}{|c|}{ King 30 } \\
\hline Aperture (pixels) & $a$ & $b$ & $c$ & $d$ & $e$ & $f$ \\
\hline 2 & -2.66932 & +1.756 & -0.553406 & +0.0571251 & -0.00227409 & $+2.21232 \mathrm{E}-05$ \\
3 & -2.73101 & +1.91859 & -0.560754 & +0.0577039 & -0.00239811 & $+2.83536 \mathrm{E}-05$ \\
4 & -2.55201 & +1.85826 & -0.525915 & +0.0540785 & -0.00229576 & $+2.93695 \mathrm{E}-05$ \\
5 & -2.21234 & +1.67256 & -0.467719 & +0.0477899 & -0.00201664 & $+2.54496 \mathrm{E}-05$ \\
6 & -2.05292 & +1.56394 & -0.429664 & +0.0436856 & -0.00184185 & $+2.32928 \mathrm{E}-05$ \\
7 & -1.90083 & +1.42548 & -0.381527 & +0.0378624 & -0.00153234 & $+1.72445 \mathrm{E}-05$ \\
8 & -1.70516 & +1.24694 & -0.323042 & +0.0304258 & -0.00109859 & $+7.5906 \mathrm{E}-06$ \\
9 & -1.5104 & +1.07485 & -0.269042 & +0.0237396 & -0.000718932 & $-5.94517 \mathrm{E}-07$ \\
10 & -1.28687 & +0.884562 & -0.210334 & +0.0161962 & -0.000264795 & $-1.11254 \mathrm{E}-05$ \\
11 & -1.11278 & +0.744708 & -0.169074 & +0.0112212 & $+1.30295 \mathrm{E}-05$ & $-1.7028 \mathrm{E}-05$ \\
12 & -0.936904 & +0.607308 & -0.129212 & +0.0063907 & +0.00028662 & $-2.2967 \mathrm{E}-05$ \\
13 & -0.774395 & +0.481103 & -0.0929017 & +0.00199852 & +0.0005347 & $-2.8315 \mathrm{E}-05$ \\
14 & -0.650979 & +0.385504 & -0.0659554 & -0.00108557 & +0.00069579 & $-3.14411 \mathrm{E}-05$ \\
15 & -0.541901 & +0.29964 & -0.0416337 & -0.00389571 & +0.00084449 & $-3.43793 \mathrm{E}-05$ \\
\hline
\end{tabular}


Table 6.70: Fit result of Eq. (6.7) to different apertures and models, for the correction to infinite aperture, as a function of the measured size of a given cluster. NICMOS/NIC2 chip, F205W filter, post-cooler

\begin{tabular}{|c|c|c|c|c|c|c|}
\hline \multicolumn{7}{|c|}{ EFF 15 } \\
\hline Aperture (pixels) & $a$ & $b$ & $c$ & $d$ & $e$ & $f$ \\
\hline 2 & -1.30741 & +0.534905 & -0.211575 & +0.0196822 & -0.000644025 & $+3.19457 \mathrm{E}-06$ \\
3 & -1.00034 & +0.407642 & -0.130705 & +0.00733475 & +0.000138716 & $-1.49538 \mathrm{E}-05$ \\
4 & -0.660999 & +0.231902 & -0.0630815 & -0.00104962 & +0.000584989 & $-2.36377 \mathrm{E}-05$ \\
5 & -0.383964 & +0.117631 & -0.0301094 & -0.00374448 & +0.000642289 & $-2.26675 \mathrm{E}-05$ \\
6 & -0.293016 & +0.0894775 & -0.0205845 & -0.00375954 & +0.000560856 & $-1.90114 \mathrm{E}-05$ \\
7 & -0.245236 & +0.0616042 & -0.0101823 & -0.00417942 & +0.000524095 & $-1.68489 \mathrm{E}-05$ \\
8 & -0.203351 & +0.0340066 & -0.00169733 & -0.00443887 & +0.000488338 & $-1.49915 \mathrm{E}-05$ \\
9 & -0.17624 & +0.0192224 & +0.00220412 & -0.00419041 & +0.000430051 & $-1.28086 \mathrm{E}-05$ \\
10 & -0.161834 & +0.0184801 & +0.00106916 & -0.0033444 & +0.000340357 & $-9.99693 \mathrm{E}-06$ \\
11 & -0.14474 & +0.018839 & -0.000717105 & -0.00252877 & +0.000260966 & $-7.61526 \mathrm{E}-06$ \\
12 & -0.119533 & +0.0114595 & +0.000487301 & -0.00231656 & +0.000232316 & $-6.72812 \mathrm{E}-06$ \\
13 & -0.110903 & +0.017177 & -0.00241393 & -0.00155691 & +0.000167997 & $-4.92229 \mathrm{E}-06$ \\
14 & -0.0963679 & +0.0143246 & -0.00228577 & -0.00128895 & +0.000138728 & $-4.02812 \mathrm{E}-06$ \\
15 & -0.0942063 & +0.0181564 & -0.00358871 & -0.000924419 & +0.000108543 & $-3.21923 \mathrm{E}-06$ \\
\hline
\end{tabular}




\subsubsection{Illustrative figures}

For illustration purposes, we present a comparative plot of the different light profiles (Fig. 6.33).

In Figs. 6.34 and 6.35 we present the fitting residuals for a number of differently sized clusters and a range of fitting radii.

In Fig. 6.36 we show the differences of WFPC2 PSFs across one chip, using the WF3 chip and the F555W filter.
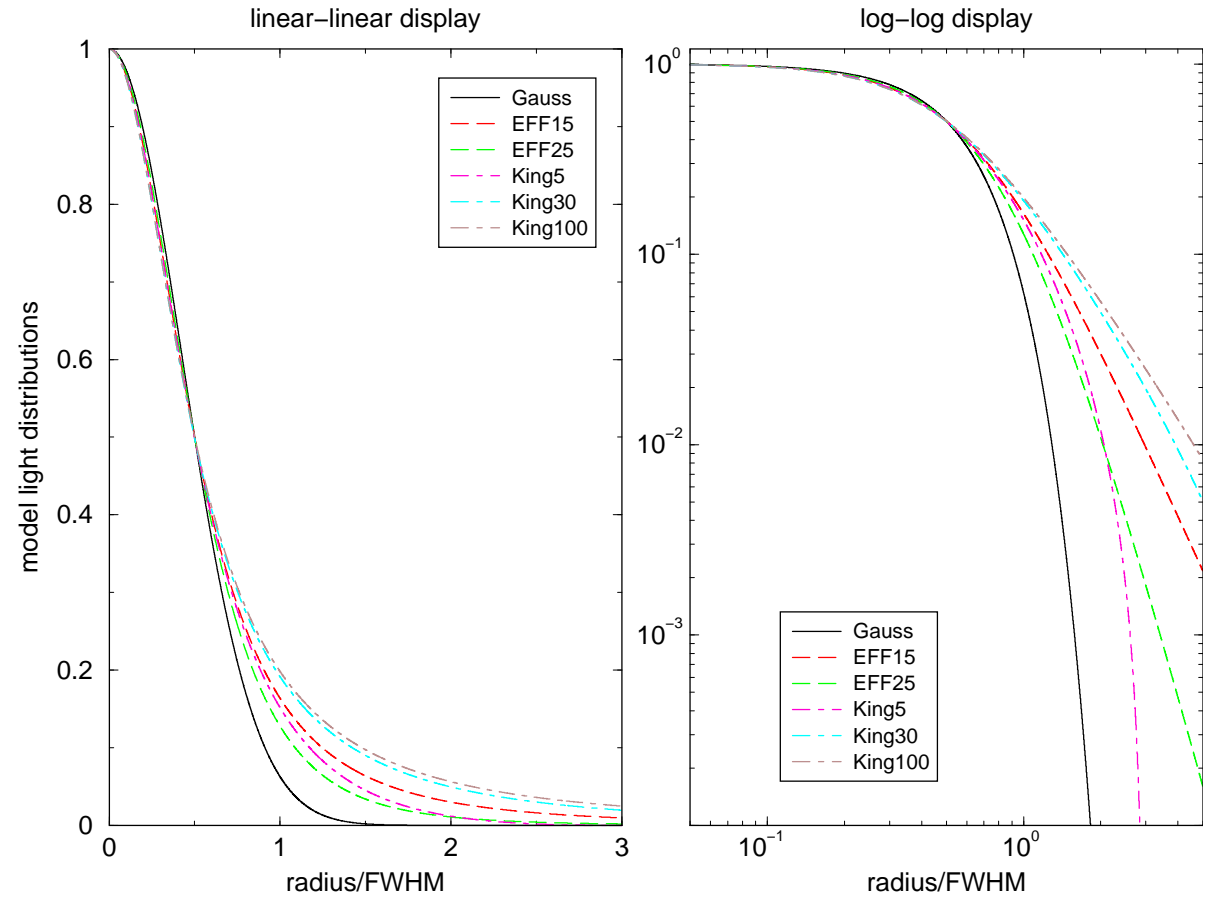

Figure 6.33: Various model light distributions. Left: Double-linear display. Right: Double-log display. 

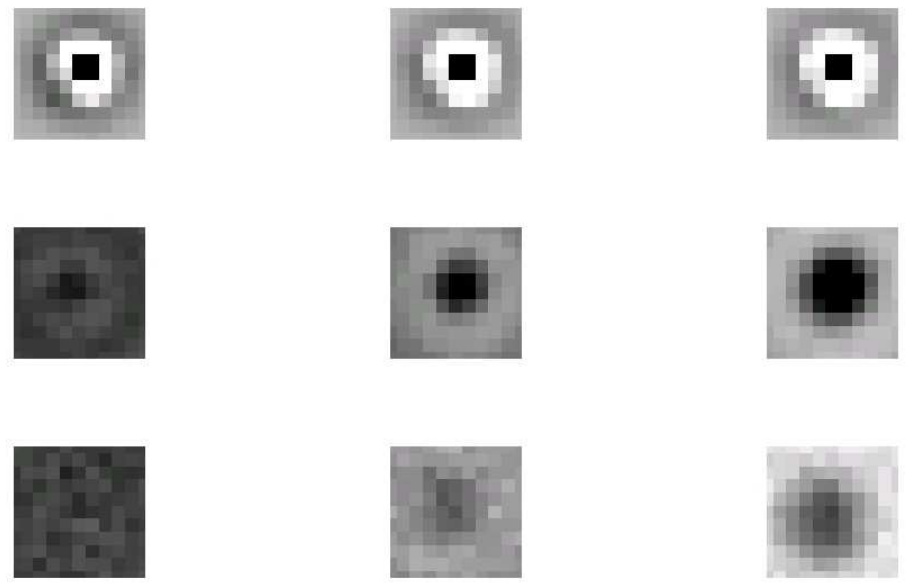

Figure 6.34: Light profile residuals of King 30 clusters. Rows (from top to bottom): $\mathrm{FWHM}=0.5,5.0$ and 10.0 pixels. Columns (from left to right): fitting radius $=5,9,15$ pixels. Color scale is linear, with very dark/bright regions having the largest deviations. Gray-scales are identical within a row.
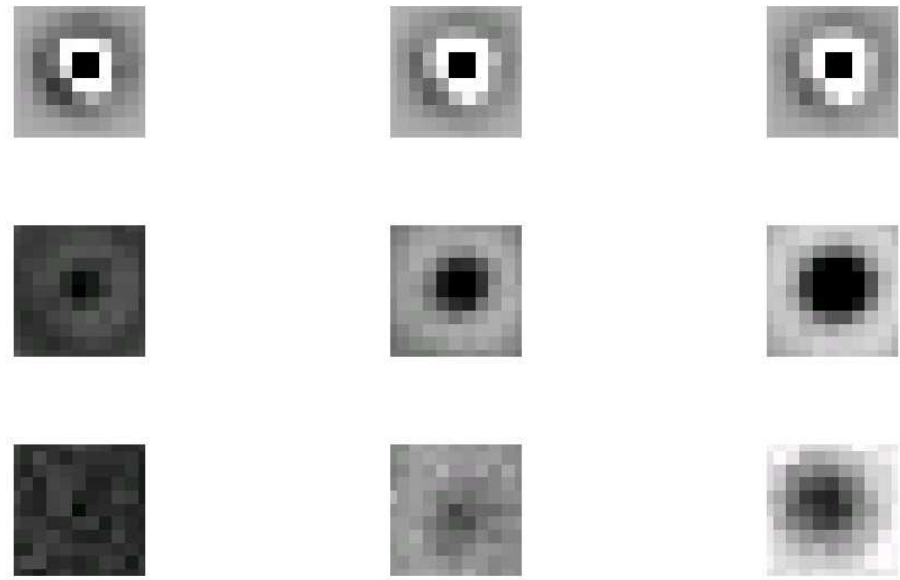

Figure 6.35: Light profile residuals of EFF 15 clusters. Rows (from top to bottom): $\mathrm{FWHM}=0.5,5.0$ and 10.0 pixels. Columns (from left to right): fitting radius $=5,9,15$ pixels. Color scale is linear, with very dark/bright regions having the largest deviations. Gray-scales are identical within a row. 

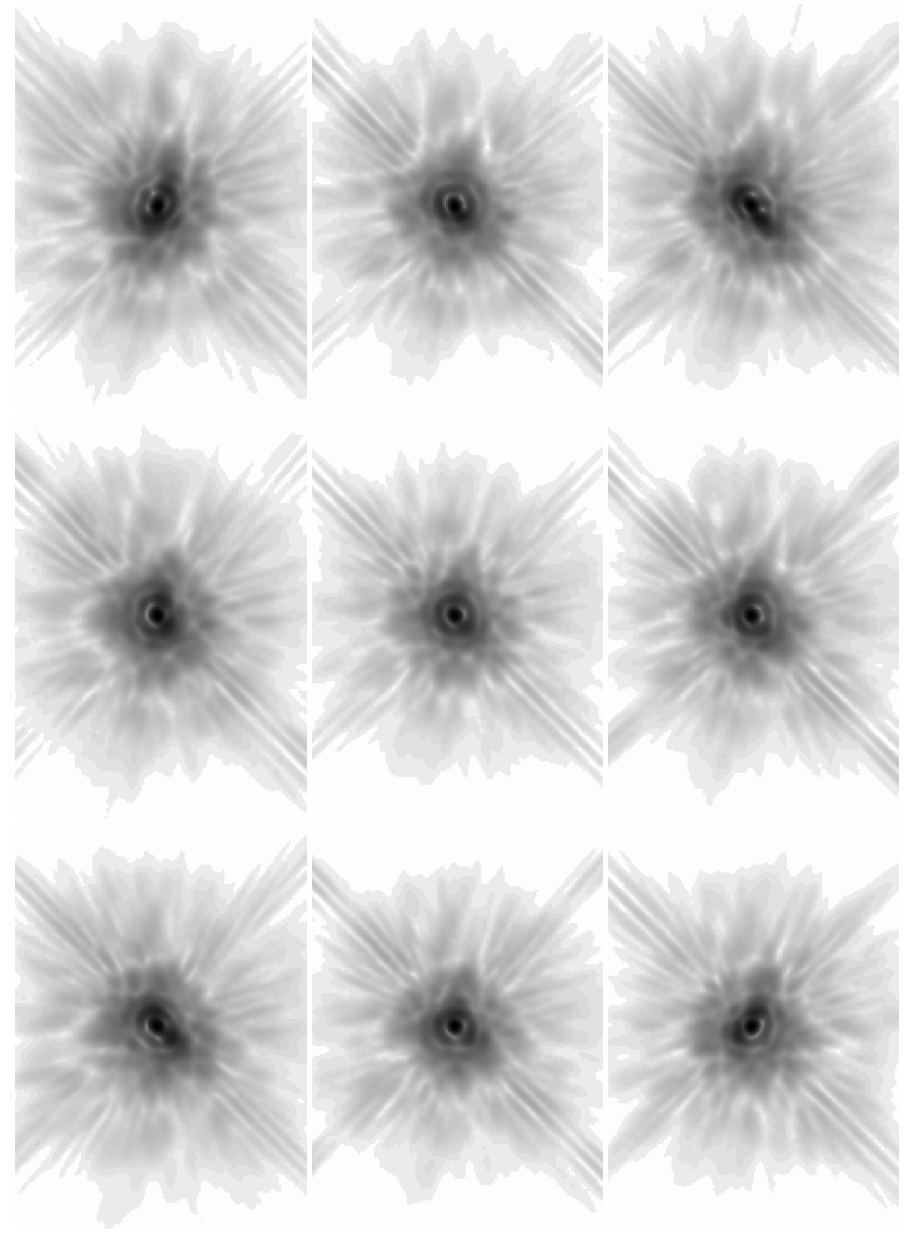

Figure 6.36: PSFs for the WF3 chip and the F555W filter, plotted using logarithmic color coding. The position in the image corresponds to the respective position on the chip. The PSFs were created subsampled by a factor of 10 , the displays show $200 \times 130$ pixels per PSF, corresponding to $2 \times 1.3$ arcsec for an observation. 



\section{Chapter 7}

\section{The Antennae star clusters: an old globular cluster system observed at its birth}

\section{$7.1 \quad$ Introduction}

Galaxies are complex systems, containing populations of stars of different ages and varying degrees of chemical enrichment, in addition to large reservoirs of gas, dust, black holes, dark matter, et cetera. Several methods can be employed to disentangle the multitude of stellar populations: (i) Integrated galaxy light always contains contributions from different populations. Its analysis requires either many ad hoc assumptions or a wealth of multi-wavelength observational data, or both. (ii) Resolved stellar population studies are possible only for the few very closest systems, where we can distinguish the individual stars, even with the high spatial resolution of the Hubble Space Telescope (HST). (iii) Star clusters (SCs), on the other hand, are very useful in this respect. They are brighter than most single stars and their integrated properties can easily be studied to much greater distances (up to $\sim 100 \mathrm{Mpc}$ ). Fortunately, crowding of SCs is usually not a serious issue, so that it is generally possible to study each cluster individually. In addition, SCs are simple systems, and modelling requires few assumptions, in contrast to that of integrated galaxy light. They are formed almost instantaneously through the collapse of a single giant molecular cloud (GMC), and hence all stars in a given SC have (almost) the same age and chemical composition.

In interacting and merging galaxies, bursts of intense star and star cluster formation are triggered, provided that sufficient gas reservoirs are available (e.g., Schweizer \& Seitzer 1998; Zepf et al. 1999; Fall \& Zhang 2001; de Grijs et al. 2003b,c). These secondary SCs form from gas pre-enriched in the merging galaxies (Fritze - v. Alvensleben 2004). SC formation is found to be an important, if not the dominant, mode of star formation in gas-rich galaxy mergers (e.g., de Grijs et al. 2003c). At least some fraction of the newly-formed SCs will survive for as long as several Gyr, and evolve into GC counterparts, as evidenced by the 1-3 Gyr-old clusters in merger remnant galaxies (de Grijs et al. 2003a, 
2005; Goudfrooij et al. 2004; Schweizer, Seitzer \& Brodie 2004). Thus, GCs represent a fossil record of the conditions in their host galaxies at the time of their formation, modified by stellar evolutionary processes which are generally well understood at present (Fritze - v. Alvensleben 2004), as well as by internal and external disruption and evaporation processes (e.g., Gnedin, Lee \& Ostriker 1999; Fall \& Zhang 2001; Vesperini 2001). The age and metallicity distributions of SC systems are therefore key clues to the evolutionary history of their parent galaxies (e.g., de Grijs et al. 2001, 2003b,c; Fritze - v. Alvensleben 2004).

\subsection{Cluster luminosity and mass functions}

The most commonly used diagnostics to explore the properties and evolution of extragalactic SC systems are their luminosity and mass functions (LFs, MFs). For old GC systems in the local Universe, these LFs and MFs are both approximately Gaussian in shape, with very similar parameters among a wide variety of galaxies (e.g., Harris 1991; Ashman \& Zepf 1998; minor trends with metal content can easily and in a straightforward manner be accounted for, cf. Ashman, Conti \& Zepf 1995). The almost universal turnover of GC LFs is often used as a "secondary" distance indicator, hence for determinations of the Hubble constant (Sandage \& Tamman 1995; Forbes 1996; Kavelaars et al. 2000) and thus the expansion rate and the age of the Universe.

Local galaxies exhibiting "normal" star formation contain young massive cluster (YMC) systems, molecular clouds and cloud cores that all feature similar power-law LFs (Zepf et al. 1999; Zhang \& Fall 2001; de Grijs et al. 2003b). For that reason, a power-law LF is usually adopted for young cluster systems in interacting galaxies as well, although the stellar contamination of such samples is more difficult to deal with, and non-negligible age differences among the YMCs may lead to distortions of the LFs with respect to their MFs (Meurer 1995; Fritze - v. Alvensleben 1999).

It has hitherto remained unclear if the difference in shape between the LFs of YMC and old GC systems is due to differences in the nature and formation of the two types of clusters, or whether the power law of young systems is secularly transformed into the Gaussian distribution of old SC systems by selective destruction effects. This fundamental question will be answered by the results presented in this letter. Classical models for the evolution of star cluster systems in galactic potentials have naturally obtained the Gaussian shape from the power-law by selectively destroying low-mass clusters (e.g., Fall \& Zhang 2001). Recent studies show that this is possible only with significant fine-tuning of the model parameters, while an initially Gaussian shape is stable under a wide range of conditions (e.g., Vesperini 2001; de Grijs, Parmentier \& Lamers 2005). The most commonly cited argument for a power-law cluster MF is its similarity to the MFs of GMCs as observed in nearby normal galaxies. However, this argument has two drawbacks: (i) It assumes that the cluster mass correlates directly with the mass of the precursor GMC. The presence of a mass-radius-relation for GMCs and its absence for clusters (Ashman \& Zepf 2001), however, casts doubt upon the validity of this assumption. (ii) It assumes that the MF of GMCs in interacting galaxies is of similar shape as in nearby normal galaxies. However, as recently shown (Gao \& Solomon 2004), of prime relevance for star formation is not the mass of the GMCs, but the mass of the dense cores embedded within 
these GMCs. The fraction of gas in these cores compared to the total gas mass is substantially higher in violently interacting and star-forming galaxies compared to "normal" star-forming isolated galaxies (Gao \& Solomon 2004). Both the MFs of GMCs and their cores will be determined only to sufficiently low masses when the next generation millimetre observatory ALMA becomes operational in the next decade.

\subsection{Observational data and analysis method}

In order to break the dead-lock in this area, and to provide the impetus for a major leap forward in our understanding of GC formation for the evolution of their host galaxies, we analysed high-resolution observational data obtained for the nearest major ongoing merger of a galaxy pair, NGC 4038/39, nicknamed the "Antennae" by virtue of their long tidal tails. At a distance of some 19 Mpc, this system can be studied in detail with the unique high spatial resolution and sensitivity of the HST. We obtained publicly available images acquired by B. Whitmore and collaborators with the Wide Field/Planetary Camera 2 (WFPC2), from the Space Telescope Data Archive operated by the Space Telescope Science Institute in Baltimore (MD), USA.

\subsubsection{Source selection and photometry}

After alignment of multiple short-exposure images we first identify sources significant at least at a $4 \sigma$ level above the sky background (where $\sigma$ is the sky noise). Applying a spatial cross-correlation technique, using the data from different passbands, we remove spurious detections, such as noise peaks and cosmic rays. Subsequently we remove bright single field stars and contaminants, such as remaining cosmic rays, from the cluster sample by carefully measuring the source sizes and accounting for the instrumental PSF. The standard way of performing photometry on star clusters is by using a set of finite concentric apertures for measuring source and sky fluxes. Ideally, these apertures would be of infinite size to contain all cluster light, but neighbouring sources and variable background set tight restrictions on the maximum aperture sizes allowed to avoid contamination. However, due to the observed size range of clusters, this method systematically underestimates the fluxes from large clusters compared to small ones. We therefore constructed artificial cluster models with a range of sizes and correct our photometry for this effect, using the measured size of the observed clusters [see Anders, Gieles \& de Grijs (2006) for details].

\subsubsection{Cluster completeness}

Subsequently, we constructed artificial clusters with parameters similar to those observed, for two representative regions in the interacting galaxy system characterised by different background source densities, and for two representative cluster sizes. Our artificial clusters spanned a range of brightnesses, allowing us to determine observational completeness fractions as a function of cluster brightness. We strongly emphasise the requirement to include all cluster selection criteria self-consistently and realistically in the construction of these completeness functions. Finally, each observed cluster was assigned the most 
appropriate completeness value for its measured parameters (brightness, size and local background density). This allowed us to correct for variations in background level and cluster brightness in a statistically meaningful manner.

\subsubsection{Statistical turnover determination}

The major difficulties associated with the detection of a Gaussian-type LF at these very young ages arise from the observational completeness and measurement uncertainties: low-luminosity objects are more likely to be missed or misclassified, particularly in regions with strongly variable background and significant crowding of sources. Unless accurately accounted for, these effects can cause an intrinsical power-law LF to show an apparent turn-over. In order to draw a significant conclusion on the existence (or absence) of a real turn-over in the data we obtained for the Antennae system, we modelled the situation as a "missing data problem," where the probability to observe a cluster is given by the completeness function at the relevant intrinsic cluster brightness. We use a Maximum Likelihood approach to determine the best-fit parameters for Gaussian and power-law LFs, respectively. Moreover, we use the ratio of these probabilities (the likelihood ratio) to compare the plausibility of the Gaussian with that of the power-law model.

\subsection{The cluster luminosity function in the An- tennae system}

Our key result is the detection of a turn-over, characteristic of a Gaussian distribution, in the cluster luminosity function at $M_{V}=-8.7 \mathrm{mag}$, where a bootstrapped $95 \%$ confidence region is given by $\approx[-9.0,-8.3] \mathrm{mag}$.

To analyse the statistical significance of our result we simulated the distribution of the likelihood ratio of the Gaussian and the power-law model assuming that the best-fit power-law model is correct. When we compare the observed likelihood ratio to the result of the simulations, it follows that the Gaussian model is more appropriate than the power-law model with a probability of error of less than $0.1 \%$. We performed the same statistical analysis using the "raw" $V$-band data (i.e., without the aperture corrections), which are by definition fainter and hence more susceptible to the impact of completeness effects (the completeness functions were determined for the fully calibrated data, including the aperture corrections). We found that a Gaussian luminosity function is preferred in this case as well. Therefore, we exclude the possibility that our result is affected by calibration errors. We performed the same statistical analysis using subsets of different ranges in cluster sizes, ages, and regions within the galaxies. We find the Gaussian distribution to be favoured for each single subset, usually with a probability of error of less than $0.1 \%$. For subsets with a small number of clusters (typically fewer than 50 clusters) the test is less stringent but still clearly favours the Gaussian distribution.

The discovery of this turn-over is of great theoretical significance, in particular because Gaussian LFs have never before been disentangled robustly at the young age of 10-100 Myr of the Antennae cluster system. Recent studies have detected similar turn-overs for intermediate-age SC systems of 1-3 Gyr (de Grijs et al. 2003a, 2005; Goudfrooij et al. 2004), but these results could 

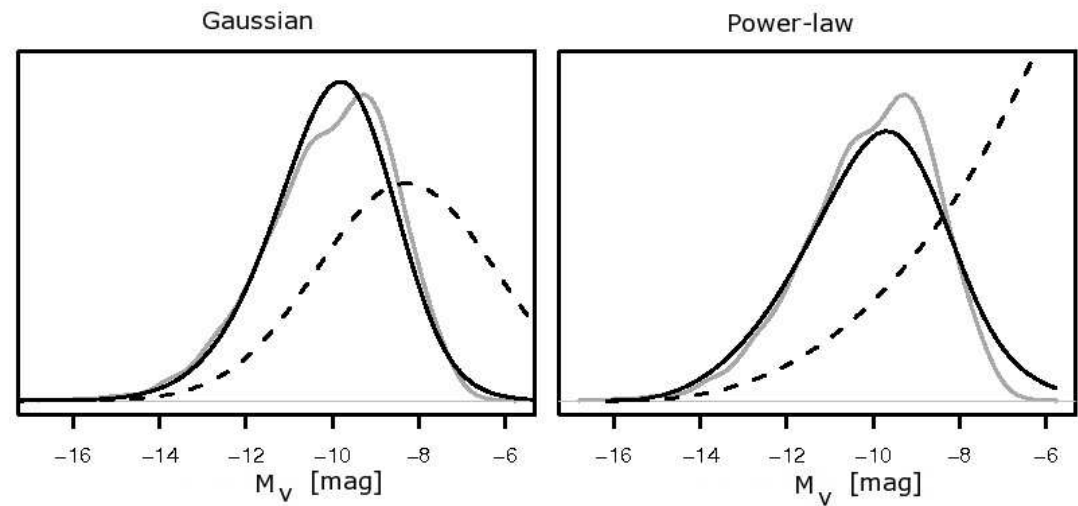

Figure 7.1: Comparing the different model fits with the observations: The best fits of the Gaussian (left panel) and the power-law (right panel) models for the absolute $V$-band LF, in arbitrary units. The grey line is a kernel density estimate of the observed data and the black line shows the Maximum Likelihood fit multiplied by the completeness function. The dashed line corresponds to the estimated LF without taking into account the varying completeness fractions (scaled arbitrarily w.r.t. the observations).

already be affected by selective star cluster disruption. In an environment as inhomogeneous as in the Antennae galaxies, cluster photometry and the determination of observational completeness is not an easy task, although of crucial importance. To the best of our knowledge, despite its obvious importance for the existence, or otherwise, of a turn-over in the young cluster LF, the approach we present here is the most detailed study to date, having taken into account the observational details (photon statistics, observational errors), completeness functions (for distinct regions and background levels in the galaxies, for different cluster sizes), and using advanced statistical methods to test the validity of our results. In Fig. 1 we show the best-fit Gaussian and power-law LFs in the $V$ band for our YMC sample. The power law yields a faint tail that is significantly overpopulated compared to the observed luminosity function (after allowing for the completeness function), whereas the predictions from the Gaussian model fit the observations overall, and particularly at the faint end $\left(M_{V}>-8 \mathrm{mag}\right)$, significantly better.

\subsection{Summary and conclusions}

Based on a careful photometric analysis of the YMC system in the nearby Antennae interacting galaxy system, we find strong evidence for the YMC system being more similar to old GC systems and less similar to YMC systems in noninteracting galaxies than claimed previously. Our result is based on a particularly careful account of the strong stellar contamination present in this ongoing starburst environment (which had probably not been completely removed from cluster samples studied previously), and on our particular care in constructing reliable completeness functions for environments as heterogeneous as for the 
clusters in the Antennae system. We deal with these difficulties by carefully measuring the source sizes, to discriminate between point sources (stars) and extended sources (clusters), by constructing completeness functions for a range of cluster parameters (cluster size, local density of background sources), and assigning the appropriate completeness corrections to each individual cluster.

Our result highlights, that the YMCs formed in nearby interacting and merging galaxies, and the associated violent starbursts, are vital diagnostics for the interpretation and understanding of galaxy formation at high redshift. Our result proves that the environment and the conditions prevalent in nearby interacting galaxies are indeed very similar to those at the time of galaxy formation in the early Universe, in the sense that they allow for the formation of rich GC systems. The apparent difference between GMC LFs in normal galaxies and the YMC LF in the Antennae galaxies can result from two testable scenarios addressed above: either from intrinsic differences already at the level of GMC core MFs in normal and interacting galaxies, or from the existence of two different modes of star-formation possibly due to differences in the ambient gas pressure (e.g., Elmegreen \& Efremov 1997). The former scenario can soon be tested by ALMA; our result would predict a turnover in the GMC core MF in violently star-forming galaxies, while past findings would imply a power-law GMC core MF for quiescent star-forming galaxies. Whatever scenario will turn out to be closest to the truth, it will revolutionise our understanding of how star formation takes place both locally and how it has proceeded since the dawn of the Universe.

\subsection{Acknowledgments}

PA, UFvA and RdG thank the International Space Science Institute (Berne, Switzerland) for research support and hospitality. PA is partially supported by DFG grant Fr 916/11-3. LB acknowledges support from the Georg Lichtenberg programme "Applied Statistics \& Empirical Methods" and the DFG graduate program 1023 "Identification in Mathematical Models". 


\section{Bibliography}

Anders P., Gieles M., de Grijs R., 2006, A\&A, in press

Ashman, K.M., Conti, A., Zepf, S.E., 1995, AJ, 110, 1164

Ashman, K.M., Zepf, S.E., 1998, Globular cluster systems (CUP: Cambridge)

Ashman, K.M., Zepf S.E., 2001, AJ, 122, 1888

de Grijs, R., O’Connell, R.W., Gallagher J.S., 2001, AJ, 121, 768

de Grijs, R., Bastian, N., Lamers, H.J.G.L.M., 2003a, ApJ, 583, L17

de Grijs, R., Anders, P., Lynds, R., Bastian, N., Lamers, H.J.G.L.M., O'Neill, E.J., Jr., 2003b, MNRAS, 343, 1285

de Grijs, R., Lee, J.T., Mora Herrera, M.C., Fritze - v. Alvensleben, U., Anders, P., 2003c, New Astron., 8, 155

de Grijs R., Parmentier G., Lamers H.J.G.L.M., 2005, MNRAS, 364, 1054

Elmegreen, B.G., Efremov, Y.N., 1997, ApJ, 480, 235

Fall, S.M., Zhang, Q., 2001, ApJ, 561, 751

Forbes, D.A., 1996, AJ, 112, 954

Fritze - v. Alvensleben, U., 1999, A\&A, 342, L25

Fritze - v. Alvensleben, U., 2004, A\&A, 414, 515

Gao, Y., Solomon, P.M., 2004, ApJ, 606, 271

Gnedin, O.Y., Lee, H.M., Ostriker, J.P., 1999, ApJ, 522, 935

Goudfrooij, P., Gilmore, D., Whitmore, B.C., Schweizer, F., 2004, ApJ, 613, L121

Harris, W.E., 1991, ARA\&A, 29, 543

Kavelaars, J.J., Harris, W.E., Hanes, D.A., Hesser, J.E., Pritchet, C.J. 2000, ApJ, 533, 125

Meurer, G.R., 1995, Nature, 375, 742

Sandage, A., Tammann, G.A., 1995, ApJ, 446, 1 
Schweizer, F., Seitzer, P., 1998, AJ, 116, 2206

Schweizer, F., Seitzer, P., Brodie, J.P., 2004, AJ, 128, 202

Vesperini, E., 2001, MNRAS, 322, 247

Zepf, S.E., Ashman, K.M., English, J., Freeman, K.C., Sharples, R.M., 1999, AJ, 118,752

Zhang, Q., Fall, S.M., Whitmore, B.C., 2001, ApJ, 561, 727 


\section{Chapter 8}

\section{Further publications}

In the course of the work constituting the main body of the present thesis, side projects have emerged and additional publications were produced. Only the abstracts (and references) of these additional publications will be presented in this chapter.

\subsection{The photometric evolution of dissolving star clusters I: First predictions}

\section{Lamers H.J.G.L.M., Anders P., de Grijs R., 2006, A\&A, submitted}

Observations and $N$-body simulations suggest that cluster-wide mass segregation occurs in the early phase of a star cluster, i.e. within about $20 \%$ of its total lifetime. We calculate the broad-band photometric evolution of unresolved star clusters, including mass segregation and the preferential loss of low-mass stars, in a simplified way. The stellar mass function of a cluster evolves due to three effects: (a) the evolution of the massive stars reduces their number; (b) tidal effects before cluster-wide mass segregation reduce the mass function homogeneously, i.e. independently of the stellar mass; (c) after mass segregation has occurred, tidal effects remove the lowest-mass stars from the cluster. These effects result in a narrowing of the stellar mass range. These three effects are described quantitatively, following the results of $N$-body simulations, and taken into account in the calculation of the photometric history, based on the GALEV cluster evolution models for solar metallicity and a Salpeter mass function. We find the following results:

(1) During the first $\sim 40 \%$ of the lifetime of a cluster its colour evolution is adequately described by the standard GALEV models (without mass segregation) but the cluster gets fainter due to the loss of stars by tidal effects. During this phase the colour evolution is the same for clusters with and without initial mass segregation.

(2) Between $\sim 40$ and $\sim 80 \%$ of its lifetime (independent of the total lifetime) the cluster gets bluer due to the loss of low-mass stars. This will result in an underestimate of the age of clusters if standard cluster evolution models are used. The correction increases from 0.15 dex for a cluster with a total lifetime of 3 Gyr to 0.5 dex for clusters with a total lifetime of 30 Gyr. 
(3) After $\sim 80 \%$ of the total lifetime of a cluster it will rapidly get redder. This is because stars at the low-mass end of the main sequence, which are preferentially lost, are bluer than the AGB stars that dominate the light at long wavelengths. This will result in an overestimate of the age of clusters if standard cluster evolution models are used.

(4) Clusters with mass segregation evolve along almost the same tracks in colourcolour diagrams as clusters without mass segregation. Therefore it will be difficult to distinguish the effect of mass segregation from that due to the cluster age. Only if the total lifetime of clusters can be estimated then the colours can be used to give reliable age estimates.

(5) The changes in the colour evolution of unresolved clusters due to mass segregation will affect the determination of the star formation histories of galaxies if they are derived from clusters that have a total lifetime of less than about 30 Gyr.

(6) The effect of mass segregation on the photometric history of clusters might explain the presence of old ( $\sim 13$ Gyr) clusters in NGC 4365 which are photometrically disguised as intermediate-age clusters $(2-5 \mathrm{Gyr})$, if the expected total lifetime of these clusters is between 16 and 32 Gyr. It may also explain the concentration of these clusters towards the center of NGC 4365.

\subsection{How well do we know the age and mass dis- tributions of the star cluster system in the Large Magellanic Cloud?}

\section{de Grijs R., Anders P., 2006, MNRAS, in press}

The Large Magellanic Cloud (LMC) star cluster system offers the unique opportunity to independently check the accuracy of age (and the corresponding mass) determinations based on a number of complementary techniques. Using our sophisticated tool for star cluster analysis based on broad-band spectral energy distributions (SEDs), "AnalySED", we reanalyse the Hunter et al. (2003) LMC cluster photometry. Our main aim is to set the tightest limits yet on the accuracy of absolute age determinations based on broad-band SEDs, and therefore on the usefulness of such an approach. Our broad-band SED fits yield reliable ages, with statistical absolute uncertainties within $\Delta \log ($ Age/yr $) \simeq$ 0.4 overall. The systematic differences we find with respect to previous age determinations are caused by conversions of the observational photometry to a different filter system, thus leading to systematically inaccurate results.

The LMC's cluster formation rate (CFR) has been roughly constant outside of the well-known age gap between $\sim 3$ and 13 Gyr, when the CFR was a factor of $\sim 5$ lower. Using a simple approach to derive the characteristic cluster disruption time-scale, we find that $\log \left(t_{4}^{\text {dis }} / \mathrm{yr}\right)=9.9 \pm 0.1$, where $t_{\text {dis }}=t_{4}^{\text {dis }}\left(M_{\mathrm{cl}} / 10^{4} \mathrm{M}_{\odot}\right)^{0.62}$. This long characteristic disruption time-scale implies that we are observing the initial cluster mass function (CMF). We conclude that while the older cluster (sub)samples show CMF slopes that are fully consistent with the $\alpha \simeq-2$ slopes generally observed in young star cluster systems, the youngest mass and luminosity-limited LMC cluster subsets show shallower slopes (at least below masses of a few $\times 10^{3} \mathrm{M}_{\odot}$ ), which is contrary to dynamical expectations. This may imply that the initial CMF slope of the LMC cluster 
system as a whole is not well represented by a power-law, although we cannot disentangle the unbound from the bound clusters at the youngest ages.

\subsection{Systematic uncertainties in the analysis of star cluster parameters based on broad-band imaging observations}

de Grijs R., Anders P., Lamers H.J.G.L.M., Bastian N., Parmentier G., Sharina M.E., Yi S., 2005, MNRAS, 359, 874

High-resolution Hubble Space Telescope (HST) imaging observations of star cluster systems provide a very interesting and useful alternative for stellar population analyses to spectroscopic studies with $8 \mathrm{~m}$-class telescopes. Here, we assess the systematic uncertainties in (young) cluster age, mass, and - to a lesser extent - extinction and metallicity determinations, based on broad-band imaging observations with the HST. Our aim here is to intercompare the results obtained using a variety of commonly used modelling techniques, specifically with respect to our own extensively tested multi-dimensional approach. Any significant differences among the resulting parameters are due to the details of the various, independently developed modelling techniques used, rather than to the stellar population models themselves. Despite the model uncertainties and the selection effects inherent to most methods used, we find that the peaks in the relative age and mass distributions of a given young $\left(\lesssim 10^{9} \mathrm{yr}\right)$ cluster system can be derived relatively robustly and consistently, to accuracies of $\sigma_{t} \equiv \Delta\langle\log ($ Age $/ \mathrm{yr})\rangle \leq 0.35$ and $\sigma_{M} \equiv \Delta\left\langle\log \left(M_{\mathrm{cl}} / \mathrm{M}_{\odot}\right)\right\rangle \leq 0.14$, respectively, assuming Gaussian distributions in cluster ages and masses for reasons of simplicity. The peaks in the relative mass distributions can be obtained with a higher degree of confidence than those in the relative age distributions, as exemplified by the smaller spread among the peak values of the mass distributions derived. This implies that mass determinations are mostly insensitive to the approach adopted. We reiterate that as extensive a wavelength coverage as possible is required to obtain robust and internally consistent age and mass estimates for the individual objects, with reasonable uncertainties. Finally, we conclude that the actual filter systems used for the observations should be used for constructing model colours, instead of using conversion equations, to achieve more accurate derivations of ages and masses.

\subsection{CIRPASS near-infrared integral-field spec- troscopy of massive star clusters in the star- burst galaxy NGC 1140}

de Grijs R., Smith L.J., Bunker A., Sharp R.G., Gallagher J.S., Anders P., Lancon A., O'Connell R.W., Parry I.R., 2004, MNRAS, 352, 263

We analyse near-infrared integral field spectroscopy of the central starburst region of NGC 1140, obtained at the Gemini-South telescope equipped with CIRPASS. Our $\sim 1.45-1.67 \mu \mathrm{m}$ wavelength coverage includes the bright $[\mathrm{Fe}$ 
II $\lambda 1.64 \mu \mathrm{m}$ emission line, as well as high-order Brackett (hydrogen) lines. While strong $[\mathrm{Fe} \mathrm{II}]$ emission, thought to originate in the thermal shocks associated with supernova remnants, is found throughout the galaxy, both $\mathrm{Br} 12-4$ and $\mathrm{Br}$ 14-4 emission, and weak $\mathrm{CO}(6,3)$ absorption, is predominantly associated with the northern starburst region. The Brackett lines originate from recombination processes occurring on smaller scales in (young) HiI regions. The time-scale associated with strong [Fe II] emission implies that most of the recent starformation activity in NGC 1140 was induced in the past $\sim 35-55$ Myr. Based on the spatial distributions of the [Fe II] versus Brackett line emission, we conclude that a galaxy-wide starburst was induced several tens of Myr ago, with more recent starburst activity concentrated around the northern starburst region.

This scenario is (provisionally) confirmed by our analysis of the spectral energy distributions of the compact, young massive star clusters (YMCs) detected in new and archival broad-band Hubble Space Telescope images. The YMC ages in NGC 1140 are all $\lesssim 20$ Myr, consistent with independently determined estimates of the galaxy's starburst age, while there appears to be an age difference between the northern and southern YMC complexes in the sense expected from our CIRPASS analysis. Our photometric mass estimates of the NGC 1140 YMCs, likely upper limits, are comparable to those of the highest-mass Galactic globular clusters and to spectroscopically confirmed masses of (compact) YMCs in other starburst galaxies. Our detection of similarly massive YMCs in NGC 1140 supports the scenario that such objects form preferentially in the extreme environments of interacting and starburst galaxies.

\subsection{Star Cluster Formation and Evolution in Nearby Starburst Galaxies: II. Initial Con- ditions}

de Grijs R., Anders P., Lynds R., Bastian N., Lamers H.J.G.L.M., O'Neill E.J., Jr., 2003, MNRAS, 343, 1285

We use the ages, masses and metallicities of the rich young star cluster systems in the nearby starburst galaxies NGC 3310 and NGC 6745 to derive their cluster formation histories and subsequent evolution. We further expand our analysis of the systematic uncertainties involved in the use of broad-band observations to derive these parameters (Paper I) by examining the effects of a priori assumptions on the individual cluster metallicities. The age (and metallicity) distributions of both the clusters in the circumnuclear ring in NGC 3310 and of those outside the ring are statistically indistinguishable, but there is a clear and significant excess of higher-mass clusters in the ring compared to the non-ring cluster sample; it is likely that the physical conditions in the starburst ring may be conducive for the formation of higher-mass star clusters, on average, than in the relatively more quiescent environment of the main galactic disc. For the NGC 6745 cluster system we derive a median age of $\sim 10$ Myr. NGC 6745 contains a significant population of high-mass "super star clusters", with masses in the range $6.5 \lesssim \log \left(M_{\mathrm{cl}} / M_{\odot}\right) \lesssim 8.0$. This detection supports the scenario that such objects form preferentially in the extreme environments of interacting galaxies. The age of the cluster populations in both NGC 3310 and NGC 
6745 is significantly lower than their respective characteristic cluster disruption time-scales, respectively $\log \left(t_{4}^{\text {dis }} / \mathrm{yr}\right)=8.05$ and 7.75 , for $10^{4} M_{\odot}$ clusters. This allows us to obtain an independent estimate of the initial cluster mass function slope, $\alpha=2.04( \pm 0.23)_{-0.43}^{+0.13}$ for NGC 3310, and $1.96( \pm 0.15) \pm 0.19$ for NGC 6745 , respectively, for masses $M_{\mathrm{cl}} \gtrsim 10^{5} M_{\odot}$ and $M_{\mathrm{cl}} \gtrsim 4 \times 10^{5} M_{\odot}$. These mass function slopes are consistent with those of other young star cluster systems in interacting and starburst galaxies.

\subsection{Star Cluster Formation and Evolution in Nearby Starburst Galaxies: I. Systematic Uncertainties}

de Grijs R., Fritze - v. Alvensleben U., Anders P., Gallagher J.S., Bastian N., Taylor V.A., Windhorst R.A., 2003, MNRAS, 342, 259

The large majority of extragalactic star cluster studies done to date have essentially used two or three-passband aperture photometry, combined with theoretical stellar population synthesis models, to obtain age, mass and extinction estimates, and sometimes also metallicities. The accuracy to which this can be done depends on the choice of (broad-band) passband combination and, crucially, also on the actual wavelengths and the wavelength range covered by the observations. Understanding the inherent systematic uncertainties (the main aim of this paper) is of the utmost importance for a well-balanced interpretation of the properties of extragalactic star cluster systems.

We simultaneously obtain ages, metallicities and extinction values for $\sim 300$ clusters in the nearby starburst galaxy NGC 3310, based on archival Hubble Space Telescope observations from the ultraviolet (UV) to the near-infrared (NIR). We show that, for ages $6 \lesssim \log ($ age $/$ yr $) \lesssim 9$, and if one can only obtain partial coverage of the spectral energy distribution (SED), an optical passband combination of at least four filters including both blue and red passbands results in the most representative age distribution, as compared to the better constrained ages obtained from the full UV-NIR SED coverage. We find that while blue-selected passband combinations lead to age distributions that are slightly biased towards younger ages due to the well-known age-metallicity degeneracy, red-dominated passband combinations should be avoided.

NGC 3310 underwent a (possibly extended) global burst of cluster formation $\sim 3 \times 10^{7}$ yr ago. This coincides closely with the last tidal interaction or merger with a low-metallicity galaxy that likely induced the formation of the large fraction of clusters with (significantly) subsolar metallicities. The logarithmic slope of the $V$-band cluster luminosity function, for clusters in the range $17.7 \lesssim \mathrm{F} 606 \mathrm{~W} \lesssim 20.2 \mathrm{mag}$, is $\alpha_{\mathrm{F} 606 \mathrm{~W}} \simeq-1.8 \pm 0.4$. The observed cluster system has a median mass of $\left\langle\log \left(m / M_{\odot}\right)\right\rangle \simeq 5.25 \pm 0.1$, obtained from scaling the appropriate model SEDs for known masses to the observed cluster SEDs. 


\subsection{Stellar Populations and Star Cluster Forma- tion in Interacting Galaxies with the Ad- vanced Camera for Surveys}

de Grijs R., Lee J.T., Mora Herrera M.C., Fritze - v. Alvensleben U., Anders P., 2003, New Astronomy, 8, 155

Pixel-by-pixel colour-magnitude and colour-colour diagrams - based on a subset of the Hubble Space Telescope Advanced Camera for Surveys Early Release Observations - provide a powerful technique to explore and deduce the star and star cluster formation histories of the MiCE and the TADPOLE interacting galaxies.

In each interacting system we find some 40 bright young star clusters $(20 \lesssim$ $\mathrm{F} 606 \mathrm{~W}(\mathrm{mag}) \lesssim 25$, with a characteristic mass of $\left.\sim 3 \times 10^{6} M_{\odot}\right)$, which are spatially coincident with blue regions of active star formation in their tidal tails and spiral arms. We estimate that the main events triggering the formation of these clusters occurred $\sim(1.5-2.0) \times 10^{8}$ yr ago. We show that star cluster formation is a major mode of star formation in galaxy interactions, with $\gtrsim 35 \%$ of the active star formation in encounters occurring in star clusters. This is the first time that young star clusters have been detected along the tidal tails in interacting galaxies.

The tidal tail of the TADPOLE system is dominated by blue star forming regions, which occupy some $60 \%$ of the total area covered by the tail and contribute $\sim 70 \%$ of the total flux in the F475W filter (decreasing to $\sim 40 \%$ in F814W). The remaining pixels in the tail have colours consistent with those of the main disk. The tidally triggered burst of star formation in THE MiCE is of similar strength in both interacting galaxies, but it has affected only relatively small, spatially coherent areas.

\subsection{Conference proceedings}

1. Analysing Multi-Color Observations of Young Star Clusters in Mergers,

P. Anders, U. Fritze - v. Alvensleben, R. de Grijs, in Proceedings of 'New Horizons in Globular Cluster Astronomy', Padova 2002, ed. by G. Piotto, G. Meylan, G. Djorgovski, M. Riello

2. Evolutionary Synthesis Modelling of Young Star Clusters in Merging Galaxies,

P. Anders, U. Fritze - v. Alvensleben, R. de Grijs, in Proceedings of 'The Evolution of Galaxies, III. From Simple Approaches to Self-Consistent Models', Kiel 2002, Astrophys. Space Sci. (Ap\&SS) 284 (2003) 937-940, ed. by G. Hensler, G. Stasińska, S. Harfst, P. Kroupa, Chr. Theis

3. New Evolutionary Synthesis Tool for Modelling Young Star Clusters in Merging Galaxies,

P. Anders, R. de Grijs, U. Fritze - v. Alvensleben, in Proceedings of 'Extragalactic Globular Cluster Systems', Garching 2002, ed. by M. KisslerPatig 
4. Multi-color observations of Young Star Clusters,

P. Anders, U. Fritze - v. Alvensleben, R. de Grijs, in Proceedings of 'IAU General Assembly, Joint Discussion 6', Sydney 2003, ed. by T. Bridges, D. Forbes

5. From Young to Old: Spectral Models for Star Cluster Systems, U. Fritze - v. Alvensleben, P. Anders, R. de Grijs, in Proceedings of 'IAU General Assembly, Joint Discussion 6', Sydney 2003, ed. by T. Bridges, D. Forbes

6. Star Clusters and ASTROVIRTEL,

P. Anders, U. Fritze - v. Alvensleben, R. de Grijs, in Proceedings of 'IAU General Assembly, Joint Discussion 8', Sydney 2003, ed. by F. Genova, Xiangqun Cui

7. Young star clusters: Progenitors of globular clusters!?,

P. Anders, U. Fritze - v. Alvensleben, R. de Grijs, in Proceedings of 'IAU General Assembly, Joint Discussion 11', Sydney 2003, included in Highlights of Astronomy, Vol. 13

8. The Initial Mass Function of Star Cluster Systems,

U. Fritze - v. Alvensleben, R. de Grijs, P. Anders, in Proceedings of 'IAU General Assembly, Joint Discussion 11', Sydney 2003, included in Highlights of Astronomy, Vol. 13

9. Young star clusters: Metallicity tracers in external galaxies, P. Anders, U. Fritze - v. Alvensleben, R. de Grijs, in Proceedings of 'IAU General Assembly, Joint Discussion 21', Sydney 2003, included in Highlights of Astronomy, Vol. 13

10. Young star clusters: Clues to galaxy formation and evolution, P. Anders, U. Fritze - v. Alvensleben, R. de Grijs, in Proceedings of 'IAU General Assembly, Symposium 217', Sydney 2003, ed. by P.-. Duc, J. Braine, E. Brinks

11. Young Star Clusters: Lighthouses in the dark,

P. Anders, U. Fritze - v. Alvensleben, R. de Grijs, in Proceedings of 'IAU General Assembly, Symposium 221', Sydney 2003, ed. by M. Burton, R. Jayawardhana, T. Bourke.

12. Mass Functions of Young Star Cluster Systems,

U. Fritze - v. Alvensleben, P. Anders, R. de Grijs, in Proceedings of 'IAU General Assembly, Symposium 221', Sydney 2003, ed. by M. Burton, R. Jayawardhana, T. Bourke.

13. Measuring sizes and compactnesses of Young Star Clusters, P. Anders, M. Gieles, R. de Grijs, U. Fritze - v. Alvensleben, in Proceedings of 'Starbursts - From 30 Doradus to Lyman break galaxies', Cambridge 2004, ed. by R. de Grijs, R. M. González Delgado, Ap\&SS 329, p.P3

14. Nearby star cluster formation: Probes of cosmological galaxy evolution, 
P. Anders, R. de Grijs, U. Fritze - v. Alvensleben, in Proceedings of 'Rencontres de Moriond, XXVth Moriond Astrophysics Meeting', La Thuile (I) 2005, ed. by D. Elbaz, H. Aussel 


\section{Chapter 9}

\section{Summary \& Outlook}

\subsection{Summary}

This thesis represents a significant step forward for systematic studies of young star cluster systems. I have studied a number of caveats and solutions associated with young star cluster analyses. I have developed and carefully tested advanced image reduction and data analysis tools for star cluster systems in general, in particular for those recently formed in major starbursts which accompany galaxy interaction/merger events.

The work can be divided into three large topics:

- providing up-to-date models applicable to the study of young star cluster systems (the new GALEV models and a "cookbook" on how to improve size measurements and aperture photometry of star clusters), my main interest here resided in broad-band photometry

- studying the uncertainties related to these models and the parameter determination of star clusters using the new models (including the statistically robust and well-tested ANALYSED tool to determine physical cluster parameters from observations)

- applying the developed methods to observations of a large variety of star cluster systems.

Our working group is constantly maintaining and updating the GALEV evolutionary synthesis code developed by U. Fritze - v. Alvensleben. I have included the effects of gaseous emission to the code, allowing the prediction of earlier evolutionary stages than previous models (starting now at $4 \mathrm{Myr}$ whereas previous models started at $140 \mathrm{Myr}$ ). These early stages are partially dominated by gaseous emission. This emission arises from the hard flux of hot young stars ionising their surrounding remnants of the parental gas clouds. We use observed average line ratios for low metallicities $\left(\mathrm{Z} \leq 0.004=1 / 5 \mathrm{Z}_{\odot}\right)$ and theoretical values for higher metallicities. Those data sets for heavy element lines are suited best for their respective metallicity ranges. The impact of both emission lines and continuum emission are now included in the GALEV code. As I have shown in Sect. 2, the effect of gaseous emission can amount up to $80 \%$ of the total flux, depending on the age and metallicity of the cluster and 
the filter used. While the gaseous emission is negligible for cluster ages older than $\sim 15$ Myr, it can completely dominate the photometry of younger clusters, significantly altering their spectral energy distributions (SEDs). Because of the application of average line ratios our models are not suitable for detailed line ratio studies, which have to make use of dedicated photoionisation codes. I have added a number of frequently used filter sets (particularly all relevant cameras on-board the HST). Models for a wide range of filter systems are now available at http://www.astro.physik.uni-goettingen.de/ galev/panders/SSPModels/

index.html.

These new models diminish the need to convert between different filter sets and therefore reduce associated errors significantly.

The study of uncertainties of evolutionary synthesis models and of cluster parameter determinations from observations has recently become a very active field of research. In collaboration with N. Bissantz I developed a statistically robust tool to compare the GALEV models with observations of star clusters reliably. This ANALYSED tool returns the best-matching physical parameters (age, metallicity, mass, extinction within the host galaxy) for each cluster observed (provided accurate photometry in suitable passbands is available, see below), and the associated $1 \sigma$ uncertainty ranges for each parameter. To do so the tool compares the observations with the full grid of GALEV models, consisting of a huge data grid ( $\sim 120.000$ models) of spectra and photometry as a function of cluster age, metallicity and internal extinction within the host galaxy of the cluster. While the ANALYSED tool was created to be used for photometric star cluster studies, it is applicable to a broader range of studies, with only minor adjustments (like analyses of spectroscopic indices of star clusters, photometric redshift determinations et cetera).

The tool was carefully tested on artifical clusters (see Sect. 3). For this purpose, I took cluster SEDs from our models with well-defined parameters and added Gaussian noise to simulate observational uncertainties. Hereupon, I applied the ANALYSED tool to redetermine the cluster parameters, and compared input parameters with redetermined parameters. Using input cluster models spanning the entire parameter space of our models, I was able to identify several aspects relevant for planning and analysing star cluster studies:

1. In order to constrain cluster parameters efficiently, a wavelength coverage as long as possible is essential. Ideally, this includes UV as well as NIR passbands. Whereas the inclusion of UV passbands significantly improves the age determination (and partially the metallicity determination), NIR passbands help constrain the metallicity. For ages $\leq$ few Gyr, the most important standard filters are the $\mathrm{U}$ and $\mathrm{B}$ band. For ages $\geq$ few Gyr, the $\mathrm{B}$ and $\mathrm{V}$ band (and to a lesser extent the $\mathrm{U}$ band) are most important instead. Clearly, the more passbands are available, the better the cluster parameters can be constrained (for similar wavelength coverage).

2. With increasing observational uncertainties there are clear trends for the redetermined parameters: Except for the youngest input ages (below a few 10s Myr), ages, metallicities and masses are systematically underestimated, and counterbalanced by overestimates of the internal extinction values (age-extinction degeneracy). For the youngest input ages slight trends seem to overestimate ages, extinction and masses while underes- 
timating the metallicity (age-metallicity degeneracy). In addition, with increasing observational uncertainties the overall parameter uncertainties increase.

3. Apart from the oldest test clusters (with input ages of $10 \mathrm{Gyr}$ ), the parameter determination accuracies are fairly insensitive to the input values for metallicity and internal extinction. For the oldest test clusters trends to underestimate age (and correspondingly to overestimate extinction; age-extinction degeneracy) more strongly with decreasing input extinction/increasing input metallicity are seen. Deviations are stronger for more limited spectral coverage.

4. A priori assumptions and restrictions of the analysis (e.g. to fix the metallicity to solar metallicity as often done in the literature) should be handled with extreme care. If these assumptions are not met by the clusters (or at least the vast majority of the clusters in a sample), the results are highly unpredictable.

5. The tool is robust in the sense that the redetermined parameters converge to the input values for decreasing observational errors, increasing the spectral coverage (both in terms of number of filters and the covered wavelength range), or by partially restricting the analysis to the input values.

Whereas the exact values are only valid for our models and the ANALYSED tool, the general trends are valid for all comparable methods. This work suggests certain improvements for observing strategies and allows for a considerably improved subsequent data analysis.

Similar tests, though covering a smaller part of the parameter space, have been performed by R. de Grijs, collaborators and myself using the observed cluster photometry of the young star cluster systems of the nearby starburst ring galaxy NGC 3310 and the starburst galaxy NGC 6745 (see Sect. 8.5 and 8.6). The main results of our artificial cluster studies on the wavelength coverage/choice of filters and a priori assumptions for the metallicity are fully confirmed by this observational study. Due to the limited parameter space occupied by the clusters in NGC 3310, the other aspects accessible to the artificial cluster tests could not be evaluated.

Further studies about the reliability of cluster parameter determinations from comparison of observations with evolutionary synthesis models were performed in collaboration with R. de Grijs.

The "cluster fitting challenge" (see Sect. 8.3) aimed at quantifying the differences between the methods used by various groups. We provided all the participating groups with the same data sets and inter-compared the various results. We find that, despite significant scatter, peaks within the parameter distributions (namely for ages and masses) of young ( $\leq 1 \mathrm{Gyr}$ ) star clusters can be derived relatively robustly and consistently. We can quantify the relative accuracies of $\sigma_{t} \equiv \Delta\langle\log ($ Age $/ \mathrm{yr})\rangle \leq 0.35$ and $\sigma_{M} \equiv \Delta\left\langle\log \left(M_{\mathrm{cl}} / \mathrm{M}_{\odot}\right)\right\rangle \leq 0.14$, respectively, assuming Gaussian distributions in cluster ages and masses for reasons of simplicity.

In addition, we compared results obtained with the ANALYSED tool (utilising integrated broad-band imaging) of a sample of LMC star clusters with 
results independently determined from isochrone fitting of these star clusters' resolved stellar populations (i.e. their CMDs), see Sect. 8.2. We were able to estimate the absolute accuracy of parameters derived from integrated broad-band imaging with respect to the (presumably more accurate) method of CMD fitting. We found the absolute statistical uncertainties to be within $\Delta \log$ (Age/yr) $\simeq 0.4$ overall. Both above-mentioned accuracies have to be kept in mind for any given analysis of star cluster systems.

With regards to observations, my main interest resided in studying the young star cluster system in the nearby dwarf starburst galaxy NGC 1569 . By analysing archival multi-colour HST observations, I was able to increase the cluster sample in this galaxy roughly by a factor 4 with respect to previous studies. The main conclusions are as follows:

1. I confirm the bursty character of star cluster formation already previously found. The most recent burst started roughly $25 \mathrm{Myr}$ ago and seems to decline currently although sites of active star formation are observed. The low number of clusters older than roughly $25 \mathrm{Myr}$ prevents the accurate determination of previous bursts. However, older clusters seem to have formed on a significantly lower, roughly constant level. Our ages of the two well-studied young "super" star clusters A \& B are consistent with spectroscopic age estimates available from the literature.

2. On average, the cluster masses are well below values of Galactic globular clusters, but are more consistent with Galactic open clusters, typically of the order of $10^{4} M_{\odot}$. Only four clusters have masses in excess of 2 $\times 10^{5} M_{\odot}$, the average mass of Galactic globular clusters. Concerning the two young "super" star clusters A \& B, spectroscopic masses are within a factor of $2-3$ consistent with our values, which is within the uncertainties of, e.g., the stellar initial mass function used.

3. I detect a significant lack of high-mass clusters formed in the end of the present burst of cluster formation as compared to the clusters formed in the beginning of the burst. I propose the heating of the interstellar medium by the hard radiation field of hot young stars and the energy input from supernovae of the first generation of star clusters formed in the recent burst to be of prime relevance. However, the final solution for this issue is still to be found.

The ANALYSED tool and the new GALEV models have been extensively applied and tested in the interpretation of further data, see Sect. 8.2-8.7.

After finalising the NGC 1569 star cluster work, discussions led to new impulses on how to improve the photometry of extended sources. Due to diffraction effects and charge diffusion on CCDs, even point sources (e.g. stars) appear extended. This artificially extended image of a point source is called "Point Spread Function", or PSF, of the instrument. In principle, this PSF is infinitely extended, although the majority of the flux originates from the few innermost pixels. The observed size of intrinsically extended objects (such as nearby star clusters or galaxies) is therefore a convolution of its intrinsic size with the PSF of the instrument used. The best spatial resolution is achieved with space-based observatories, especially with the HST. Star clusters within a distance $\lesssim 20 \mathrm{Mpc}$ 
appear extended in HST observations. While this allows to determine the sizes of these objects, commonly applied photometric methods are insufficient to deal with extended objects: Both PSF fitting and finite aperture photometry systematically underestimate the flux of an extended cluster with respect to a point source.

For aperture photometry the flux of a source is measured in a concentric aperture, and the local sky background is estimated with an additional outer annulus. For a point source the flux fraction outside the chosen source aperture can be estimated by applying so-called "aperture corrections". For extended sources, these aperture corrections become a function of the source size. However, our work represents the first systematic size-dependent determination of aperture corrections. Only with reliable cluster sizes the cluster photometry and the physical parameters of the clusters can be derived accurately.

I utilised the image analysis tool BAOLAB by S. Larsen to obtain artificial cluster images which reproduce observations most realistically. With these artificial clusters I studied how size determination and aperture corrections can be achieved most reliably as a function of the cluster size. To improve the general applicability, we chose a Gaussian to fit the light profiles with. This approach introduces some non-linearities, as the intrinsic cluster light profiles deviate from a Gaussian. These non-linearities are fully taken into account in the derived results.

I provide a "cookbook" for observers containing fitted equations to:

- transform measured sizes into intrinsic sizes

- calculate aperture corrections as a function of intrinsic size or measured size

- estimate which fraction of cluster light was erroneously accounted for as sky background.

These equations are presented for:

1. different cluster light profiles

2. cameras, camera chips and filters on-board the HST

3. different methods of size fitting (whether or not taking the PSF into account while fitting)

In addition, I investigated the impact of different cluster positions on the respective camera chips, subpixel shifts of cluster position, total cluster magnitude, and different levels of sky noise. I found no significant deviations from the standard setting.

However, I found a strong dependence of the results on the fitting radius, which determines the cluster area taken into account in the fit procedure. This dependence is partially explained by the non-linearities originating from the choice of fitting profile (the Gaussian).

Finally, I compared my results to the widely used DeltaMag method. With the DeltaMag method, the size of a cluster is estimated from the magnitude difference in 2 different source apertures (usually radii of 0.5 and 3 pixel are chosen). For our results I find an accuracy improvement of a factor of at least 
3-10 compared to the DeltaMag method. In addition, I identify several caveats inherent to the DeltaMag method, as this method is not robust against subpixel shifts of the cluster.

The first application of the new size determination and aperture corrections method is the young star cluster system of the nearest major galaxy merger, the "Antennae" galaxies (NGC 4038/39).

I have put great effort into objective and reproducible source selection, photometry and observational completeness determination. The completeness functions were determined for different cluster sizes and different regions in the galaxies (characterised by different sky background levels). Furthermore, in collaboration with N. Bissantz und L. Boysen we developed an statistically advanced tool to test the shape of the cluster luminosity function. This tool takes into account the observational completeness function as well as the distribution of observational uncertainties. It independently determines the probability for a Gaussian and for a power-law, and compares these probabilities (a "likelihood-ratio test"). The parameters of the distributions are determined by a maximum-likelihood method, while the parameter uncertainties are calculated from boot-strapping. In a second step, the significance of this likelihood ratio is quantified with Monte-Carlo simulations. At a 99.9\% level, I find the Gaussian distribution to fit better than the power-law, in contrast to previous claims. This result is confirmed at a statistically significant level for almost each studied subset of clusters. This project is currently finalised and prepared for publication.

To conclude:

- I have developed the most up-to-date evolutionary synthesis models, in particular for the studies of star clusters. These new models allow to study younger stellar populations than before, and due to the extended range of photometric systems provided (and therefore the diminished need to convert between different systems) allow for more accurate physical parameter determinations thasn previously possible.

- I have developed the ANALYSED tool, and extensively tested it on artificial and observed star clusters.

- I obtained a wealth of new and exciting results on a number of star cluster systems in starbursting and interacting galaxies, i.e. their physical parameters with unprecedented accuracy.

- The results have been published (or are presently submitted) in 12 refereed research articles and a number of conference proceedings. The new models and algorithms are applied by numerous groups worldwide.

\subsection{Outlook}

At present, I'm working on further improving the GALEV models. The effects of dynamical cluster evolution, mass segregation (the dynamical redistribution of stars: high-mass stars sink towards the cluster centre, while low-mass stars are redistributed to the cluster outskirts) and interaction with the surrounding galaxy potential lead to preferential removal of low-mass stars from the cluster. 
This preferential mass loss leads to changes in the cluster photometry and the derived age estimates of clusters (the changes in age can be as large as a factor of ten), as we show in a recently submitted paper. This paper's abstract is presented in Sect. 8.1.

In addition, I am studying further aspects of uncertainties inherent to evolutionary synthesis modelling and parameter determination. These projects are still in progress. 



\section{Chapter 10}

\section{Acknowledgements}

First of all, I would like to thank my thesis advisors apl. Prof. U. Fritze - v. Alvensleben und Dr. R. de Grijs. Without their constant support, teaching and discussions this thesis would not have been possible.

In addition, I would like to thank a number of persons for many fruitful exchanges of ideas and a good time. While the total list would be too long, I would like to single out some special people. This includes the Galaxy Evolution Group at the University of Göttingen (namely Thomas Lilly, Jens Bicker, Thorsten Tepper García, Peter Weilbacher) and other present of former members of the Institute of Astrophysics at the University of Göttingen, like Polichronis Papaderos, Steffen Knollmann and Matthias Zetzl. Many thanks also to Henny Lamers, Remco Scheepmaker and Marcel Haas from the University of Utrecht, and Soeren Larsen (soon Utrecht as well) for constant help. Sweet remembrance to the late Myon (IoA Cambridge, UK).

Privately I am deepest in debt to Annika, my beloved fiancée. My parents have been a constant well of reassurance and guidance. Some of the best moments of my leisure time in the course of my PhD work I have spent with the folks of the gorgeuos Göttingen students theatre ThOP. All the best for future projects!

This work is partially supported by DFG grant Fr 916/11-1, Fr 916/11-2, and Fr 916/11-3. I also acknowledge partial funding from the Marie Curie Fellowship programme EARASTARGAL "The Evolution of Stars and Galaxies", funded by the European Commission under 5FP contract HPMT-CT-2000-00132 for my extended research visit to Cambridge (UK). Thanks to the International Space Science Institute in Berne (Switzerland) for their hospitality and research support, as part of an International Team programme.

This work is based on archival observations with the NASA/ESA Hubble Space Telescope, obtained at the Space Telescope Science Institute, which is operated by the Association of Universities for Research in Astronomy (AURA), Inc., under NASA contract NAS 5-26555. It is also partially based on ASTROVIRTEL research support, a project funded by the European Commission under 5FP Contract HPRI-CT-1999-00081. This research has made use of NASA's Astrophysics Data System Abstract Service. This research has also made use of the NASA/IPAC Extragalactic Database (NED) which is operated by the Jet Propulsion Laboratory, California Institute of Technology, under contract with the National Aeronautics and Space Administration. 



\title{
Chapter 11
}

\section{Curriculum Vitae}

\author{
Family Name Anders \\ First Name Peter \\ Date of Birth $\quad 1^{\text {st }}$ September 1976 \\ Place of Birth Berlin, Germany \\ Citizenship German
}

\section{Education}

- PhD project

- from January 2002 Universität Göttingen (Germany)

- intended date of PhD defence: $20^{\text {th }}$ February 2006

- title: Formation and evolution of star clusters in interacting galaxies

- supervisors: apl. Prof. Dr. U. Fritze - v. Alvensleben (Göttingen, Germany) \& Dr. R. de Grijs (Sheffield, UK)

- Master (MPhil) project

- October 2000 - September 2001 University of Loughborough (UK)

- subject: physics (MPhil)

- title: Investigation of the shape - memory properties in the ferromagnetic $\mathrm{Ni}_{2} \mathrm{MnGa}$ system

- supervisor: Prof. K. Ziebeck

- Diploma studies: physics

- October 1996 - September 2000 Humboldt - Universität zu Berlin (Germany)

- September 1998: intermediate examination, grade point average $(\mathrm{GPA})=1.3$ (out of $5,1.0$ being best)

- 1999 - 2000: 3 (out of 4) final examinations, $\mathrm{GPA}=1.6$ (out of $5,1.0$ being best)

- Schooling

- September 1983 - September 1996, Berlin (Germany)

- school leaving examination (Abitur) in June 1996, F. S. Archenhold - Gymnasium Berlin - Treptow

- major courses: mathematics / physics item GPA: 1.4 (out of 6, 1.0 being best) 



\section{Lebenslauf}

$\begin{array}{ll}\text { Nachname: } & \text { Anders } \\ \text { Vorname: } & \text { Peter } \\ \text { Geburtstag: } & 1^{\text {st }} \text { September } 1976 \\ \text { Geburtsort: } & \text { Berlin } \\ \text { Staatsangehörigkeit: } & \text { Deutsch }\end{array}$

\section{Ausbildung}

\section{- Promotion}

- seit Januar 2002 an der Universität Göttingen (Deutschland)

- angestrebtes Datum für Verteidigung: 20. Februar 2006

- Titel: "Formation and evolution of star clusters in interacting galaxies"

- Betreuer: apl. Prof. Dr. U. Fritze - v. Alvensleben (Göttingen, Deutschland) \& Dr. R. de Grijs (Sheffield, Großbritannien)

\section{- Masterarbeit}

- Oktober 2000 - September 2001 University of Loughborough (Großbritannien)

- Studiengang: Physik (MPhil)

- Titel: Investigation of the shape - memory properties in the ferromagnetic $\mathrm{Ni}_{2} \mathrm{MnGa}$ system

- Betreuer: Prof. K. Ziebeck

\section{- Diplomstudien: Physik}

- Oktober 1996 - September 2000 Humboldt - Universität zu Berlin (Deutschland)

- September 1998: Vordiplom, Notendurchschnitt $=1.3$

- 1999 - 2000: 3 (von 4) Diplomprüfungen, Notendurchschnitt = 1.6

\section{- Schulische Ausbildung}

- September 1983 - September 1996, Berlin (Deutschland)

- Abitur im Juni 1996, am F. S. Archenhold - Gymnasium Berlin Treptow

- Leistungskurse: Mathematik / Physik

- Notendurchschnitt $=1.4$ 
